\title{
Laser induced fluorescence studies of ion acceleration in single and multiple species expanding plasmas
}

loana A. Biloiu

West Virginia University

Follow this and additional works at: https://researchrepository.wvu.edu/etd

\section{Recommended Citation}

Biloiu, loana A., "Laser induced fluorescence studies of ion acceleration in single and multiple species expanding plasmas" (2009). Graduate Theses, Dissertations, and Problem Reports. 2832.

https://researchrepository.wvu.edu/etd/2832

This Dissertation is protected by copyright and/or related rights. It has been brought to you by the The Research Repository @ WVU with permission from the rights-holder(s). You are free to use this Dissertation in any way that is permitted by the copyright and related rights legislation that applies to your use. For other uses you must obtain permission from the rights-holder(s) directly, unless additional rights are indicated by a Creative Commons license in the record and/ or on the work itself. This Dissertation has been accepted for inclusion in WVU Graduate Theses, Dissertations, and Problem Reports collection by an authorized administrator of The Research Repository @ WVU.

For more information, please contact researchrepository@mail.wvu.edu. 


\title{
Laser Induced Fluorescence Studies of Ion Acceleration in Single and Multiple Species Expanding Plasmas
}

\author{
Ioana A. Biloiu \\ Dissertation Submitted to the College of Arts and Sciences at West \\ Virginia University in partial fulfillment of the requirements of the degree \\ of \\ Doctor of Philosophy \\ In \\ Plasma Physics
}
Earl Scime, PhD., Committee Chair Arthur Weldon, PhD. Jack Littleton, PhD. Fred King, PhD. Ray Raylman, PhD.

Department of Physics, Morgantown, West Virginia 2009

Keywords: Multi-component Helicon Plasma, Electric Double Layer, Electron Energy Distribution Function, Ion Velocity Distribution Function, Laser Induced Fluorescence Tomography 


\title{
Abstract \\ Laser Induced Fluorescence Studies of Ion Acceleration in Single and Multiple Species Expanding Plasmas
}

\author{
Ioana A. Biloiu
}

Ion acceleration in single (Ar) and two positive ion species plasmas (Ar-Xe and $\mathrm{He}-\mathrm{Ar}$ ) has been investigated through laser induced fluorescence (LIF) measurements of the ion velocity distribution function (ivdf). The investigations focused on the expansion region of a helicon plasma source where, for certain operating conditions (pressure in the source less than 2 mTorr, rf driving frequency of $9.5 \mathrm{MHz}$, source axial magnetic field strength of $700 \mathrm{G}$, and expansion chamber magnetic field strength between 0 and $70 \mathrm{G}$ ), an electric double layer (EDL) was observed to form. In pure argon plasma, $4 \mathrm{~cm}$ upstream from the helicon source-expansion chamber junction, the $\mathrm{Ar}^{+}$ivdf is bimodal comprised of a slow, nearly stationary ion population $(\sim 2.3 \mathrm{~km} / \mathrm{s})$ and a fast, supersonic ion population $\left(\sim 6 \mathrm{~km} / \mathrm{s} \cong 1.8 c_{\mathrm{Ar}+}\right)$ created by the acceleration through the EDL. As the divergence of the field is increased, $19 \mathrm{~cm}$ deeper in the expansion region the fast ion group peak velocity determined by oblique incidence LIF is shifted toward higher speeds $\left(\sim 10.5 \mathrm{~km} / \mathrm{s} \cong 2.9 c_{\mathrm{Ar}+}\right)$, indicative of a second ion acceleration mechanism presence. In $\mathrm{He}-\mathrm{Ar}$ plasma the $\mathrm{Ar}^{+}$ivdf is also bimodal. As the helium fraction increases from 0 to $30 \%$, the axial flow speed of the fast ion group increases from 5.3 to $7.8 \mathrm{~km} / \mathrm{s}$. Both the fast and slow argon ion group population densities (proportional to LIF amplitude), decrease as the helium fraction increases. The slow population almost completely disappears at a helium fraction of $\sim 30 \%$. Similar effects were observed for Ar-Xe plasmas in which the lighter ion was argon. Although no $\mathrm{Xe}^{+}$beam was observed, addition of argon led to an increase in the speed of the background ion population from $1.3 \mathrm{~km} / \mathrm{s}$ in pure xenon to $2.2 \mathrm{~km} / \mathrm{s}$ for an $87.5 \% \mathrm{Ar} /(\mathrm{Ar}+\mathrm{Xe})$ ratio. In pulsed argon plasma, time resolved LIF measurements showed a time lag in the appearance of the fast ion population. The time lag was found to be a function of the pulse repetition frequency and duty cycle. Two-dimensional LIF provided additional insights into the origins of the accelerated ion population: the nearly isotropic slow population is a locally created background population whereas the distorted velocity distribution of the fast population is consistent with an origin upstream of the measurement location. 


\section{Acknowledgements}

Foremost, I would like to thank my advisor, Professor Earl Scime, for providing me both academic and financial support during my graduate research at WVU. I have learned a lot from him and I am thankful for his patience, encouragement, and guidance during these five years. I would also like to express my appreciation and thanks to all my committee members, Professor Arthur Weldon, Professor Jack Littleton, Professor Fred King, and Professor Ray Raylman. In particular I would like to thank Dr. Xuan Sun, Dr. Amy Keesee, and Dr. Robert Hardin for their useful discussions and thoughts. Thanks to my colleague, Saikat Thakur for his assistance and support during my long spending nights in the lab. My thanks extend to our secretaries, Siobhan Byrne and Sherry Puskar for their administrative and personal help. Finally, I cannot forget the ex-undergrads, Ryan Murphy and Zane Harvey, who did a lot of work, and might even have learned something in the process.

My family deserves the most special thanks of all. Thanks to my lovely daughter, Irina, who understood that "mom is busy" and endured my lack of time. Last, but not least, I am grateful to my husband, Costel, who encouraged me during the completion of this research. 


\section{Table of Contents:}

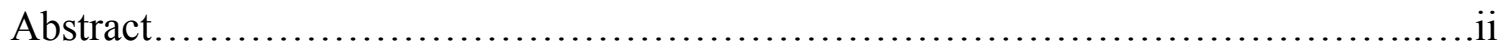

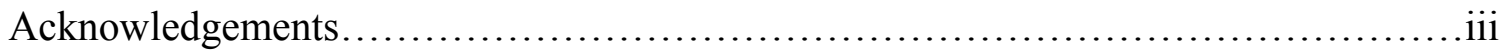

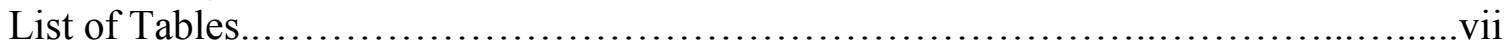

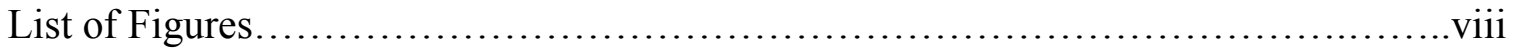

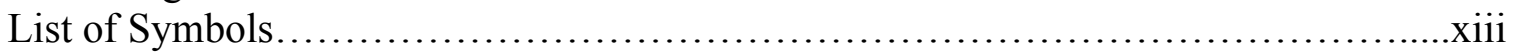

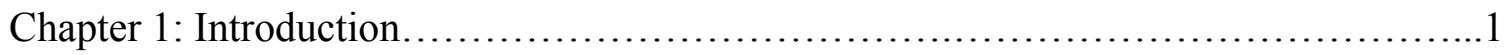

Chapter 1 References...........................................................

Chapter 2: Plasma Sheath and Double Layers.......................................10

2.1. Plasma-Wall Interaction............................................. 10

2.2. The Bohm criterion for Single Ion Species Plasma........................11

2.3. The Bohm criterion for Two-Positive Ion Species Plasma......................14

2.4. Sheath and Electric Double Layer Analogies and Differences.................16

2.5. Theoretical Description of a Plasma EDL: the Sagdeev Potential............19

2.6. Electric Double Layers in Multi-Component Plasma...........................23

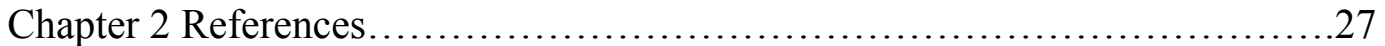

Chapter 3: The Physics of Helicon Plasma Sources.....................................29

3.1. Introduction to Helicon Plasma Sources...................................29

3.2. Operation Principles of Helicon Plasma Sources..............................30

3.3. WVU Helicon Plasma Source...............................................32

3.3.1. Helicon Source and the Diffusion Chamber........................32

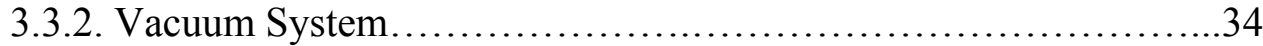

3.3.3. The Magnetic Field System.......................................35

3.3.4. Rf Generator, Matching Network, and Antenna.....................37

3.3.5. Typical Plasma Parameters in HELIX-LEIA.........................37

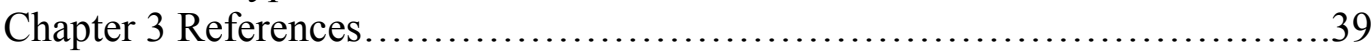

Chapter 4: Plasma Diagnostics....................................................40

4.1. Langmuir Probe ...................................................... 40

4.1.1. Druyvesteyn Differentiation Method for Obtaining Electron Energy Distribution Function (EEDF)....................46

4.1.2. Electron Energy Distribution Function from Planar Langmuir Probe Measurements..................................47

4.1.3. Electron Energy Distribution Function from Cylindrical Langmuir Probe Measurements ...............................48

4.2. Optical Emission Spectroscopy ......................................53

4.2.1. Collisional Processes in Plasma: The Corona Model................53 
4.2.1.a. Electron Impact Excitation..............................55

4.2.1.b. The Role of Metastables....................................55

4.2.1.c. Electron Impact Ionization.............................56

4.2.1.d. Heavy Particle Impact Ionization........................56

4.2.2. Determination of Relative Ion Fractions from Optical

Emission Spectroscopy (OES) ................................57

4.2.3. The OES Detection System....................................60

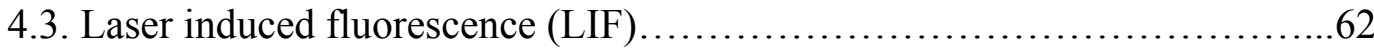

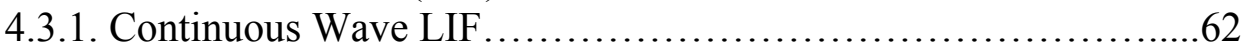

4.3.1.a. The LIF Diagnostic...................................64

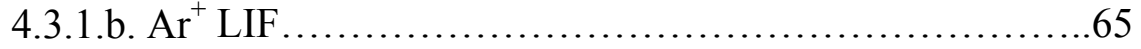

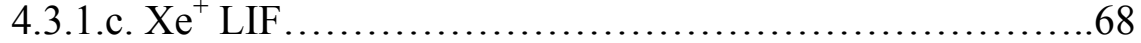

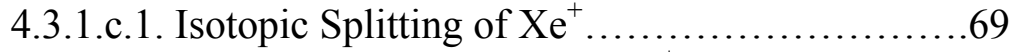

4.3.1.c.2. Nuclear-Spin Splitting of $\mathrm{Xe}^{+} \ldots \ldots \ldots \ldots \ldots \ldots . . . . . .70$

4.3.2. Time Resolved LIF.............................................76

4.3.3. LIF at Oblique Incidence.......................................79

4.3.4. LIF Tomography ............................................ 81

4.4. Scanning Internal Probe for LIF Tomography ............................ 83

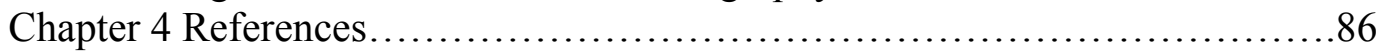

Chapter 5: Electron and Ion Energy Distribution Functions in Two-Ion Species, Helicon Plasma ......................................................... 88

5.1. Effects of Gas Composition on Plasma Density and Electron Temperature in an Ar-Xe Helicon Plasma..........................88

5.1.1. Electron Energy Distribution Function (EEDF) in the Plasma Source (HELIX).................................... 88

5.1.2. Electron Energy Distribution Function (EEDF) in the Expansion Chamber (LEIA) ...............................90

5.2. Neutral and Ion densities in Ar-Xe Plasma from OES Observations..........93

5.3. Ion Velocity Distribution Function in Single Ion Species Plasma............101

5.4. Ion Velocity Distribution Functions in Two-Ion Species Plasma............108

5.4.1. Argon IVDF in Ar-Xe Mixture.................................109

5.4.1.a. Effect of Ar/Xe Ratio on the Argon IVDF...............109

5.4.1.b. Effect of $\mathrm{Ar} / \mathrm{Xe}$ Ratio on Argon IVDF in the Expansion Region...................................121

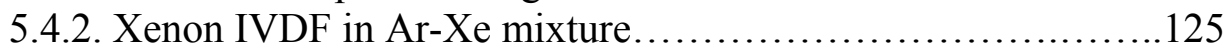

5.4.3. Argon IVDF in Ar-He mixture.................................. 130

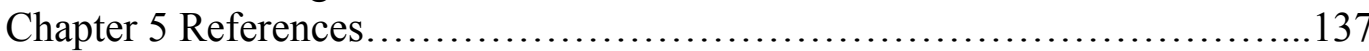

Chapter 6: Temporal Evolution of Parallel IVDF in Pulsed Argon Plasma..............139

6.1. Time-Resolved Laser Induced Fluorescence.................................139

6.2. Temporal Evolution of Bimodal Argon IVDF in

Expanding Helicon Plasma..........................................................................

Chapter 6 References................................................... 148 
Chapter 7: Oblique and Tomographic LIF Measurements of the EDL in Argon Plasma...............................................149

7.1. LIF Measurements at Oblique Incidence.................................149

7.2. Experimental Difficulties Associated with LIF Tomography ...............155

7.3. 2D Ion Velocity Distribution Functions..................................156

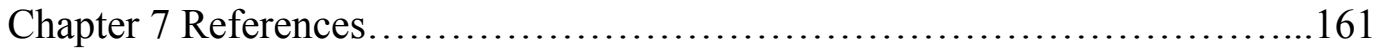

Chapter 8: Summary and Conclusions............................................ 162

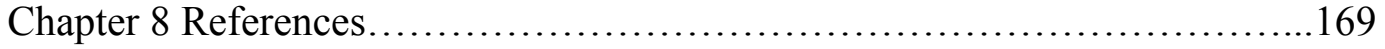

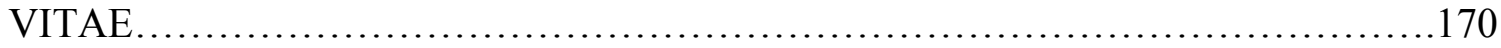




\section{List of Tables}

\section{$\underline{\text { Table }}$}

3.1. Typical plasma parameters in HELIX and LEIA............................... 38

5.1. Electron impact excitation rate coefficients for excitation of neutral argon and xenon from ground and metastable states $\left(k_{A r 0, *}^{811}, k_{X e 0, *}^{823}\right)$ and for $\mathrm{Ar}^{+}$and $\mathrm{Xe}^{+}$

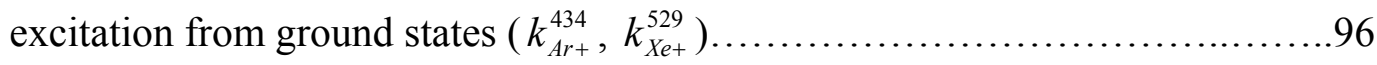

5.2. Wavelengths, transition probabilities, upper level lifetimes, and branching ratios of the investigated neutral and ionic argon and xenon emission lines.............97 


\section{List of Figures}

\section{Figure}

2.1. Schematic of the planar sheaths that build-up between plasma and grounded, conducting walls......................................................... 11

2.2. Schematic of an electric double layer (EDL) formed between two plasmas with different plasma potentials.......................................... 17

2.3. Potential distribution $\phi(x)$, ion and electron phase space distributions across an EDL with potential drop $\phi_{0}$ and internal electric field E......................18

2.4. The Sagdeev potential for a single ion species plasma double layer.............21

2.5. One dimensional profiles of a weak electric double layer potential, space charge density, and electric field...........................................23

2.6. $\quad \mathrm{Ar}^{+}$relative density $\eta$ versus $\mathrm{Ar}^{+}$and $\mathrm{He}^{+}$Mach numbers.....................26

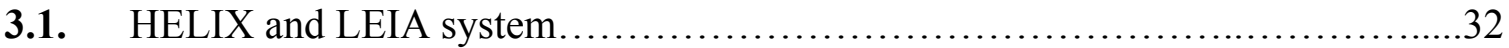

3.2. The HELIX helicon plasma source....................................... 33

3.3. HELIX-LEIA schematic with diagnostic locations A, B, C, and D labeled.......34

3.4. HELIX-LEIA system; magnetic field shape and strength, and magnetic field gradient versus axial position over the entire length of HELIX-LEIA system; spatial representation of the magnetic flux lines............................. 36

3.5. Schematic of $m=+1$ helical antenna used in these experiments................37

4.1. An idealized Langmuir I-V curve ................................... 41

4.2. I-V curve typical of a magnetized, $\operatorname{rf}$ plasma............................ 42

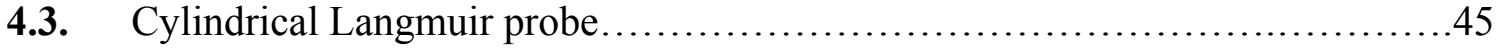

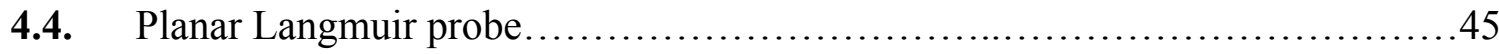

4.5. Typical planar probe $\mathrm{I}-\mathrm{V}$ characteristic and its first derivative..........................48

4.6. First and second derivative of a typical cylindrical Langmuir probe I-V characteristic

4.7. Electron energy probability function (eepf) for a quasi-Maxwellian distribution. 
4.8. Maxwell and Druyvesteyn electron energy distributions for the same average electron energy of $10 \mathrm{eV}$.

4.9. Typical Ar neutral line after background removal and spectral sensitivity correction.........................................................60

4.10. Spectral irradiance of the tungsten Oriel lamp and the measured response spectrum.

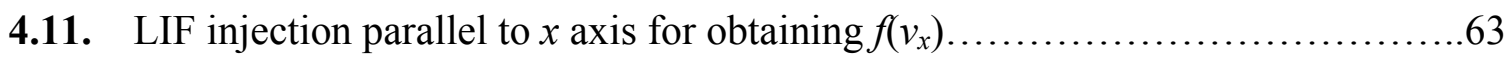

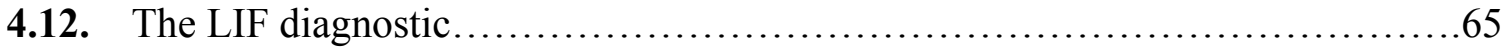

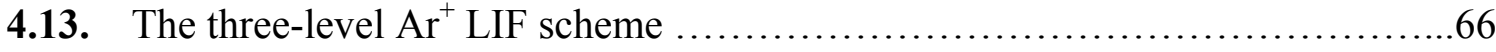

4.14. Schematic of the $\pi$ and $\sigma^{ \pm}$transitions for $\lambda_{0}=611.661 \mathrm{~nm} \mathrm{Ar}^{+}$line.............67

4.15. Evidence of Zeeman splitting in argon ion LIF measurements in HELIX for unpolarized laser injection along the magnetic field axis.....................68

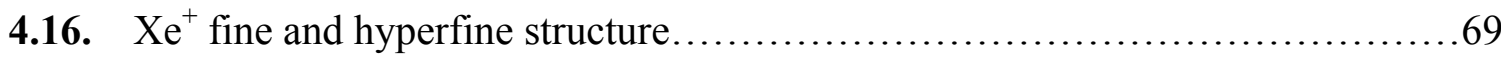

4.17. $\mathrm{Xe}^{+}$isotopic abundance and shift relative to ${ }^{132} \mathrm{Xe}^{+}$for the $5 \mathrm{~d}{ }^{4} \mathrm{D}_{7 / 2} \rightarrow 6 \mathrm{p}{ }^{4} \mathrm{P}_{5 / 2}$

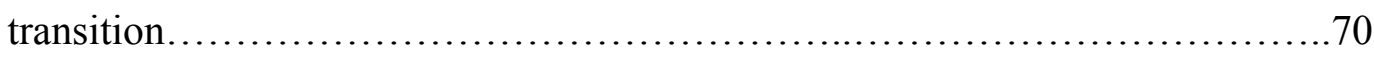

4.18. $\mathrm{Xe}^{+} 5 \mathrm{~d}^{4} \mathrm{D}_{7 / 2} \rightarrow 6 \mathrm{p}{ }^{4} \mathrm{P}_{5 / 2}$ hyperfine line splitting........................... 72

4.19. ${ }^{129} \mathrm{Xe}^{+} 5 \mathrm{~d}^{4} \mathrm{D}_{7 / 2}\left(\mathrm{~F}^{\prime}=3\right) \rightarrow 6 \mathrm{p}^{4} \mathrm{P}_{5 / 2}\left(\mathrm{~F}^{\prime \prime}=2\right)$ Zeeman line splitting.................73

4.20. ${ }^{129} \mathrm{Xe}^{+}$and ${ }^{131} \mathrm{Xe}^{+}$Zeeman shifts for $\mathrm{F}^{\prime}=3 \rightarrow \mathrm{F}^{\prime \prime}=2$ hyperfine transition $\ldots . . . . .75$

4.21. Experimental set-up for time resolved $\mathrm{Ar}^{+}$LIF diagnostic ..................77

4.22. The relative intensity of the AOM transmitted light in the 1st diffraction order versus Bragg angle misalignment; LIF signal amplitude versus injected laser

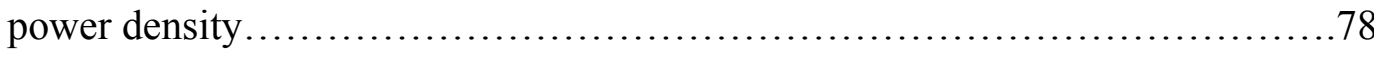

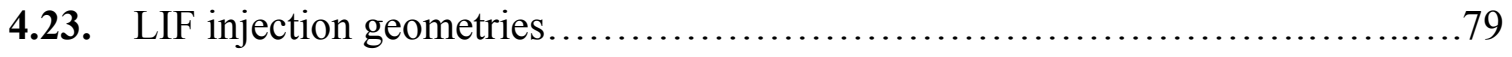

4.24. Horizontal cross-section of the HELIX-LEIA helicon source-diffusion chamber system and an expanded view of the injection geometry....................83

4.25. Scanning probe head diagnostic complement...........................85

5.1. Effective electron temperature and electron density obtained from experimentally obtained eepfs at $r=0$ in HELIX, $20 \mathrm{~cm}$ downstream from the antenna, as a function of $\mathrm{Ar} / \mathrm{Xe}$ composition. 89

5.2. A family of eedfs in Ar-Xe helicon plasma measured on the expansion chamber axis, $20 \mathrm{~cm}$ downstream the HELIX-LEIA junction. .90 
5.3. The bounding cases for the family of eedfs shown in Figure $5.2 \ldots \ldots \ldots \ldots \ldots \ldots . . .91$

5.4. Electron temperature and electron density on the axis of LEIA, $20 \mathrm{~cm}$ downstream of the HELIX-LEIA junction versus xenon fraction................92

5.5. Partial energy level diagrams for argon and xenon neutrals and the transitions used for OES investigations .94

5.6. Observed emission line intensities from argon and xenon neutral lines in HELIX at $z=126 \mathrm{~cm}$ versus xenon mixing ratio; Neutral species densities in the plasma as computed from the argon and xenon line intensities ratios.

5.7. Ion to atomic emission line intensity ratio versus xenon fraction of the total mass flow rate; the inferred $\mathrm{Ar}^{+}$and $\mathrm{Xe}^{+}$densities based on a Corona model without metastable contribution and with metastable contributions .99

5.8. OES derived total ion densities based on a Corona model with no metastable contribution and Corona model including metastable contribution; and Langmuir probe measured electron density .101

5.9. Argon ion parallel ivdf showing slow and fast ion populations in the source at $z=$ $146 \mathrm{~cm}$; the ivdf in the expansion region at $z=169 \mathrm{~cm}$

5.10. Bimodal argon ivdfs obtained in LEIA at the location $z=169 \mathrm{~cm}$ for constant magnetic field strength in HELIX of $600 \mathrm{G}$ and magnetic fields in LEIA of: $\mathrm{B}=50$ $\mathrm{G} ; \mathrm{B}=30 \mathrm{G} ; \mathrm{B}=10 \mathrm{G}$

5.11. Corrected argon ion parallel flow speeds of the slow ion population and fast ion population as a function of LEIA magnetic field

5.12. Dependence of the slow and fast ion populations LIF amplitudes and the quantity $n_{e}{ }^{2} T_{e}{ }^{1 / 2}$ on the HELIX-LEIA magnetic field ratio; Dependence of the parallel kinetic energy at the location $z=146 \mathrm{~cm}$ and $z=169 \mathrm{~cm}$ versus HELIX-LEIA magnetic field ratio

5.13. The iodine spectrum obtained from two partially overlapping $20 \mathrm{GHz}$ width laser scans and the iodine spectrum according to Salami (Ref. [31]) in the spectral range of interest for $\mathrm{Ar}^{+} \mathrm{LIF}$

5.14. The iodine spectrum obtained from three $15 \mathrm{GHz}$ partially overlapping laser scans and the iodine spectrum according to Salami (Ref. [31]) in the spectral range of interest for $\mathrm{Xe}^{+} \mathrm{LIF}$. 
5.15. LIF profile of the $\mathrm{Ar}^{+} 611.66 \mathrm{~nm}$ line in HELIX at $z=126 \mathrm{~cm} \ldots \ldots \ldots \ldots \ldots . \ldots 113$

5.16. LIF profile of the $\mathrm{Xe}^{+} 605.28 \mathrm{~nm}$ line in HELIX at $z=126 \mathrm{~cm} \ldots \ldots \ldots \ldots \ldots \ldots . . . \ldots 114$

5.17. Computed ion densities in HELIX at $z=126 \mathrm{~cm}$ as a function of xenon fraction................................................................ 115

5.18. $\mathrm{Ar}^{+}$LIF intensity and $n_{e} n_{A r^{+}} T_{e}^{1 / 2}$ product; $\mathrm{Xe}^{+}$LIF intensity and $n_{e} n_{X e^{+}} T_{e}^{1 / 2}$ product versus xenon fraction in HELIX at $z=126 \mathrm{~cm}$

5.19. Individual ion sound speeds and the system sound speed as function of xenon

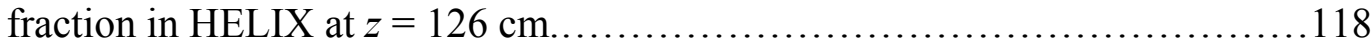

5.20. Parallel ion flow speeds in HELIX at $z=126 \mathrm{~cm}$ versus xenon fraction.

5.21. At $z=146 \mathrm{~cm}$ in HELIX, the $\mathrm{Ar}^{+}$LIF amplitude and parallel flow speed as function of small changes in the xenon fraction.

5.22. Fast $\mathrm{Ar}^{+}$population parallel velocity as function of xenon fraction at three locations close to HELIX-LEIA junction.

5.23. $\mathrm{Ar}^{+}$LIF amplitude and parallel flow speed as function of xenon fraction at $z=169$ $\mathrm{cm}$ in LEIA.

5.24. Measured floating potential; measured and calculated plasma potential $20 \mathrm{~cm}$ downstream of the HELIX-LEIA junction versus xenon fraction. .124

5.25. $\mathrm{Xe}^{+}$LIF amplitude and $\mathrm{Xe}^{+}$parallel flow speed at $z=126 \mathrm{~cm}$ and $z=146 \mathrm{~cm}$ in HELIX versus argon fraction.

5.26. $\mathrm{Xe}^{+}$and $\mathrm{Ar}^{+}$axial flow speeds in Ar-Xe plasma at $z=126 \mathrm{~cm}$ from LIF data as a function of xenon fraction; the ratio of experimentally determined parallel flow speeds and the square root of mass ratio.

5.27. $\mathrm{Xe}^{+}$parallel flow speed in HELIX and the estimated $\mathrm{Xe}^{+}$density from the LIF signal amplitude versus input rf power.

5.28. Total neutral pressure and the electron temperature in HELIX plasmas a function of helium fraction; electron density inferred from Langmuir probe measurements versus helium fraction in HELIX.

5.29. Effect of increasing helium fraction on the $\mathrm{Ar}^{+}$parallel ivdf in HELIX 132

5.30. Dependence of the metastable $\mathrm{Ar}^{+}$population obtained from integration of the ivdfs and normalized to the pure Ar case and ratio of slow/fast LIF $\mathrm{Ar}^{+}$signals versus helium fraction; parallel velocity of the fast ion population. 
5.31. Examples of $\mathrm{Ar}^{+}$bimodal parallel ivdfs for a $0 \%$ helium fraction and for a $30 \%$ helium fraction

6.1. $\mathrm{Ar}^{+}$LIF signal amplitude versus chopping frequency for three different integration times and versus integration time for a $100 \mathrm{kHz}$ chopping frequency......

6.2. Raw LIF signal during the $100 \mathrm{~ms}$ discharge pulse; Oscillations of the LIF signal amplitude observed on the first $26 \mathrm{~ms}$ of the pulse and corresponding power spectrum showing $1 \mathrm{kHz}$ oscillation frequency; LIF signal after low pass filtering; Argon ion vdf at $t=50 \mathrm{~ms}$ into the pulse.

6.3. Contour plot of the evolution of the argon ion vdf during the $100 \mathrm{~ms}$ pulse with $100 \mu$ s time resolution; Evolution of the LIF signal amplitude and ion flow velocity during the pulse

6.4. With $1 \mathrm{~ms}$ time resolution, the LIF-determined argon ion velocity distribution function during a $100 \mathrm{~ms}$ plasma pulse.....................................145

6.5. The evolution of argon ivdf during a $160 \mathrm{~ms}$ plasma pulse....................146

6.6. The evolution of argon ivdf during a $50 \mathrm{~ms}$ plasma pulse......................147

7.1. $1 \mathrm{D}$ argon ivdfs measured in the vertical plane $(\alpha, x)$ versus injection angle $\beta$.

7.2. 1D LIF obtained in the "active" probe mode; 1D LIF obtained in the "passive" probe mode 152

7.3. Examples of poor tomographic inversions................................. 155

7.4. 2D bimodal ivdf obtained $19 \mathrm{~cm}$ downstream the HELIX-LEIA junction showing the fast and slow ion populations.

7.5. Expanded views of sections of tomographic image for the slow and fast ivdfs 159

7.6. Tomographic images of the fast ion population $19 \mathrm{~cm}$ and $28 \mathrm{~cm}$ downstream from HELIX-LEIA junction for identical plasma operating conditions. 


\section{List of symbols}

\begin{tabular}{|c|c|c|}
\hline$A_{p}$ & Probe area & {$\left[\mathrm{mm}^{2}\right]$} \\
\hline$A_{S}$ & Sheath area & {$\left[\mathrm{mm}^{2}\right]$} \\
\hline$B$ & Magnetic field induction & {$[\mathrm{G}]$} \\
\hline$c_{s}$ & Ion sound speed & {$[\mathrm{m} / \mathrm{s}]$} \\
\hline$d$ & EDL thickness & {$[\mathrm{cm}]$} \\
\hline$e$ & Elementary charge & {$[\mathrm{C}]$} \\
\hline E & Electric field & {$[\mathrm{V} / \mathrm{cm}]$} \\
\hline$F$ & Total angular momentum quantum number & {$[-]$} \\
\hline$f$ & Rf driving frequency & {$[\mathrm{MHz}]$} \\
\hline$f(\varepsilon)$ & Electron energy distribution function (eedf) & {$\left[\mathrm{eV}^{-1} \mathrm{~cm}^{-3}\right]$} \\
\hline$F(\varepsilon)$ & Electron energy probability function (eepf) & {$\left[\mathrm{eV}^{-3 / 2} \mathrm{~cm}^{-3}\right]$} \\
\hline$g$ & Landé factor & {$[-]$} \\
\hline$I$ & Nuclear-spin quantum number & {$[-]$} \\
\hline$I_{i, s a t}$ & Ion saturation current & {$[\mathrm{mA}]$} \\
\hline$k$ & Wave number & {$\left[\mathrm{m}^{-1}\right]$} \\
\hline$k_{B}$ & Boltzmann constant & {$[\mathrm{J} / \mathrm{K}]$} \\
\hline$k_{L}$ & Laser propagation direction & {$[-]$} \\
\hline$k_{j}^{i z}$ & Ionization rate coefficient of species $j$ & {$\left[\mathrm{~cm}^{3} \mathrm{~s}^{-1}\right]$} \\
\hline$k_{t}^{i z}$ & Total ionization rate coefficient & {$\left[\mathrm{cm}^{3} \mathrm{~s}^{-1}\right]$} \\
\hline$L$ & Geometrical length of the plasma & {$[\mathrm{cm}]$} \\
\hline$m$ & Electron mass & {$[\mathrm{kg}]$} \\
\hline$M$ & Ion mass & {$[\mathrm{kg}]$} \\
\hline$n_{e}$ & Electron density & {$\left[\mathrm{cm}^{-3}\right]$} \\
\hline$n_{i}$ & Ion density & {$\left[\mathrm{cm}^{-3}\right]$} \\
\hline$n_{s}$ & Electron density at the sheaths edge & {$\left[\mathrm{cm}^{-3}\right]$} \\
\hline$T_{e}$ & Electron temperature & {$[\mathrm{eV}]$} \\
\hline
\end{tabular}




\begin{tabular}{|c|c|c|}
\hline$T_{\text {eff }}$ & Effective electron temperature & {$[\mathrm{eV}]$} \\
\hline$T_{i}$ & Ion temperature & {$[\mathrm{eV}]$} \\
\hline$u_{i}$ & Ion speed & {$[\mathrm{m} / \mathrm{s}]$} \\
\hline$v_{p}$ & Helicon wave's phase velocity & {$[\mathrm{m} / \mathrm{s}]$} \\
\hline$\left\langle v_{z}\right\rangle$ & Average velocity in $z$ direction & {$[\mathrm{m} / \mathrm{s}]$} \\
\hline$V_{p}$ & Plasma potential & {$[\mathrm{V}]$} \\
\hline$V_{f}$ & Floating potential & {$[\mathrm{V}]$} \\
\hline$\alpha$ & Laser injection angle & [degrees] \\
\hline$\gamma$ & Specific heat ratio & {$[-]$} \\
\hline$\Gamma_{e}$ & Electron flux & {$\left[\mathrm{cm}^{-2} \mathrm{~s}^{-1}\right]$} \\
\hline$\Gamma_{i}$ & Ion flux & {$\left[\mathrm{cm}^{-2} \mathrm{~s}^{-1}\right]$} \\
\hline$\varepsilon_{0}$ & Dielectric permittivity of the vacuum & {$\left[\mathrm{Fm}^{-1}\right]$} \\
\hline$\varepsilon$ & Electron energy & {$[\mathrm{eV}]$} \\
\hline$\langle\varepsilon\rangle$ & Mean electron energy & {$[\mathrm{eV}]$} \\
\hline$\varepsilon_{j}^{i z}$ & Ionization threshold energy of species $j$ & {$[\mathrm{eV}]$} \\
\hline$\lambda_{D}$ & Debye length & {$[\mathrm{cm}]$} \\
\hline M & Mach number & {$[-]$} \\
\hline$\Phi$ & Potential drop across the sheath & {$[\mathrm{V}]$} \\
\hline$\phi_{D L}$ & Potential drop across the EDL & {$[\mathrm{V}]$} \\
\hline$\omega_{L H}$ & Lower hybrid frequency & {$[\mathrm{MHz}]$} \\
\hline$\omega_{c e}$ & Electron cyclotron frequency & {$[\mathrm{MHz}]$} \\
\hline$\omega_{c i}$ & Ion cyclotron frequency & {$[\mathrm{MHz}]$} \\
\hline$\omega_{p e}$ & Electron plasma frequency & {$[\mathrm{MHz}]$} \\
\hline$\omega_{p i}$ & Ion plasma frequency & {$[\mathrm{MHz}]$} \\
\hline$\mu_{0}$ & Magnetic permeability in vacuum & {$\left[\mathrm{Hm}^{-1}\right]$} \\
\hline$\mu_{B}$ & Bohr magneton & {$\left[\mathrm{JT}^{-1}\right]$} \\
\hline$\sigma_{j}^{i z}$ & Ionization cross section of species $j$ & {$\left[\mathrm{~cm}^{2}\right]$} \\
\hline
\end{tabular}




\section{Chapter 1: Introduction}

A recently discovered phenomenon - spontaneous formation of a current-free electric double layer (EDL) at the open end of a low pressure helicon plasma source (in a region of divergent magnetic field), has garnered considerable interest in the plasma physics community. ${ }^{1,2}$ Since the EDL is oriented with the high potential side toward the helicon source, ions are accelerated and then ejected out of the helicon source into the expansion chamber. Observations of ion speeds larger than a few times the ion sound speed (argon and xenon ions exit speeds between 6 and $10 \mathrm{~km} / \mathrm{s}$ ) ${ }^{3,4}$ make this phenomenon of interest for ion thrusters and plasma processing applications.

The signature of EDL formation is the downstream presence of a bimodal, parallel, ion velocity distribution function (ivdf) comprised of a fast and a slow ion population. Present understanding is that these two populations have different origins: the slow ions are a background population created in the expansion region and the fast ions are accelerated by the EDL from the source into the diffusion chamber. Although measurements of energetic ions have been reported since 1990s, in the expansion region just downstream of an electron cyclotron resonance (ECR) plasma source, ${ }^{5}$ many fundamental aspects of ion acceleration in expanding magnetized plasma remain unclear. Uncertainty in the mechanism responsible for the ion acceleration is not surprising, given that at least three phenomena may operate simultaneously and separating their effects is problematic. $^{6}$

In simple geometric plasma expansion, Manheimer and Fernsler ${ }^{7}$ demonstrated theoretically that ions are accelerated without the aid of an external magnetic field or additional acceleration electrodes. In their one-dimensional isentropic flow model, the Mach number $\mathrm{M}$ is related to the variation of the cross section $S$ of the plasma flow tube

$$
\frac{d \mathrm{M}}{\mathrm{M}}=\frac{2+(\gamma-1) \mathrm{M}^{2}}{2\left(\mathrm{M}^{2}-1\right)} \frac{d S}{S}
$$

where $\gamma$ is the specific heat ratio and by definition $M$ is equal to the plasma flow speed normalized to the ion sound speed. For adiabatically steady flows $(\gamma=1)$, the singularity at the sonic point $(\mathrm{M}=1)$ was removed by including collisionality in Eq. (1.1): 


$$
\frac{d \mathrm{M}}{d z}=\frac{1}{\mathrm{M}^{2}-1}\left(\frac{\mathrm{M} d S}{S d z}-\frac{\mathrm{M}^{2} v_{i}}{c_{s}}\right)
$$

where $v_{i}$ is the ionization rate and $c_{s}=\sqrt{k_{B} T_{e} / M}$ is the ion sound speed $\left(k_{B}, T_{e}\right.$, and $M$ are the Boltzmann constant, electron temperature, and ion mass, respectively). Thus, the singularity at the sonic point is resolved not by charge separation, as in the case of electrostatic sheaths, but rather by a zero in the numerator at the same spatial position as the zero in the denominator, i.e., at the sonic point. The ion acceleration results from axial potential gradient generated by the electron pressure due to expansion. According to the model, the ion acceleration increases as the gas pressure decreases and the ion exit speed can reach up to three times the ion sound speed. Note that Eq. (1.2) does not include the effects of ambipolar or other quasi-static electric fields which, as we shall show in this work, play a critical role in ion acceleration during plasma expansion. Recently, by using three different helicon source tube diameters, Corr et al. ${ }^{8}$ demonstrated experimentally that ions can be accelerated to supersonic speed by geometric expansion alone. They showed that ion acceleration occurs for an operating pressure below a threshold value and that the ion energy scales with the ratio of the tube cross-section area to the expansion chamber cross section area.

For the case of magnetic expansion, Nakano et al. ${ }^{9}$ showed that the downstream ivdf structure is profoundly affected by the magnetic field configuration. For a mirror configuration, the parallel ivdf in the downstream diffusion region had a bimodal structure with a slow component formed by local ionization and a fast component drifting from upstream region. Part of the fast component parallel kinetic energy might arise from conversion of the upstream perpendicular kinetic energy to parallel flow energy through magnetic moment conservation. Changing the magnetic configuration to a double cusp eliminated the fast component. In the mirror configuration MNX helicon source, Cohen et al. ${ }^{10}$ showed that within the mirror regions the ions have a subthermal axial drift and outside the mirrors coils, a supersonic fast ion population appears at low pressure. Also in MNX, Sun et al. ${ }^{11}$ showed that, contrary to expectations for a Laval-nozzle magnetic configuration, the fast ion speed decreases as the nozzle magnetic field is increased. These observations reflect a strong correlation between the magnetic field profile, absolute magnetic field strength, and the downstream parallel ion velocity. 
Given the fact that all three possible acceleration mechanisms: electric double layer, geometric expansion, and magnetic moment conservation, may coexist and interact in the expanding region of the helicon plasma source, understanding their individual impact on ion acceleration is an important first step towards developing a complete understanding of ion acceleration in such systems. Because an external axial magnetic field of up to few hundred Gauss is required for propagation of a helicon wave in a gaseous plasma, by their very definition, helicon sources are magnetized plasma systems. Thus, at the junction between any helicon source and a downstream expansion/diffusion chamber, a divergent axial magnetic field naturally arises. Experimental investigations have demonstrated that the gradient in the axial magnetic field strength is intimately related to the potential drop across the electric double layer formed at the end of the source. ${ }^{13}$ Contrary to the helicon EDL model proposed by Chen, ${ }^{12}$ which predicts a spatial location of the EDL in the expansion region where the plasma radius expands by $28 \%$, experimental investigations in the HELIX-LEIA system found that the EDL spontaneously forms near the source-diffusion chamber junction, just inside the source and where the magnetic field gradient is largest. ${ }^{13}$ Similar distances between location of the EDL and the peak value of the magnetic field gradient have been reported for other helicon source-expansion chamber experiments: $\sim 5 \mathrm{~cm}$ for Chi-Kung, ${ }^{14} \sim 2 \mathrm{~cm}$ for MNX, ${ }^{15} 5-10 \mathrm{~cm}$ for WOMBAT, ${ }^{16}$ and most recently $7-9 \mathrm{~cm}$ in the Njord device. Only in the Njord device was the EDL found to appear in the expansion chamber. ${ }^{17}$

The magnetic field gradient is not the only aspect of the magnetic field structure that impacts the appearance of the EDL in an expanding helicon source. Charles [14] reported that EDL formation in the Chi-Kung helicon source is triggered by the magnetic field profile and strength in the source; below a threshold value of the magnetic field in the source the EDL doesn't form. For the same helicon source, Keesee et al. ${ }^{18}$ reported that potential drop across the EDL increases with increasing magnetic field strength in the source: the fast ion parallel flow speed increased from $2.5 \mathrm{~km} / \mathrm{s}$ to $5 \mathrm{~km} / \mathrm{s}$ as the magnetic field in the Chi-Kung source increased from $50 \mathrm{G}$ to $140 \mathrm{G}$. No change in the fast ion flow speed was observed when the magnetic field profile at the end of the source was varied. Recently, Charles and Boswell ${ }^{19}$ also demonstrated the existence of a threshold value of the magnetic field strength in the source of approximately $50 \mathrm{G}$ in Chi- 
Chapter 1: Introduction

Kung. In contrast to the Chi-Kung observations, Biloiu et al. [6] reported a significant increase in the speed of the fast ion group as the divergence in the expansion region (the ratio of the magnetic field strengths in the source and the diffusion chamber) increased in the HELIX-LEIA system. In those experiments, a maximum ion speed of $\sim 2.9 c_{s}$ was obtained for a magnetic field strength ratio of 43.

Although the experimental results described so far clearly demonstrate that the expanding magnetic field gradient and magnetic field strength in the source play an important role in defining the characteristics of the EDL, other observations suggest that pure geometrical expansion effects are also important. For example, the EDL only appears below a threshold pressure of 1-2 mTorr and the EDL strength scales with the gas pressure, i.e., the potential drop across the EDL increases as the pressure decreases [16].

Although recent experimental, computational, and theoretical studies have managed to clarify many key aspects of the helicon EDL formation process, some questions remain. ${ }^{20,21}$ For example, through which external control parameters can the EDL strength, thickness, and shape be varied? What is the role of the magnetic field profile in EDL formation and strength? What is the time scale for EDL formation? And based on Bohm criterion at the EDL edge, can the speed of one ionic component be increased at the expense of the speed of other components by using a mixture of gases?

The two measurable parameters of interest in studies of EDL physics are the ion velocity (energy) distribution function before and after the ions pass through the EDL and the value of the plasma potential throughout the EDL region. One method for measuring the ivdf in plasma is laser induced fluorescence (LIF). Unlike the retarding field energy analyzer (RFEA) method, which requires corrections for the perturbation of the ivdf created by the sheath in the front of the RFEA, ${ }^{22}$ LIF is non-intrusive. Since its first application to plasma diagnosis three decades ago by Stern and Johnson, ${ }^{23}$ both the technology employed for LIF measurements and the methods used to analyze LIF data have improved, ${ }^{24,25,26}$ e.g., tomographic inversions are now routinely used to obtain twodimensional velocity space measurements at a single spatial location ${ }^{27,28}$ and LIF is applied to many types of plasma discharges and for a wide range of experimental conditions. ${ }^{29}$ The magnitudes and directions of ambient electric and even weak magnetic 


\section{Chapter 1: Introduction}

fields in plasmas can also be determined from their effects on the energy levels of the specific quantum states (probed with LIF) of the target particle. ${ }^{30,31}$ Since the probing laser line width (typically $\approx 1 \mathrm{MHz}$ in the case of diode lasers and $\approx 100 \mathrm{MHz}$ in the case of dye lasers $)$ is much smaller than the particle absorption linewidth $(\approx 1 \mathrm{GHz}$ for particles of $M=40 \mathrm{amu}$ at room temperature and larger for higher temperatures), high resolution measurements of bulk velocity and temperature can be obtained. For a wide range of plasma conditions, Doppler broadening dominates over other line broadening mechanisms, e.g., Zeeman splitting, Stark broadening, and power broadening. Even in strongly magnetized discharges, for which Zeeman splitting cannot be ignored, choice of a particular polarization of the pumping laser often reduces the complexity of the measured absorption linewidth to a manageable level while also providing a direct measure of the ambient magnetic field strength. The spatial resolution of LIF measurements, determined by the intersection of the probing laser beam and the fluorescence collection optical path, is typically on the order of few $\mathrm{mm}^{3}$. The minimum time resolution of a LIF measurement is set by the lifetime of the upper quantum level of the pumped transition, usually on the order of a few nanoseconds. Therefore, all repetitive phenomena with a characteristic time larger than a few nanoseconds are amenable to be investigated by LIF. In practice, however, the time resolution is limited by the need to collect a sufficient number of LIF emission photons for reasonable signal to noise; the RC time constants of cables; the signal acquisition time requirements of the available electronics; and the particular plasma conditions. The main drawback of LIF is that the measured ivdf is that of the population of a certain excited state (for instance, for the 3 level LIF scheme $3 \mathrm{~d}^{2} \mathrm{G}_{9 / 2} \rightarrow 4 \mathrm{p}{ }^{2} \mathrm{~F}_{7 / 2} \rightarrow 4 \mathrm{~s}{ }^{2} \mathrm{D}_{5 / 2}$ usually employed for $\mathrm{Ar}^{+}$ investigations, it is the metastable state $3 \mathrm{~d}^{2} \mathrm{G}_{9 / 2}$ ) and not the entire ion population. However, it has been shown that for this $\mathrm{Ar}^{+}$state, in low pressure helicon plasma, the main population mechanism is excitation by electron impact from the ion ground state. Under these conditions, the population of the $3 \mathrm{~d}^{2} \mathrm{G}_{9 / 2}$ metastable level is proportional to $n_{e}{ }^{2} T_{e}{ }^{1 / 2}$ (where $n_{e}$ is the plasma density and $T_{e}$ is the electron temperature). ${ }^{32}$ Although not absolutely calibrated, this scaling law provides a qualitative correlation between the LIF signal and the total ion density. That the excited state ivdf basically is an accurate representation of the complete ivdf was demonstrated by Severn et al. ${ }^{33}$ They shown that 


\section{Chapter 1: Introduction}

same Ar ivdf can be obtained by employing different LIF schemes which probe three different $\mathrm{Ar}^{+}$excited levels (besides the LIF scheme discussed in this work for which a dye laser is necessary, the other two LIF schemes $3 \mathrm{~d}^{4} \mathrm{~F}_{9 / 2} \rightarrow 4 \mathrm{p}^{4} \mathrm{D}^{0}{ }_{7 / 2} \rightarrow 4 \mathrm{~s}{ }^{4} \mathrm{P}_{5 / 2}$ and $3 \mathrm{~d}$ ${ }^{4} \mathrm{~F}_{7 / 2} \rightarrow 4 \mathrm{p}^{4} \mathrm{D}^{0}{ }_{5 / 2} \rightarrow 4 \mathrm{~s}{ }^{4} \mathrm{P}_{3 / 2}$ were probed with a diode laser).

Since the controlled acceleration of ions without the use of grids or externally imposed potentials has immediate applicability to the development of plasma thrusters and plasma processing sources, a complete understanding of the mechanism responsible for ion acceleration in a divergent magnetic field, expanding plasma source, is of importance for basic and applied plasma physics. The results presented in this dissertation are based on the investigation of ion behavior (acceleration and heating) in single (argon) and multi-component (argon + xenon and argon + helium) helicon plasmas. The experiments were carried out in HELIX (Hot hELIcon eXperiment) - LEIA (Large Experiment on Instabilities and Anisotropies) system. In this helicon plasma source - diffusion chamber system, for neutral pressures below $1.5 \mathrm{mTorr}$ (when the ion mean free path length is comparable with the scale length of the magnetic field gradient), a localized, current-free double layer develops. Ion acceleration occurs at the end of the helicon source and appears to result from EDL formation and classic magnetic expansion. Laser Induced Fluorescence (LIF) provided ion velocity distribution function (ivdf) measurements at different spatial location, for different magnetic expansion configurations, under different operating conditions, and for pure argon and mixed gases (argon with lighter (He) or heavier (Xe) gas). From one dimensional (1D) LIF measurements, the parallel $\mathrm{Ar}^{+}$and $\mathrm{Xe}^{+}$flow speeds and temperatures upstream and downstream of the EDL were determined. 2D LIF was employed to obtain measurements of the parallel and perpendicular ivdf downstream the EDL. Time resolved LIF measurements were used to investigate the development phase of the EDL as well as its temporal stability. In conjunction with Langmuir probe (LP) and optical emission spectroscopy (OES) measurements and some theoretical models, these LIF measurements have been able to provide a clearer picture of the EDL formation in helicon plasma.

Besides these introductory remarks, this dissertation contains seven additional chapters. A discussion of the classic plasma sheath, the classic electric double layer, the generalized Bohm criterion for sheath and EDL existence, and a general model of EDLs 
Chapter 1: Introduction

in multi-component plasmas is given in Chapter 2. A brief introduction into the physics of helicon plasma sources, a description of HELIX-LEIA system, and the typical plasma parameters is given in Chapter 3. Chapter 4 describes the diagnostic methods used, including: the Langmuir probe and the Druyvesteyn differentiation method of obtaining the electron energy distribution function; a model used to estimate the species ion density in a two component plasma from spectral emission lines of ions and neutrals; the 1D and 2D laser induced fluorescence techniques for stationary and pulsed plasmas; and a description of a scanning probe used for LIF tomography. Experimentally obtained characteristics of two-ion component plasma and the effect of the second species on supersonic ion acceleration are described in detail in Chapter 5. In Chapter 6, experimental measurements of the temporal evolution of the bimodal ion velocity distribution function (ivdf) in pulsed argon plasma are presented. The two-dimensional (2D) argon ivdfs observed in the expansion region of the helicon plasma source, as well as a discussion of the interpretation of LIF data obtained at oblique laser injection, are presented in Chapter 7. A summary and conclusion of the insights into ion acceleration in expanding helicon plasmas gleaned from the measurements is given in Chapter 8 . 


\section{Chapter 1 References}

${ }^{1}$ C. Charles and R. W. Boswell, Appl. Phys. Lett. 82, 1356 (2003)

${ }^{2}$ S. A. Cohen, N. S. Siefert, S. Stange, R. F. Boivin, E. E. Scime, and F. M. Levinton, Phys. Plasmas 10, $2593(2003)$

${ }^{3}$ X. Sun, C. Biloiu, R. Hardin, and E. Scime, Plasma Sources Sci. Technol. 13, 359 (2004)

${ }^{4}$ C. Charles, R. W. Boswell and M. A. Lieberman, Appl. Phys. Lett. 89, 261503 (2006)

${ }^{5}$ N. Sadeghi, T. Nakano, D. J. Trevor, and R. A. Gottscho, J. Appl. Phys. 70, 2552 (1991)

${ }^{6}$ I. A. Biloiu, E. Scime, and C. Biloiu, Appl. Phys. Lett. 92, 191502 (2008)

${ }^{7}$ W. M. Manheimer and R. F. Fernsler, IEEE Trans. Plasma Sci. 29, 75 (2001)

${ }^{8}$ C. S. Corr, J. Zanger, R. W. Boswell, and C. Charles, Appl. Phys. Lett. 91, 241501 (2007)

${ }^{9}$ T. Nakano, K. P. Giapis, R. A. Gottscho, T. C. Lee, and N. Sadeghi, J. Vac. Sci. Technol. B 11, 2046 (1993)

${ }^{10}$ S. A. Cohen, X. Sun, N. M. Ferraro, E. Scime, M. Miah, S. Stange, N. Siefert, R. F. Boivin, IEEE Trans. Plasma Sci. 34, 792 (2006)

${ }^{11}$ X. Sun, S. A. Cohen, E. E. Scime, and M. Miah, Phys. Plasmas 12, 103509 (2005)

${ }^{12}$ F. F. Chen, Phys. Plasmas 13, 034502 (2006)

${ }^{13}$ X Sun, A. M. Keesee, C. Biloiu, E. E. Scime, A. Meige, C. Charles, and R. W. Boswell, Phys. Rev.Lett. 95, 025004 (2005)

${ }^{14}$ C. Charles, Phys. Plasmas 12, 044508 (2005)

${ }^{15}$ X. Sun, S.A. Cohen, E. E. Scime, and M. Miah, Phys. Plasmas 12, 103509 (2005)

${ }^{16}$ O. Sutherland, C. Charles, N. Plihon, and R. W. Boswell, Phys. Rev. Lett. 95, 2050002 (2005)

${ }^{17}$ H. S. Byhring, C. Charles, A. Fredriksen, and R. W. Boswell, Phys. Plasmas 15, 102113 (2008)

${ }^{18}$ A. M. Keesee, E. E. Scime, C. Charles, and R. Boswell, Phys. Plasmas 12, 093502 (2005)

${ }^{19}$ C. Charles and R. W. Boswell, Appl. Phys. Lett. 91, 201505 (2007)

${ }^{20}$ M. A. Lieberman and C. Charles, Phys. Rev. Lett. 97, 045003 (2006)

${ }^{21}$ C. Charles, Plasma Sources Sci. Technol. 16, R1 (2007)

${ }^{22}$ Z. Harvey, S. Chakraborty Thakur, A. Hansen, R. Hardin, W. S. Przybysz, and E. E. Scime, Rev. Sci. Instrum. 79, 10F314 (2008)

${ }^{23}$ R. A. Stern and J. A. Johnson III, Phys. Rev. Lett. 34, 1548 (1975)

${ }^{24}$ D. H. Hill, S. Fornaca, and M. G. Wickham, Rev. Sci. Instrum. 54, 309 (1983)

${ }^{25}$ M. J. Goecker and J. Goree, J. Vac. Sci. Technol. A 7, 977 (1989)

${ }^{26}$ A. M. Keesee, E. E. Scime, and R. Boivin, Rev. Sci. Instrum. 75, 4091 (2004)

${ }^{27}$ C. Biloiu, E. Scime, and B. McGeehan, Rev. Sci. Instrum. 75, 4296 (2004)

${ }^{28}$ D. C. Zimmerman, R. McWilliams, and D. A. Edrich, Plasma Sources Sci. Technol. 14, 581 (2005)

${ }^{29}$ J. Amorim, G. Baravian, and J. Jolly, J. Phys. D: Appl. Phys. 33, R51 (2000) 
${ }^{30}$ E. Foley and F. Levinton, Rev. Sci. Instrum. 75, 3462 (2004)

${ }^{31}$ W. Noonan, T. Jones, and P. Ottinger, Rev. Sci. Instrum. 68, 1032 (1997)

${ }^{32}$ E. E. Scime, R. Hardin, C. Biloiu, A. M. Keesee, and X. Sun, Phys. Plasmas 14, 043505 (2007)

${ }^{33}$ G. D. Severn, D. A. Edrich, and R. McWilliams, Rev. Sci. Instrum. 69, 10 (1998) 


\section{Chapter 2: Plasma Sheaths and Double Layers}

\subsection{Plasma - Wall Interaction}

The plasma - wall interface plays a central role in much of plasma physics and remains one of the oldest open questions of fundamental plasma science. ${ }^{1}$ The term sheath was introduced to characterize the spatial region that separates the bulk neutral plasma from the bounding wall. According to Langmuir's original definitions, "There will be therefore a layer of gas near the electrode where there are positive ions but no electrons, and in this region there will therefore be a positive ion space charge. The outer edge of this sheath of ions will have a potential of -1 and the positive ions pass through this outer edge with a velocity corresponding to 2 volts." 2 and "Except near the electrodes where there are sheaths containing very few electrons, the ionized gas contains ions and electrons in about equal numbers so that the resultant space charge is very small. We shall use the name plasma to describe this region containing balanced charges of ions and electrons" 3 For an electropositive plasma, the interaction of plasma with a conducting wall, in its simplest form, can be characterized as follows: due to the high mobility of the electrons, the plasma develops an excess of positive charge and the plasma potential rises to a positive value with respect to the surrounding walls. The repulsion of electrons by the plasma-wall potential difference results in the formation of a positive space charge region (sheath), shielding the neutral plasma from the negative wall. The sheath edge location and the sheath width are a function of the electron temperature, the electron Debye length $\lambda_{D}$, and the characteristic length $L$ of the plasma (the distance from the plasma center to the wall). The interchange of physical mechanisms dominating the bulk plasma and the sheath regions results in a mathematical singularity in the plasma equations at the sheath edge. In the asymptotic limit $\lambda_{D} / \mathrm{L} \rightarrow 0$ this singularity can be removed by requiring that the plasma equations fulfill the "Bohm criterion", ${ }^{4}$ i.e., the ions enter the sheath region with a large velocity that exceeds the thermal ion motion. Consequently, the classic sheath model also includes ion acceleration by an electric field extending over a "presheath" region. The origins of the mathematical singularity; the extent of the presheath region; the effect of the type of plasma, dc or rf, 
on the sheath; and the nature of the sheath, collision dominated or collisionless, are subjects that have garnered considerable interest and generated scientific disputes throughout the history of plasma physics. ${ }^{5,6,7}$

\subsection{The Bohm Criterion for Single Ion Species Plasma}

In a low pressure electropositive gas discharge characterized by an electron temperature much higher than ion temperature, it is well established that quasineutrality is violated near the boundary walls that are in contact with the plasma. Considering a neutral, unmagnetized plasma with one type of singly-charged ions, the electron flux ( $\Gamma_{e}$ $\left.=n_{e}\left\langle v_{e}\right\rangle / 4\right)$ to a bounding wall is much higher than the ion flux $\left(\Gamma_{i}=n_{i}\left\langle v_{i}\right\rangle / 4\right)$ due to the difference between electron and ion mobilities.

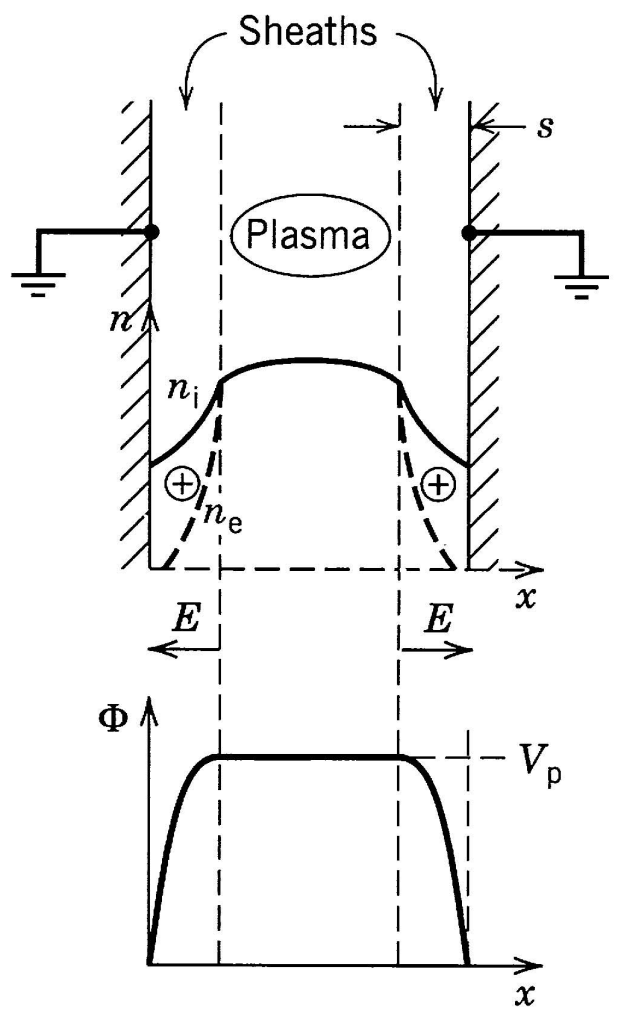

Figure 2.1. Schematic of the planar sheaths that build-up between plasma and grounded, conducting walls. ${ }^{8}$

Here $n_{e, i}$ and $\left\langle v_{e, i}\right\rangle$ are the number density and mean speed for electrons and ions, respectively. Balance of the electron and ion losses and consequently bulk plasma quasineutrality is realized by self-adjustment of the plasma potential $V_{p} ; V_{p}$ becomes 
positive with respect to the boundary surface (see Figure 2.1). The result is the establishment of an electric field, $E$, pointing towards the boundary wall and extending over a distance of few electron Debye lengths. Since the electric field reflects low energy electrons back towards the plasma, in the region adjacent to the bounding wall, the ion density exceeds the electron density and a positive space-charge develops. For the given discharge parameters, this space-charge region is a thin, collisionless, planar sheath.

To obtain quantitative descriptions of the potential drop across the sheath and the sheath width, we start from Poisson's equation:

$$
\frac{d^{2} \Phi}{d x^{2}}=-\frac{e}{\varepsilon_{0}}\left(n_{i}-n_{e}\right),
$$

where $\Phi$ is the space potential, $e$ the elementary charge, and $\varepsilon_{0}$ dielectric permittivity of vacuum. Assuming a Maxwellian distribution of electrons with an electron temperature $T_{e}$ and in Boltzmann equilibrium, the electron density in the sheath is

$$
n_{e}=n_{s} \exp \left(\frac{e \Phi}{k_{B} T_{e}}\right)
$$

where $n_{s}$ and $k_{B}$ are the electron density in the bulk plasma (sheath edge) and Boltzmann's constant respectively. Introducing the ion continuity and ion momentum equations

$$
\frac{d u_{i}}{d x}+\frac{u_{i}}{n_{i}} \frac{d n_{i}}{d x}=0
$$

and

$$
M u_{i} \frac{d u_{i}}{d x}=-e \frac{d \Phi}{d x}-\gamma k_{B} T_{i} \frac{d n_{i}}{d x},
$$

where $u_{i}, n_{i}$, and $T_{i}$ are the ion speed, density and temperature, $M$ is the ion mass and $\gamma$ is the adiabatic exponent, we arrive at the expression for the ion velocity at the sheath edge, i.e., the Bohm criterion

$$
M u_{s}^{2} \geq k_{B}\left(T_{e}+\gamma T_{i}\right) .
$$

For a low temperature plasma $\left(T_{e}>>T_{i}\right)$, the Bohm criterion reduces to ${ }^{9}$

$$
u_{s} \geq u_{B}=\left(\frac{k_{B} T_{e}}{M}\right)^{1 / 2} .
$$




\section{Chapter 2: Plasma Sheaths and Double Layers}

Balancing the electron flux $\Gamma_{e}=n_{s}\left\langle v_{e}\right\rangle \exp \left(e \Phi / k_{B} T_{e}\right) / 4$ and ion flux $\Gamma_{i}=$ $n_{s}\left(k_{B} T_{e} / M\right)^{1 / 2}$ at the wall, the potential drop across the sheath is

$$
\Phi=\frac{k_{B} T_{e}}{2 e} \ln \left(\frac{M}{2 \pi m}\right) .
$$

The current density for space-charge limited ion current flowing between two planes with a potential difference $\Phi$ separated by a distance $s$ is given by the Child-Langmuir law

$$
J=\frac{4 \varepsilon_{0}}{9}\left(\frac{2 e}{M}\right)^{1 / 2} \frac{\Phi^{3 / 2}}{s^{2}}
$$

The current density at the sheath edge is

$$
J=e n_{s} u_{B} .
$$

Equating (2.7) and (2.8), the sheath thickness assuming space charge limited current is

$$
s=\frac{2^{1 / 2}}{3} \lambda_{D}\left(\frac{2 e \Phi}{k_{B} T_{e}}\right)^{3 / 4},
$$

where $\lambda_{D}=\sqrt{\varepsilon_{0} k_{B} T_{e} / e^{2} n_{s}}$ is the electron Debye length.

These equations for the sheath potential drop and sheath thickness are generally valid for the simple case of dc discharges. In the case of $\mathrm{rf}$ discharges, although the rf fields oscillate at the driving frequency, it has been established that the Bohm criterion is still relevant at the plasma edge - even though the position of the plasma edge varies in time. For the usual case of the driving frequency exceeding the ion plasma frequency but being smaller than electron plasma frequency $\omega_{p e}>\omega>\omega_{p i}$, the motion of ions and electrons can be considered separately. Typically, the ions are described by their time averaged behavior and the full electron response to the oscillating rf field is considered. The resulting sheath solution periodically expands and contracts at the rf driving frequency. ${ }^{10}$ It has been shown that the ratio of sheath oscillation amplitude to the Debye length scales with the ratio of the oscillating rf current to the dc ion current through the sheath times the ratio of the ion plasma frequency to the rf driving frequency. ${ }^{11,12,13}$

For plasma immersed in an external magnetic field, the sheath structure depends on the magnetic field orientation relative to the wall. For a magnetic field perpendicular to the bounding wall, charged particle motion is not affected and the sheath structure is similar to the unmagnetized case. For a magnetic field parallel or oblique to the bounding 
wall, depending on the relative magnitude of the electron Debye length compared to the electron gyroradius, a magnetic presheath may form. If the magnetic field is weak and the plasma density is large, so that the electron Debye length is smaller than the electron gyroradius, the electrons are still easily lost to the wall and an electrostatic Debye sheath forms. In contrast to the simple unmagnetized case, the presheath width is on the order of ion gyroradius and the electron energy distribution function is far from Maxwellian. Increasing the magnetic field strength, the electron gyroradius becomes smaller and the electron is trapped in the presheath by a sufficiently strong magnetic field. Therefore, electron movement to the wall is inhibited, the wall potential is reduced, and a less

pronounced positive space charge region is created in front of the wall. Such a magnetized presheath or Chodura layer is discussed in detail in Ref. [14]. Particle motion in the magnetic presheath must be described by the full kinetic equations. Therefore, the expressions for the particle fluxes to the bounding wall as function of potential difference are more complicated for magnetized systems with magnetic fields parallel to the bounding walls.

For the physical phenomena of interest occurring in the HELIX-LEIA system in this work, the magnetic field is either normal to the imaginary separation surface between the helicon source and diffusion chamber or, in the case of the expanding plasma it is a very weak field. Thus, in this work, the simple sheath model, i.e., the unmagnetized sheath is assumed throughout.

\subsection{The Bohm Criterion for Two-Positive Ion Species Plasma}

Many plasmas of practical interest contain more than one species of positive ions. Since the ions differ in mass and therefore in their mobilities, there is a need to understand the structure of the plasma-wall interaction for multiple ion plasmas. Following a recent analytical and computational model for a plasma that contains two positive ion species, ${ }^{15}$ we assume that ionization occurs only by direct electron impact and that the friction term in the ion momentum transfer equation can be described by a constant effective collision frequency. For simplicity, only the one-dimensional case is considered. Beginning with the plasma quasineutrality condition 


$$
n_{e} \cong n_{1}+n_{2} \text {, }
$$

which defines the total electron density as a sum of the two singly charged ion densities $n_{1}$ and $n_{2}$, the particle balance equations for each ion species become:

$$
\frac{d}{d x}\left(n_{1} v_{1}\right)=n_{e} Z_{1}=\left(n_{1}+n_{2}\right) Z_{1}
$$

and

$$
\frac{d}{d x}\left(n_{2} v_{2}\right)=n_{e} Z_{2}=\left(n_{1}+n_{2}\right) Z_{2},
$$

where $v_{1,2}$ and $Z_{1,2}$ are the ion speed and ionization rate of gas species 1 and 2, respectively. From the ion flux equations $n_{1} v_{1} / n_{2} v_{2}=Z_{1} / Z_{2}$, momentum conservation for each ion species is:

$$
\frac{d}{d x}\left(n_{1} v_{1}^{2}\right)+n_{1} v_{1} v_{12}+\frac{e n_{1}}{M_{1}} \frac{d \Phi}{d x}=0
$$

and

$$
\frac{d}{d x}\left(n_{2} v_{2}^{2}\right)+n_{2} v_{2} v_{21}+\frac{e n_{2}}{M_{2}} \frac{d \Phi}{d x}=0,
$$

where $M_{1,2}$ are the ion masses, $\Phi$ is the sheath potential, $v_{12}$ is the effective collision frequency of ion 1 moving in a gas mixture containing a fraction $f_{2}$ of species 2 and $v_{21}$ is the corresponding collision frequency for ion 2 . In the sheath, the electrons obey a Boltzmann distribution

$$
n_{e}=n_{e s} e^{e \Phi / k_{B} T_{e}}=\left(n_{1 s}+n_{2 s}\right) e^{e \Phi / k_{B} T_{e}},
$$

where the subscript $s$ denotes the sheath edge.

Differentiating Eq. (2.13) with respect to $x$ yields $d \Phi / d x$ in terms of $d n_{1} / d x$ and $d n_{2} / d x$. After considerable algebraic manipulation, we obtain four first order differential equations for $n_{1}, n_{2}, v_{1}$, and $v_{2}$. The determinant of these equations vanishes where

$$
n_{1}+n_{2}=n_{1} \frac{c_{1}^{2}}{v_{1}^{2}}+n_{2} \frac{c_{2}^{2}}{v_{2}^{2}},
$$

with $c_{j}^{2}=k_{B} T_{e} / M_{j}, j=1,2$ being the $j$-th ion sound speed (the Bohm speed). Eq. (2.14) is the generalized Bohm criterion for a collisionless sheath in a low temperature plasma with two positive ion species. ${ }^{16}$ Note that when either $n_{1}$ or $n_{2}$ is zero in Eq. (2.14), the expression reduces to the one species Bohm criterion. Furthermore, it follows from Eq. 
(2.14) that either both ions obey their individual Bohm criteria (enter the sheath at their individual sound speeds) or that one species has a speed above its ion sound speed and the other has a speed below it. From the mathematics, there is no way to determine $a$ priori which solution is valid.

\subsection{Sheath and Electric Double Layer Analogies and Differences}

An electric double layer (EDL) is a term generally applied to a region in a plasma where two thin layers of opposite electric charge coexist in close proximity. The two sides of the double layer are generally referred as the upstream (high potential side) and downstream (low potential side) regions. The electrostatic potential difference between the two layers, separated by tens to hundreds Debye lengths, gives rise to a spatially localized electric field which accelerates ions (electrons) from the high potential (low) to the low potential (high) side. The acceleration of ions and electrons in opposite directions is one of the fundamental differences between an EDL and a sheath. In nature, it is the acceleration of charged particles that is often the most visible manifestation of an EDL. For example, strong, quasistable, magnetic-field aligned, EDLs having electric fields of $0.1 \mathrm{~V} / \mathrm{m}$ and spanning $\sim 10$ Debye lengths are found in the auroral zone. ${ }^{17}$ The EDLs are oriented with their high potential closest towards the Earth, so electrons are accelerated towards the Earth while ions are accelerated upwards toward ionosphere. ${ }^{18,19,20}$ In the lab, EDLs are created through a variety of methods, including: injection of ion or electron beams into a plasma; ${ }^{21,22}$ bringing in contact two plasmas of different electron temperatures and implicitly different potentials; ${ }^{23}$ plasma expansion into vacuum or in a

diverging magnetic field. ${ }^{24,25,26}$ Depending on the specific plasma conditions, EDLs may differ in their shape, 2D or 3D structure, size, strength, magnetization, stability, and current-driven or current-free nature. The second characteristic that distinguishes a bounding wall sheath from an EDL is that an EDL can form anywhere in the plasma; unlike an electrostatic sheath which is fixed to the surface of an object immersed in the plasma or the plasma boundary. 


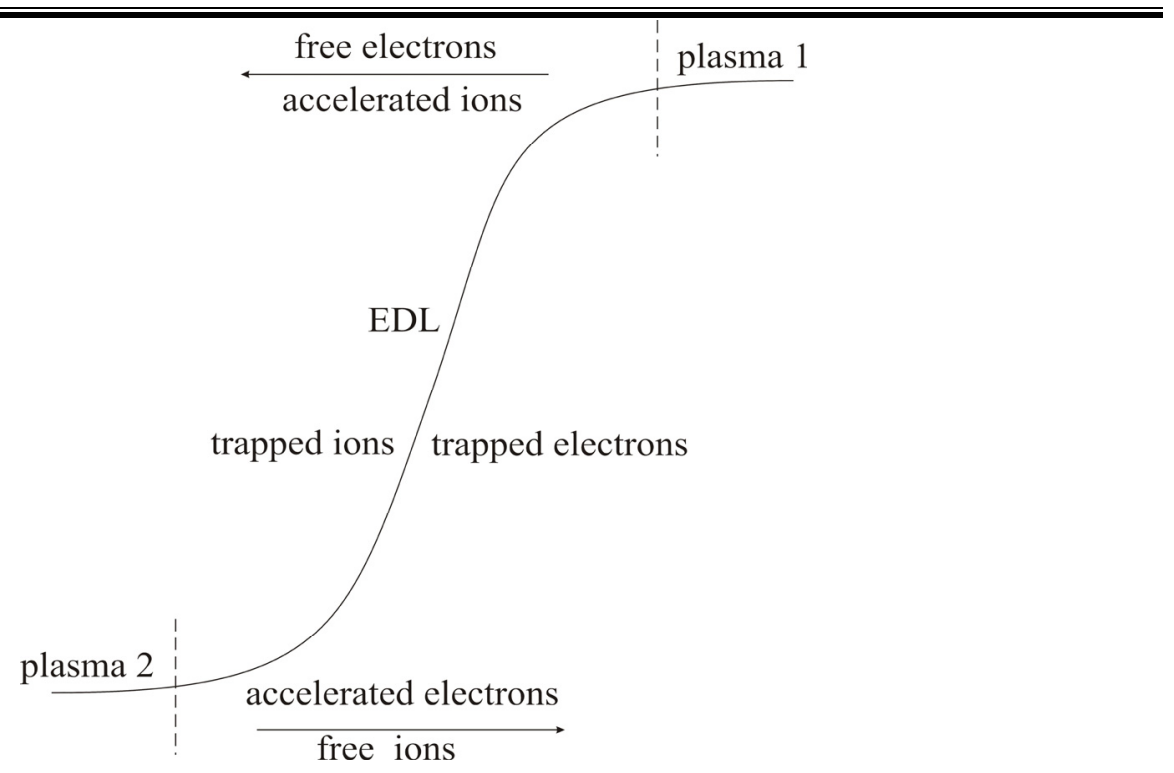

Figure 2.2. Schematic of an electric double layer (EDL) formed between two plasmas (vertical dashed lines indicate the plasma boundaries) with different plasma potentials

For the general case of an EDL formed between two adjacent plasmas at different potentials (see Figure 2.2), there is a population of trapped (or reflected) low energy electrons on the high potential side (right) of the EDL, a population of trapped (or reflected) low energy ions on the low potential side (left) of the EDL, a population of electrons having enough energy to overcome the EDL potential barrier and pass from the high potential side to low potential side (called free electrons), and a population of high energy ions which overcome the EDL potential barrier (called free ions) and pass from left to right, i.e., from the low potential side of the EDL to the high potential side of the EDL. ${ }^{27}$ The two additional populations, an energetic electron beam and an energetic ion beam on the high potential side and the low potential side, respectively, result from acceleration as the charged particles transit the EDL. ${ }^{28}$ As shown in Figure 2.3, all four particle populations are easily distinguished in phase space. At $x \rightarrow-\infty$ for ions and $x \rightarrow$ $+\infty$ for electrons, the accelerated populations (hatched areas) coming from upstream and downstream, respectively, are completely distinct from the background populations. At the edges of the EDL there could be, in principle, a discontinuity in the phase space densities. In reality, however, any discontinuities are eliminated by fluctuations and diffusion. ${ }^{29}$ 


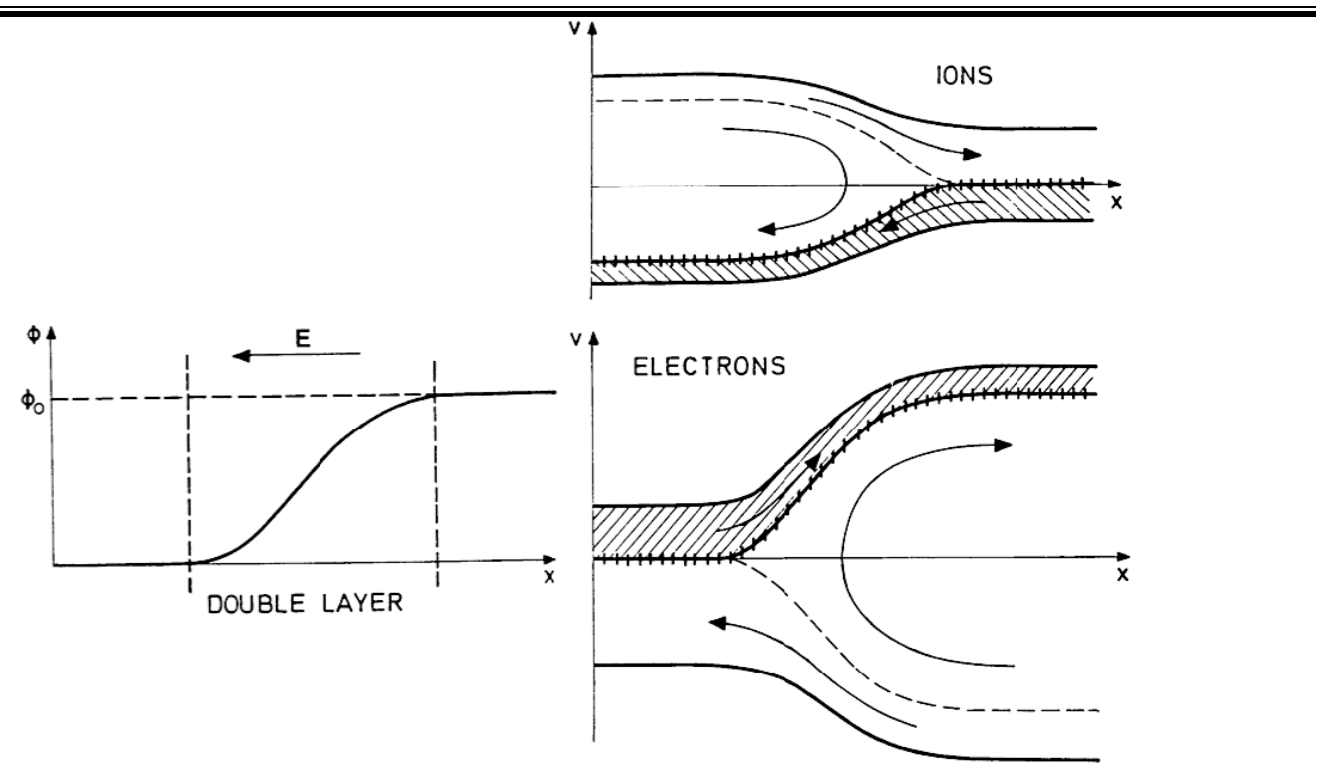

Figure 2.3. Potential distribution $\phi(x)$, ion and electron phase space distributions across an EDL with potential drop $\phi_{0}$ and internal electric field $\mathrm{E}$. The hatched areas indicate accelerated populations. The reflection of ions and electrons within the separatrices (symmetrically placed broken and barred curves) are indicated by the arrows showing velocity reversal.

The most important characteristic of an EDL is its strength, given by the ratio of the potential difference across the EDL to the plasma thermal potential $e \phi_{D L} / k_{B} T_{e}$, with $T_{e}$ being the electron temperature on the low potential side. ${ }^{30}$ An EDL is considered as weak if this ratio is less than 10 and strong if this ratio exceeds 10 . The weaker the EDL, the larger the fraction of background particles that will be able to surmount the potential barrier.

Another important characteristic of an EDL is magnitude of the net current flowing through the EDL. The net current flowing through the EDL is given by the balance of passing populations (free electrons and ions) and accelerated populations (ions flowing downstream and electrons flowing upstream). Generally, to maintain the current through an EDL, an external power source is required to close the current loop; as is the case of EDLs produced in double plasma sources, triple plasma sources, and Q machines. ${ }^{31}$ However, under certain conditions it is possible that the net current through the EDL is zero. Such EDLs are called current-free double layers. 


\subsection{Theoretical Description of a Plasma EDL: the Sagdeev Potential}

To exist in a plasma, an EDL must fulfill three simultaneous conditions: ${ }^{32}$ the potential drop must exceed the electron thermal energy; the electric field must be stronger in the EDL than outside; and the net charge must be zero. An analytical solution for the EDL potential structure requires solving the Poisson and Vlasov equations with the boundary conditions of a null electric field and zero net charge at the edges of the EDL. Since the mathematical representation is nonlinear, the analytic solution is difficult to obtain. A solution method that has been shown to be particularly suited for electric double layers (EDL) in various plasma conditions is the Sagdeev potential. ${ }^{33}$ For the simple one-dimensional (along the $z$-axis) two-ion species plasma case, Poisson's equation for the electrostatic potential $\phi$ is

$$
-\varepsilon_{0} \frac{d^{2} \phi}{d z^{2}}=\rho(z),
$$

where $\rho$ is the total charge density of the plasma. When it is possible to obtain the charge density as a functional of a potential $\rho(\phi)$, one can introduce the Sagdeev potential $V(\phi)$ defined as

$$
V(\phi)=\int_{\phi} \rho(\psi) d \psi
$$

Appying $\rho=d V / d \phi$, Poisson's equation can be integrated once to obtain

$$
\frac{\varepsilon_{0}}{2}\left(\frac{d \phi}{d z}\right)^{2}+V(\phi)=\Pi
$$

where $\Pi$ is a constant. $\Pi$ can be interpreted to represent the energy of a fictitious particle located at a "position" $\phi$, with a "velocity" $d \phi / d z$, and at the "time" $z$. Note that the Sagdeev potential has the dimensions of energy density. Imposing the constraint that the net charge $\rho(z)$ and the electric field $E=-d \phi / d z$ vanish at each side of the double layer, defining the potential across the double layer to be $\phi_{D L}$, and employing Eqs. (2.16) and (2.17), the Sagdeev potential must satisfy the conditions

$$
V^{\prime}(0)=V^{\prime}\left(\phi_{D L}\right)=0 \text { and } V(0)=V\left(\phi_{D L}\right)=\Pi,
$$


where $V^{\prime}$ is the first derivative of $V$ with respect to $\phi$. Eq. (2.17) also requires that the energy density is positive, i.e., $\varepsilon_{0} \mathrm{E}^{2} / 2=\Pi-V(\phi) \geq 0$. Thus, the Sagdeev potential must satisfy the inequality

$$
V(\phi) \leq \Pi
$$

At the double layer edges, $V(\phi)$ can be expanded in a Taylor series and to second order in $\phi$, the inequality of Eq. (2.19) becomes

$$
V^{\prime \prime}(0) \leq 0 \text {, and } V^{\prime \prime}\left(\phi_{D L}\right) \leq 0
$$

The necessary conditions for the formation of an electric double layer in a plasma are thus given by Eqs. (2.18) and (2.20).

The Sagdeev potential is obtained from the equations of motion of the plasma components. For electrons in Boltzmann equilibrium with the electrostatic field, the electron charge density, i. e. the derivative of the electron contribution to the Sagdeev potential $V_{e}(\phi)$, is

$$
\rho_{e}(z)=V_{e}^{\prime}(\phi)=-e n_{e 0} e^{e \phi / k_{B} T_{e}},
$$

where $n_{e 0}$ is the electron density at $\phi=0$. Integrating once, the electron contribution to the Sagdeev potential is

$$
V_{e}(\phi)=\Pi_{e}-n_{e 0} k_{B} T_{e} e^{e \phi / k_{B} T_{e}} .
$$

Examination of Eq. (2.22) suggests that the electron contribution to the Sagdeev potential is directly related to the electron pressure.

The pressure arising from the ions is assumed to be ignorable, i.e., small but finite ion temperature. This approximation is appropriate for low temperature plasmas where the ion temperatures are much smaller than the electron temperature. To express the total ion density as a function of the electrostatic potential, ion velocities are eliminated through the continuity $\left(n_{j} v_{j}=n_{j 0} v_{j 0}\right)$ and energy conservation $\left(M_{j} v_{j}{ }^{2} / 2+e \phi(z)=M_{j 0} v_{j 0}{ }^{2} / 2\right)$ equations for each species. Here $n, v, M$ denote the number density, fluid velocity, and ion mass. The subscript $j(j=1,2)$ again refers to the different plasma species and the index 0 refers to the locations where the potential must vanish. The positive ion charge density is then given by

$$
\rho_{j}(z)=V_{j}^{\prime}(\phi)=\sum_{j} e n_{j 0}\left(1-\frac{2 e \phi}{M_{j} v_{j 0}^{2}}\right)^{-1 / 2} .
$$


Thus, the ion contribution to the Sagdeev potential is

$$
V_{j}(\phi)=\Pi_{i}-\sum_{j} n_{j 0} M_{j} v_{j 0}^{2}\left(1-\frac{2 e \phi}{M_{j} v_{j 0}^{2}}\right)^{1 / 2},
$$

where $\Pi_{i}$, and $\Pi_{e}$ from Eq. (2.22), are integration constants. The total Sagdeev potential for an electropositive plasma with two ion species is then

$$
V\left(\Phi, \mathrm{M}_{j}\right)=\Pi+n_{e 0} k_{B} T_{e}\left(1-e^{\Phi}\right)+\sum_{j} n_{j 0} k_{B} T_{e} \mathrm{M}_{j}^{2}\left[1-\left(1-\frac{2 \Phi}{\mathrm{M}_{j}^{2}}\right)^{1 / 2}\right],
$$

where the dimensionless potential $\Phi=e \phi / k_{B} T_{e}$, the boundary condition $V(0)=\Pi$, and the definition of the ion Mach number $\mathrm{M}_{j}=v_{j} /\left(k_{B} T_{e} / M_{j}\right)^{1 / 2}$ have been used.

An example of the Sagdeev potential solution for a plasma double layer in the simple case of one ion species is shown in Figure 2.4 as a function of Mach number and the dimensionless potential defined above. From Figure 2.4 it is clear that the potential must be finely tuned such that the fictitious particle starting at rest at one maximum, rolls down the potential and comes to rest at the other maximum.

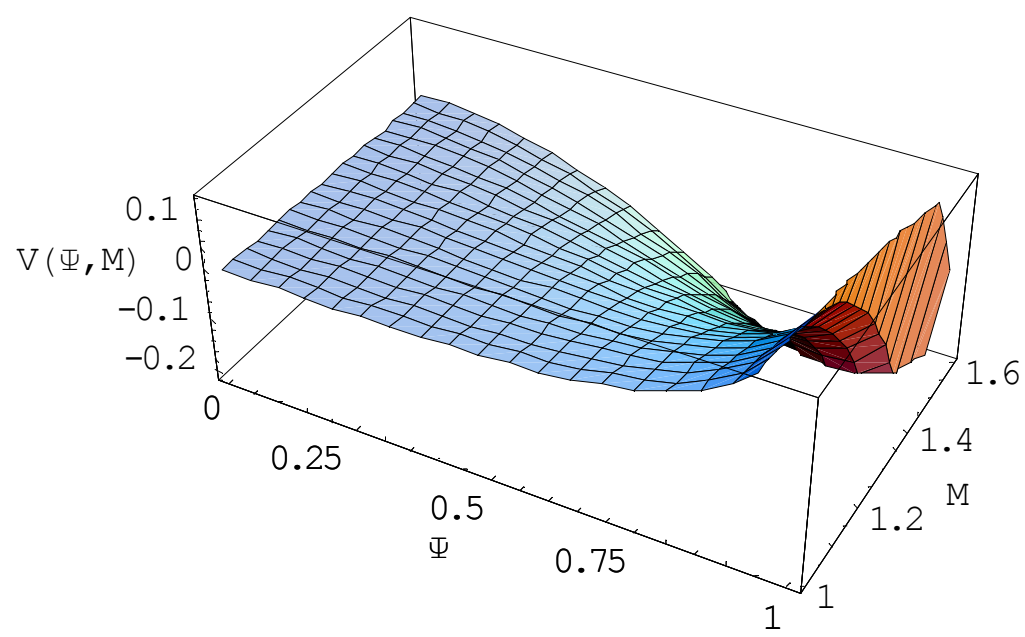

Figure 2.4. The Sagdeev potential for a single ion species plasma double layer.

With the charge densities defined, the two necessary conditions $V^{\prime}(0)=0$ and $V^{\prime \prime}(0) \leq 0$ have a simple physical interpretation. Combining Eqs. (2.21) and (2.23), equivalent expressions for the necessary conditions are obtained: 


$$
\begin{aligned}
& V^{\prime}(0)=0 \quad \Leftrightarrow \quad n_{e 0}=\sum_{j} n_{j 0} \\
& V^{\prime \prime}(0) \leq 0 \quad \Leftrightarrow \quad \sum_{j} \frac{n_{j 0}}{n_{e 0}} \frac{1}{\mathrm{M}_{j}^{2}} \leq 1
\end{aligned}
$$

The first condition is a requirement for plasma neutrality at one edge of the EDL. The second condition is identical to the generalized Bohm criterion for formation of wall sheaths (Eq. (2.14)). The two conditions $V\left(\phi_{D L}\right)=\Pi$ and $V^{\prime}\left(\phi_{D L}\right)=0$ lead to a second order equation with a root at $\phi_{D L}=0$, but no positive roots. Since, $V(\phi)$ cannot have a root on the domain $\phi>0$, all the necessary conditions for the formation of a double layer are not satisfied. Although the non-satisfaction of the necessary conditions excludes any double layer behavior, soliton solutions are still allowed for particular initial conditions. Thus, a plasma with Boltzmann electrons and a single species of cold positive ions cannot sustain a double layer. For the general case of multiple cold positive ion species, ${ }^{34}$ it has also been shown that double layers of arbitrary amplitude cannot exist in a plasma with one Boltzmann electron population, no matter how many cold ion species are present. Therefore, the formation of electric double layers in a plasma requires at least one finite temperature ion species.

Giving the difficulty of finding finite temperature ion and electron particle distribution functions that yield an EDL solution, it is often not possible to solve for the Sagdeev potential. For weak EDLs, the Sagdeev potential can be expanded in a power series and terms up to $\phi^{4}$ retained. In this limit, the EDL solution of Eq. (2.17) becomes

$$
\phi(z)=\phi_{D L}\left[1-\tanh \left(\frac{z}{d}\right)\right],
$$

where $d$ is the EDL thickness. As shown in Figure 2.5, such a potential is symmetric with respect of the middle of the EDL and the adjacent positive and negative space charges have equal widths. 


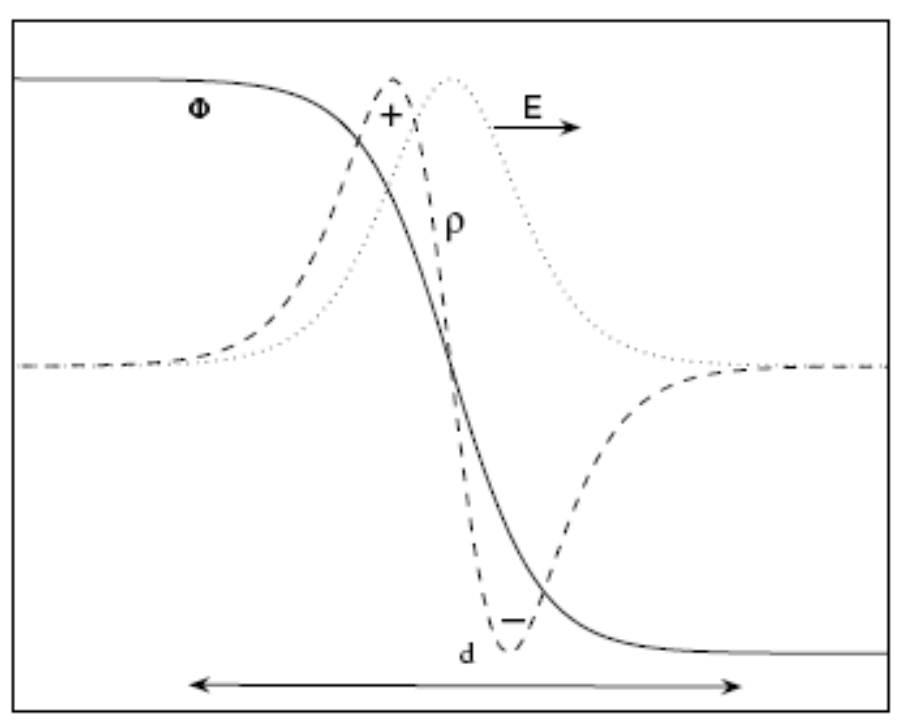

Figure 2.5. One dimensional profiles of a weak electric double layer potential (full line), space charge density (dashed line), and electric field (dotted line) [28].

\subsection{Electric Double Layers in Multi-Component Plasma}

Historically, laboratory EDL experiments have been performed in ionized gases of a single ion species. The physics of EDL formation is sufficiently complicated that a single ion species is preferred for ease of comparing experimental results to theory. However, space plasmas are generally composed multiple ionized species. Therefore, EDLs in space must include many ion populations. The dependence of space EDLs on the abundances of minor ions is an open and interesting question. A related issue is how the initial ion abundances are reflected in the relative abundances of the accelerated ions. For an EDL in a partially ionized plasma, the accelerated particles will consist of only those particles in the plasma that were ionized. However, the relative abundances of the accelerated ions will be the same as the relative abundances of the source plasma only if all the ions in the plasma flow into the EDL with the same characteristic speed.

To obtain the essential equations that describe a multi-ion component EDL, we begin with the simple case of a steady state one-dimensional EDL that supports a potential drop $\phi_{D L}$. Some of the plasma particles are accelerated through the potential drop of the EDL, while others are reflected back into the bulk plasma. Inside the EDL, the positive and negative particles are accelerated in opposite directions by the electric 


\section{Chapter 2: Plasma Sheaths and Double Layers}

field generated by the opposite space charges. At the edges of the layer, $z=0$ and $z=d(d$ is the thickness of the layer), quasineutrality requires that the electric field vanishes. For trapped Maxwellian electrons and ions and monoenergetic beams of ions and electrons entering from high potential side and low potential sides of the EDL, respectively, the Sagdeev potential has the form

$$
\begin{aligned}
&-V(\phi)= n_{e 0} e^{-e\left(\phi_{D L}-\phi\right) / k_{B} T_{e}}+\frac{m i_{e}}{e}\left(v_{e 0}^{2}+\frac{2 e \phi}{m}\right)^{1 / 2}+ \\
& \sum_{j}\left[n_{j 0} e^{-e \phi / k_{B} T_{j}}+\frac{M_{j} i_{j}}{e}\left(v_{j 0}^{2}+\frac{2 e\left(\phi_{D L}-\phi\right)}{M_{j}}\right)^{1 / 2}\right],
\end{aligned}
$$

where $i_{e}$ and $i_{j}$ are the electron and ion beam currents, respectively. Since the electric field is zero at the layer boundaries, where $V(0)=V\left(\phi_{D L}\right)$, the generalized current condition for a strong, multi-component EDL is

$$
i_{e}=\sum_{j} i_{j}\left(\frac{M_{j}}{m}\right)^{1 / 2} .
$$

For the particular case of an EDL consisting of only electrons and one singly charged positive ion species, Eq. (2.30) reduces to $i_{e} / i_{i}=(M / m)^{1 / 2}$; commonly known as the Langmuir condition [27].

Even without a complete solution of the EDL, key information about the physics of the multi-ion species EDL can still be obtained from the boundary conditions, specifically Eq. (2.27). Unlike the case of a single ion species plasma, where equality is satisfied by the ion species reaching its own sound speed, for multiple ion species the equality may be satisfied by ion speeds faster (supersonic) or slower (subsonic) than the corresponding ion sound speed. The two simplest solutions of Eq. (2.27) at the edge of the EDL are for all ions reaching the same speed, the system Bohm speed $-c_{\text {system }}$ (equal to the abundance weighted average of the sound speeds of the constituents ions), or for each species attaining its individual Bohm speed at the edge of the EDL. This result is the same as was obtained for the two ion species sheath case described previously.

As will be described in Chapter 5, laser induced fluorescence measurements of $\mathrm{Ar}^{+}$ion velocity distribution functions on the low and high potential sides of a helicon plasma EDL have been performed in two gas mixtures, argon-helium and argon-xenon to 


\section{Chapter 2: Plasma Sheaths and Double Layers}

determine which solution best describes the ion speeds at the edge of a real, multi-ion species EDL. These gas mixtures are amenable to LIF diagnosis while also having large differences in the individual ion sound speeds. For an Ar-He mixture, argon is more easily ionized (15.76 eV ionization threshold for argon versus $24.59 \mathrm{eV}$ for helium) and therefore the argon ions are expected to dominate the ion density. The $\mathrm{Ar}^{+}$sound speed is more than three times smaller than the $\mathrm{He}^{+}$sound speed (atomic mass of $40 \mathrm{amu}$ for $\mathrm{Ar}$ versus $4 \mathrm{amu}$ for helium). For the same reasons, $\mathrm{Ar}^{+}$is non-dominant and plays the role of the lighter ion (131 amu and $12.13 \mathrm{eV}$ ionization energy for xenon) in the Ar-Xe mixture. Defining $\eta$ and $(1-\eta)$ as the ratios of the argon ion and helium ion densities relative to the electron density for Ar-He mixture, the inequality of Eq. (2.27) leads to critical values of $\eta$ and (1- $\eta)$ that depend on the argon and helium ions Mach numbers

$$
\begin{gathered}
1-\eta \geq \frac{\frac{1}{\mathrm{M}_{A r}^{2}}-1}{\frac{1}{\mathrm{M}_{A r}^{2}}-\frac{1}{\mathrm{M}_{H e}^{2}}} \\
\eta \leq \frac{1-\frac{1}{\mathrm{M}_{H e}^{2}}}{\frac{1}{\mathrm{M}_{A r}^{2}}-\frac{1}{\mathrm{M}_{H e}^{2}}}
\end{gathered}
$$

Solving this coupled inequality system leads to the solution shown graphically in Figure 2.6. Over the range of $\mathrm{Ar}^{+}$and $\mathrm{He}^{+}$Mach numbers from 0 to 2, there are an infinity of solutions that require one species enters the EDL at a supersonic speed while the other species enters at a subsonic speed (the light gray areas in Figure 2.6). The hatched areas in Figure 2.6 correspond to negative values of $\eta$ in Eq. (2.31) and therefore have no physical meaning. The dark gray areas correspond to the values of $\eta$ greater than 1 and are also not solutions of Eq. (2.31).The consequences of this approach on the formation of an EDL in multi-component plasma are the following: since electrons are purely Maxwellian species, electron density must follow the double layer potential, while the densities of ion species must be enhanced or depleted depending of their initial velocities. 


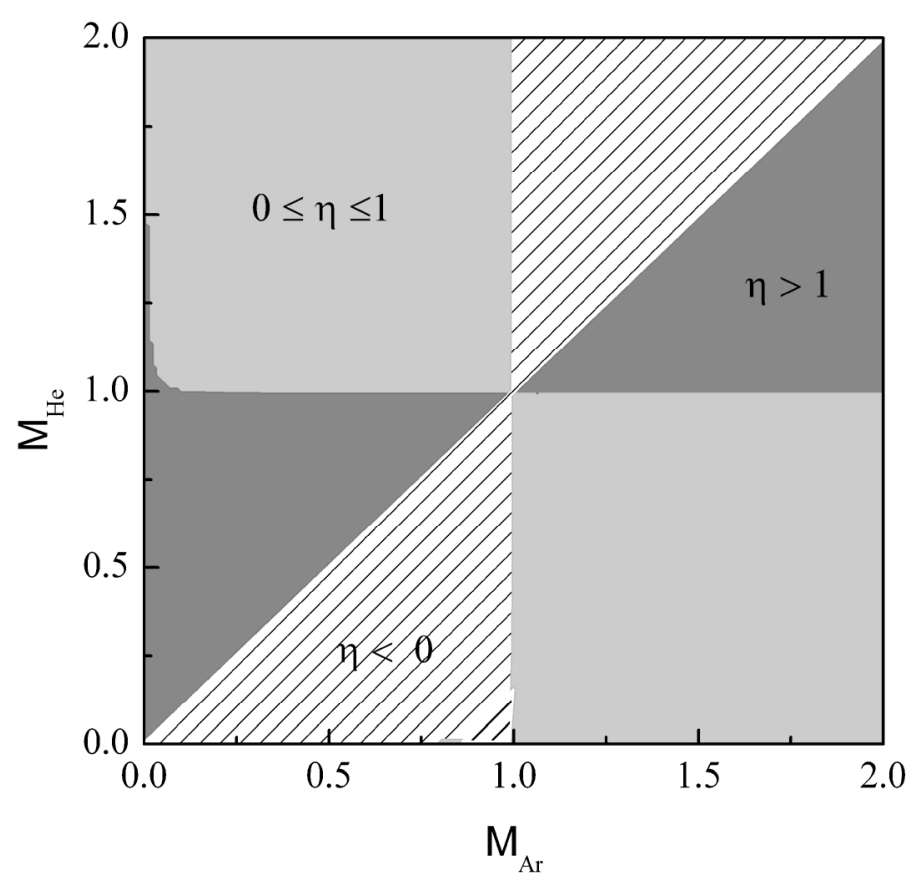

Figure 2.6. $\mathrm{Ar}^{+}$relative density $\eta$ versus $\mathrm{Ar}^{+}$and $\mathrm{He}^{+}$Mach numbers (Eqs. 2.31 (a) and (b)). The light gray areas are the only solutions of inequalities (2.31) for which $0 \leq \eta \leq 1$, i. e., one species entering the EDL is supersonic and the other is subsonic. The hatched and dark grey areas are non-physical solutions of Eqs. 2.31 for which the relative fractional density is negative or bigger than 1 , respectively. 


\section{Chapter 2 References}

${ }^{1}$ R. N. Franklin, J. Phys. D: Appl. Phys. 36, R309 (2003)

${ }^{2}$ I. Langmuir, Gen. Elec. Rev. XXVI, 731 (1923)

${ }^{3}$ I. Langmuir, Proc. Natl. Acad. Sci. XIV, 627 (1928)

${ }^{4}$ D. Bohm, The Characteristics of Electrical Discharges in Magnetic Fields, eds A. Guthrie and R. K. Wakerling, NY McGraw-Hill, chap. 3, page 77 (1949)

${ }^{5}$ N. Sternberg and V. Godyak, IEEE Trans. Plasma Sci. 31, 665 (2003)

${ }^{6}$ K. U. Riemann, IEEE Trans. Plasma Sci. 32, 2265 (2004)

${ }^{7}$ N. Sternberg and V. Godyak, IEEE Trans. Plasma Sci. 32, 2271 (2004)

${ }^{8}$ M. A. Lieberman and A. J. Lichtenberg, Principles of plasma discharges and material processing, John Wiley \& Sons, New York (1994)

${ }^{9}$ K. U. Riemann, J. Phys. D: Appl. Phys. 24, 493 (1991)

${ }^{10}$ K. Köhler, J. W. Coburn, D. E. Horne, E. Kay, and J. H. Keller, J. Appl. Phys. 57, 59 (1985)

${ }^{11}$ M. A. Lieberman, IEEE Trans. Plasma Sci. 16, 638 (1988)

${ }^{12}$ V. Godyak and N. Sternberg, Phys. Rev. A 42, 2299 (1990)

${ }^{13}$ M. Benilov, IEEE Trans. Plasma Sci. 31, 678 (2003)

${ }^{14}$ R. Chodura, Phys. Fluids, 251628 (1982)

${ }^{15}$ R. N. Franklin, J. Phys. D: Appl. Phys. 36, 34 (2003)

${ }^{16}$ K. U. Riemann, IEEE Trans. Plasma Sci. 23, 709 (1995)

${ }^{17}$ W. Lennartsson, Planet Space Sci. 28, 135 (1980)

${ }^{18}$ B. Hultqvist, Planet. Space Sci. 19, 749 (1971)

${ }^{19}$ C. G. Falthammar, Invited lecture-Second Latin American Conference on Space Geophysics, July 8-11, Cuernavaca, Mexico (1991)

${ }^{20}$ L. Anderson, R. E. Ergun, D. L. Newman, J. P. McFadden, C. W. Carlson, and Y. J. Yu, Phys. Plasmas 9, $3600(2002)$

${ }^{21}$ R. L. Stenzel, M. Ooyama, and Y. Nakamura, Phys.Fluids. 24, 708 (1981)

${ }^{22}$ D. Andersson, J. Phys. D: Appl. Phys. 14, 1403 (1981)

${ }^{23}$ R. Hatakeyama, Y. Suzuki, and N. Sato, Phys. Rev.Lett. 50, 1203 (1983)

${ }^{24}$ G. Hairapetian and R. L. Stenzel, Phys. Fluids B 3, 899 (1991)

${ }^{25}$ N. Sadeghi, T. Nakano, D. J. Trevor, and R. A. Gottscho, J. Appl. Phys. 70, 2552 (1991)

${ }^{26}$ R. Plamondon, J. Telchmann, and S. Torven, J. Phys. D: Appl. Phys. 21, 286 (1988)

${ }^{27}$ M. A. Raadu, Phys. Rep. 178, 27 (1989)

${ }^{28}$ C. Charles, Plasma Sources Sci. Technol. 16, R1 (2007)

${ }^{29}$ M. A. Raadu and J. J. Rasmussen, Astrophys. and Space Sci. 144, 43 (1988)

${ }^{30}$ L. P. Block, Astrophys. and Space Sci. 55, 59 (1978)

${ }^{31}$ P. Coakley, N. Hershkowitz, R. Hubbard, and G. Joyce, Phys. Rev. Lett. 40, 230 (1980) 
${ }^{32}$ L. P. Block, Astrophys. Space Sci. 55, 59 (1978)

${ }^{33}$ R. Z. Sagdeev, Rev. Plasma Phys. 23, 91 (1966)

${ }^{34}$ F. Verheest and M. A. Hellberg, J. Plasma Physics 57, 465 (1997) 


\section{Chapter 3: The Physics of Helicon Plasma Sources}

\subsection{Introduction to Helicon Plasma Sources}

In the 1960's, helicon waves were first investigated in solid state plasmas ${ }^{1}$ and in gaseous plasmas. ${ }^{2}$ The basic theory of these waves was extensively studied in that period by Woods, ${ }^{3}$ Klozenberg et al. ${ }^{4}$ and Davies et al. ${ }^{5}$ The generation of high plasma density in the helicon discharge was first investigated by Boswell. ${ }^{6}$ In the 1980's, the interest in helicon plasma sources was renewed by the emergence of their use as gas laser media, plasma reactors for material processing, and plasma lenses for high energy particle beams.

Helicon discharges are sustained by electromagnetic waves propagating in magnetized plasma in the so-called helicon modes. The driving frequency in these discharges is typically in the radio-frequency range of 1 to $50 \mathrm{MHz}$ (the industrially licensed radio-frequency of $13.56 \mathrm{MHz}$ is commonly used for material processing discharges). It is interesting to note that, in contrast to other rf discharges (capacitive and inductively coupled), helicon discharges are considered to be wave heated even though they operate in the rf range. Because the phase velocity of electromagnetic waves in magnetized plasma can be much lower than the speed of light, the helicon wavelength is comparable to the discharge system size even at radio-frequencies much smaller than the standard rf frequency of $13.56 \mathrm{MHz}$.

The magnetic field in helicon discharges varies from 20 to $2000 \mathrm{G}$ and magnetic field strengths of $\sim 1000 \mathrm{G}$ are often employed for fundamental plasma studies employing helicon sources. Excitation of the helicon wave is provided by an $\mathrm{rf}$ antenna that couples to the transverse mode structure of the wave across an insulating chamber wall. The electromagnetic wave mode propagates along the plasma column and the wave energy is transferred to electrons through collisional or collisionless damping mechanisms. Resonant coupling of the helicon mode to the antenna can lead to discontinuous changes in the plasma density for small changes in the source parameters. This phenomenon, known as a "mode jump", restricts the operating regime for a given helicon source

design. In a series of experiments, Boswell and co-workers ${ }^{7,8,9,10}$ investigated the 
structure and propagation of helicon waves excited in the 7-10 MHz range. They showed that on-axis peak densities of $\sim 10^{13} \mathrm{~cm}^{-3}$ (in argon) could be created in a 10-cm diameter tube with only $1 \mathrm{~kW}$ of rf power, a $1 \mathrm{kG}$ of magnetic field strength, and for a variety of $\mathrm{rf}$ antenna configurations.

\subsection{Operational Principles of Helicon Plasma Sources}

Helicon waves belong to the general class of plasma waves known as whistler waves which are right-hand circularly polarized electromagnetic waves in free space. Helicon waves differ from classical whistler waves in two main aspects: 1) they propagate at frequencies close to the low frequency limit of the electron cyclotron wave, so that electron inertia effects are small, and 2) they are modes of bounded systems, therefore their purely electromagnetic character cannot be maintained.

Since the helicons are propagating "whistler" wave modes in an axially magnetized, finite diameter plasma column, the electric and magnetic fields of the helicon modes have radial, axial, and, usually azimuthal variations. They propagate in high plasma densities at relatively low magnetic fields and their operating frequency, $\omega$, relative to key plasma frequencies is characterized by:

$$
\omega_{L H} \ll \omega \ll \omega_{c e}, \omega_{p e}^{2} \gg \omega \omega_{c e},
$$

where, $\omega_{L H}$ is the lower hybrid frequency, $\omega_{p e}=\sqrt{n e^{2} / \varepsilon_{0} m}$ is the electron plasma frequency, $\omega_{c e}=e B / m_{e}$ is the electron cyclotron frequency, and $n, e, \varepsilon_{0}, m$, and $B$ are the plasma density, elementary charge, dielectric permittivity of the vacuum, electron mass, and the uniform background magnetic field strength, respectively. Including ion inertia effects, the lower hybrid frequency is given by

$$
\frac{1}{\omega_{L H}^{2}}=\frac{1}{\omega_{p i}^{2}+\omega_{c i}^{2}}+\frac{1}{\omega_{c e} \omega_{c i}}
$$

where $\omega_{p i}=\sqrt{n Z^{2} e^{2} / \varepsilon_{0} M}$ and $\omega_{c i}=Z e B / M$ (with $Z e$ and $M$ the ion charge and mass, respectively) are the ion plasma and ion cyclotron frequencies. In high density plasmas, the first term is negligible and 


$$
\omega_{L H} \cong \sqrt{\omega_{c e} \omega_{c i}} .
$$

In the recent years the physical processes responsible for efficient helicon source operation (high plasma densities given the rf input power) have been extensively studied over a wide variety of operating regimes. Collisional processes, ${ }^{11,12}$ Landau damping, ${ }^{13,14}$ helicon wave penetration, ${ }^{15}$ antenna localized acceleration, ${ }^{16,17}$ mode conversion near the lower hybrid frequency, ${ }^{18}$ nonlinear trapping of fast electrons, ${ }^{19,20}$ and ion heating ${ }^{21}$ have all been considered.

Recent studies of helicon plasma sources have focused on both high efficiency operation and strong wave damping, neither of which is fully explained by either collisional or Landau damping processes. The possible role of a population of fast electrons, constituting a non-Maxwellian component of the electron distribution function, in ionizing the background gas in a helicon source is another important and related issue.

Through the helicon wave dispersion relation, plasma density and parallel wave number obey a fixed relationship for a given magnetic field strength ${ }^{22}$

$$
k=\sqrt{k_{\perp}^{2}+k_{\|}^{2}} \approx \frac{\omega}{k_{\|}} \cdot \frac{\omega_{p}^{2}}{\omega_{c e} e^{2}}=e \mu_{0} v_{p}\left(\frac{n}{B}\right),
$$

where $k$ is the wave number, $\mu_{0}$ the magnetic permeability in vacuum, and $v_{p}=\omega / k_{\|}$is the helicon wave's phase velocity along the tube. Generally $k_{\perp}$ is fixed by the tube radius $\mathrm{J}_{1}\left(k_{\perp} a\right)=0$ (where $\mathrm{J}_{1}$ is the Bessel function of first kind). It follows then that for a gas which has a peak in the ionization cross-section at $\sim 50 \mathrm{eV}$, such as argon, matching the wave phase speed to electrons with kinetic energies at the peak of the ionization cross section requires

$$
\frac{B}{n}=220 a, v_{p}=f \lambda=4.19 \times 10^{6} \mathrm{~ms}^{-1}, \text { and } f=\frac{32}{a}
$$

with $n$ in units of $10^{13} \mathrm{~cm}^{-3}$, the tube radius $a$ is in $\mathrm{cm}$, and the driving frequency $f$ is in $\mathrm{MHz}$. For the usual industrial frequency of $13.56 \mathrm{MHz}$, a tube of $\sim 5 \mathrm{~cm}$ diameter will require a magnetic field of $\sim 275 \mathrm{G}$ to generate a plasma density of $5 \times 10^{12} \mathrm{~cm}^{-3}$. When these design targets are met in practice, the density performance is generally consistent with this simple prediction. 
Even in the case of uniform magnetic fields, the mechanisms of plasma creation and loss in helicon discharges are not completely understood. The axial profile of plasma density downstream of the antenna is sensitive to many factors such as neutral pressure, antenna design, and magnetic field strength. In the particular case of small diameter tubes, the axial density scale length may be much shorter than the calculated damping distance and may be related to depletion of neutrals or formation of double layers. ${ }^{23}$

\subsection{WVU Helicon Plasma Source}

\subsubsection{Helicon Source and the Diffusion Chamber}

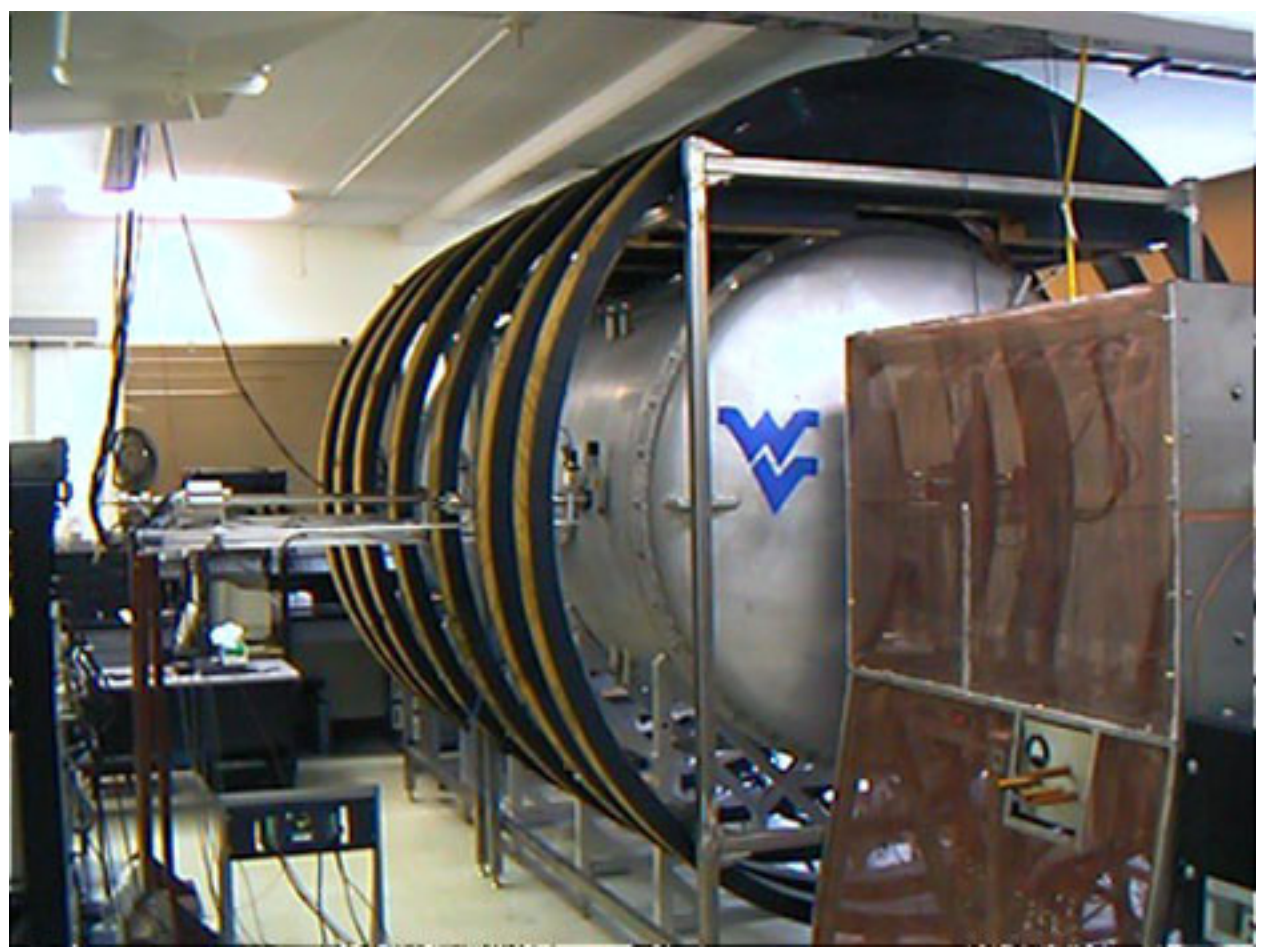

Figure 3.1. HELIX (foreground) and LEIA (large aluminum chamber) system. HELIX is surrounded by a Faraday cage for $\mathrm{rf}$ shielding. The large electromagnets surrounding LEIA are roughly $3 \mathrm{~m}$ in diameter. ${ }^{24}$

The helicon plasma source used for investigations in the present work consists of two distinct parts: the HELIX (Hot hELIcon eXperiment) source where high density plasma is created, and a large expansion chamber, LEIA (Large Experiment on Instabilities and Anisotropies) into which the plasma flows from HELIX. The HELIX 
helicon plasma source and the LEIA diffusion chamber are shown in Figures 3.1 and 3.2. HELIX consists of a $61 \mathrm{~cm}$ long, $10 \mathrm{~cm}$ diameter Pyrex tube mated coaxially with a 91 $\mathrm{cm}$ long, $15 \mathrm{~cm}$ diameter stainless steel tube.

An rf amplifier able to deliver up to $2 \mathrm{~kW}$ over a frequency range of $6-18 \mathrm{MHz}$ is used to generate the helicon plasma. The source can operate in a continuous wave mode or pulsed mode. Switching between continuous wave mode and pulsed mode, as well as changing the pulse duty cycle, is accomplished with a pulse generator that amplitude modulates the rf signal before the amplification stage. The plasma produced in the source expands into a $4 \mathrm{~m}$ long, $2 \mathrm{~m}$ diameter aluminum diffusion chamber - LEIA. The LEIA expansion chamber is surrounded by seven electromagnets which provide an axial magnetic field of $0-140 \mathrm{G}$.

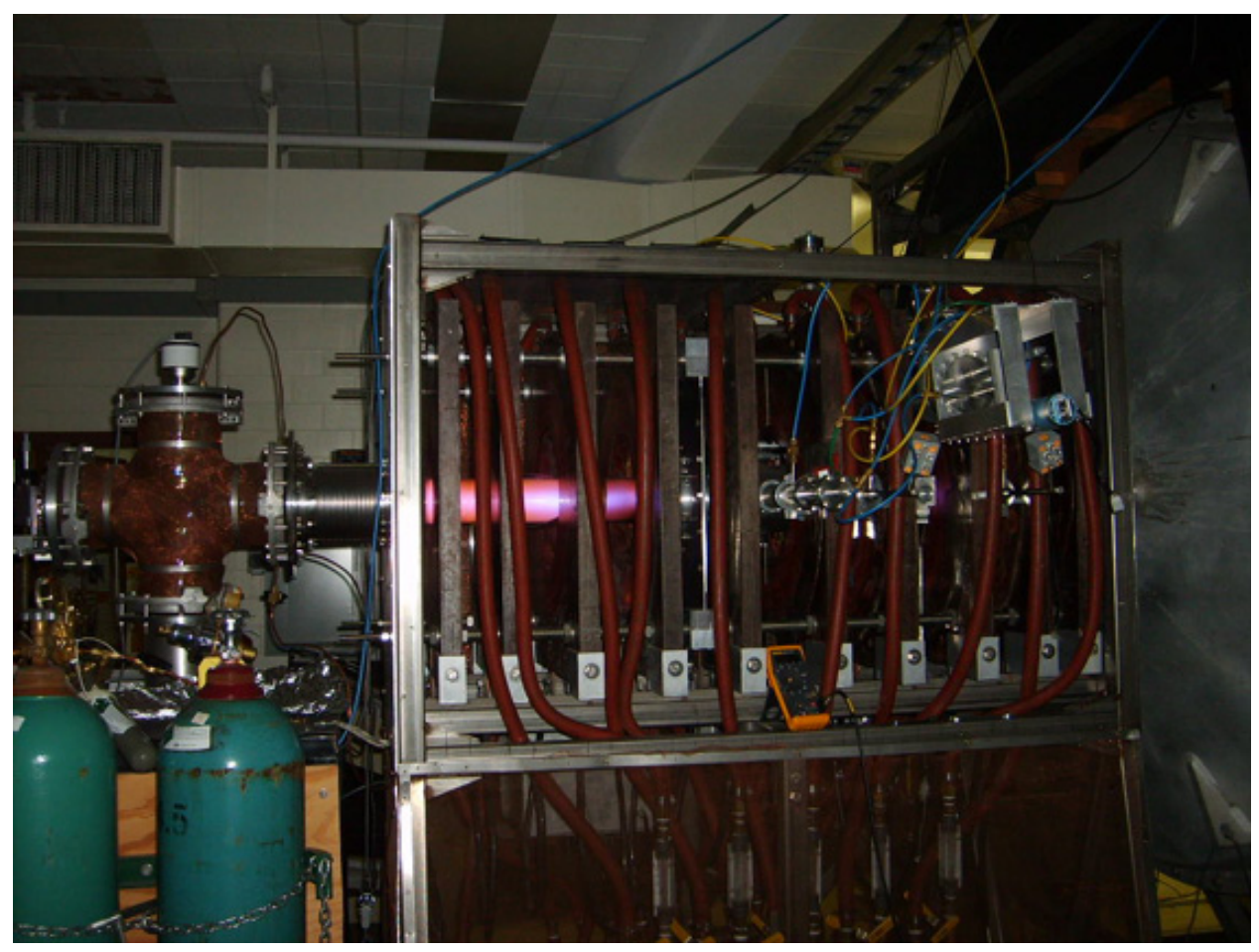

Figure 3.2. The HELIX helicon plasma source. On the far left is the mating flange with one of the pumping stations. Ten electromagnets are used to axially confine the plasma. The antenna is tightly wrapped around the tube at about $37 \mathrm{~cm}$ from the closed end. 


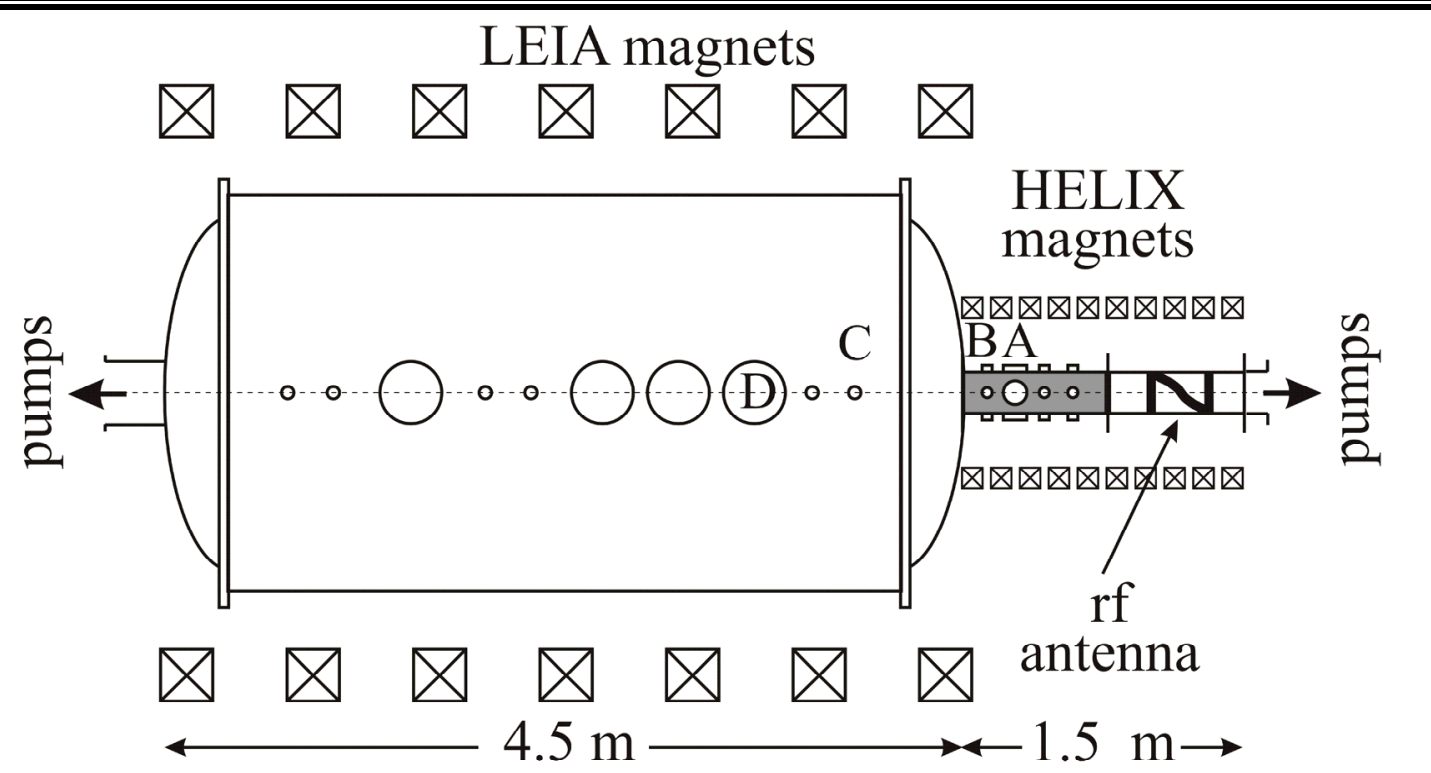

Figure 3.3. HELIX-LEIA schematic with diagnostic locations A, B, C, and D labeled.

\subsubsection{Vacuum System}

The HELIX-LEIA system is pumped differentially and the gas inlet valve is near the rf antenna. The HELIX pumping station consists of a Pfeiffer TMU 520 turbomolecular pump backed by a Pfeiffer MD 4T diaphragm roughing pump. LEIA is pumped by two Pfeiffer TMU 1600 turbomolecular pumps with Pfeiffer MD-8 membrane backing pumps, both located at the end of LEIA opposite of HELIX. Each LEIA pump can be run at a rotation frequency of $400 \mathrm{~Hz}$ or $600 \mathrm{~Hz}$, allowing for three pumping rates in the expansion chamber. There is a slight pressure gradient along the source axis, while in the expansion chamber the pressure is nearly constant and almost one order of magnitude smaller than the pressure in HELIX. The gas (or gas mixture) flow rate is controlled by two MKS 1179 mass flow calibrated valves with a PR-4000 flow controller. The flow controller regulates the flow of argon up to $200 \mathrm{sccm}$ with an accuracy of $1 \%$, and the flow of helium or xenon up to $20 \mathrm{sccm}$ with an accuracy of $0.1 \%$. 


\subsubsection{The Magnetic Field System}

Ten water cooled solenoids (see Figure 3.2) create the axial magnetic field in HELIX. Two Xantrex XFR de power supplies connected in parallel provide up to $400 \mathrm{~A}$ current for the electromagnets. The maximum attainable magnetic field is $1200 \mathrm{G}$. Seven 9' diameter water cooled electromagnets confine the plasma in the downstream chamber (Figure 3.4 (a)). Each electromagnet is made of 20 turns of water cooled, 0.36 " $\times 0.41$ " hollow rectangular aluminum tubing. Current of up to $200 \mathrm{Amp}$ is provided by an EMI dc power supply and the maximum achievable axial magnetic field in LEIA is $140 \mathrm{G}$. Under typical operating conditions, in the connection region between the helicon source and the expansion chamber, there is an axial magnetic field gradient of nearly $10 \mathrm{G} / \mathrm{cm}$ over a distance of $70 \mathrm{~cm}$. Figure 3.4 (b) shows the on-axis magnetic field strength and its gradient in the HELIX-LEIA combined system as calculated with a two-dimensional numerical model that was validated with measurements along the system axis. The evolution of contour lines of constant magnetic flux (flux tubes) are shown in Figure 3.4 (c) for a constant magnetic field strength in HELIX of $B_{H}=600 \mathrm{G}$ and for two magnetic field strengths in LEIA: $70 \mathrm{G}$ (dash-dot line); and $14 \mathrm{G}$ (solid line). 
LEIA magnets

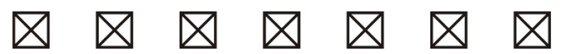
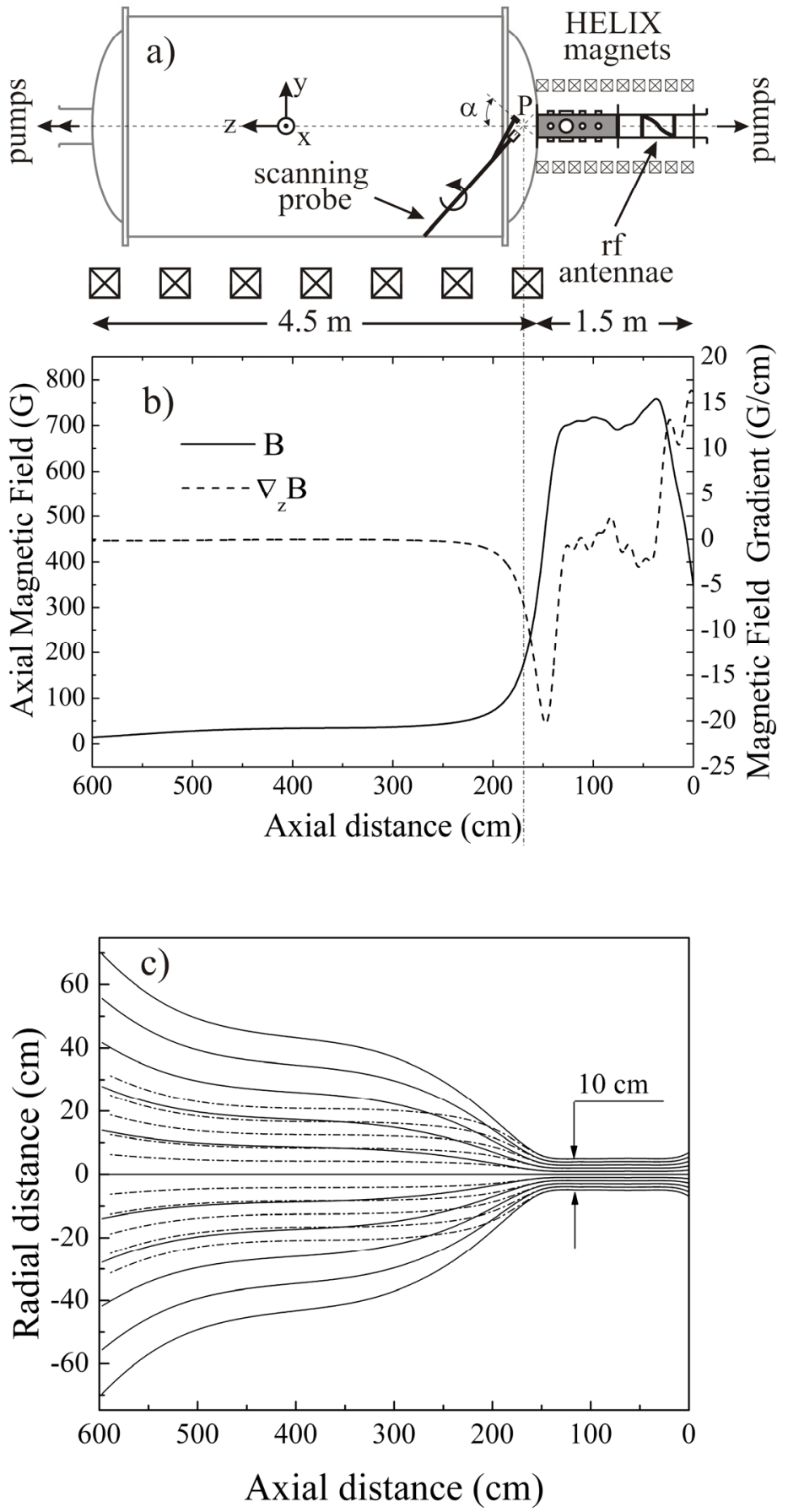

Figure 3.4. a) HELIX-LEIA system. b) Magnetic field profile and magnitude, and magnetic field gradient versus axial position over the entire length of HELIX-LEIA system. c) Contours of constant magnetic flux showing the increased divergence that results when the magnetic field in the expansion region decreases from $70 \mathrm{G}$ (dash-dot line) to $14 \mathrm{G}$ (solid line) for a constant source field of $600 \mathrm{G}$. 


\subsubsection{Rf Generator, Matching Network, and Antenna}

The rf power used for plasma generation in HELIX is supplied by a $50 \mathrm{MHz}$ Wavetek model-80 function generator followed by a $30 \mathrm{~dB}$ ENI $2000 \mathrm{rf}$ amplifier. The rf power is transmitted from the amplifier to the source through a high frequency coaxial cable and a $\pi$-type matching network to match the $50 \Omega$ output impedance of the amplifier to the antenna/matching network system. The matching network consists of one large, tunable, Jennings high voltage vacuum capacitor with a range $20-2000 \mathrm{pF}$ range (the "load" capacitor) and three smaller "tuning" Jennings high voltage capacitors (two with a range of 4-250 $\mathrm{pF}$ and one with a range of 5-500 pF). The three tuning capacitors are in parallel with each other and the combination is in series with the load capacitor.

A $19 \mathrm{~cm}$ long, half wave, $m=+1$, helical antenna couples the rf energy into the plasma (Figure 3.5). The antenna is tightly wrapped around the Pyrex tube at about $37 \mathrm{~cm}$ axial location from the closed end of HELIX. A detailed description of the matching network and the antenna can be found in the dissertations of Balkey ${ }^{25}$ and Sun. ${ }^{26}$

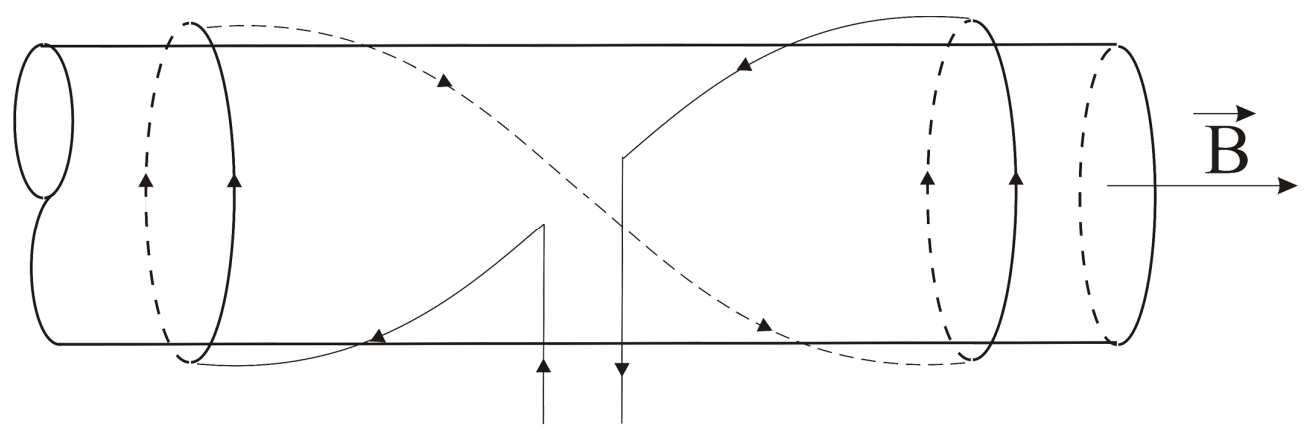

Figure 3.5. Schematic of $m=+1$ helical antenna ${ }^{27}$ used in these experiments.

\subsubsection{Typical plasma parameters in HELIX-LEIA}

The typical plasma (Ar, Xe, and He) parameters in HELIX and LEIA are shown in Table 3.1. For typical HELIX-LEIA operating conditions, the plasma is collisional in the source (HELIX) and collisionless in the expansion chamber (LEIA). 
Table 3.1. Typical plasma parameters in HELIX and LEIA

\begin{tabular}{|c|c|c|}
\hline Parameter & Helicon source & Expansion chamber \\
\hline$n_{e}\left(10^{12} \mathrm{~cm}^{-3}\right)$ & $0.1-10$ & $0.001-0.1$ \\
\hline$B(\mathbf{G})$ & $500-1200$ & $5-70$ \\
\hline$p_{n}$ (mTorr) & $1.5-10$ & $0.15-1$ \\
\hline$L(\mathrm{~cm})$ & 150 & 450 \\
\hline$T_{e}(\mathrm{eV})$ & $4-12$ & $2-8$ \\
\hline$T_{i}(\mathrm{eV})$ & $<1$ & $<1$ \\
\hline$\lambda_{D}(\mathrm{~cm})$ & $5 \times 10^{-3}-8 \times 10^{-4}$ & $3 \times 10^{-2}-6.5 \times 10^{-3}$ \\
\hline$r_{e}(\mathbf{c m})$ & $4 \times 10^{-3}-2 \times 10^{-2}$ & $5 \times 10^{-2}-1.5$ \\
\hline \multirow{3}{*}{$r_{i}(\mathbf{c m})$} & Ar: $2 \times 10^{-1}-6 \times 10^{-1}$ & Ar: $4-60$ \\
\hline & Xe: $4 \times 10^{-1}-1$ & Xe: $8-105$ \\
\hline & He: $8 \times 10^{-2}-2 \times 10^{-1}$ & He: $1.5-18$ \\
\hline$\omega_{p e}\left(10^{10} \mathrm{rad} / \mathrm{s}\right)$ & $1.8-18$ & $0.18-1.8$ \\
\hline \multirow{2}{*}{$\omega_{c e}\left(10^{9} \mathrm{rad} / \mathrm{s}\right)$} & Ar: $5.2-34$ & Ar: 52 - 340 \\
\hline & Xe: $1.25-8.2$ & Xe: $12.5-82$ \\
\hline \multirow{3}{*}{$\omega_{c i}\left(10^{6} \mathrm{rad} / \mathrm{s}\right)$} & Ar: $0.12-0.3$ & Ar: $1.2 \times 10^{-3}-1.6 \times 10^{-2}$ \\
\hline & Xe: $0.04-0.09$ & Xe: $4 \times 10^{-4}-5 \times 10^{-3}$ \\
\hline & He: $1.2-3$ & He: $1.2 \times 10^{-2}-1.6 \times 10^{-1}$ \\
\hline
\end{tabular}




\section{Chapter 3 References}

${ }^{1}$ R. Bowers, C. Legendy and F. E. Rose, Phys. Rev.Lett. 7, 339 (1961)

${ }^{2}$ G. N. Harding and P. C. Thonemann, Proc. Phys. Soc. 85, 317 (1965)

${ }^{3}$ L. C. Woods, J. Fluid Mech. 18, 401 (1964)

${ }^{4}$ J. P. Klozenberg, B. McNamara and P. C. Thonemann, J. Fluid Mech. 21, 545 (1965)

${ }^{5}$ B. J. Davies and P. J. Christiansen, Plasma Phys. 11, 987 (1969)

${ }^{6}$ R. W. Boswell, Plasma Phys. Controlled Fusion, 26, 1147 (1970)

${ }^{7}$ R. W. Boswell, R. K. Porteus, A. Prytz, A. Bouchoule and P. Ranson, Phys. Lett. A 91, 163 (1982)

${ }^{8}$ R. W. Boswell and D. Henry, Appl. Phys. Lett. 47, 1095 (1985)

${ }^{9}$ R. W. Boswell and R. K. Porteus, Appl. Phys. Lett. 50, 1130 (1987)

${ }^{10}$ R. W. Boswell and R. K. Porteus, J. Appl. Phys. 62, 3123 (1987)

${ }^{11}$ R. W. Boswell and F. F. Chen, IEEE Trans. Plasma Sci. 25, 1229 (1997)

${ }^{12}$ F. F. Chen and R. W. Boswell, IEEE Trans. Plasma Sci. 25, 1245 (1997)

${ }^{13}$ Y. Mouzouris and J. E. Scharer, Phys. Plasmas 5, 4253 (1998)

${ }^{14}$ F. F. Chen and D. D. Blackwell, Phys. Rev. Lett. 82, 2677 (1999)

${ }^{15}$ R. Kinder and M. J. Kushner, J. Vac. Sci. Technol. A 19, 76 (2001)

${ }^{16}$ G. G. Borg, J. Bright, and I. V. Kamenski, Plasma Phys. Controlled Fusion, 40, 987 (1998)

${ }^{17}$ A. W. Degeling and R. W. Boswell, Phys. Plasmas 4, 2748 (1997)

${ }^{18}$ S. Cho and J. G. Kwak, Phys. Plasmas 4, 4167 (1997)

${ }^{19}$ R. T. S. Chen and N. Hershkowitz, Phys. Rev. Lett. 80, 4677 (1998)

${ }^{20}$ A. Degeling, N. Mikhelson, R. Boswell, and N. Sadeghi, Phys. Plasmas 5, 572 (1998)

${ }^{21}$ M. M. Balkey, R. F. Boivin, J. L. Kline, and E. E. Scime, Plasma Sources Sci.Technol. 10, 284 (2001)

${ }^{22}$ F. F. Chen, Plasma Phys. Controlled Fusion 33, 339 (1991)

${ }^{23}$ C. Charles, Plasma Sources Sci.Technol. 18, R1 (2007)

${ }^{24}$ P. Keiter, PhD Dissertation, West Virginia University (1999)

${ }^{25}$ M. Balkey, PhD Dissertation, West Virginia University (2000)

${ }^{26} \mathrm{X}$. Sun, PhD Dissertation, West Virginia University (2005)

${ }^{27}$ M. Light and F. F. Chen, Phys. Plasmas 2, 1086 (1995) 


\section{Chapter 4: Plasma Diagnostics}

It is convenient to divide diagnostic techniques into three general categories: $e x$ situ, in situ-intrusive, and in situ-non-intrusive. The common characteristic of ex situ techniques is that they sample an aliquot of the plasma reactor's contents and transfer it elsewhere for examination. On the other hand, the division of in situ techniques into intrusive and non-intrusive is somewhat arbitrary. To some extent, any diagnostic technique perturbs the plasma. In some cases this perturbation may be so slight as to be negligible, while in others it may be quite considerable. One example of an in situ diagnostic which would generally be called intrusive is a Langmuir probe. In this case, a physical object is inserted into the plasma, thereby perturbing it. However, depending upon the information desired, this perturbation may or may not be negligible.

\subsection{Langmuir Probe}

Electrostatic probes are undisputedly the oldest and most widely used diagnostic tools in plasma physics. The technical description and first theoretical explanation of the electrostatic probe was developed by Langmuir; ${ }^{1}$ hence it is widely known as the Langmuir probe. In its simplest form, a Langmuir probe is a conducting wire inserted in the plasma and the current versus applied voltage, $V$, characteristic, I-V, is measured with respect to a reference electrode. The plasma parameters which can easily be obtained using measured current-voltage characteristic and the relevant probe theory are the electron temperature $T_{e}$, the electron density $n_{e}$, the floating potential $V_{f}$, and plasma potential $V_{p}$ (or space potential $V_{S}$ as shown in Figure 4.1). There are five regions of interest in the idealized Langmuir probe trace shown in Figure 4.1. For applied potentials more positive than the space potential (the potential of the plasma), $V_{p}$, all the available electrons in the vicinity of the probe are collected by the probe, i.e., electron saturation. The collected current in this regime can increase if the sheath expands with increasing applied potential. The space potential is identifiable by the "knee" in the I-V curve. At the far left of the curve, the probe is in ion saturation, $I_{i, \text { sat }}$, all electrons are repelled, and all available ions are collected. The floating potential $V_{f}$ is the value of the applied 
potential for which the collected ion and electron currents are equal. The exponential part of the curve (in a Maxwellian plasma) is called the transition region. In the transition region, the ion current is negligible and the electrons are partially repelled by the negative potential difference, $V-V_{p}$, between the plasma potential and the applied potential.

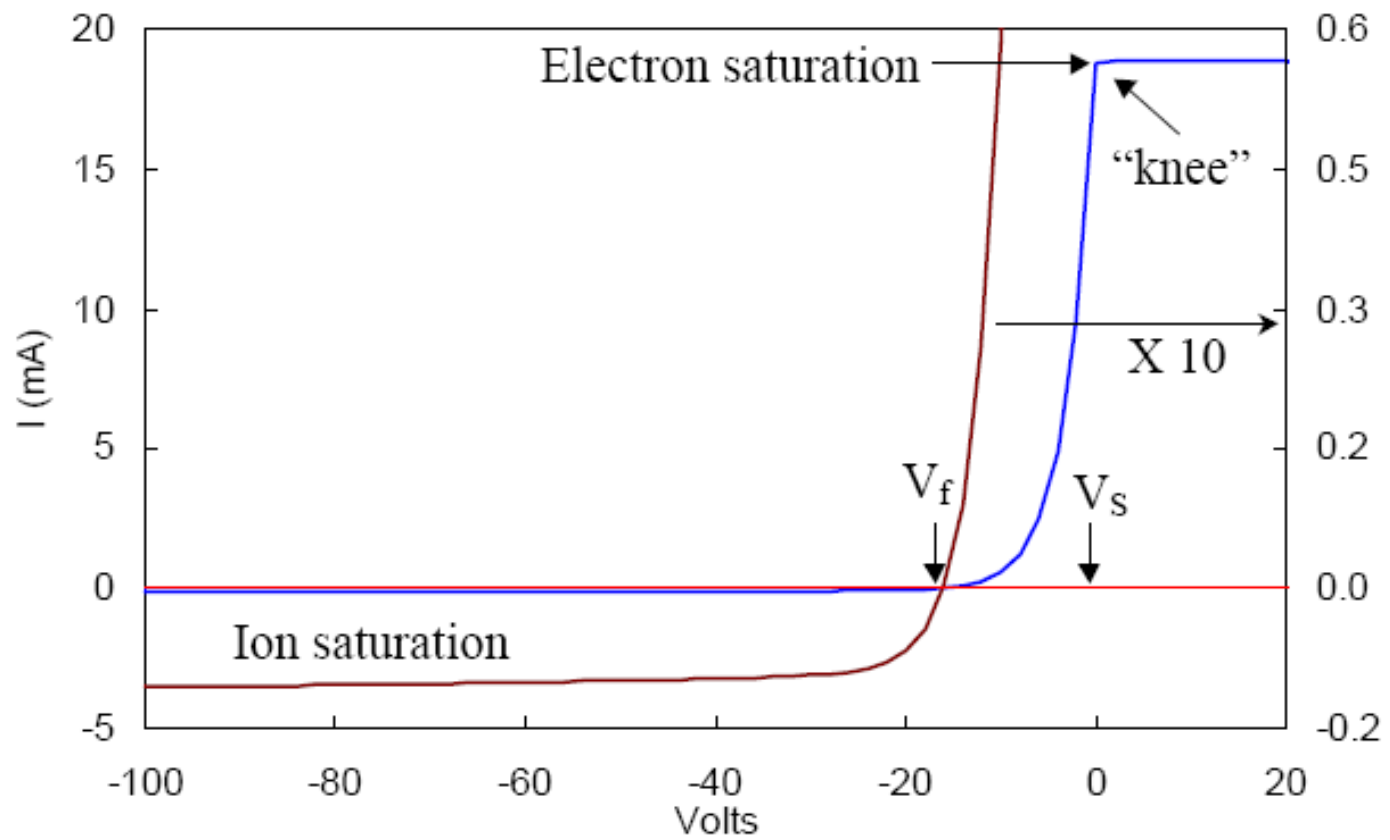

Figure 4.1. An idealized Langmuir I-V curve. The red curve has been expanded 10 times to emphasize the ion current. ${ }^{2}$

In a magnetized rf plasma, collisions, the fluctuating plasma potential, and the magnetic field all affect a Langmuir probe I-V characteristic. Magnetic fields strong enough to make the electron Larmor radius smaller than the probe radius limit $I_{e, s a t}$ to only few tens of $I_{i, s a t}$ because in saturation the probe depletes the charge particles only on magnetic field lines that it intercepts. The larger ion gyroradius provides a larger effective ion collection area. Additional electrons can be collected only if they diffuse across the magnetic field. The "knee" in this case is indistinct and the I-V curve (Figure 4.2) is exponential only over a range of few $k_{B} T_{e}$ above the floating potential. For the weak magnetic fields in LEIA $(\sim 10 \mathrm{G})$ the electrons are unmagnetized $\left(r_{c e} \cong 5 \mathrm{~mm}>r_{p}=0.5\right.$ $\mathrm{mm}$ ) and the magnetic field effects can be ignored. For HELIX, magnetic fields $\sim 700 \mathrm{G}$, the electron cyclotron radius $\left(r_{c e} \sim 70 \mu \mathrm{m}\right)$ is much smaller than the probe radius and magnetic effects must be included in the analysis. According to Lafambroise and 
Rubinstein, ${ }^{3}$ even in strong magnetic fields the electron phase-space density or velocity distribution function can still be described by the usual Maxwell-Boltzmann distribution with an additional correction term for a hypothetical potential well arising from the presence of the magnetic field. Since the electron distribution is not distorted but only shifted in energy, measurements obtained by applying the unmagnetized probe theory to the data still provide an accurate assessment of changes in density, potential, and electron temperature in magnetized plasmas.

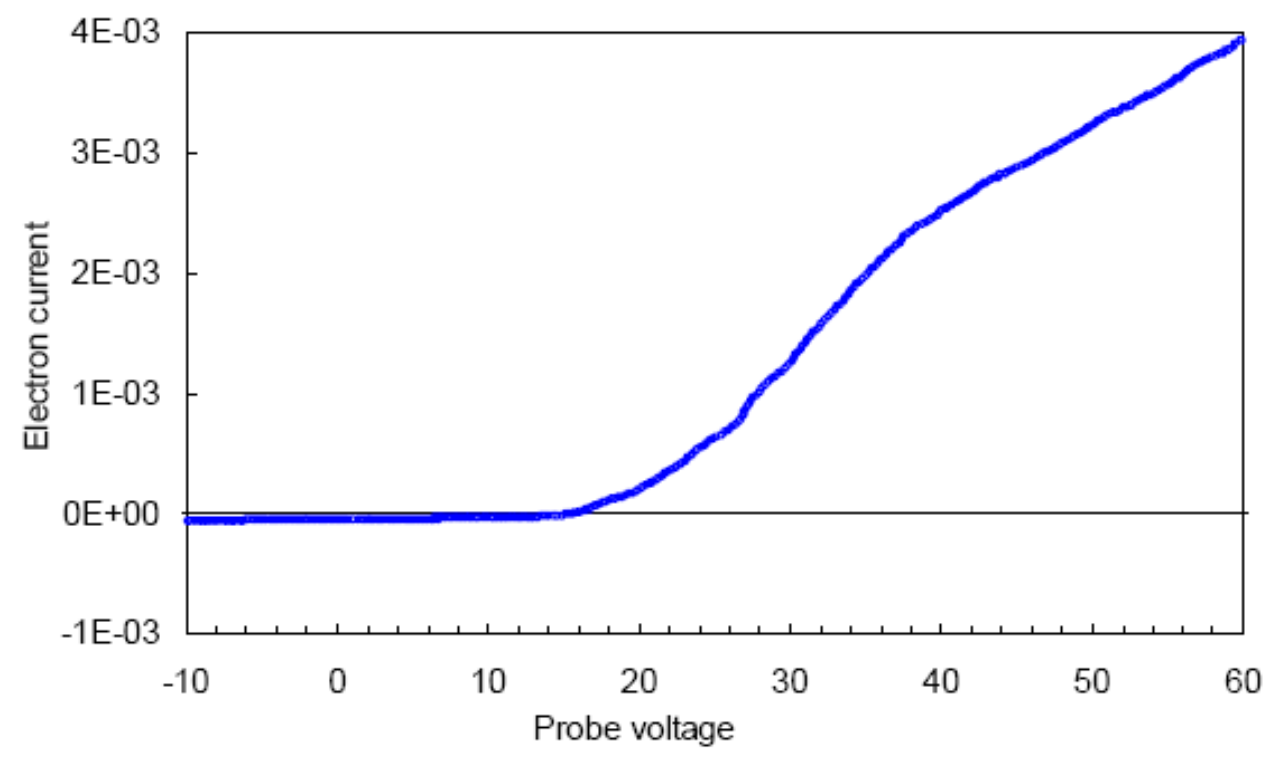

Figure 4.2. I-V curve typical of a magnetized, rf plasma $^{2}$

For a simple, unmagnetized, collisionless, Maxwellian plasma comprised of one electron population and two positive ions species, the collected current for applied voltages around the floating potential is approximately ${ }^{4}$ :

$$
\begin{aligned}
& I\left(V-V_{p}\right)= \\
& e A_{p}\left(n_{i 1} \sqrt{\frac{k_{B} T_{e}}{M_{1}}}+n_{i 2} \sqrt{\frac{k_{B} T_{e}}{M_{2}}}\right)\left(\frac{n_{e}}{n_{i 1} \sqrt{\frac{2 \pi m}{M_{1}}}+n_{i 2} \sqrt{\frac{2 \pi m}{M_{2}}}} e^{e\left(V-V_{p}\right) / k_{B} T_{e}}-\frac{A_{S}}{A_{p}} e^{-1 / 2}\right),
\end{aligned}
$$

where $m$ and $M_{j}, j=1,2$ are electron and ion masses respectively, $n_{e}$ and $n_{i j}$ are electron and ions densities, $A_{p}$ is the probe area and $A_{S}$ the sheath area. At high plasma densities (greater than $10^{11} \mathrm{~cm}^{-3}$ ), the sheath around a negatively biased probe is so thin that the 
surface area of the sheath is essentially the same as the surface area of the probe, $A_{S}=A_{p}$. Equivalently, the ratio of probe radius to the Debye length $\left(\lambda_{D}=\left(\varepsilon_{0} k_{B} T_{e} / n_{e} e^{2}\right)^{1 / 2}\right)$ is larger than 1. Even with the added complication of more than one ion species, Eq. (4.1) can be used to determine the electron temperature $T_{e}$ and plasma potential $V_{p}$. Note that the electron temperature for any plasma is only defined if the electrons distribution is Maxwellian. In non-Maxwellian plasmas, the concept of a temperature is valid only for a specific portion of the distribution function. For a pure Maxwellian electron distribution function, the electron temperature is obtained from the slope of $\ln \left(I_{e}\right)$ versus the applied voltage, i.e., beginning with

$$
I_{e}\left(V-V_{p}\right)=e n_{e} A_{p} \sqrt{\frac{k_{B} T_{e}}{2 \pi m}} e^{e\left(V-V_{p}\right) / k_{B} T_{e}}
$$

and taking the natural logarithm,

$$
\ln \left(I_{e}\right)=\frac{e V}{k_{B} T_{e}}+\text { const }
$$

Eq. (4.3) yields straight line with a slope $\Delta \ln \left(I_{e}\right) / \Delta V \propto 1 / T_{e}$ on a semi-logarithmic plot. The electron temperature $k_{B} T_{e} / e$ in electron volts is given by

$$
k_{B} T_{e}=e\left[\frac{\Delta \ln \left(I_{e}\right)}{\Delta V}\right]^{-1} .
$$

Any deviation from linearity on the semi-logarithmic plot indicates a deviation of the electron energy distribution function from a pure Maxwellian. In such a case, the electron population cannot be described by a single electron temperature.

In contrast to a single ion species plasma, for a two ion species plasma the electron density cannot be uniquely determined from a measurement of electron temperature and the ion saturation current,

$$
I_{i, s a t}=-0.61 e n_{e} A_{p}\left(\frac{n_{i 1}}{n_{e}} \sqrt{\frac{k_{B} T_{e}}{M_{1}}}+\frac{n_{i 2}}{n_{e}} \sqrt{\frac{k_{B} T_{e}}{M_{2}}}\right) .
$$

The ion saturation current contains two unknowns, $n_{i l}$ or $n_{i 2}$, which can be related to the electron density through the quasineutrality relation $\left(n_{i 1}+n_{i 2} \cong n_{e}\right)$. A recent two-ion species fluid model of Bai and co-workers ${ }^{5}$ demonstrated that normalized ion densities are related to electron density through the ionization rate constant of each species: 


$$
\begin{aligned}
\frac{n_{i 1}}{n_{e}} & =\frac{(1-\eta) k_{1}^{i z}}{k_{t}^{i z}} \\
\frac{n_{i 2}}{n_{e}} & =\frac{\eta k_{2}^{i z}}{k_{t}^{i z}}
\end{aligned}
$$

where $(1-\eta) / \eta$ is the partial pressure ratio (abundance ratio) of gas species 1 and 2, respectively; $k_{j}^{i z}$ is the ionization rate constant of each species,

$$
k_{j}^{i z}=\sigma_{j}^{i z}\left(\frac{8 k_{B} T_{e}}{\pi m}\right)^{1 / 2}\left(1+\frac{2 k_{B} T_{e}}{\varepsilon_{j}^{i z}}\right) e^{-\varepsilon_{j}^{i z} / k_{B} T_{e}},
$$

and $k_{t}^{i z}$ is the total ionization rate constant

$$
k_{t}^{i z}=(1-\eta) k_{1}^{i z}+\eta k_{2}^{i z} .
$$

In Eq. (4.7.a), $\sigma_{j}^{i z}$ is the ionization cross section of each species and $\varepsilon_{j}^{i z}$ is the ionization threshold energy of each species. By substituting the normalized ion densities into Eq. (4.5), the electron density can be determined from measurements of the ion saturation current and the electron temperature.

The floating potential $V_{f}$ is the potential to which an electrically isolated probe floats as a result of the difference in ion and electron mobilities. As noted previously, the plasma potential is the applied potential at which the current collection switches from partial repulsion of electrons to electron current saturation (the "knee" on Figure 4.1). An alternative approach to determining the plasma potential involves setting the applied potential equal to the floating potential, i. e. setting Eq. (4.1) equal to zero.

$$
\frac{e\left(V_{f}-V_{p}\right)}{k_{B} T_{e}}=\ln \left(\frac{n_{i 1}}{n_{e}} \sqrt{\frac{2 \pi m}{M_{1}}}+\frac{n_{i 2}}{n_{e}} \sqrt{\frac{2 \pi m}{M_{2}}}\right)-\frac{1}{2}
$$

Although complicated, Bai's method eliminates the issue of the unknown relative ion densities and can provide an accurate determination of the plasma potential:

$$
V_{p}=V_{f}+\frac{k_{B} T_{e}}{e}\left\{\frac{1}{2}-\ln \left[\frac{(1-\eta) k_{1}^{i z}}{k_{t}^{i z}} \sqrt{\frac{2 \pi m}{M_{1}}}+\frac{\eta k_{2}^{i z}}{k_{t}^{i z}} \sqrt{\frac{2 \pi m}{M_{2}}}\right]\right\} .
$$

For low temperature plasmas, Langmuir probes are typically fabricated from tungsten wires several millimeters in diameter. Because tungsten has a high melting point, the probe can be made small enough that perturbations are minimized. For high 


\section{Chapter 4: Plasma Diagnostics}

temperature plasmas, graphite probes with length and radii from 1 to $10 \mathrm{~mm}$ are typically used because graphite can withstand higher power loads. In magnetized plasmas, ideally the probe diameter should be a few times larger than the ion Larmor radius. In this work, both cylindrical and disc (planar), rf compensated, Langmuir probes were used. The cylindrical probe (Figure 4.3) consists of a $0.5 \mathrm{~mm}$ diameter graphite rod that extends 3 $\mathrm{mm}$ from an insulating alumina tube.

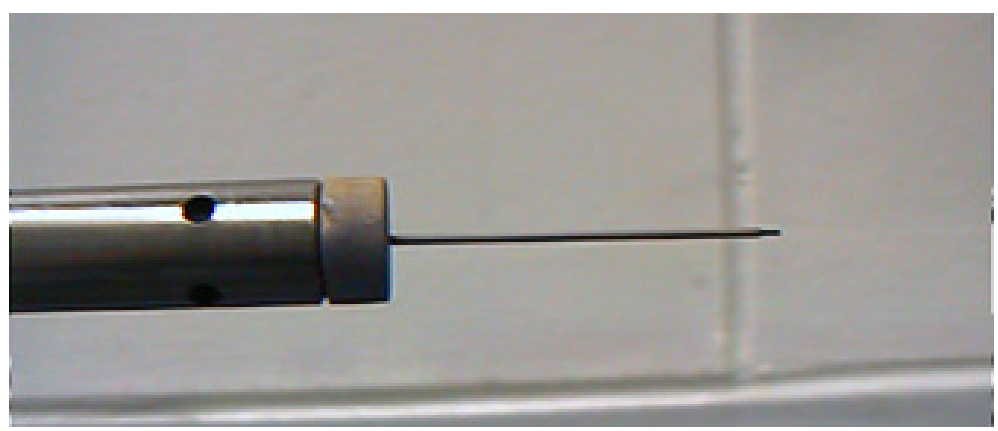

Figure 4.3. Cylindrical Langmuir probe

The planar probe (Figure 4.4) is fabricated from a tungsten sheet cut into a circle and spot welded to a tungsten rod. The diameter of the disc is $0.6 \mathrm{~cm}$. One side of the probe surface is coated with alumina powder to prevent current collection by that side of the disc. The tungsten rod, $2.5 \mathrm{~cm}$ long, is shielded from the plasma with an alumina tube. The probe is mounted on a shaft that can reach the junction between HELIX and LEIA and can translate along the symmetry axis of the chamber ( $z$ axis).

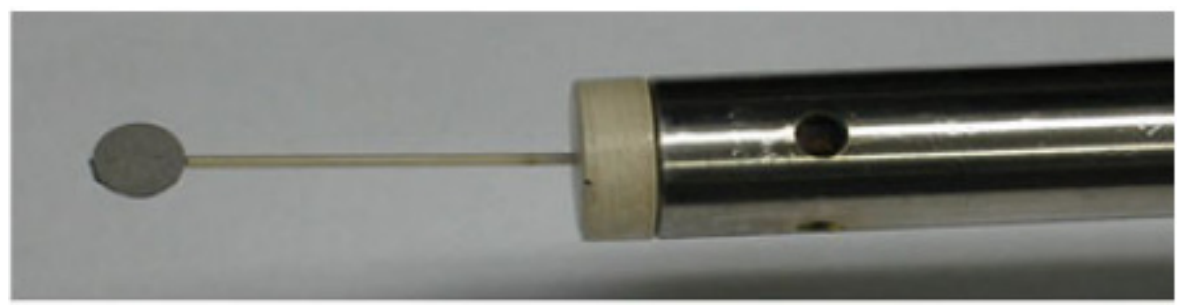

Figure 4.4. Planar Langmuir probe ${ }^{6}$

Both probes are rf compensated. The compensation is accomplished by a series of rf chokes covering the frequency range 6-18 MHz and a $10 \mathrm{nF}$ shorting capacitor. ${ }^{7}$ Although slightly more difficult to construct, the planar probe has the important advantage of being able to reach electron saturation in a high density, magnetized, helicon plasma (and therefore the planar Langmuir probe can directly measure the plasma 


\section{Chapter 4: Plasma Diagnostics}

potential). In high density magnetized plasmas, the collecting area of a cylindrical Langmuir probe increases with increasing positive bias voltage because the growing circularly symmetric sheath intercepts an ever increasing number of magnetic field lines. The sheath of the planar probe grows in the direction of the field and therefore the collecting area of the probe changes only modestly with increasing bias voltage (assuming the probe is inserted into the plasma with the normal of the disk parallel to the magnetic field direction).

\subsubsection{Druyvesteyn Differentiation Method for Obtaining the Electron Energy Distribution Function (EEDF)}

The classical Langmuir method for determination of the electron temperature, electron density and plasma potential assumes a Maxwellian electron energy distribution function (eedf), $f(\varepsilon)$. However, in low pressure discharges the eedf is generally nonMaxwellian and reported electron temperatures are actually an effective electron temperature $T_{\text {eff }}$ that corresponds to a mean electron energy $\langle\varepsilon\rangle$ determined from the eedf. Careful measurement of the transition region of the I-V characteristic can reveal details of the electron distribution if it is isotropic. Another method for determination of electron density $n_{e}$, the effective electron temperature $T_{e f f}$, and plasma potential $V_{p}$ directly from the eedf is the Druyvesteyn procedure. ${ }^{8}$ The Druyvesteyn technique consists of differentiating the probe characteristic to obtain the eedf and then determining $n_{e}$ and $T_{\text {eff }}$ from integrals of the eedf. The advantage of Druyvesteyn's method is that it can be used for non-Maxwellian plasmas in which the electron density is not simply related to a single electron temperature and the ion saturation current. The disadvantage of Druyvesteyn's method is that it employs the second derivative of the I-V curve for cylindrical Langmuir probes; a measurement that is strongly affected by noise. The signal-to-noise ratio can be improved by averaging many I-V measurements or by lowpass filtering the I-V measurement.

Perhaps even more important for the measurements presented in this work is that the classical Langmuir technique is not generally applicable for multi-ion species plasmas; especially when only the ion portion of the I-V characteristic is used. Because 


\section{Chapter 4: Plasma Diagnostics}

the velocity of the ions entering the sheath (the Bohm velocity) differs significantly from the single ion species case, use of the Langmuir analysis could lead to greatly exaggerated values for the electron density. Therefore, in this work, the Druyvesteyn method is used to calculate $n_{e}$ and $T_{\text {eff. }}$.

\subsubsection{Electron Energy Distribution Function from Planar Langmuir Probe Measurements}

For a planar probe aligned perpendicular to the plasma flow (the $z$ axis in the HELIX-LEIA system), the electron flux entering the sheath depends only on the $\mathrm{z}$ velocity component, $v_{z}$. The electron current collected by the probe is

$$
I_{e}=e n_{e} A_{p}\left\langle v_{z}\right\rangle,
$$

where $A_{p}$ is the probe area and $\left\langle v_{z}\right\rangle$ is the average electron velocity in $z$ direction. In terms of the one-dimensional electron velocity distribution function, $f\left(v_{z}\right)$,

$$
\left\langle v_{z}\right\rangle=n_{e}^{-1} \int_{v_{\min }}^{\infty} v_{z} f\left(v_{z}\right) d v_{z}
$$

where $v_{\min }=(-2 \mathrm{eV} / \mathrm{m})^{1 / 2}$ is the minimum velocity of an electron that can reach the probe for a bias voltage corresponding to the electron repelling region of the I-V characteristic. Taking the derivative of Eq. (4.10) and transforming from velocity to energy space in Eq. (4.11),

$$
f(\varepsilon)=\frac{m}{e^{2} A_{p}} \frac{d I_{e}}{d V} .
$$

Thus $f(\varepsilon)$ is the eedf and is obtained from the first derivative of the $\mathrm{I}-\mathrm{V}$ characteristic for a planar Langmuir probe. The electron density is determined directly from the normalization condition

$$
n_{e}=(2 m)^{-1 / 2} \int_{0}^{\infty} f(\varepsilon) \varepsilon^{-1 / 2} d \varepsilon
$$

and the effective electron temperature is related to the mean electron energy $\langle\varepsilon\rangle$ through

$$
T_{e f f}=\left(2 n_{e}\right)^{-1}(2 / m)^{1 / 2} \int_{0}^{\infty} f(\varepsilon) \varepsilon^{1 / 2} d \varepsilon
$$


A typical I-V characteristic obtained with the planar probe is shown in Figure 4.5. Also shown in Figure 4.5 is the first derivative of the I-V characteristic. Although the "knee" (the bend in the curve at the plasma potential) is less distinct than in the ideal I-V characteristic (Figure 4.1), the plasma potential $V_{p}$ is easily identified in the first derivative. Thus, for a planar probe, it is sufficient to use the first derivative of the I-V characteristic to obtain the eedf and then $n_{e}$ and $T_{e f f}$ are obtained from integration of the eedf.

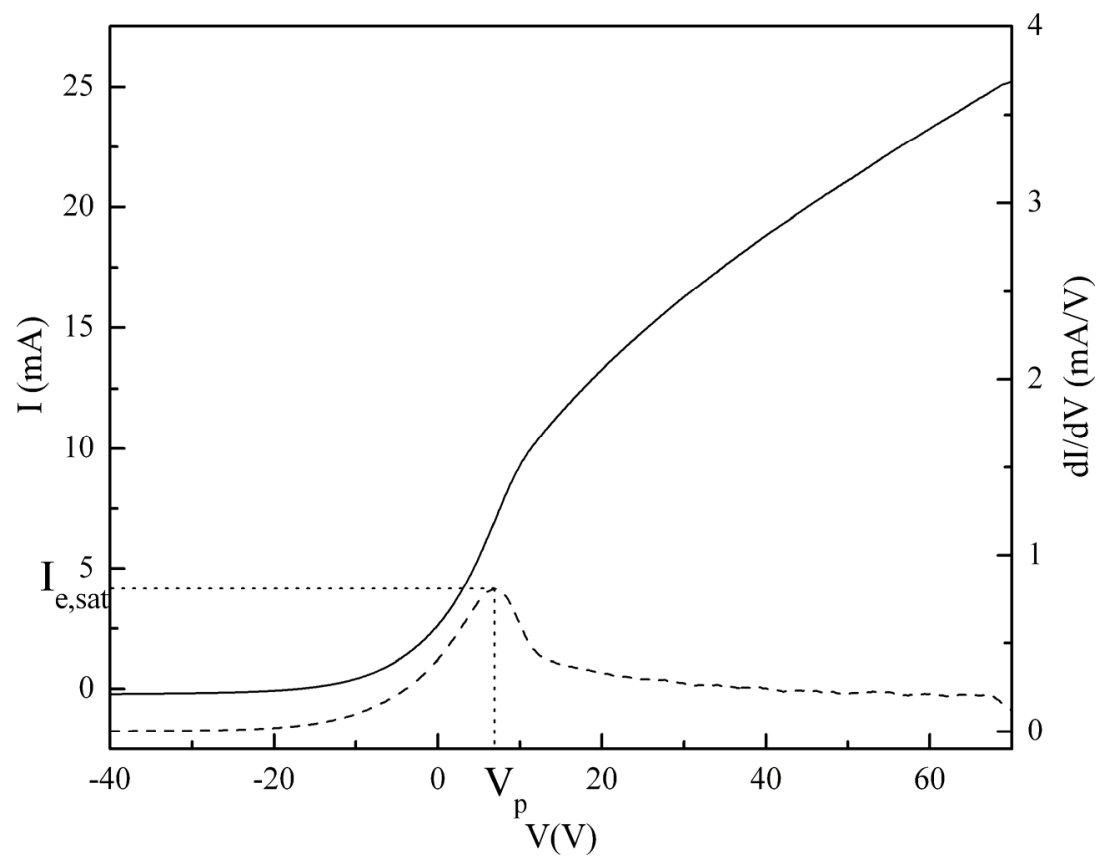

Figure 4.5. Typical planar probe I-V characteristic (solid line) and its first derivative (dash line)

\subsubsection{Electron Energy Distribution Function from Cylindrical Langmuir Probe Measurements}

Druyvesteyn [8] also demonstrated that his analysis method can be used for any non-concave probe surface. In the case of a cylindrical probe, the collected current is

$$
I_{e}=e A_{p} \pi \int_{v_{\min }}^{\infty} f\left(m v^{2} / 2-e V\right) v^{3} d v
$$

In terms of the electron energy $\varepsilon+e V=m v^{2} / 2$ 


$$
I_{e}=\frac{2 \pi e A_{p}}{m^{2}} \int_{0}^{\infty} f(v(\varepsilon))(\varepsilon+e V) d \varepsilon .
$$

Double differentiation of Eq. (4.16) with respect to the applied voltage $V$ gives the Druyvesteyn formula,

$$
f(v(\varepsilon))=\frac{m^{2}}{2 \pi e^{3} A_{p}} \frac{d^{2} I_{e}}{d V^{2}} .
$$

For an isotropic distribution, the evdf is transformed into the eedf through

$$
f(\varepsilon) d \varepsilon=4 \pi v^{2} f(v(\varepsilon)) d v .
$$

and Eq. (4.17) becomes

$$
f(\varepsilon)=\frac{2(2 m)^{1 / 2}}{e^{3} A_{p}} \varepsilon^{1 / 2} \frac{d^{2} I_{e}}{d V^{2}} .
$$

Thus, for a cylindrical Langmuir probe, the eedf is obtained from the second derivative of the collected current. The maximum of the first derivative or the zero crossing of the second derivative of the probe current with respect to the applied voltage is used to determine the plasma potential (see Figure 4.6). Determining the plasma potential by differentiating the $\mathrm{I}-\mathrm{V}$ characteristic is a more accurate process than visual inspection of a semilog plot of the electron current versus the probe bias voltage.
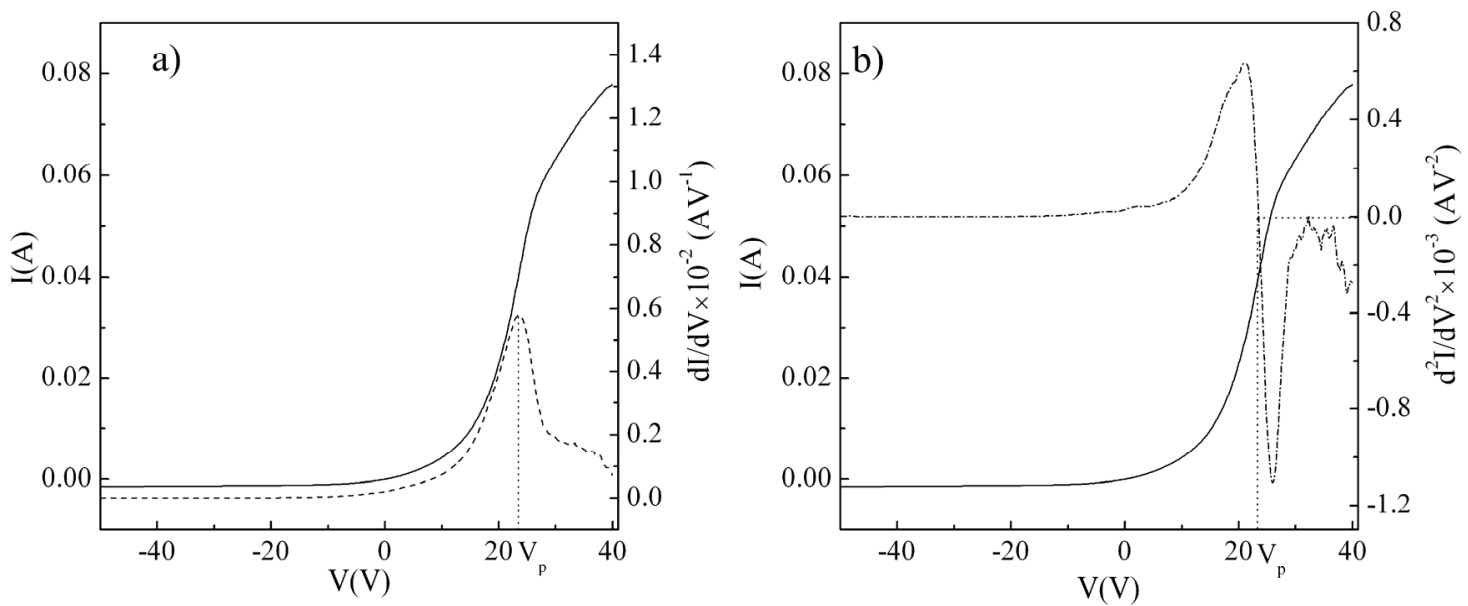

Figure 4.6. a) First (dashed line) and b) second derivative (dash-dot line) of a typical cylindrical Langmuir probe I-V characteristic (solid line). The plasma potential is the value on the abscissa where $d I / d V$ is a maximum, i.e., where $d^{2} I / d V^{2}$ is zero. 
In practice it is easier to work with another function called electron energy probability function (eepf), $F(\varepsilon)$. Mathematically, $F(\varepsilon)$, is not a "true" probability function, i.e. $\int_{-\infty}^{\infty} F(\varepsilon) d \varepsilon \neq 1$. The eepf is defined as

$$
F(\varepsilon)=\varepsilon^{-1 / 2} f(\varepsilon) .
$$

Eepfs are particularly effective in distinguishing between different kinds of energy distribution functions. Different discharge processes generate different and unique eepfs. For example, on the logarithmic plot shown in Figure 4.7, a Maxwellian eepf would be a straight line. ${ }^{9}$

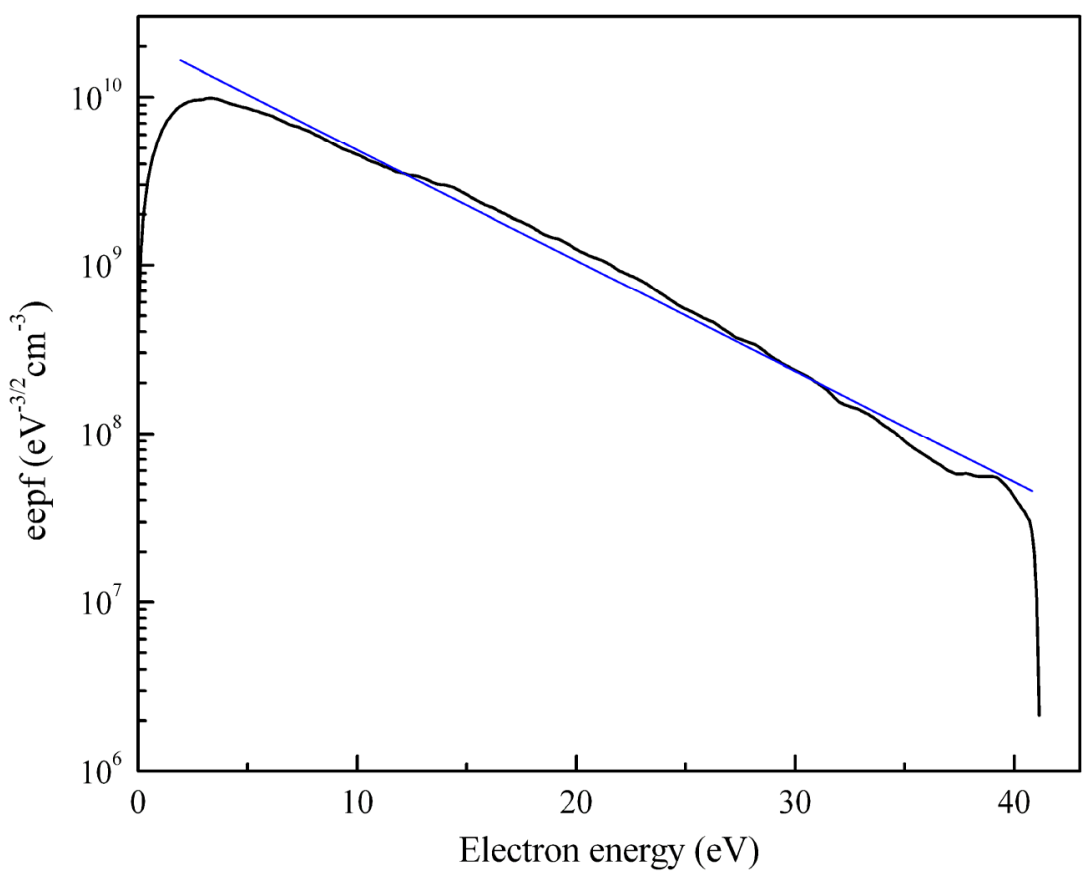

Figure 4.7. Electron energy probability function (eepf) for a quasi-Maxwellian distribution (data obtained in HELIX for a 1.3 mTorr Ar discharge). The electron temperature is the inverse of the eepf slope.

Substituting Eq. (4.19) in Eq. (4.20), the eepf can also be written in terms of the second derivative of the collected current.

$$
F(\varepsilon)=\frac{2(2 m)^{1 / 2}}{e^{3} A_{p}} \frac{d^{2} I_{e}}{d V^{2}} .
$$

Once the electron distribution function is known, the electron density is obtained by integrating the eedf over all possible energies 


$$
n_{e}=\int_{0}^{\infty} f(\varepsilon) d \varepsilon
$$

and the effective electron temperature is obtained from the weighted average of the electron energy $\langle\varepsilon\rangle$

$$
T_{e f f}=\frac{2}{3}\langle\varepsilon\rangle=\frac{2}{3 n_{e}} \int_{0}^{\infty} \varepsilon f(\varepsilon) d \varepsilon .
$$

It is worth repeating that concept of electron temperature for any plasma is only valid if the eedf is Maxwellian. For non-Maxwellian plasmas, a given electron temperature is valid only for a particular region of the distribution function. As noted by Godyak [9], the Debye length and ion current density in the Bohm-like expression of the ion current are governed by the so called screening temperature $T_{\text {screening }}$ and not by $T_{\text {eff }}$. The screening temperature is defined as

$$
T_{\text {screening }}=\frac{2}{n_{e}}\left(\int_{0}^{\infty} \varepsilon^{-1} f(\varepsilon) d \varepsilon\right)^{-1} \neq T_{\text {eff }} .
$$

For low pressure rf discharges, non-Maxwellian electron energy distributions, such as bi-Maxwellian and Druyvesteyn, are commonly observed. A bi-Maxwellian distribution has distinct populations of cold (low energy) and hot (high energy) electrons and can be represented by

$$
f(\varepsilon)=n_{e c} \frac{2}{\pi^{1 / 2}\left(k_{B} T_{e c}\right)^{3 / 2}} \varepsilon^{1 / 2} e^{-\varepsilon / k_{B} T_{e c}}+n_{e h} \frac{2}{\pi^{1 / 2}\left(k_{B} T_{e h}\right)^{3 / 2}} \varepsilon^{1 / 2} e^{-\varepsilon / k_{B} T_{e h}},
$$

where $n_{e c}$ and $n_{e h}$ are the densities of the cold and hot populations, respectively. The total density is the sum of the density components $\left(n_{e}=n_{e c}+n_{e h}\right)$. In this case, the screening temperature is given by

$$
\frac{T_{\text {screening }}}{n_{e}}=\left(\frac{n_{e c}}{T_{e c}}+\frac{n_{e h}}{T_{e h}}\right)^{-1} .
$$

A Druyvesteyn distribution is usually used to approximate an eedf that is depleted at high energies. The Druyvesteyn energy distribution includes more electrons close to the average energy and fewer electrons at higher energies than does a Maxwellian distribution. If elastic collisions dominate the electron energy losses and the electron 
mean free path $\lambda_{e n}$ is assumed to be constant, the general form of the Druyvesteyn distribution is

$$
f_{D}(\varepsilon)=A \varepsilon^{1 / 2} e^{-\frac{3 m}{m_{N}}\left(\frac{\varepsilon}{\Sigma_{0}}\right)^{2}},
$$

where $m$ and $m_{N}$ are the electron and neutral particle mass, respectively; $\Sigma_{0}=e \lambda_{e n} E$ is the energy gained by electrons over a mean free path $\lambda_{e n}$ along the electric field $E$; and $A=$ constant. The normalization condition (4.22) gives

$$
n_{e}=A \int_{0}^{\infty} \varepsilon^{1 / 2} e^{-\frac{3 m}{m_{N}}\left(\frac{\varepsilon}{\Sigma_{0}}\right)^{2}} d \varepsilon .
$$

After a change of variables in Eq. (4.28) and the use of gamma function $\left(\Gamma(\zeta) \equiv \int_{0}^{\infty} t^{\zeta-1} e^{-t} d t\right)$, the Druyvesteyn distribution function becomes:

$$
f_{D}(\varepsilon)=2 n_{e}[\Gamma(3 / 4)]^{-1}\left[\left(\frac{3 m}{m_{N}}\right)^{1 / 2} \Sigma_{0}^{-1}\right]^{3 / 2} \varepsilon^{1 / 2} e^{-\frac{3 m}{m_{N}}\left(\frac{\varepsilon}{\Sigma_{0}}\right)^{2}}
$$

As can be seen from Eq. (4.29) and Figure 4.8, the Druyvesteyn distribution decreases with energy more rapidly than the Maxwellian distribution with the same average energy.

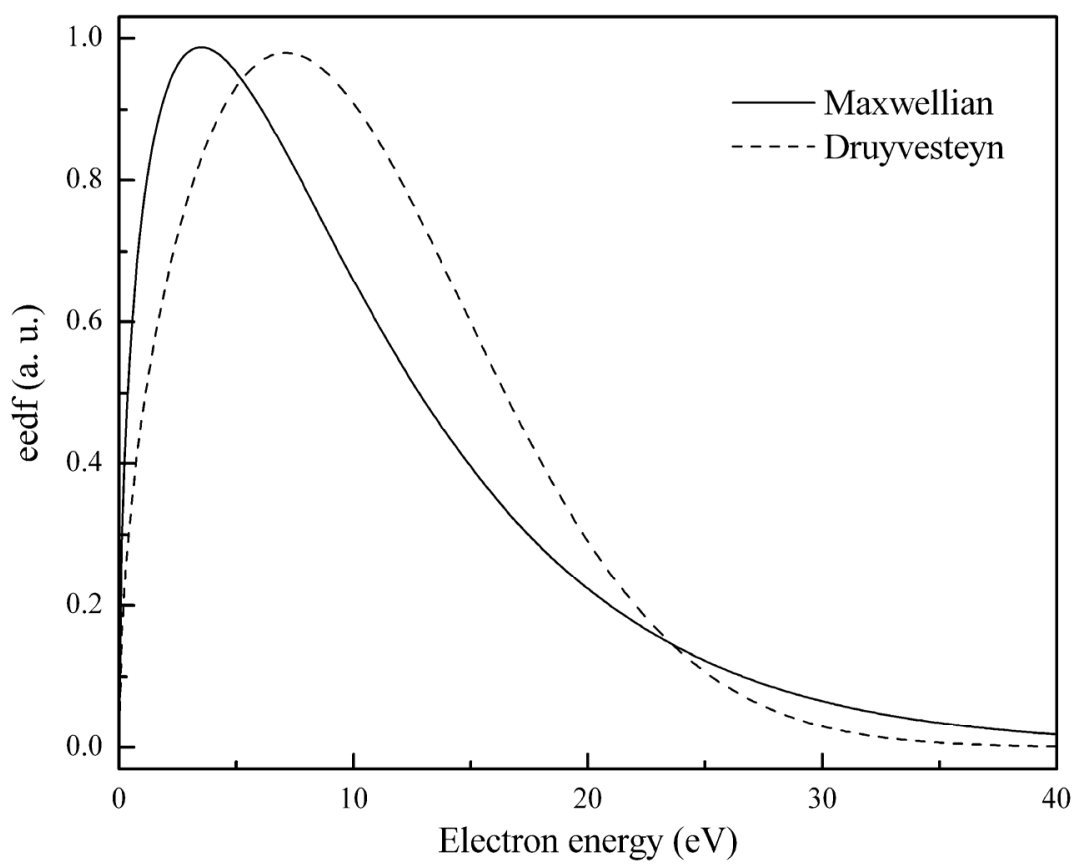

Figure 4.8. Maxwell and Druyvesteyn electron energy distributions for the same average electron energy of $10 \mathrm{eV}$. 
For a Dryuvesteyn distribution, the average electron energy is

$$
\langle\varepsilon\rangle=n_{e}^{-1} \int_{0}^{\infty} \varepsilon f_{D}(\varepsilon) d \varepsilon=\left(\frac{3 m}{m_{N}}\right)^{-1 / 2} e \lambda_{\mathrm{en}} E \frac{\Gamma(5 / 4)}{\Gamma(3 / 4)} .
$$

An important feature in Figure 4.8 is that both energy distributions are characterized by a high-energy tail. For an average electron energy of $10 \mathrm{eV}$, a significant number of electrons have energies above $25 \mathrm{eV}$. Thus, even for a modest value of the average energy there are enough electrons in the high-energy tail of the distribution to have a significant impact on the overall reaction rates in the plasma.

The full eedf can be recovered from the retarded electron component of the probe data (see Eq. 4.19). The challenge in using Eq. (4.19) to obtain electron energy distribution functions arises from performing two numerical differentiations of imperfect data without degrading the voltage resolution or amplifying the noise in the data. In this work, the probe data are digitally recorded and post-processed with finite difference and curve fitting algorithms to smooth and differentiate the signals. Oversampling and averaging over many realizations also helped to reduce noise levels in the measurements. Overly aggressive smoothing of the data was avoided so as to not wash out real features in the eedfs.

\subsection{Optical Emission Spectroscopy}

\subsubsection{Collisional Processes in Plasma: The Corona Model}

Creation of a quiescent helicon plasma requires specific choices for the magnetic field strength and profile, the rf frequency and power, and the gas flow into the chamber. As is true of all low temperature, quasi-neutral plasmas, a helicon plasma is a mixture of ions, electrons and neutrals that undergo collisions with each other. The collision frequency for a particular process is usually defined in terms of an average over all velocities v, assuming a Maxwellian distribution

$$
f_{\text {collision }}=n\langle\sigma v\rangle,
$$


where $n$ is the particle density and $\sigma$ the cross section for the collision process. All collisional processes can be divided into two classes: elastic and inelastic. Elastic collisions are those in which the internal energies of colliding particles do not change and the total kinetic energy is conserved. Inelastic collisions result in the transfer of energy from the kinetic energy of colliding partners into internal energy. The collisions in helicon plasmas can be further divided into two more categories: collisions directly related to ion production and charge exchange reactions between ions and neutrals.

\subsection{1.a. Electron Impact Excitation}

In electron impact excitation, an electron collides with an atom or ion and loses some of its energy to excitation of one of the bound electrons of the atom or ion. In a Corona model of excited state populations in a plasma, only impact excitation collisions from ground states are considered because the ground state population density is an order of magnitude larger than the population density of any of the excited levels. Cascade emission from a higher energy excited state into a given state (radiative gain from upper levels) is also neglected because of the very small population densities of the upper levels. However, the radiative de-excitation out of those higher energy states is the dominate loss mechanism out of excited states. Thus, a Corona model assumes that the excited levels are populated by electron impact excitation from the ground state and depopulated by spontaneous, radiative de-excitation.

The excitation process is represented by the general reaction

$$
A_{i}+e^{-} \rightarrow A_{j}+e^{-} .
$$

In this process, the free electron loses some of its kinetic energy to excitation of one of the bound electrons of the atom from the level $i$ (including the ground state) to a higher level $j$. The number of excitation collisions per unit time per unit volume is

$$
R=n_{e} n_{A}^{i}\left\langle v_{e} \sigma_{e-A}^{i-j}\right\rangle,
$$

where $n_{e}$ is the electron density, $n_{A}^{i}$ is the $i$-th level neutral density, $v_{e}$ is the electron velocity, and $\sigma_{e-A}^{i-j}$ is the cross section for electron excitation from level $i$ to level $j$. The 
average quantity in relation (4.32) is the electron impact rate coefficient. It can be easily calculated for a Maxwellian electron energy distribution function,

$$
f(\varepsilon)=\frac{2}{\pi^{1 / 2}\left(k_{B} T_{e}\right)^{3 / 2}} \varepsilon^{1 / 2} e^{-\varepsilon / k_{B} T_{e}} .
$$

The electron impact rate coefficients are given by the integral

$$
k_{i \rightarrow j}=\left\langle v_{e} \sigma_{e-A}^{i-j}\right\rangle=\sqrt{\frac{2}{m}} \int_{0}^{\infty} f(\varepsilon) \varepsilon^{1 / 2} \sigma_{e-A}^{i-j}(\varepsilon) d \varepsilon,
$$

where $m$ and $\varepsilon$ are the electron mass and energy respectively. In a similar fashion, the rate coefficient for excitation of an ion from a lower level $k$ to an upper level $m$ is

$$
k_{k \rightarrow m}=\left\langle v_{e} \sigma_{e-A^{+}}^{k-m}\right\rangle=\sqrt{\frac{2}{m}} \int_{0}^{\infty} f(\varepsilon) \varepsilon^{1 / 2} \sigma_{e-A^{+}}^{k-m}(\varepsilon) d \varepsilon .
$$

From the expressions above, it can be seen that the rates for electron impact excitation depend only on the electron temperature. To excite an atom or an ion from the ground state to a high energy level requires electrons with sufficient energy. Therefore, only electrons in the high-energy tail of the distribution function will be effective in excitation. However, low energy electrons do play an important role in further exciting electrons already in an excited energy level.

\subsection{1.b. The Role of Metastables}

Energy levels with long radiative lifetime are called metastable levels. Such states exist in neutral atoms, ions, and molecules. Metastable particles (atom and ion) are created by electron collisions and diffuse throughout the discharge while interacting with the neutral species as well as charged species. They can be destroyed by further collisions with electrons or other metastables, or by impact with the walls. For example in a low pressure helicon-wave excited plasma, the metastable argon ion density $\mathrm{Ar}^{+*}$ in the $3 \mathrm{p}^{4} 3 \mathrm{~d}$ manifold can become a significant fraction $(\sim 24 \%)$ of the ground state argon ion density. ${ }^{10}$ Furthermore, the cross sections for interactions with the metastable states are two to three orders of magnitude greater than those of the ground state. Thus, in low temperature plasmas it is important to include metastable states in any model of excited 


\section{Chapter 4: Plasma Diagnostics}

state populations due to the combination of their large cross sections and relatively low threshold energies.

\subsection{1.c. Electron Impact Ionization}

Perhaps the most important collisional process in plasma is ionization. Ionization occurs directly from the highly populated ground state as well as from excited states. Less collisional energy is required for ionization from an excited state as those levels are much closer to the continuum. Ionization for a neutral atom $A$ from an energy level $k$ (including the ground state) to the continuum can be represented by the following reaction:

$$
A_{k}+e^{-} \rightarrow A^{+}+2 e^{-}
$$

The ionization rate coefficients for this process can be calculated by integration of ionization cross section $\sigma_{i}(\varepsilon)$ over the electron energy distribution function. Because the electron distribution is a function of electron temperature, the ionization rate coefficient $k^{i z}$ is also a function of the electron temperature. When the ionization rate coefficient is known, the rate of direct ionization by electron impact is given by

$$
R^{i z}=k^{i z}\left(T_{e}\right) n_{e} n_{0},
$$

where $n_{e}$ is the electron density and $n_{0}$ is the neutral atom density. Since the ionization potential is usually much greater than the mean electron energy $\left(\langle\varepsilon\rangle=\int_{0}^{\infty} \varepsilon f(\varepsilon) d \varepsilon=3 T_{e} / 2\right)$, the ionization rate coefficient is very sensitive to the tail of the electron energy distribution function.

\subsection{1.d. Heavy Particle Impact Ionization}

An electron with slightly more kinetic energy than the ionization potential of a target species is quite effective at ionizing the target species. However, the same is not true for collisions of heavy particles, ions and neutrals, with a target species in the ground state. In a collision, a heavy particle is often unable to transfer sufficient energy for ionization to an electron inside an atom because the process is far from resonance. This 


\section{Chapter 4: Plasma Diagnostics}

situation changes significantly if incident heavy particle is in an excited state. When total electron excitation energy of the incident heavy particle is close to the ionization potential of the target species, the resonant energy transfer occurs and effective excitation or ionization ensues. Examples of collisional excitation processes include:

$$
A_{i}+A_{0}^{+} \rightarrow A_{j}+A_{0}^{+} \quad A_{i}^{+}+A_{0} \rightarrow A_{j}^{+}+A_{0},
$$

where $j>i$. Inelastic collisions that result in the ionization of the neutral atom are described by

$$
A_{i}+A_{0} \rightarrow A_{k}^{+}+A_{0}+e^{-} \quad A_{i}+A_{0}^{+} \rightarrow A_{k}^{+}+A_{0}^{+}+e^{-} .
$$

However, because the smaller rate coefficients and smaller population densities of the excited species with respect to the neutral and ion ground state populations, these processes can be neglected in a Corona model.

\subsubsection{Determination of Relative Ion Fractions from Optical Emission Spectroscopy (OES)}

Optical emission spectroscopy (OES) is perhaps the most widely used optical method for sensing atoms and small molecules in plasmas. With OES it is possible to determine the electron temperature, ion density, and spatial distributions of excited species. In plasma, gas-phase species are promoted to excited electronic states by collisions with energetic electrons and relaxation is accompanied by emission of a photon. In OES, the emitted radiation is spectrally dispersed and detected. In its simplest configuration, OES requires only a means of collecting the emitted light (e.g. an optical fiber), a dispersing element (a grating), and detector (a charge-coupled device - CCD). OES can be employed quantitatively and qualitatively for plasma species identification of absolute or relative species densities. Identification requires knowledge of the emission lines of a given plasma species. Although quantitative OES is possible, it must be used cautiously because signal intensity is not always directly related to concentration of the emitting states.

In low pressure, weakly ionized plasmas, the number of atoms (or ions) in an excited state can be calculated with the Corona Model described previously. In a Corona 
model, the species are excited solely by electron impact from ground or metastable states and are lost solely by radiative decay. In corona equilibrium, the electron excitation rate and decay rates are equal. For our low pressure plasma, quenching of excited state by the neutral gas can also be ignored for most excited species. Measurement of the relative intensities of the emission lines provides a qualitative indicator of species concentration. In calculating emission intensities of atomic and ionic lines with a Corona model, proper treatment of excitation from metastable states is critical. If the metastable density is known, the modeled intensity spectrum can include contributions from metastable and ground state excitation since most metastable excitation cross sections are known. Perhaps the biggest challenge in incorporating excitation and ionization from metastable states is that the electron energies required are much smaller than for excitation and ionization out of the ground state. Thus, the low energy electrons play an important role and the model must assume that the electron energy distribution function is characterized by the same electron temperature at high and low electron energies.

If the only excitation paths to an upper level $j$ are electron impact excitation from the ground and metastable states and the de-excitation path is radiative decay, the rate equation for the upper level $j$ is

$$
\frac{d n_{j}}{d t}=n_{e} n_{0} k_{0 \rightarrow j}+n_{e} n_{m} k_{m \rightarrow j}-\frac{n_{j}}{\tau_{j}},
$$

where $n_{e}, n_{0}, n_{m}$ and $n_{j}$ are the electron, neutral atom in the ground state, metastable state, and $j$ level densities; $\tau_{j}$ is the radiative lifetime of $j$ level; and $k_{0, m \rightarrow j}$ are the excitation rate coefficients for electron impact excitation from ground and metastable levels respectively to level $j$. The rate coefficients are given by Eq. (4.34 a) where the electron energy distribution function $f(\varepsilon)$ is obtained from Langmuir probe measurements. The cross sections to be used when computing optical emission intensities for a plasma are the optically measured values ${ }^{11}$, with no correction for optical cascade effects.

Assuming steady state in Eq. (4.36), the observed emission intensity $I_{j k}$ of species $A$ at wavelength $\lambda_{j k}$ corresponding to a quantum transition from upper level $j$ to a lower level $k$ is given by

$$
I_{j k}=\frac{h c}{4 \pi \lambda_{j k}} S\left(\lambda_{j k}\right) b_{j k} n_{e} n_{0} k_{0 \rightarrow j}\left(1+\frac{n_{m} k_{m \rightarrow j}}{n_{0} k_{0 \rightarrow j}}\right)
$$




\section{Chapter 4: Plasma Diagnostics}

where $S\left(\lambda_{j k}\right)$ is the detection system sensitivity (lenses, optical fibers, spectrometer, CCD camera) at $\lambda_{j k}, b_{j k}=A_{j k} / \sum_{l<j} A_{j l}$ is the branching ratio for the transition $j \rightarrow k, n_{0}$ is the number density in ground state, and $n_{m}$ is the number density in metastable state, $m$. If the relative intensities of two neutral atom lines of the same species are used, then the intensity ratio is a function only of the electron temperature. The situation for the relative intensity of ionic lines is much more complicated. Excitation to an excited ion state occurs by a one-step process (simultaneous excitation and ionization from the ground state of the neutral atom) or a two-step process (ionization of the neutral atom and then subsequent excitation). Since for a typical low temperature plasma, only few electrons have enough energy for the one-step process, the largest contribution to an excited ion state population is from excitation of ground state ions. Letting the upper level of the ion be denoted by $i$, and including both excitation pathways,

$$
\frac{d n_{i}^{+}}{d t}=n_{e} n_{0} k_{0 \rightarrow i}+n_{e} n_{0}^{+} k_{0 \rightarrow i}^{+}-\frac{n_{i}^{+}}{\tau_{i}},
$$

where $n_{0}{ }^{+}$is the number density of ion species in ground state and $k_{0 \rightarrow i}$ and $k_{0 \rightarrow i}^{+}$are the excitation rate coefficients for electron impact from neutral and ion ground state, respectively. For the particular case of an Ar-Xe plasma, the lowest lying ion metastable levels are at relatively low energies (13.48 eV for $\mathrm{Ar}^{+}$and $11.26 \mathrm{eV}$ for $\left.\mathrm{Xe}^{+}\right)$. Thus, the contribution to the excited ionic state population from metastable levels $n_{e} n_{m}^{+} k_{0 \rightarrow i}^{+}$has to be included in Eq. (4.38). One-step transitions to an excited ion state from the neutral ground state, the first term on the right-hand-side of Eq. (4.38), can be safely neglected ${ }^{12}$ for both $\mathrm{Ar}$ and $\mathrm{Xe}$ and the ion species emission line intensity is

$$
I_{i f}^{+}=\frac{h c}{4 \pi \lambda_{i f}} S\left(\lambda_{i f}\right) b_{i f} n_{e} n_{0}^{+} k_{0 \rightarrow i}^{+}\left(1+\frac{n_{m}^{+} k_{m \rightarrow i}^{+}}{n_{0}^{+} k_{0 \rightarrow i}^{+}}\right)
$$

With an electron energy distribution function obtained from Langmuir probe measurements, the excitation rate coefficients calculated from the excitation crosssections available in the literature, and OES measurements of line intensities, the relative ion densities of each species in a plasma mixture ( $\mathrm{Ar}$ and $\mathrm{Xe}$ for this study) can be estimated. 


\subsubsection{The OES Detection System}

The optical emission spectroscopy (OES) investigations reported in this thesis were performed with a $1.33 \mathrm{~m}$ Czerny-Turner double pass scanning monochromator (McPherson 209). The dispersive element is a $120 \times 140 \mathrm{~mm}$ grating having 1200 lines $/ \mathrm{mm}$ blazed at $750 \mathrm{~nm}$. The linear dispersion of this system is $0.62 \mathrm{~nm} / \mathrm{mm}$ and the maximum resolution is $0.15 \mathrm{~nm}$. Scanning over the range 400-900 nm is accomplished with a computer controlled stepper drive. The detector is an air cooled SBIG ST-7XEAI dual autofocusing CCD camera (Santa Barbara Instruments Group) with a $765 \times 510$ pixel array at $9 \mu \mathrm{m} /$ pixel that provides a spectral window of $3.5 \mathrm{~nm}$. The quantum efficiency of the camera is enhanced by the addition of a microlens array over the pixels. The quantum efficiency is 0.85 at $650 \mathrm{~nm}, \sim 0.45$ toward the blue $(400 \mathrm{~nm})$ and $\sim 0.05$ toward the near-infrared $(1000 \mathrm{~nm})$. The acquisition time of the camera ranges from $10^{-2}$ to several hundred seconds. Connection of the camera with a PC for data acquisition is through a high speed USB interface that allows transfer speeds of 1 frame/sec. A typical measurement of an Ar neutral line is shown in Figure 4.9.

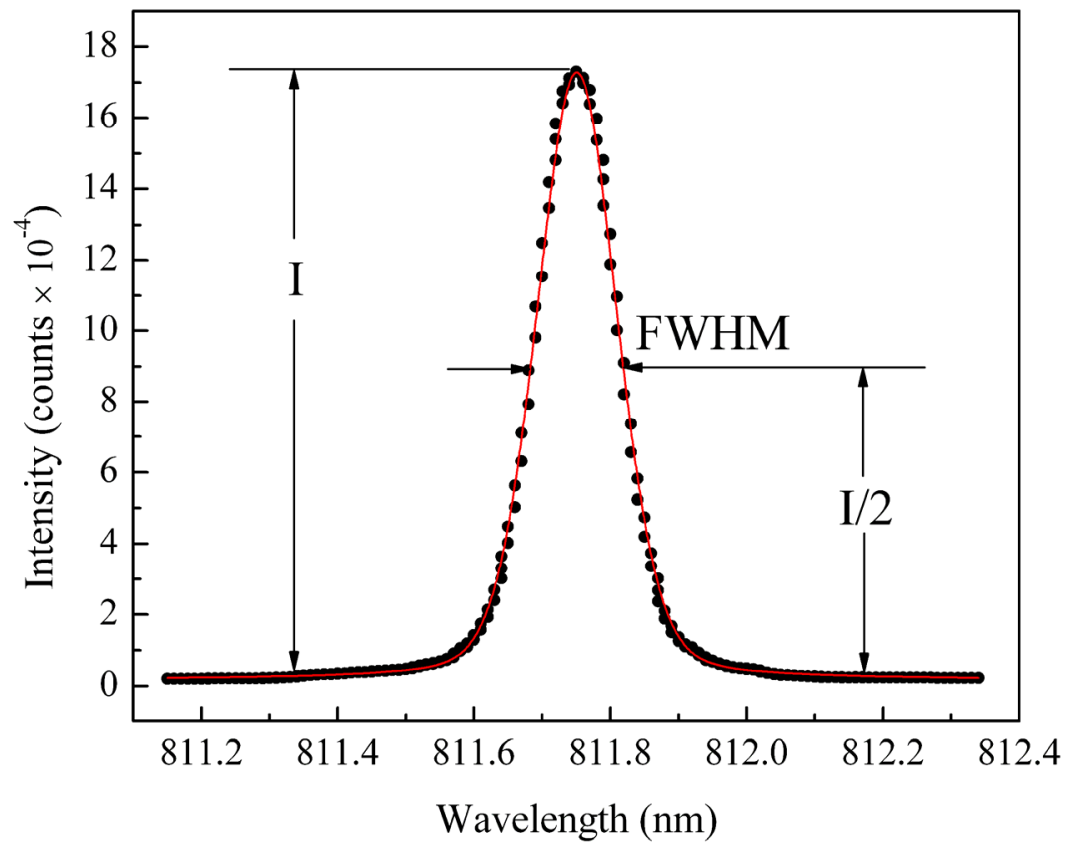

Figure 4.9. Typical Ar neutral line after background removal and spectral sensitivity correction. The fit to the experimental data is based on a pseudo Voight profile function. ${ }^{13}$ 
The light emitted by the helicon plasma source is focused by a series of $2.54 \mathrm{~cm}$ diameter lenses into a $200 \mu \mathrm{m}$ core multimode optical fiber and sent to the entrance slit of the spectrometer. The collection optics is aligned radially with the discharge and light is collected through radial ports at different axial locations along HELIX. For LEIA plasma investigations, collection optics mounted on a scanning probe (described later) was used. For all measurements, the width of the entrance slit was set to $80 \mu \mathrm{m}$ and the integration time was chosen so that none of the intensities were saturated. Wavelength calibration was performed with the help of an argon pen lamp and Ar neutral lines tables available from the NIST website. ${ }^{14}$
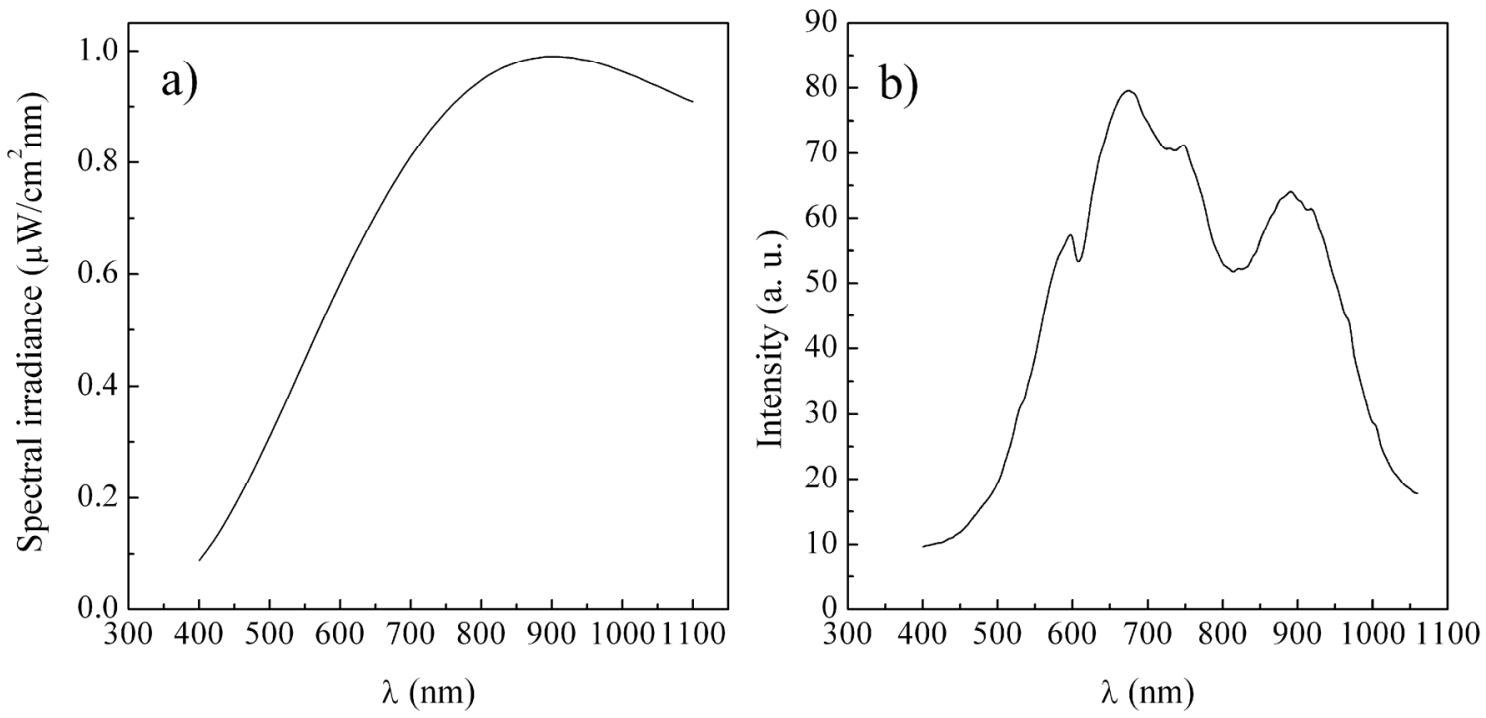

Figure 4.10. a) Spectral irradiance of the tungsten Oriel lamp; b) The measured response spectrum.

In this work, the integrated individual line intensities after background removal and spectral sensitivity correction were used (see Figure 4.9). Calibration of relative spectral response of the entire optical path was performed by using the tabulated spectral irradiance and the experimentally measured spectrum of a tungsten ribbon lamp $\left(\right.$ Oriel $\left.^{\mathrm{TM}}\right)$. Figure 4.10 shows the spectral irradiance of the tungsten ribbon lamp and the measured spectral intensity for the entire spectrometer achievable wavelength range of $400-1000 \mathrm{~nm}$. 


\subsection{Laser Induced Fluorescence (LIF)}

LIF is one of the most sensitive diagnostic methods available for the detection of atomic and ionic species. It is relatively easy to implement, phenomenological straightforward, well understood, and largely non-invasive; all characteristics that make LIF ideal for many applications. Some of the particular strengths of laser induced fluorescence are its high spatial and temporal resolution, and the ability to perform quantitative measurements. In practice, LIF is typically used to directly measure the ground (or metastable) state populations of active target species. To obtain the particle (atom, ion, or molecule) velocity distribution function (vdf) in steady state plasma, weak LIF emission is discriminated against background light either by external modulation of the laser beam or by using pulsed lasers and, in both cases, subsequent phase synchronous detection. The end results are precise particle velocity $(\sim 50 \mathrm{~m} / \mathrm{s})$ and temperature $(\sim 0.1 \mathrm{eV})$ measurements with high spatial resolution $\left(\right.$ few $\left.\mathrm{mm}^{3}\right)$.

\subsubsection{Continuous Wave LIF}

A velocity resolved, three-level LIF measurement consists of illumination of a distribution of particles (ions, atoms, radicals, molecules) with a laser whose bandwidth is much narrower than the particle's Doppler broadened absorption linewidth. If the incident photon has an appropriate frequency in the particle's rest frame, then a particular quantum level is optically pumped and the population of an upper quantum level increases. The upper level then decays spontaneously to a third level by emitting a photon. The intensity of the fluorescence radiation as a function of laser frequency is a direct measurement of the initial state particle velocity distribution (vdf), although processes such as Zeeman splitting may have to be included in the analysis of the data to determine the velocity distribution. In a typical LIF measurement, the particle ensemble velocity distribution function is obtained by scanning the laser frequency over the range of interest and recording the fluorescence spectrum. The measured vdf contains only onedimensional velocity information since the measurement is the projection of the $3 \mathrm{D} v \mathrm{vdf}$ on the laser propagation direction $\boldsymbol{k}_{\boldsymbol{L}}$. For example, when the laser is injected along the $x$ 
axis (see Figure 4.11), the measured $f\left(v_{x}\right)$ is an average over the distributions along the two directions orthogonal to $\boldsymbol{k}_{\boldsymbol{L}}$.

$$
f\left(v_{x}\right)=\iint f\left(v_{x}, v_{y}, v_{z}\right) d v_{y} d v_{z} .
$$

Determination of the average particle velocity and temperature from the LIF measurement is straightforward: $v_{x}$ is found from the peak in the measured $f\left(v_{x}\right)$ versus laser wavelength, and the temperature is obtained from the full width at half-maximum (FWHM) of the fluorescence line, assuming that Doppler broadening dominates over other line-broadening mechanisms

$$
k_{B} T_{x}=\left(m_{p} / 8 \ln 2\right)\left(\Delta v_{x}\right)_{1 / 2}^{2},
$$

where $m_{p}$ is the particle mass, $\left(\Delta v_{x}\right)_{1 / 2}$ the FWHM of the velocity distribution and $k_{B}$ is Boltzmann's constant.

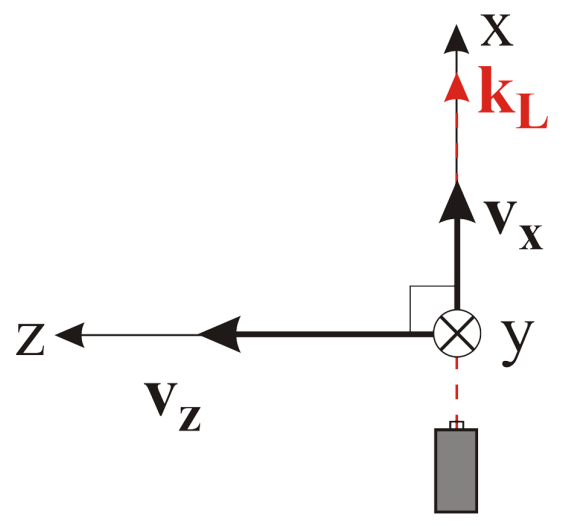

Figure 4.11. LIF injection parallel to $x$ axis for obtaining $f\left(v_{x}\right)$

High-velocity-resolution LIF of the ion velocity distribution function (ivdf) in plasma can be achieved a tunable, continuous wave (CW) ring dye laser. For $\mathrm{Ar}^{+}$, the measured LIF spectrum can be directly transformed into an excellent approximation of the ivdf. This is possible because the $\mathrm{Ar}^{+}$LIF spectrum is dominated by Doppler broadening. For our conditions, the Doppler broadening is much larger than the natural linewidth and Stark broadening. ${ }^{15}$ The effects of Zeeman broadening can be neglected in LEIA (low magnetic field) but do result in broadening and splitting of the line shape in HELIX (high magnetic field) for both $\mathrm{Ar}^{+}$and $\mathrm{Xe}^{+}$.

By suitable choice of laser polarization and laser injection relative to the magnetic field, it is possible to measure either parallel (to the magnetic field) or perpendicular ivdfs 


\section{Chapter 4: Plasma Diagnostics}

while minimizing the effects of Zeeman splitting of the absorption line. For perpendicular injection, the laser is polarized parallel to the field and only the $\pi$ Zeeman split lines are excited, corresponding to $\Delta M_{J}=0$ for $\mathrm{Ar}^{+}$and $\Delta M_{F}=0$ for $\mathrm{Xe}^{+}$). In the case of parallel injection, the laser light is converted into either right or left circularly polarized to excite the $\sigma^{+}$or $\sigma^{-}$Zeeman split lines, corresponding to $\Delta M_{J}= \pm 1$ for $\operatorname{Ar}^{+}$and $\Delta M_{F}= \pm 1$ for $\mathrm{Xe}^{+}$transitions.

\subsection{1.a. The LIF Diagnostic}

The LIF experimental setup shown in Figure 4.12 consists of a $100 \mathrm{MHz}$ linewidth, continuous wave Ar ion-pumped dye laser (Coherent 899 ring dye laser employing rhodamine $6 \mathrm{G}$ dye and pumped with a $6 \mathrm{~W}$ argon-ion laser) to excite transitions in both $\mathrm{Ar}^{+}\left(3 \mathrm{~d}^{2} \mathrm{G}_{9 / 2} \rightarrow 4 \mathrm{p}^{2} \mathrm{~F}^{0}{ }_{7 / 2}\right.$ at $\left.611.661 \mathrm{~nm}\right)$ and $\mathrm{Xe}^{+}\left(5 \mathrm{~d}^{4} \mathrm{D}_{7 / 2} \rightarrow 6 \mathrm{p}^{4} \mathrm{P}_{5 / 2}\right.$ at $605.278 \mathrm{~nm}$ ). The laser system includes a high resolution wavemeter Burleigh WA1500 for coarse tuning and wavelength monitoring. After passing through a beam splitter (BS), the laser beam is modulated with an optical chopper $(\mathrm{CH})$ at few $\mathrm{kHz}$ and then coupled into a multimode, non-polarization preserving, fiber optic cable. The $10 \%$ of the laser beam extracted before the optical chopper is passed through an iodine cell for a consistent zero velocity reference. Spontaneous emission from the iodine cell absorption lines is recorded with a photodiode (PHD) for each scan of the dye laser wavelength. The perpendicular injection optics includes a linear polarizer that allows selection of the laser polarization parallel to the magnetic field. The collection optics (CO) for parallel injection of laser light includes: a $2.54 \mathrm{~cm}$ collimating lens, followed a Galilean telescope for beam waist reduction, and a linear polarizer-quarter wave plate combination for conversion of the unpolarized laser light exiting the fiber into circularly polarized light to pump only $\sigma^{+}$transition cluster. The much smaller internal Zeeman splitting of the $\sigma$ lines is ignorable during analysis of the parallel LIF data for magnetic field strengths less than $1000 \mathrm{G}$. The fluorescence radiation from the plasma is collected at $90^{\circ}$ with respect to the laser beam and focused into a $200 \mu \mathrm{m}$-diameter fused silica optical fiber. The intersection of $5 \mathrm{~mm}$ diameter injected laser beam and the $0.8 \mathrm{~mm}$ diameter collection focal spot yields a measurement volume of $\approx 4 \mathrm{~mm}^{3}$. 


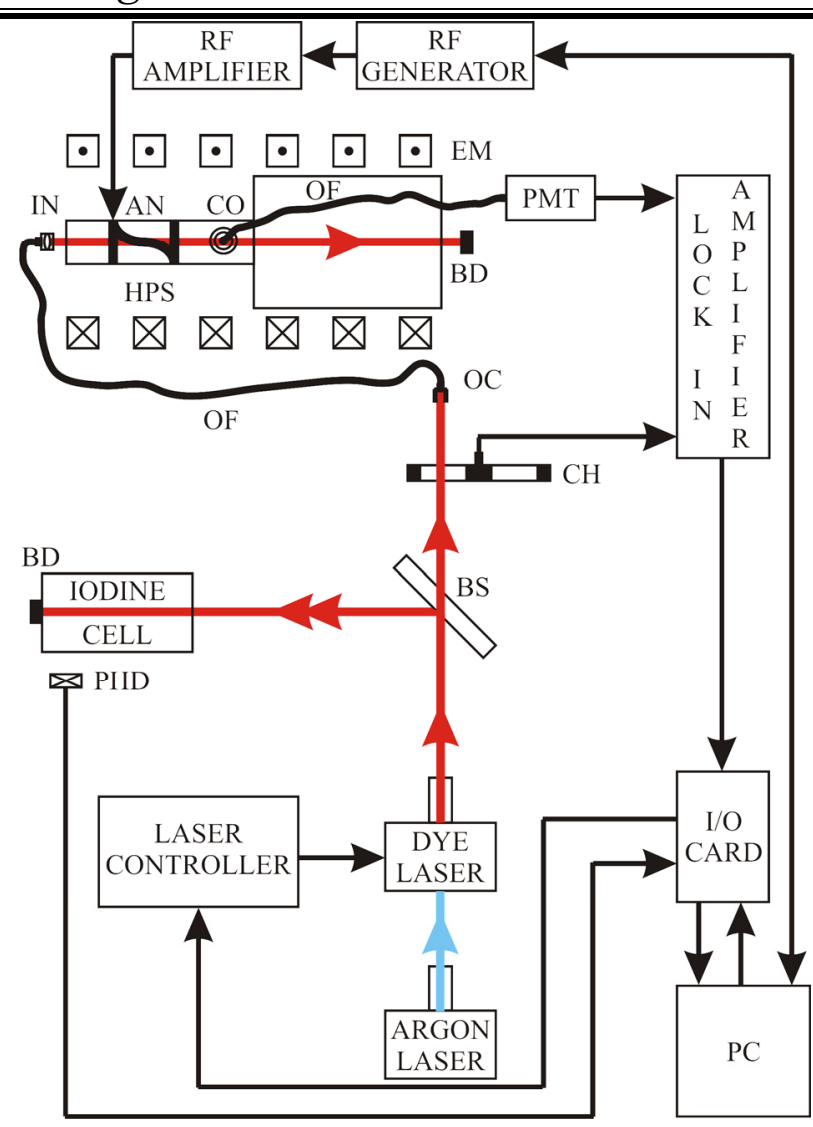

Figure 4.12. The LIF diagnostic: HPS -helicon plasma source, IN - injection optics, AN - antenna, CO collection optics, BD - beam dump, EM - electromagnets, PMT - photomultiplier tube, OF - optical fiber, $\mathrm{OC}$ - optical coupler, $\mathrm{CH}$ - chopper, $\mathrm{PHD}$ - photo diode, BS - beam splitter

Light exiting the collection fiber passes through 1-nm band pass interference filter centered at $461 \mathrm{~nm}$ for $\mathrm{Ar}^{+}\left(530 \mathrm{~nm}\right.$ for $\left.\mathrm{Xe}^{+}\right)$. Following the filter is a photomultiplier detector (PMT) with an integrated $30 \mathrm{kHz}$ bandwidth pre-amplifier. The PMT signal is composed of fluorescence radiation, electron impact induced radiation and electronic noise. A Stanford Research SR 830 lock-in amplifier, referenced to the modulation signal of the chopper, is used to isolate the LIF signal from background emission at the fluorescence wavelength.

\subsection{1.b. $\mathrm{Ar}^{+} \mathrm{LIF}$}

For $\mathrm{Ar}^{+}$LIF, the classic $\mathrm{Ar}^{+}$LIF scheme was used (see Figure 4.13). $\mathrm{The}^{+} \mathrm{Ar}^{+} 3 \mathrm{~d}$ ${ }^{2} \mathrm{G}_{9 / 2}$ metastable state ( $\tau=6.1 \mathrm{~s}$ lifetime ${ }^{16}$ ) is optically pumped by $611.661 \mathrm{~nm}$ (vacuum wavelength) laser light to the $4 \mathrm{p}^{2} \mathrm{~F}^{0}{ }_{7 / 2}$ state ( $\tau=8.5 \mathrm{~ns}$ lifetime ${ }^{17}$ ), which then decays to $4 \mathrm{~s}$ 
${ }^{2} \mathrm{D}_{5 / 2}$ state by emission at $461.086 \mathrm{~nm}$. Since the first state is metastable, the homogenous line width $\left(\Delta \omega_{0} / 2 \pi \approx \hbar / \tau\right)$ of the absorption process is dominated by the natural linewidth of the $4 \mathrm{p}^{2} \mathrm{~F}_{7 / 2}^{0}$ level. The resultant $1.9 \times 10^{-2} \mathrm{GHz}$ linewidth is infinitely small compared to typical Doppler broadened linewidths for $\mathrm{Ar}^{+}$; approximately $1 \mathrm{GHz}$ for argon ions at room temperature. The ion drift velocity in the laser propagation direction is determined from the shift of the LIF peak relative to the iodine signal after correcting for the Zeeman shift of the $\sigma^{+}$absorption line as the laser is swept over $20 \mathrm{GHz}$.

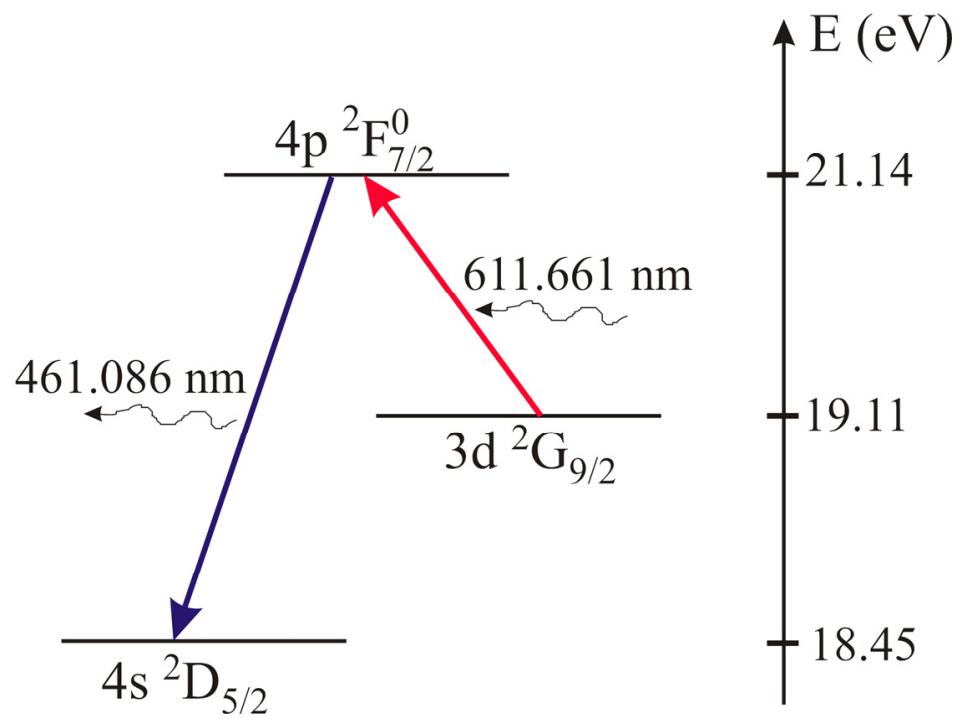

Figure 4.13. The three-level $\mathrm{Ar}^{+} \mathrm{LIF}$ scheme

For $\mathrm{Ar}^{+}$, the multiplet splitting caused by coupling of the electron-spin angular momentum $\mathbf{S}$ with the orbital angular momentum $\mathbf{L}$ (with a total electronic angular momentum $\mathbf{J}=\mathbf{L}+\mathbf{S}$ ) produces the fine structure shown in Figure 4.13. The interaction between the magnetic moment of the electronic states and an external magnetic field leads to Zeeman splitting of spectral lines. The Zeeman components and splitting of $\mathrm{Ar}^{+}$ absorption line at $611.661 \mathrm{~nm}$ are shown in Figure 4.14. The values of the splitting correspond to a magnetic field strength of $1 \mathrm{kG}$ in HELIX. ${ }^{18}$ The separation between the Zeeman components is proportional to the magnitude of the magnetic field and the Landé factor $\times$ magnetic orbital quantum number product: $\Delta \omega=(1 / \hbar) \mu_{B} B \Delta(g M)$, where $\mu_{B}=9.274$ $\mathrm{x} 10^{-24} \mathrm{~J} \mathrm{~T}^{-1}$. Zeeman splitting can yield a shift and, if unresolved, broadening of a spectral line. For our argon ion LIF pump line $\left(3 \mathrm{~d}^{2} \mathrm{G}_{9 / 2} \rightarrow 4 \mathrm{p}^{2} \mathrm{~F}^{0}{ }_{7 / 2}\right)$, Zeeman splitting of the $\Delta M_{J}= \pm 1$ transitions leads to $\sigma^{ \pm}$clusters (each of them consisting of eight lines) 
symmetrically shifted from the original wavelength. The $\Delta M_{J}=0$ transitions lead to an unshifted $\pi$ cluster of lines.

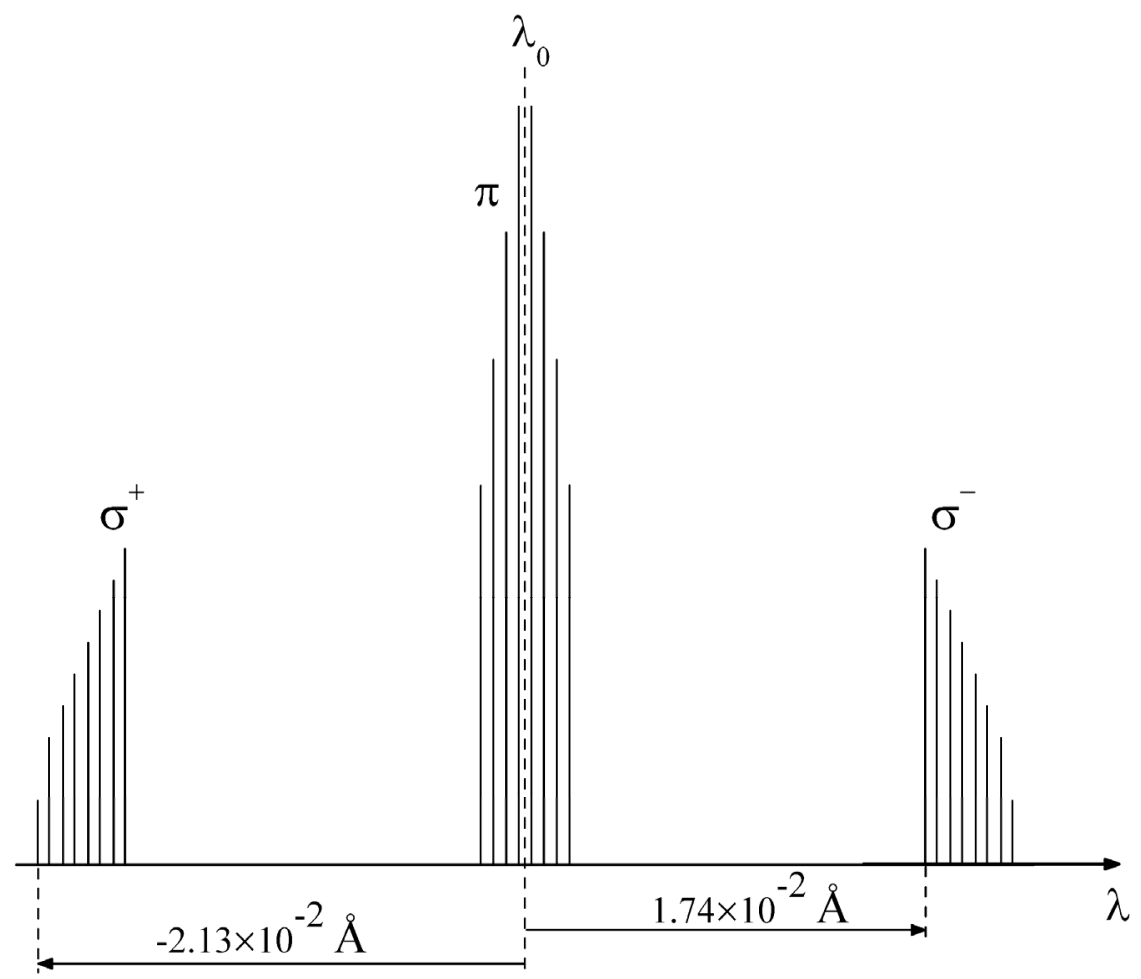

Figure 4.14. Schematic of the $\pi$ and $\sigma^{ \pm}$transitions for $\lambda_{0}=611.661 \mathrm{~nm} \mathrm{Ar}^{+}$line

Shown in Figures 4.15 (a) and (b) are two LIF measurements obtained in HELIX for a parallel laser injection (only the $\sigma^{ \pm}$clusters are pumped). Since the magnetic field strength is large enough in Figure 4.15 (b), the separation between the two clusters is clearly visible. The observed Zeeman splitting versus magnetic field strength shown in Figure 4.15 (c) has a slope of $1.52 \mathrm{GHz} / \mathrm{kGauss}$, very close to the predicted theoretical value of $1.48 \mathrm{GHz} / \mathrm{kGauss} .{ }^{19}$ Because the magnetic field strength at the measurement location (point $\mathrm{P}$ in Figure 3.4 a) is less than 180 Gauss, the Zeeman splitting of the $\sigma^{ \pm}$ $\left(\Delta M_{J}= \pm 1\right)$ and the $\pi\left(\Delta M_{J}=0\right)$ transitions result in a broadening of only $\approx 0.21 \mathrm{GHz}$, much smaller than the Doppler broadening and is therefore ignorable for calculations of ion temperature and flow velocity in LEIA. Stark broadening, power broadening, instrumental broadening, and the natural linewidth of the absorption line are also ignorable with respect to Doppler broadening, so the ion temperature is obtained from the FWHM of the distribution according to Eq. (4.41). Including the uncertainties introduced 
by ignoring Zeeman splitting, we estimate the experimental uncertainty in the ion temperature determination to be less than $0.05 \mathrm{eV}$.
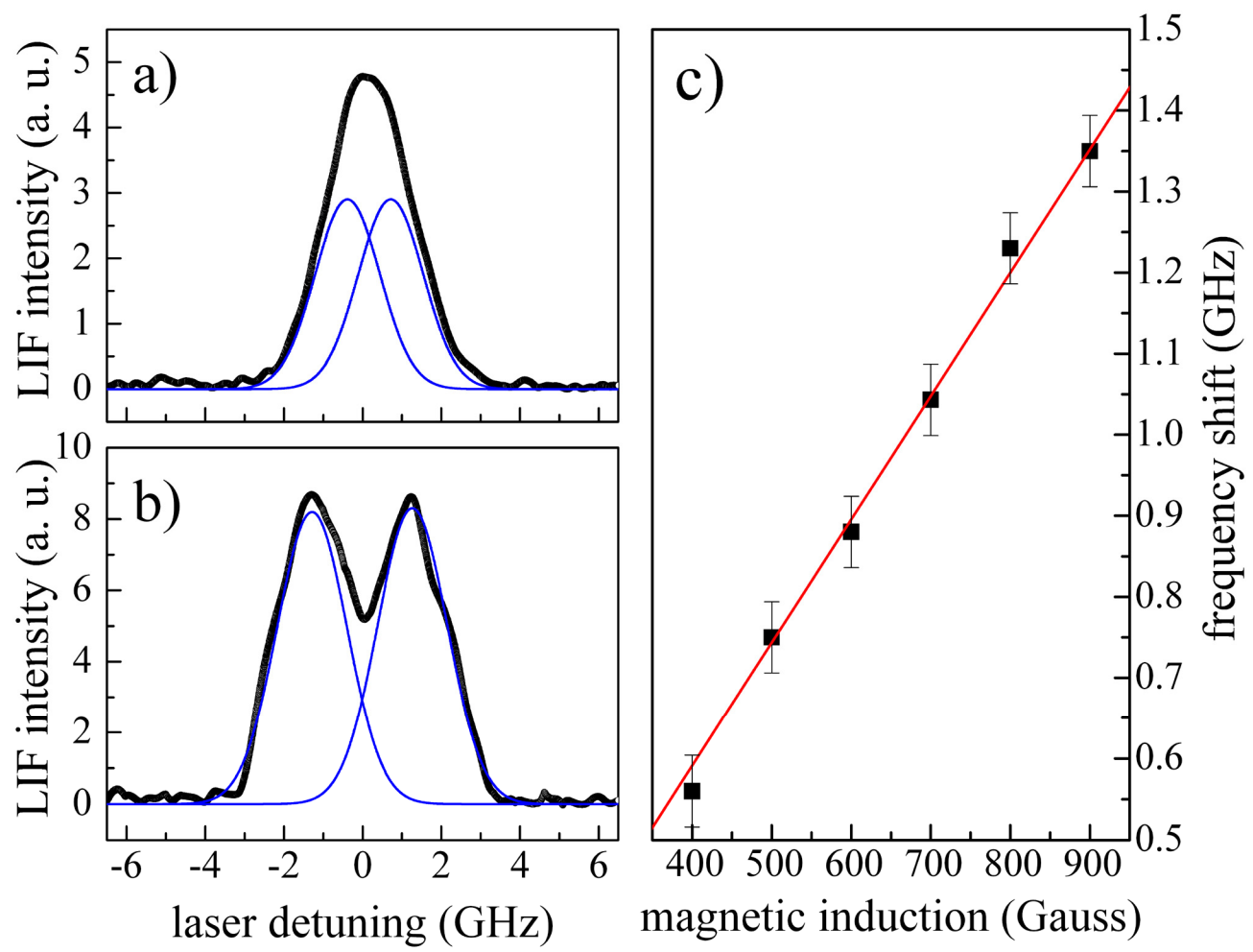

Figure 4.15. Evidence of Zeeman splitting in argon ion LIF measurements in HELIX for unpolarized laser injection along the magnetic field axis: a) LIF signal for an axial magnetic field of $0.4 \mathrm{kG}$; b) LIF signal for an axial magnetic field of $0.9 \mathrm{kG}$; $\mathrm{c}$ ) the dependence of $\sigma$ cluster splitting on the magnetic field strength.

\subsection{1.c. $\mathrm{Xe}^{+} \mathrm{LIF}$}

The same dye laser was used for $\mathrm{Xe}^{+}$LIF measurements. The $\mathrm{Xe}^{+}$LIF scheme is shown in Figure 4.16 (a). The laser wavelength was tuned to $605.278 \mathrm{~nm}$ to pump $\mathrm{Xe}^{+}$ from the metastable state $5 \mathrm{~d}{ }^{4} \mathrm{D}_{7 / 2}$ to the excited state $6 \mathrm{p}{ }^{4} \mathrm{P}_{5 / 2}$. Ions from $6 \mathrm{p}{ }^{4} \mathrm{P}_{5 / 2}$ state $\left(\tau=7.8 \mathrm{~ns}\right.$ lifetime $\left.{ }^{20}\right)$ decay to $6 \mathrm{~s}{ }^{4} \mathrm{P}_{5 / 2}$ state, emitting a $529.369 \mathrm{~nm}$ photon. The transition between the fine structure levels in $\mathrm{Xe}^{+}$is further split into a number of components lying extremely close together. Because this hyperfine splitting is significant, the absorption spectrum line shape in $\mathrm{Xe}^{+} \mathrm{LIF}$ is a convolution of the hyperfine splitting $H(v)$ and Doppler broadening function $D(v)$,

$$
I(v)=H(v) \otimes D(v) .
$$




$\begin{array}{lc}{ }^{129} \mathrm{Xe}^{+} & { }^{131} \mathrm{Xe}^{+} \\ \mathrm{I}=1 / 2 & \mathrm{I}=3 / 2\end{array}$
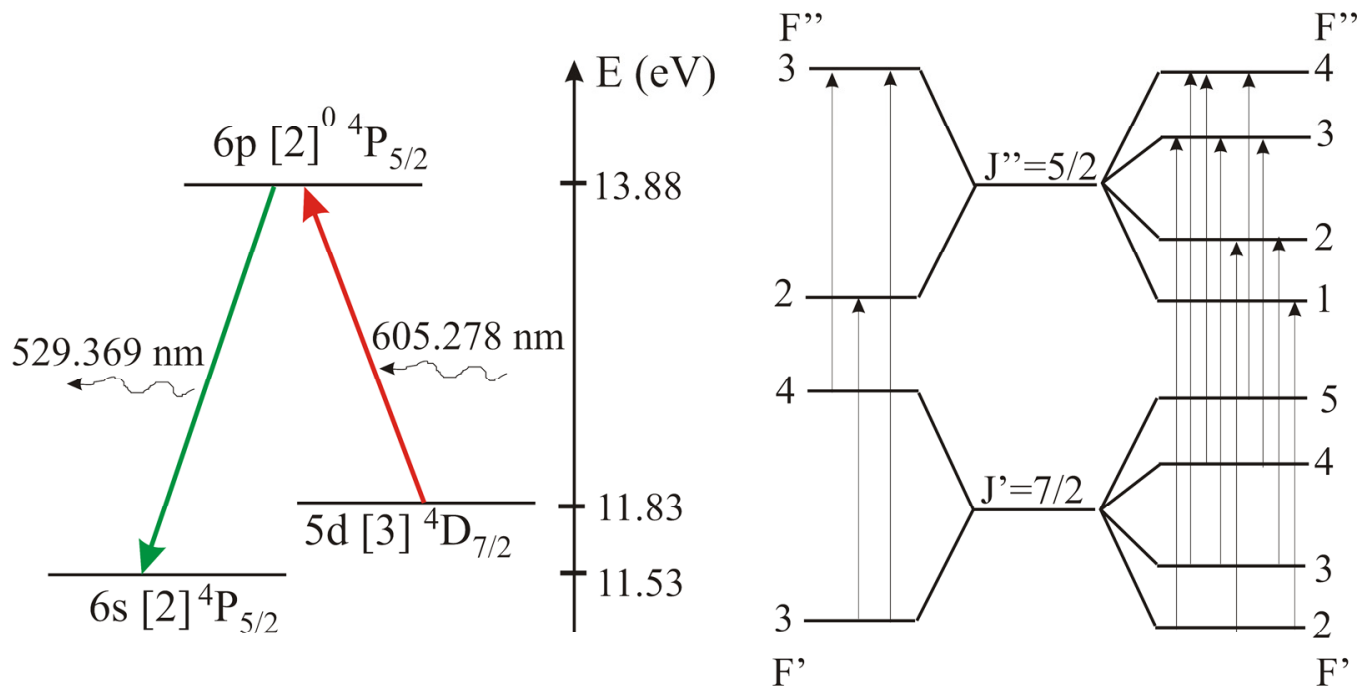

a)

b)

Figure 4.16. $\mathrm{Xe}^{+}$fine and hyperfine structure: a) $\mathrm{Xe}^{+}$LIF fine structure; b) Nuclear-spin structure of $605.278 \mathrm{~nm}$ line for two xenon isotopes.

The hyperfine structure of $\mathrm{Xe}^{+}$is a nuclear effect and includes the effects of mass (isotope effect) and nuclear spin (caused by the coupling between the nuclear spin $\mathbf{I}$ and the total electronic angular momentum $\mathbf{J}$ ).

\subsection{1.c.1. Isotopic Splitting of $\mathrm{Xe}^{+}$}

Xenon has a rich spectrum of isotopes, five of them having natural abundances of $10 \%$ or more. Each of these isotopes causes a shift of the energy levels, isotopic splitting, involved in a transition of a few tens of MHz. Figure 4.17 depicts the naturallyoccurring xenon isotopic abundances and shifts for the $5 \mathrm{~d}{ }^{4} \mathrm{D}_{7 / 2} \rightarrow 6 \mathrm{p}{ }^{4} \mathrm{P}_{5 / 2}$ transition relative to same transition in ${ }^{132} \mathrm{Xe}^{+}$. 


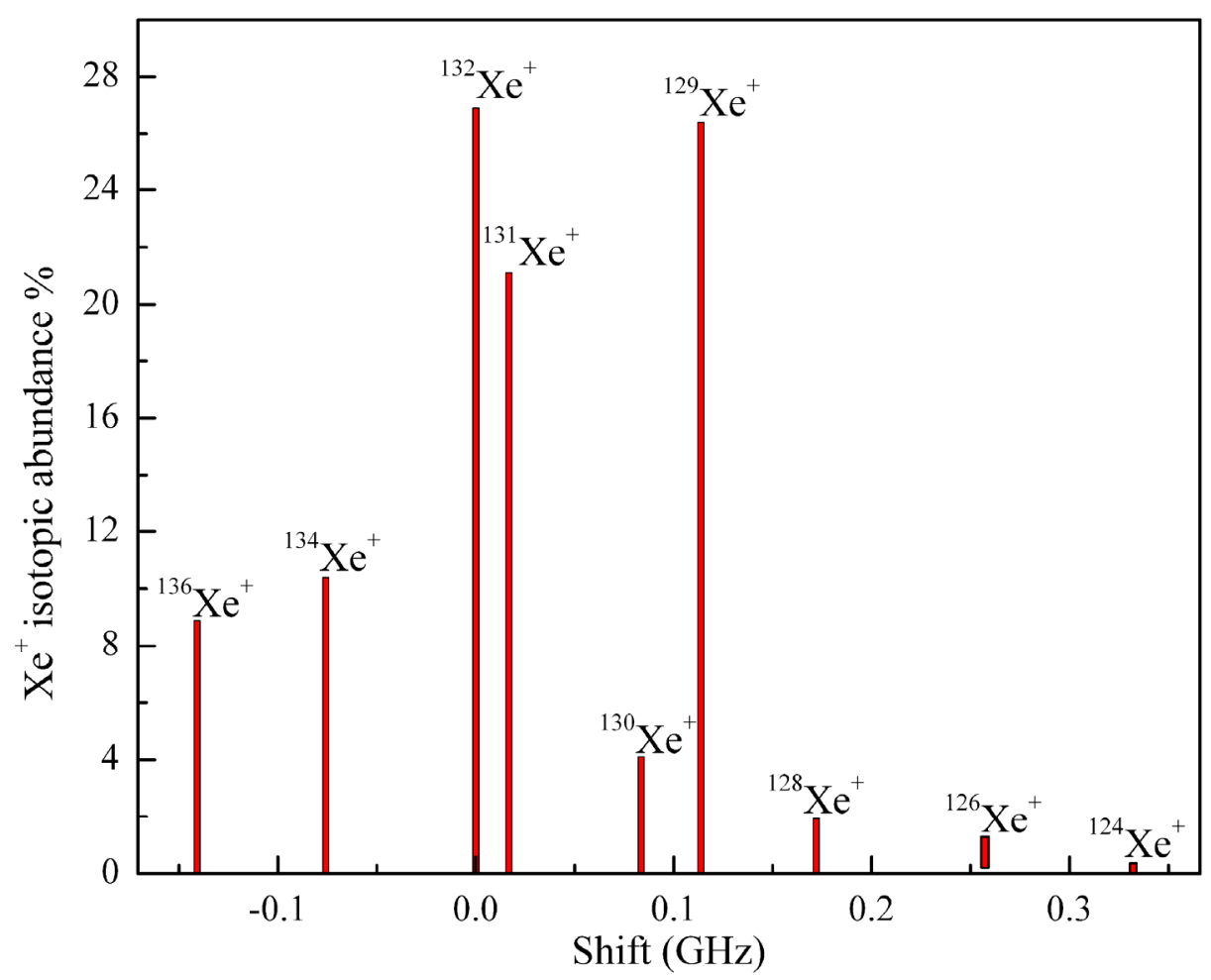

Figure 4.17. $\mathrm{Xe}^{+}$isotopic abundance ${ }^{21}$ and shifts relative to ${ }^{132} \mathrm{Xe}^{+}$of the $5 \mathrm{~d}^{4} \mathrm{D}_{7 / 2} \rightarrow 6 \mathrm{p}^{4} \mathrm{P}_{5 / 2}$ transition. ${ }^{22,23}$

\subsection{1.c.2. Nuclear-Spin Splitting of $\mathrm{Xe}^{+}$}

Of the nine isotopes of xenon, seven have an even atomic mass. Thus, they have a nuclear spin of $\mathbf{I}=0$ and do not contribute to the nuclear-spin splitting. The remaining two isotopes have odd atomic masses and non-zero nuclear spin quantum numbers $\mathbf{I}$. While the lighter isotope, ${ }^{129} \mathrm{Xe}^{+}$, has $\mathrm{I}=1 / 2,{ }^{131} \mathrm{Xe}^{+}$has $\mathrm{I}=3 / 2$. These non-zero nuclear spins cause nuclear spin splitting of the energy levels. This nuclear-spin splitting is considerably larger than the isotopic splitting and is responsible for most of the characteristic shape of the $5 \mathrm{~d}^{4} \mathrm{D}_{7 / 2} \rightarrow 6 \mathrm{p}{ }^{4} \mathrm{P}_{5 / 2}$ line.

For $\mathrm{Ar}^{+}$, L-S coupling was used to determine the total angular momentum $\mathbf{J}$. For $\mathrm{Xe}^{+}, \mathbf{J}$ and $\mathbf{I}$ must be combined to give the total angular momentum $\mathbf{F}=\mathbf{J}+\mathbf{I}$. The corresponding total angular quantum number $F$ and the component $M_{F}$ of the total angular momentum have values of ${ }^{24}$

$$
\begin{gathered}
F=J+\mathrm{I}, J+\mathrm{I}-1, \ldots \ldots,|J-\mathrm{I}|, \\
M_{F}=F, F-1, \ldots \ldots,-F
\end{gathered}
$$


For ${ }^{129} \mathrm{Xe}^{+}$, each level splits up into a hyperfine doublet (see Figure $4.16 \mathrm{~b}$ ). For ${ }^{131} \mathrm{Xe}^{+}$, the levels split up into four hyperfine levels (see Figure $4.16 \mathrm{~b}$ ). The energy due to nuclear-spin splitting is given by ${ }^{25}$

$$
E_{n s s}=\frac{A}{2} X+B Y,
$$

where $A$ is the nuclear magnetic dipole interaction constant, $B$ is the nuclear electric quadrupole constant, and the terms $X$ and $Y$ include the nuclear-spin orbit interaction.

$$
\begin{aligned}
& X=F(F+1)-I(I+1)-J(J+1) \\
& Y=\frac{(3 X / 4)(X+1)-I(I+1) J(J+1)}{2 I(2 I-1) J(2 J-1)} .
\end{aligned}
$$

This model uses Broström's nuclear-spin structure constants for $\mathrm{Xe}^{+} 5 \mathrm{~d}^{4} \mathrm{D}_{7 / 2}$ and $6 \mathrm{p}{ }^{4} \mathrm{P}_{5 / 2}$ energy levels. ${ }^{26}$ The selection rules for nuclear-spin splitting are $\Delta F=[0, \pm 1]$, where $\Delta F$ is defined as the difference between the lower $\left(F^{\prime}\right)$ and upper $\left(F^{\prime \prime}\right)$ state total angular quantum number. The transition $F^{\prime}=0 \rightarrow F^{\prime \prime}=0$ is forbidden. Using the above selection rules, the number of nuclear-spin split transitions can be determined. Figure $4.16 \mathrm{~b}$ ) shows that the $\mathrm{Xe}$ transition $5 \mathrm{~d}^{4} \mathrm{D}_{7 / 2} \rightarrow 6 \mathrm{p}{ }^{4} \mathrm{P}_{5 / 2}$ at $605.278 \mathrm{~nm}$ has three components for ${ }^{129} \mathrm{Xe}^{+}$and nine components for ${ }^{131} \mathrm{Xe}^{+}$. The relative intensities of each nuclear-spin split component for $J \rightarrow J-1$ transition are given by ${ }^{27}$

$$
\begin{aligned}
& i(F \rightarrow F-1) \propto \frac{[(F+J)(F+J+1)-I(I+1)][(F+J)(F+J-1)-I(I+1)]}{F} \\
& i(F \rightarrow F) \propto-\frac{(2 F+1)[(F+J)(F+J+1)-I(I+1)][(F-J)(F-J+1)-I(I+1)]}{F(F+1)} \\
& i(F-1 \rightarrow F) \propto \frac{[(F-J)(F-J+1)-I(I+1)][(F-J)(F-J-1)-I(I+1)]}{F}
\end{aligned}
$$

Resolving all the isotope splitting and determining the assignments for the nuclear-spin split lines from a single measured spectrum is impractical. For this reason, the hyperfine splitting $H(v)$ in Eq. (4.42) is modeled as a sum of nineteen individual hyperfine splitting functions $h_{i}(v)$, each of them with a magnitude proportional to the relative line intensity of each spin-split component $i_{i}$ [25].

$$
h_{i}(v)=i_{i} \delta\left(v_{i}-v_{0}\right) \text {, }
$$


where $v_{i}$ is the center of $i$-th component hyperfine line, $v_{0}$ is the line frequency for ${ }^{132} \mathrm{Xe}^{+}$, and $\delta$ is Dirac delta function.

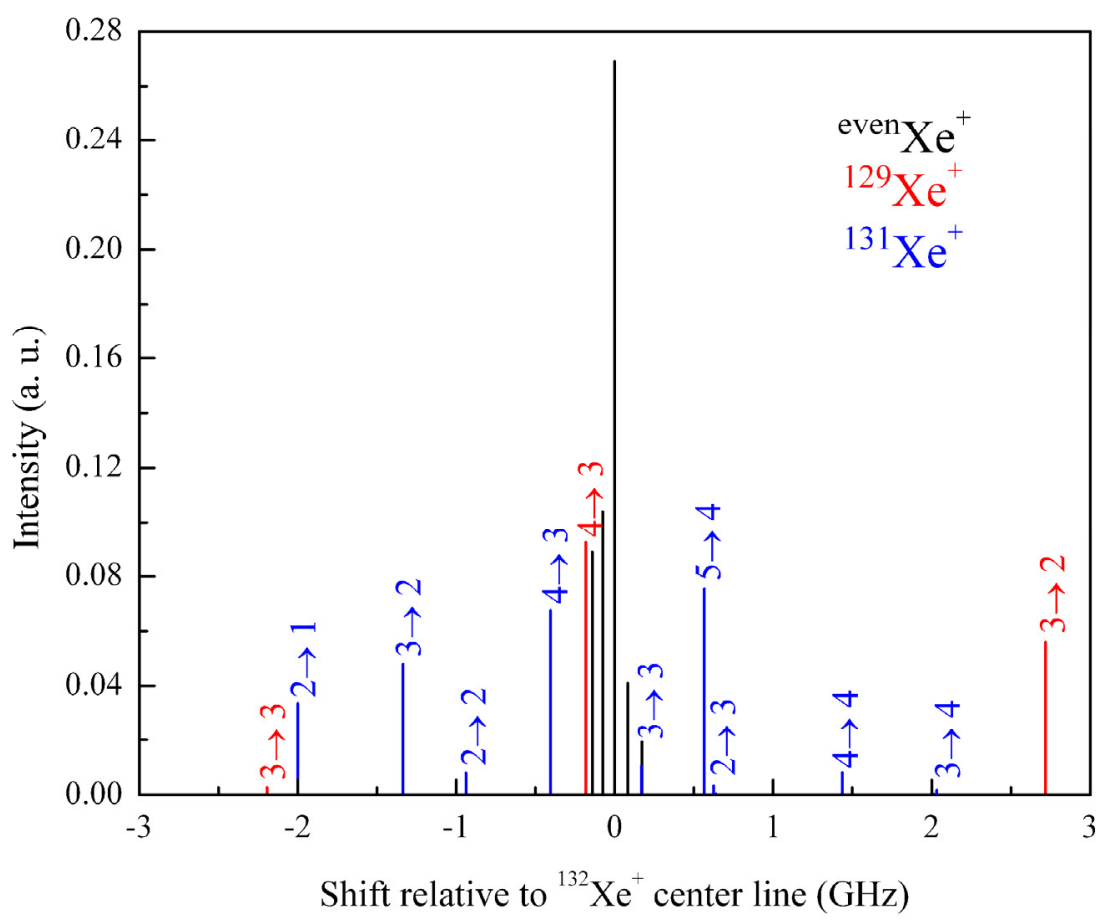

Figure 4.18. $\mathrm{Xe}^{+} 5 \mathrm{~d}^{4} \mathrm{D}_{7 / 2} \rightarrow 6 \mathrm{p}{ }^{4} \mathrm{P}_{5 / 2}$ hyperfine line splitting. The transition label on vertical line is $\mathrm{F}^{\prime} \rightarrow$ F".

The relative intensities of the isotopes without nuclear-spin splitting are given by their isotopic abundance. ${ }^{28}$ The line intensities of ${ }^{129} \mathrm{Xe}^{+}$and ${ }^{131} \mathrm{Xe}^{+}$assumed to be proportional to the product of their isotopic abundances and the relative intensities of the nuclear-spin split components evaluated from Eq. (4.46 - 4.48). The resultant hyperfine splitting of $\mathrm{Xe}^{+} 5 \mathrm{~d}^{4} \mathrm{D}_{7 / 2} \rightarrow 6 \mathrm{p}{ }^{4} \mathrm{P}_{5 / 2}$ line is shown in Figure 4.18.

In the case of weak to moderate external magnetic field strengths, the hyperfine structure is further split in $2 F+1$ sublevels. By analogy with the fine structure, Zeeman splitting for the $\mathrm{F}^{\prime}=3 \rightarrow \mathrm{F}^{\prime \prime}=2$ transition for ${ }^{129} \mathrm{Xe}^{+}$(see Figure 4.19) and ${ }^{131} \mathrm{Xe}^{+}$yields four $\pi$-lines for which $\Delta M_{F}=0$ and ten $\sigma$-lines for which $\Delta M_{F}= \pm 1$. 


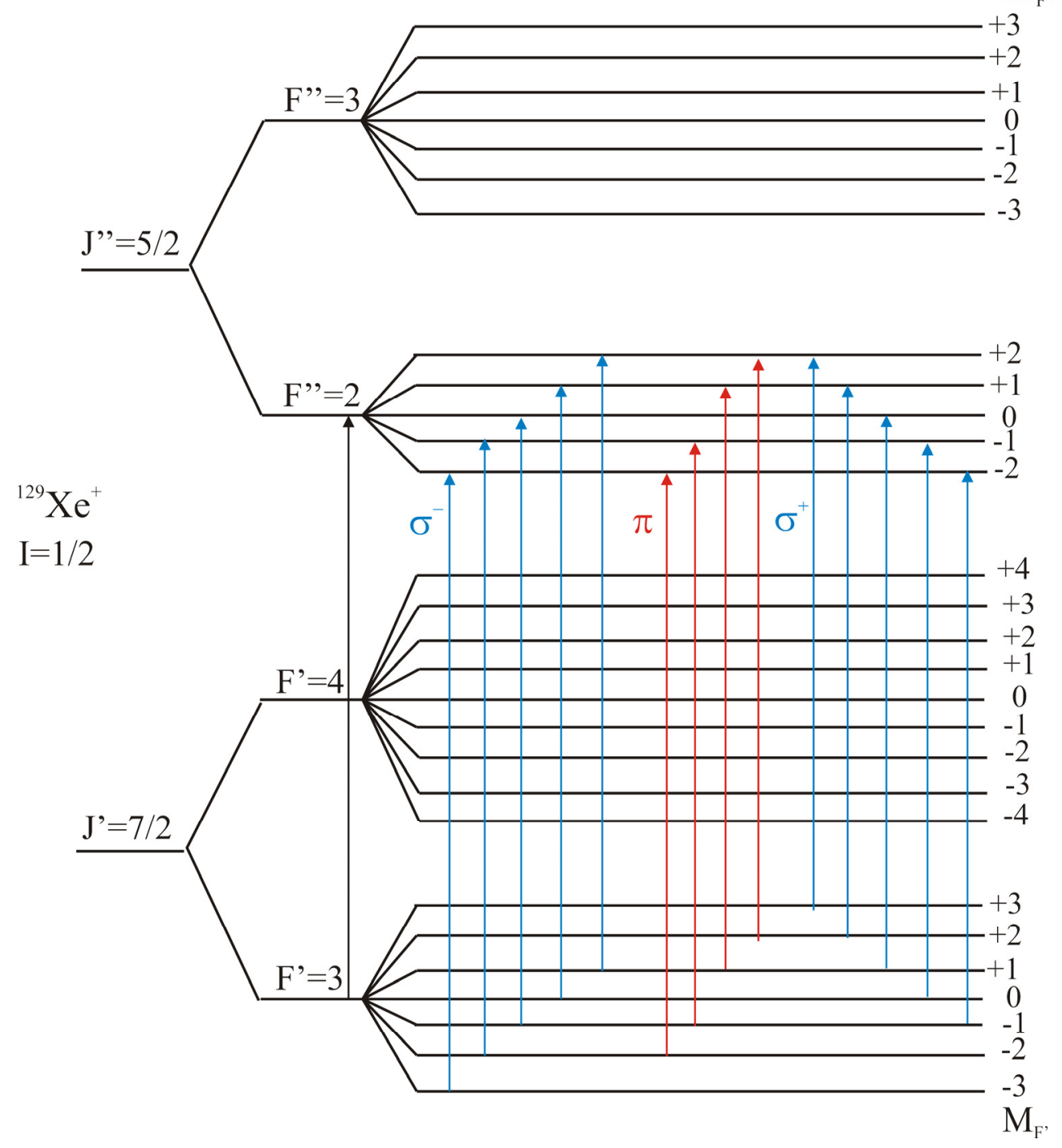

Figure 4.19. ${ }^{129} \mathrm{Xe}^{+} 5 \mathrm{~d}^{4} \mathrm{D}_{7 / 2}\left(\mathrm{~F}^{\prime}=3\right) \rightarrow 6 \mathrm{p}^{4} \mathrm{P}_{5 / 2}\left(\mathrm{~F}^{\prime \prime}=2\right)$ Zeeman line splitting

The first order energy contribution is given by:

$$
E_{Z}=M_{F}\left(g_{F} \mu_{B}-g_{N} \mu_{N}\right) B
$$

where $\mu_{B}$ and $\mu_{N}$ are the Bohr magneton and the nuclear magneton, respectively. The Landé $g$-factors are given by:

$$
\begin{aligned}
& g_{F}=g_{J} \frac{F(F+1)+J(J+1)-I(I+1)}{2 F(F+1)} \\
& g_{J}=1+\frac{J(J+1)-L(L+1)+S(S+1)}{2 J(J+1)}
\end{aligned}
$$




$$
g_{N}=g_{I} \frac{F(F+1)-J(J+1)+I(I+1)}{2 F(F+1)}
$$

The second term in Eq. (4.49) can be neglected since $g_{I} \mu_{N}$ is $m_{p} / m_{e} \approx 1800$ times smaller than $g_{J} \mu_{B}$. Eq. (4.49) indicates that every hyperfine level will be split into a number of equidistant sublevels, each of which will have an energy splitting proportional to the magnetic field strength:

$$
\Delta E_{Z}=\left(M_{F^{\prime}} g_{F^{\prime}}-M_{F^{\prime \prime}} g_{F^{\prime \prime}}\right) \mu_{B} B
$$

Based on Eq. (4.50), the calculated Zeeman shifts for a magnetic field strength of $1 \mathrm{kG}$ are shown in Figure 4.20. The hyperfine transition used was $F^{\prime}=3 \rightarrow F^{\prime \prime}=2$ for the ${ }^{129} \mathrm{Xe}^{+}$and ${ }^{131} \mathrm{Xe}^{+}$isotopes.

Complete analysis of a $\mathrm{Xe}^{+} \mathrm{LIF}$ measurement requires accounting for Doppler broadening as well as the hyperfine, isotopic, and possibly Zeeman structures of the $5 \mathrm{~d}$ ${ }^{4} \mathrm{D}_{7 / 2} \rightarrow 6 \mathrm{p}^{4} \mathrm{P}_{5 / 2}$. 


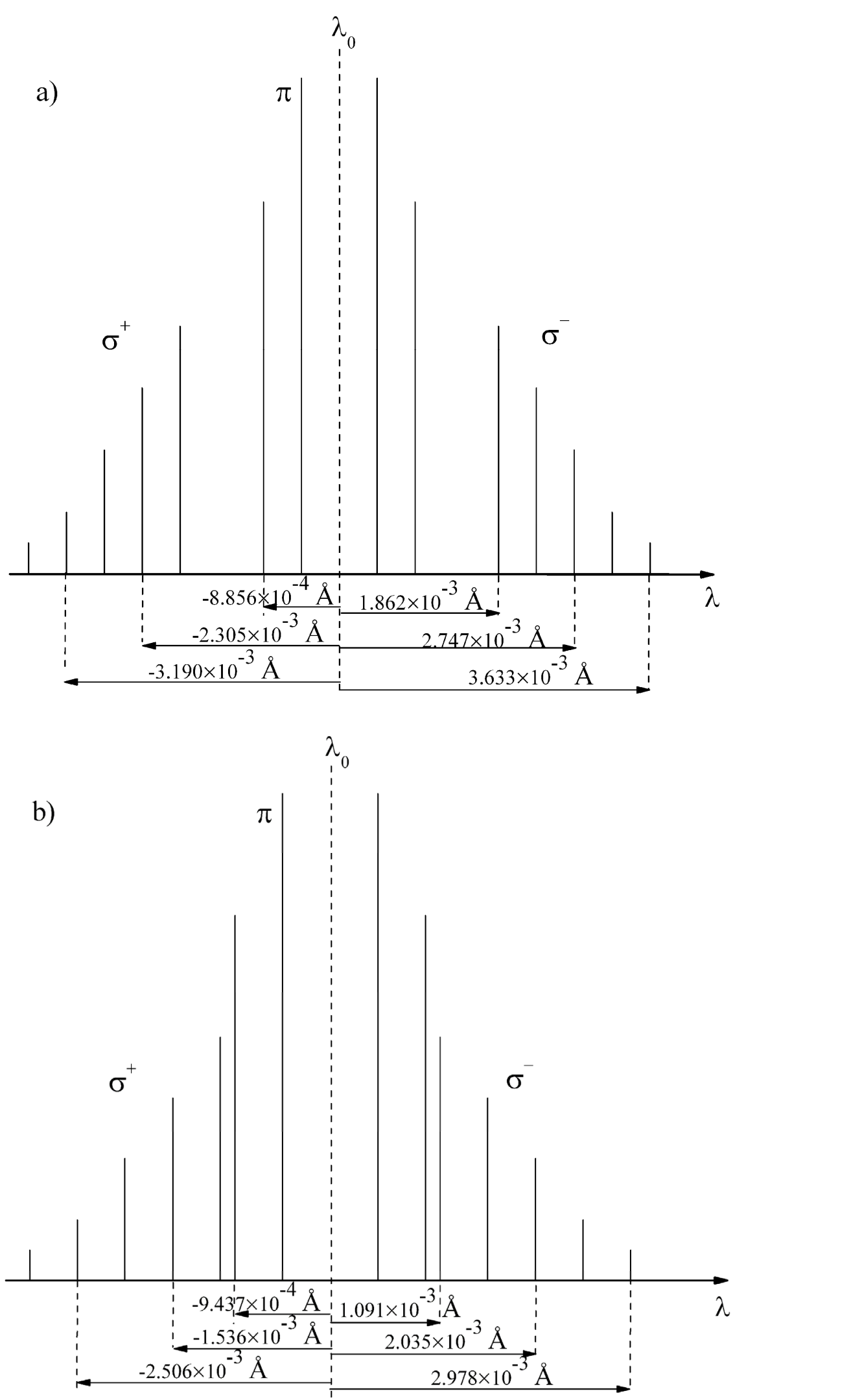

Figure 4.20. ${ }^{129} \mathrm{Xe}^{+}$(a) and ${ }^{131} \mathrm{Xe}^{+}$(b) Zeeman shifts for $\mathrm{F}^{\prime}=3 \rightarrow \mathrm{F}$ ' $=2$ hyperfine transition. $\lambda_{0}=605.278 \mathrm{~nm}$ 


\subsubsection{Time Resolved LIF}

To investigate transient phenomena with short characteristic time scales, high time resolution LIF methods must be employed. Since the lower temporal bound of an LIF measurement is set by the lifetime of the upper optically pumped level (usually few nanoseconds), time resolutions as high as few ns could theoretically be achieved. In practice, however, the time resolution is limited by needing to collect a sufficient number of LIF photons for a reasonable signal to noise; the RC time constants of electrical components of the LIF system; signal acquisition speed of the available electronics; and the particular plasma conditions. Time resolved LIF measurements with $0.2-20 \mu \mathrm{s}$ resolution have been performed by employing a two-channel box car integrator/averager ${ }^{29}$ or a multichannel scaler as a discriminator. ${ }^{30,31}$ In previous work we demonstrated LIF time resolutions of $1 \mathrm{~ms}$ using a standard lock-in amplifier and a fast digital oscilloscope, for both a ring dye laser and a low power tunable diode laser. ${ }^{32,33}$ The $1 \mathrm{~ms}$ resolution limit arose from the requirement that the mechanical chopping frequency be a few times faster than the lock-in integration time (for reasonable signalto-noise levels). An improvement in the LIF time resolution of a factor of $\sim 30$ was made possible by replacing the mechanical chopper with a high-speed acousto-optic modulator and by digital signal processing of the raw data. The experiments were performed in a pulsed plasma source. The $5 \mathrm{~Hz}$ pulsed operation was accomplished by amplitude modulation of the $9.5 \mathrm{MHz}$ driving frequency. A schematic of the argon ion LIF system used for parallel ivdf measurements in pulsed helicon plasma is shown in Figure 4.21. The laser beam is modulated with an acousto-optic modulator (AOM) at $100 \mathrm{kHz}$ and then coupled into a multimode, non-polarization preserving, fiber optic cable as described previously. A high-frequency lock-in amplifier provided the reference modulation signal to the AOM driver. The high-speed real and imaginary portions of the lock-in amplifier output were recorded with a digital oscilloscope synchronized to the rf modulation of the plasma source. The lock-in signals were averaged over few hundred plasma pulses and sampled at the digitization rate of the oscilloscope. 


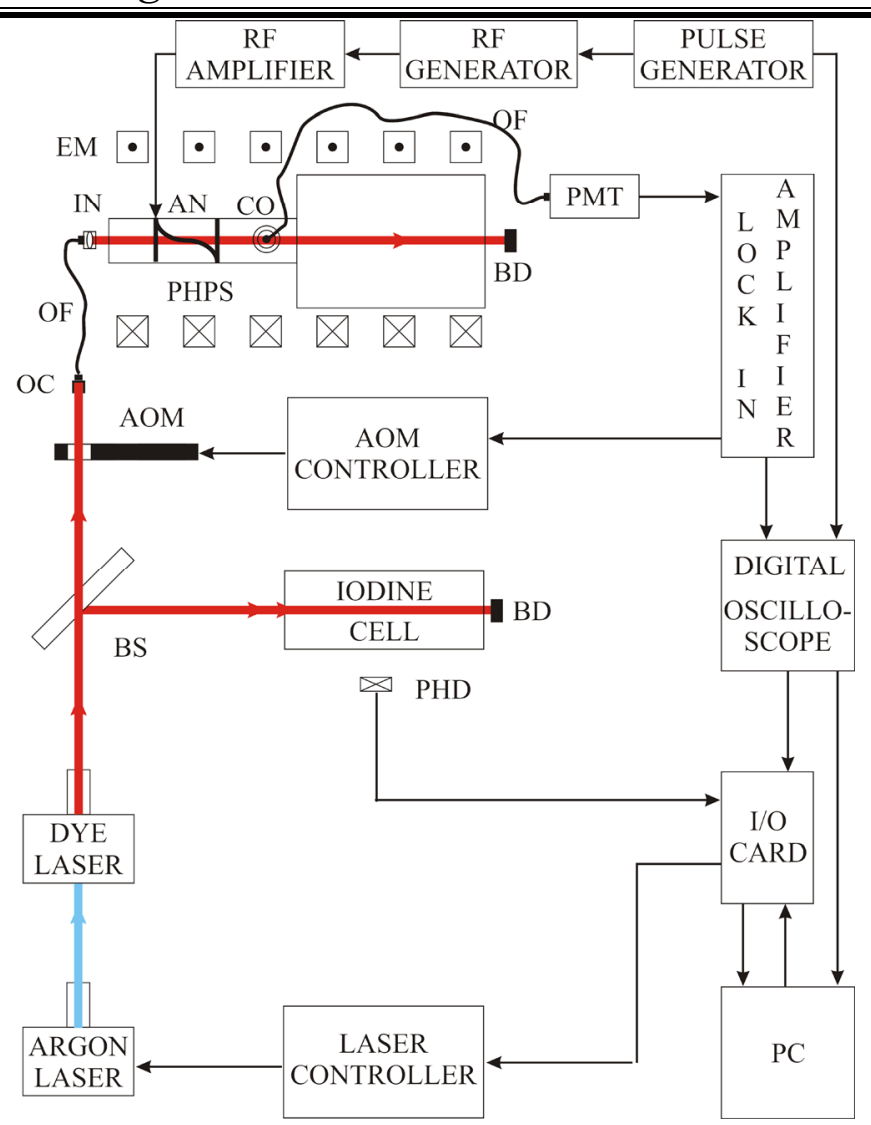

Figure 4.21. Experimental set-up for time resolved $\mathrm{Ar}^{+}$LIF diagnostic: PHPS - pulsed helicon plasma source, AOM - acousto-optic modulator

The time resolution of the averaged signal is limited by the integration time setting of the lock-in amplifier and the digitization rate of the oscilloscope. For a reasonable signal to noise ratio, the minimum necessary "on/off" cycles within the lock-in integration time for a mechanical chopper (4 kHz chopping frequency) was 3-4. Since the AOM was driven directly by the lock-in amplifier at up to $100 \mathrm{KHz}$, requiring three "on/off" cycles limited the integration time no less than $30 \mu \mathrm{sec}$. However, the LIF signals were recorded at a digitization rate of $10 \mathrm{kHz}$ to obtain a data record long enough to cover the entire plasma pulse, thus limiting the time resolution to $100 \mu \mathrm{sec}$. A significant challenge presented by this technique is that the transmitted laser power is significantly lower with the AOM than with the mechanical chopper. The transmitted intensity in the first diffraction order is a sensitive function (see Figure 4.22 a) of Bragg angle alignment ${ }^{34}$

$$
I_{1} / I_{0} \propto\left[\sin ^{2}\left(\pi \delta / \theta_{B}\right)\right] /\left(\pi \delta / \theta_{B}\right)^{2},
$$


where $I_{l}$ and $I_{0}$ are the intensity of the first order beam and the intensity of the zeroth order beam when the acoustic energy in the AOM medium is zero, respectively, $\theta_{\mathrm{B}}=\lambda f / 2 \mathrm{v}$ ( $\lambda$ is the laser wavelength in vacuum, $f$ is the acoustic frequency, $v$ is acoustic velocity in the AOM medium) is the Bragg angle, and $\delta$ the angular misalignment with respect to $\theta_{\mathrm{B}}$. For our AOM (Isomet 1205C-2 crystal with a Isomet 222A1 driver), $I_{1}$ is $\sim 35 \%$ of $I_{0}{ }^{35}$. Since during the AOM "on" interval, only $\sim 10 \%$ of the light remains in the $m=0$ order, we chose to inject light from the $m=0$ beam that was modulated between $100 \%$ (during the "off" interval) and $10 \%$ (during the "on" interval). Losses in the injected light path, particularly in coupling into the fiber, reduce the injected light to $20-30 \%$ of the laser output. Saturation can complicate the line shape analysis of the transitions since it occurs when the laser light is intense enough to pump most of the ions out of the initial LIF state, thus the remaining ions in the initial level are unable to absorb all the incident laser light and LIF signal saturates. However, for these levels of injected power $(\sim 40 \mathrm{~mW})$, the LIF was in a linear regime (see Figure 4.22 b), i.e. the measurements showed no sign of saturation.

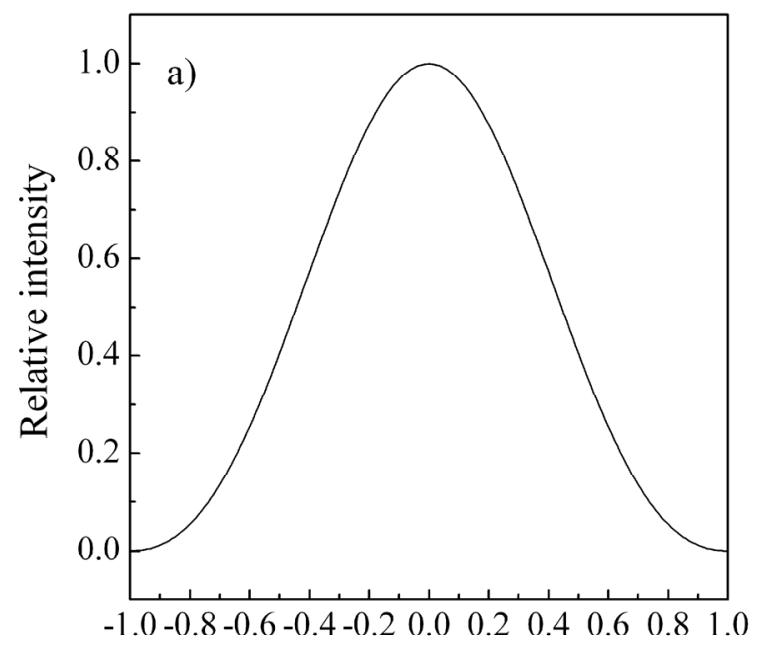

Normalized Bragg angle error

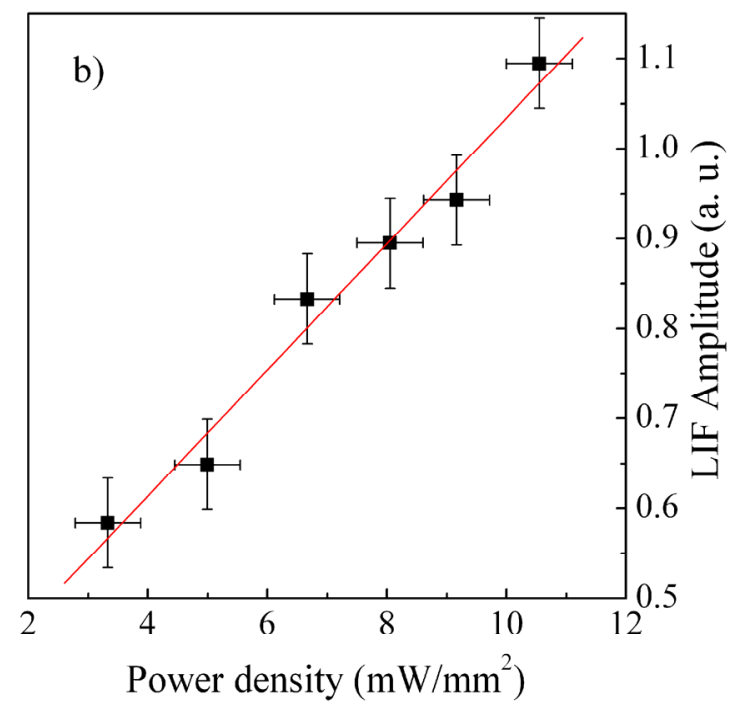

Power density $\left(\mathrm{mW} / \mathrm{mm}^{2}\right)$

Figure 4.22. a) The relative intensity of the AOM transmitted light in the 1st diffraction order versus Bragg angle misalignment; b) LIF signal amplitude versus injected laser power density. 


\subsubsection{LIF at Oblique Incidence}

Determination of a vdf measurement becomes complicated when, due to the geometry of the experiment, the laser has to be injected at an oblique angle relative to that magnetic field axis. In three dimensions, for a laser injection path $\boldsymbol{k}_{\boldsymbol{L}}$ at an angle $\alpha$ relative to the $z$ axis and an angle $\beta$ relative to the $x$ axis (see Figure 4.23 a), the measured vdf can be expressed as

$$
\begin{aligned}
& f\left(v_{\alpha \beta}, \alpha, \beta\right)= \\
& \iiint_{\text {band }} f\left(v_{x}, v_{y}, v_{z}\right) \delta\left[\left(v_{y} \sin \alpha+v_{z} \cos \alpha\right) \sin \beta+v_{x} \cos \beta-v_{\alpha \beta}\right] d v_{x} d v_{y} d v_{z},
\end{aligned}
$$

where $v_{\alpha \beta}$ is the velocity along $\boldsymbol{k}_{\boldsymbol{L}}, \delta()$ is the Dirac delta function, and the range (band) of integration is given by the Doppler resonance condition

$$
\left|\left(v_{y} \sin \alpha+v_{z} \cos \alpha\right) \sin \beta+v_{x} \cos \beta-\lambda \Delta \omega_{L} / 2 \pi\right|<\lambda \Delta \omega_{0} / 2 \pi,
$$

where $\lambda, \Delta \omega_{L} / 2 \pi$ and $\Delta \omega_{0} / 2 \pi$ are the wavelength of the transition in the rest frame, the detuning of the laser frequency from the Doppler shifted line center, and the homogenous linewidth of the absorption line, respectively.

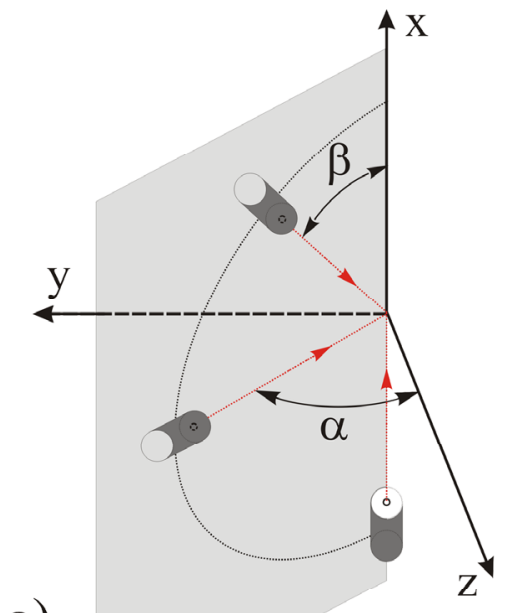

a)

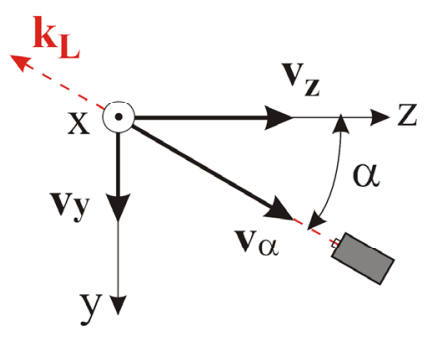

b)

Figure 4.23. LIF injection geometries: a) in a vertical plane $(\alpha, x)$ at different angles $\beta$ with respect to the $x$ axis for LIF tomography; $\mathrm{b}$ ) in a horizontal plane $(y, z)$ for obtaining $v_{y}$ and $v_{z}$. 
Eq. (4.53) is nothing else than the requirement that the optical pumping LIF process, excitation of the particle and the subsequent fluorescent emission, takes place only if the detuning from the Doppler shifted line center is less than the homogenous line width of the absorption line. Since $f\left(v_{\alpha \beta_{3}}, \beta\right)$ is a convolution of $f\left(v_{x}\right), f\left(v_{y}\right)$ and $f\left(v_{z}\right)$, determination of average particle velocities and temperatures in each $x y z$ direction is more difficult. Optical tomography is the only technique that can provide two- or three-dimensional ivdfs. However, under the cylindrical symmetry usually encountered in many laboratory plasmas, the problem simplifies. For laser injection oblique to an axis in two dimensions, for example, when $\boldsymbol{k}_{\boldsymbol{L}}$ lies in the $(y, z)$ plane at an angle $\alpha$ with respect to the $z$ axis as shown in Figure 4.23 b), Eq. (4.52) reduces to

$$
f\left(v_{\alpha}, \alpha\right)=\iint_{\text {linewidth }} f\left(v_{y}, v_{z}\right) \delta\left(v_{y} \sin \alpha+v_{z} \cos \alpha-v_{\alpha}\right) d v_{y} d v_{z} .
$$

The problem of finding the velocity distributions along the axial $(z)$ and radial $(r)$ directions is solvable by injecting the laser along another direction (preferably along $x$ or $y$ to directly measure $f\left(v_{x}\right)$ or $\left.f\left(v_{y}\right)\right)$, and then with cylindrical symmetry, i.e., $f\left(v_{x}\right)=f\left(v_{y}\right)=$ $f\left(v_{r}\right), f\left(v_{\infty} \alpha\right)$ can be deconvolved to find $f\left(v_{z}\right)$. Because a Gaussian Doppler broadened particle velocity component distribution will remain Gaussian for any injection angle, ${ }^{36}$ the radial and axial temperatures can also be determined if $f\left(v_{\alpha} \alpha\right)$ and $f\left(v_{r}\right)$ are measured. For the specific case of a cylindrical plasma column with an axial magnetic field, an expression for the component, parallel and perpendicular, ion temperatures can be derived by introducing a general anisotropic bi-Maxwellian distribution ${ }^{37}$

$$
f\left(v_{r}, v_{z}\right)==n\left(\frac{m}{2 \pi k_{B}}\right)^{3 / 2} \frac{1}{T_{r} T_{z}^{1 / 2}} \exp \left[-\frac{m}{2 k_{B}}\left(\frac{v_{r}^{2}}{T_{r}}+\frac{v_{z}^{2}}{T_{z}}\right)\right] .
$$

Ion temperatures along the $r$ and $z$ axes are obtained from the weighted averages of the radial and axial kinetic energies

$$
\frac{k_{B} T_{r}}{m}=\frac{1}{2 n}\left\langle v_{r}^{2}\right\rangle=\frac{\pi}{n} \int_{-\infty}^{\infty} \int_{0}^{\infty} v_{r}^{3} f\left(v_{r}, v_{z}\right) d v_{r} d v_{z}
$$

and

$$
\frac{k_{B} T_{z}}{m}=\frac{1}{n}\left\langle v_{z}^{2}\right\rangle=\frac{2 \pi}{n} \int_{-\infty}^{\infty} \int_{0}^{\infty} v_{z}^{2} v_{r} f\left(v_{r}, v_{z}\right) d v_{r} d v_{z},
$$

with the normalization condition 


$$
n=\int_{-\infty}^{\infty} \int_{0}^{\infty} \int_{0}^{2 \pi} f\left(v_{r}, v_{z}\right) v_{r} d v_{r} d v_{z} d \varphi=2 \pi \int_{-\infty}^{\infty} \int_{0}^{\infty} f\left(v_{r}, v_{z}\right) v_{r} d v_{r} d v_{z} .
$$

In this formulation, the total ion temperature is then

$$
T=\left(T_{z}+2 T_{r}\right) / 3
$$

To relate these temperature components to the "temperature" of the distribution along the $\alpha$ direction (see Figure 4.23), we note that by choosing the measurement location to be close to the $z$ axis, the azimuthal velocity component is almost zero and the LIF measured velocity distribution along $\alpha$ is effectively only a projection of the $v_{z}$ and $v_{y}=v_{r} / \sqrt{2}$ velocity components along the laser direction:

$$
-v_{\alpha}=v_{z} \cos \alpha+\left(v_{r} / \sqrt{2}\right) \sin \alpha .
$$

Then,

$$
\frac{k_{B} T_{\alpha}}{m}=\frac{1}{n}\left\langle v_{\alpha}^{2}\right\rangle=\frac{\pi}{n}\left[\begin{array}{l}
2 \cos ^{2} \alpha \int_{-\infty}^{\infty} \int_{0}^{\infty} v_{z}^{2} v_{r} f\left(v_{r}, v_{z}\right) d v_{r} d v_{z}+ \\
\sin ^{2} \alpha \int_{-\infty}^{\infty} \int_{0}^{\infty} v_{r}^{3} f\left(v_{r}, v_{z}\right) d v_{r} d v_{z}+2 \sqrt{2} \sin \alpha \cos \alpha \int_{-\infty}^{\infty} \int_{0}^{\infty} v_{z} v_{r}^{2} f\left(v_{r}, v_{z}\right) d v_{r} d v_{z}
\end{array}\right] .
$$

Since in the third double integral, $\int_{-\infty}^{\infty} v_{z} \exp \left(-m v_{z}^{2} / 2 k_{B} T_{z}\right) d v_{z}$, vanishes, the " $\alpha$ " ion temperature in terms of the axial and radial temperatures is:

$$
T_{\alpha}=T_{z} \cos ^{2} \alpha+T_{r} \sin ^{2} \alpha .
$$

Thus, two LIF measurements (along the $\alpha$ and $r$ directions) are enough to determine the axial and radial ion temperatures. Note that for $\alpha=0$ or $\pi / 2, T_{\alpha}$ reduces to parallel (axial) or perpendicular (radial) temperature.

\subsubsection{LIF Tomography}

If a full 2D or $3 \mathrm{D}$ velocity distribution is needed, the complete vdf can be found via a more sophisticated LIF technique developed by McWilliams and co-workers -

optical tomography. ${ }^{38,39}$ For laser injection at an angle $\beta$ with respect to the $x$ axis (see 
Figure 4.23 a), the 1D projection of the velocity distribution along the laser propagation direction $\left(\boldsymbol{k}_{\boldsymbol{L}}\right)$ is

$$
f\left(v_{\beta}, \beta\right)=\iint_{\text {linewidth }} f\left(v_{\alpha}, v_{x}\right) \delta\left(v_{\alpha} \sin \beta+v_{x} \cos \beta-v_{\beta}\right) d v_{\alpha} d v_{x} .
$$

Since the integrand vanishes for frequencies outside of the absorption linewidth, the limits of the integral in Eq. (4.63) can be set to $\pm \infty$ without effect. A collection of such 1D projections taken at different injection angles $\beta$ in the plane $(\alpha, x)$ is equivalent to the continuous 2D Radon transform, the mathematical basis for medical tomography. With a collection of such 1D ivdfs, the complete reconstruction of the 2D ivdf is obtained by inverting the 2D Radon transform with a filtered back-projection algorithm. ${ }^{40}$ To eliminate blurring in the final reconstructed ivdf tomograph, each projection is convolved with a filter function $\varphi(v)$

$$
\Phi\left(v_{\beta}, \beta\right)=\int_{-\infty}^{+\infty} f\left(v_{\beta}, \beta\right) \varphi\left(v_{\beta}-v\right) d v .
$$

The choice of filter function is a trade-off between the Nyquist limit (the highest velocity space Fourier harmonic at which the smearing of the reconstructed ivdf is negligible) and noise in the final reconstructed ivdf. From the Fourier slice theorem, it is known that 1D Fourier transform of the projection function $\Phi\left(v_{\beta}, \beta\right)$ with respect to $v_{\beta}$ is equal to the central slice at angle $\beta$ of the two-dimensional Fourier transform of $f\left(v_{\infty}, v_{x}\right)$. Thus, the 2D inverse Fourier transform provides a two-dimensional velocity space slice [the $\left(v_{\alpha}, v_{x}\right)$ plane] of the 3D ivdf

$$
f\left(v_{\alpha}, v_{x}\right)=\frac{1}{4 \pi^{2}} \int_{-\infty}^{+\infty} \int_{0}^{\pi} \mathscr{F}\left(\tilde{v}_{\beta}, \beta\right) \exp \left[j \tilde{v}_{\beta}\left(v_{\alpha} \sin \beta+v_{x} \cos \beta\right)\right]\left|\tilde{v}_{\beta}\right| d \tilde{v}_{\beta} d \beta,
$$

where $\mathscr{J}\left(\tilde{v}_{\beta}, \beta\right)$ is the Fourier transform of the filter function convolved projection $\Phi$, $\tilde{v}_{\beta}$ is the conjugate variable to $v_{\beta}$, and $j=\sqrt{-1}$. Note that according to Eq. (4.65), the angular range of the measurements need only span $\pi$ radians to obtain the full 2D ivdf, i.e., projections in opposite direction give same information. Since a key measurement in this work is plasma flow, this feature allows the choice of tomographic probe orientations that do not block the plasma flow, yet still provide full tomographic data. 
Actual measurements consist of only a finite number of laser injection angles $(I)$ with a uniform sampling $\Delta \beta$ so that $I \Delta \beta=\pi$. Thus, Eq. (4.65) must be approximated with a discrete series ${ }^{41}$

$$
f\left(v_{\alpha}, v_{x}\right) \approx \frac{1}{2 I} \sum_{i=1}^{I} \mathscr{F}^{-1}\left\{\left[\mathscr{F}\left(\Phi_{i}\left(v_{\alpha} \sin \frac{i-1}{I} \pi+v_{x} \cos \frac{i-1}{I} \pi\right)\right)\right]\left|\tilde{v}_{i}\right|\right\} .
$$

The full 3D vdf could be obtained with a three-dimensional Radon transform ${ }^{42}$ if a second plane of projections were available [(y,z) for the geometry of Figure 4.23 a)]. However, given the difficulty of representing and interpreting four-dimensional structures, the 3D method is of less interest for vdf analysis.

\subsection{Scanning Internal Probe for LIF Tomography}

The need to develop a theoretical framework for LIF investigations at oblique (to the $z$-axis) incidence arose from the desire to characterize the ion beam resulting from acceleration by the electric double layer (EDL) at multiple spatial locations in the LEIA chamber. The LIF measurements in LEIA are obtained with internal scanning probe capable of spatially resolved measurements throughout a horizontal $(y, z)$ cross-section of the expansion region between HELIX and LEIA (see Figure 4.24).

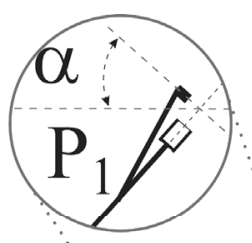

LEIA magnèts

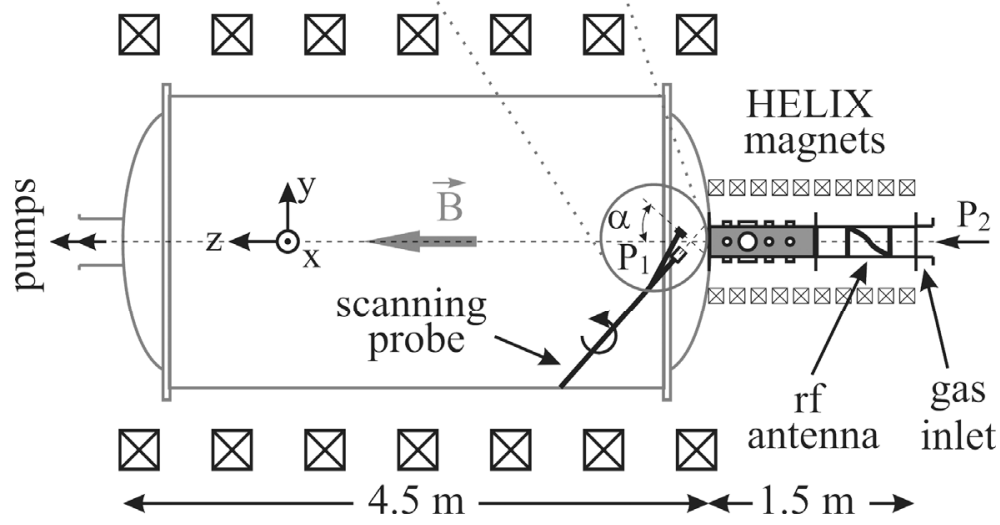

Figure 4.24. Horizontal cross-section of the HELIX-LEIA helicon source-diffusion chamber system and an expanded view of the injection geometry corresponding to Figure $4.23 \mathrm{~b}$ ) 


\section{Chapter 4: Plasma Diagnostics}

The probe consists of a $183 \mathrm{~cm}$ long shaft terminated in a diagnostics complement comprised of LIF injection and collection optics, a rf compensated cylindrical Langmuir probe, and a 3D magnetic sense coil array (see Figure 4.25). The other end of the probe shaft passes through a custom bearing and then through a feedthrough flange mounted on a welded bellows coupled to the LEIA chamber. Motions along the $y$-axis (40 $\mathrm{cm}$ range) and $z$-axis (100 $\mathrm{cm}$ range) are accomplished with two computer-driven VELMEX ${ }^{\mathrm{TM}}$ stepping motor assemblies that control the insertion depth of the probe and the tilt angle between the probe and the chamber axis. A third VELMEX ${ }^{\mathrm{TM}}$ stepping motor spins the probe shaft (and implicitly the laser injection direction) around its axis to switch between parallel and perpendicular (with respect to the magnetic field) LIF measurements and for optical tomography. In this way, the LIF investigated plasma volume (the intersection between the laser beam and the collection optics field of view) remains the same for all injection angles. For complete diagnosis of the expanding plasma, the probe is designed to simultaneously measure the electron temperature, the electron density, the plasma potential, the magnetic fluctuation spectrum in three dimensions, and the onedimensional ion velocity distribution (ivdf) function. Through a fiber-fiber vacuum feedthrough the incident laser light is coupled into an internal $200 \mu \mathrm{m}$ fused silica fiber that runs along the inside of the probe shaft. The injection fiber is terminated with a $6 \mathrm{~mm}$ collimating lens to create a weakly divergent beam that reflects from a plane mirror and passes $5 \mathrm{~cm}$ in front of the collection optics (see Figure 4.25). Between the focusing lens and the collection fiber, a series of light baffles (shown in Figure 4.25 b) prevent off-axis rays from passing through the lenses and into the collection fiber. Because the plasma emits strongly at the fluorescence wavelength, reduction of background light is critical for improving the measurement signal-to-noise ratio. The collected fluorescence radiation is collimated inside the probe, passes through a standard fused silica window, a Dell Optics 1-nm band pass interference filter (centered around $461 \mathrm{~nm}$ for $\mathrm{Ar}^{+} \mathrm{LIF}$ ) and into the PMT. The parallel ivdf can be measured either "actively", by injecting the laser from the probe head or "passively", by injecting the laser parallel along the axis of the system from the HELIX end (injection $\mathrm{P}_{2}$ in Figure 4.24) and only using the probe collection optics. By measuring the parallel ivdf with both laser injection options, the corrections for oblique laser LIF are easily checked. 


\section{Chapter 4: Plasma Diagnostics}

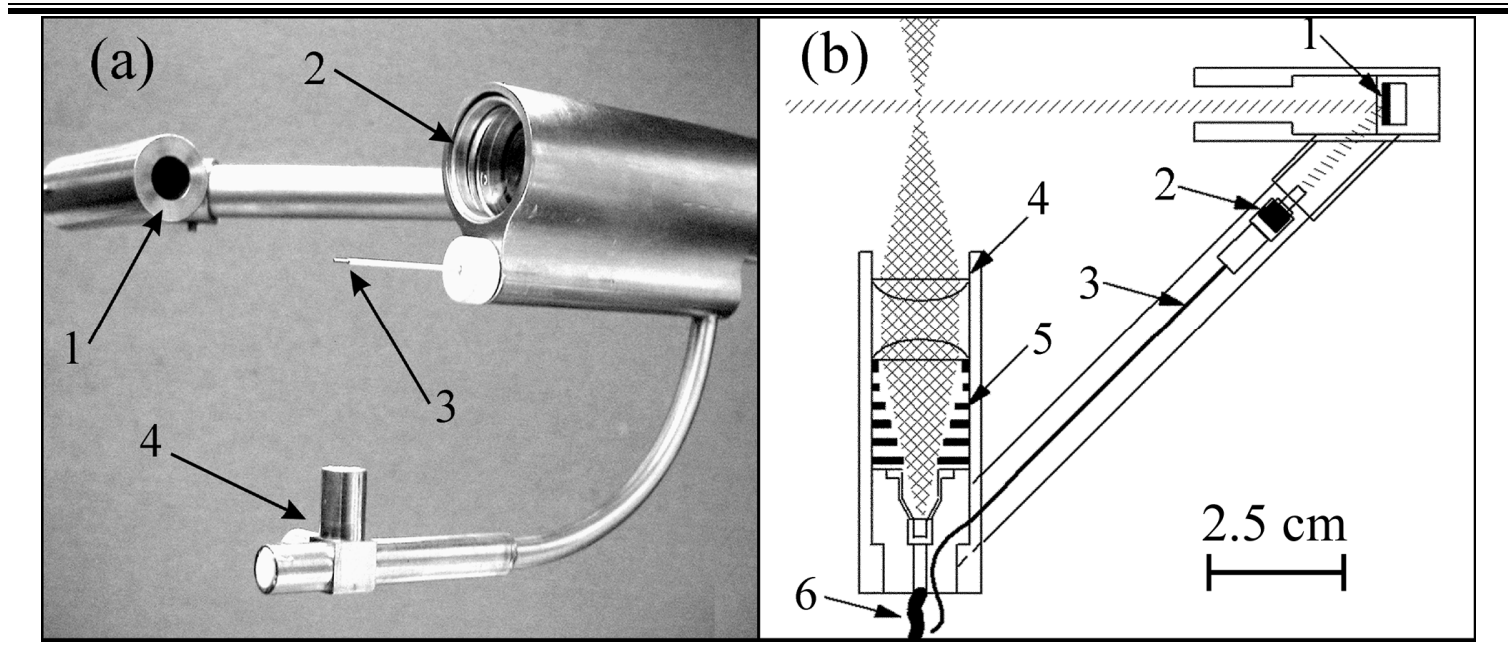

Figure 4.25. Scanning probe head diagnostic complement: (a) 1 - LIF injection optics; 2 - LIF collection optics; 3 - rf compensated Langmuir probe; 4 - 3D magnetic sense coil array. (b) 1-injection mirror; 2 collimating injection optic; 3 - injection fiber; 4 - collection lens; 5 - light baffles; 6 - collection fiber.

Due to the losses at each optical interface and in the fibers, only about $40 \%$ of the laser power is effectively injected into the plasma. The injected laser power density of $\sim 10$ $\mathrm{mW} / \mathrm{mm}^{2}$ ensures that the laser optical pumping is in a linear regime, i.e., the LIF signal is proportional to the laser intensity and LIF saturation effects are avoided. ${ }^{43,44}$ 


\section{Chapter 4 References}

${ }^{1}$ I. Langmuir, Phys. Rev. 28, 727 (1926)

${ }^{2}$ F. F. Chen, Mini-course on plasma diagnostics, IEEE-ICOPS meeting, Korea (2003)

${ }^{3}$ J. G. Lafambroise and J. Rubinstein, J. Phys. D: Appl. Phys. 19, 1900 (1976)

${ }^{4}$ I. H. Hutchinson, Principles of Plasma Diagnostics, Cambridge University Press, Cambridge (1987)

${ }^{5}$ K. H. Bai, H. Y. Chang, and H. S. Uhm, Appl. Phys. Lett. 79, 1596 (2001)

${ }^{6} \mathrm{X}$. Sun, PhD Dissertation, West Virginia University (2005)

${ }^{7}$ C. Biloiu, E. Scime, X. Sun, and B. McGeehan, Rev. Sci. Instrum. 75, 4296 (2004)

${ }^{8}$ M. J. Druyvesteyn, Z. Phys. 64, 781 (1930)

${ }^{9}$ V. A. Godyak, R. B. Piejak, and B. M. Alexandrovich, J. Appl. Phys. 73, 3657 (1993)

${ }^{10}$ K. Giapis, N. Sadeghi, J. Margot, R. Gottscho, and T. C. John Lee, J.Appl. Phys. 73, 7188 (1993)

${ }^{11}$ M. V. Malyshev and V. M. Donnelly, J. Vac. Sci. Technol. A 15, 550 (1997)

${ }^{12}$ K. L. Junck and W. D. Getty, J. Vac. Sci. Technol. A 12, 2767 (1994)

${ }^{13}$ C. Biloiu, X. Sun, Z. Harvey, and E. Scime, J. Appl. Phys. 101, 073303 (2007)

${ }^{14}$ NIST atomic spectral lines database, www.nist.gov

${ }^{15}$ R. F. Boivin, Internal Report PLP-039, West Virginia University (2000)

${ }^{16}$ P. Schef, A. Derkatch, P. Lundin, S. Mannervik, L. O. Norlin, D. Rostohar, P. Koyen, and E. Biemont, Eur. Phys. J. D 94, 195 (2004)

${ }^{17}$ V. Vujnovic and W. L. Wiese, J. Phys. Chem. Ref. Data 21, 919 (1992)

${ }^{18}$ R. F. Boivin, Internal Report PLP-050, West Virginia University (2001)

${ }^{19}$ R. F. Boivin, Zeeman splitting for LIF transitions and deconvolution technique to extract the ion temperature, EPAPS Deposited Document E-PHPAEN-10-003306 (2003)

${ }^{20}$ L. Broström, S. Mannervik, A. Passian, and G. Sundström, Phys. Rev. A 49, 3333 (1994)

${ }^{21}$ E. Browne, Table of Isotopes, C. M. Lederer and V. S. Shirley, 7-th ed., Wiley, (1978)

${ }^{22}$ C. R. Bingham, M. L. Gaillard, D. J. Pegg, H. K. Carter, R. L. Mlekodaj, J. D. Cole, and P. M. Griffin, Nuclear Instruments and Methods 202, 147 (1982)

${ }^{23}$ G. Borghs, P. De Bisschop, R. E. Silerans, M. Van Hove, and J. M. Van den Cruyce, Zeitschrift fur Physik A 299, 11 (1981)

${ }^{24}$ G. Herzberg, Atomic Spectra and Atomic Structure, Dover Publications (1944)

${ }^{25}$ T. B. Smith, PhD Dissertation, The University of Michigan (2003)

${ }^{26}$ L. Broström, A. Kastberg, J. Lidberg, and S. Mannervik, Phys. Rev. A 53, 109 (1996)

${ }^{27}$ H. E. White, Introduction to Atomic Spectra, McGraw-Bill Book Company (1934)

${ }^{28}$ R. J. Cedolin, PhD Dissertation, Stanford University (1997)

${ }^{29}$ G. Bachet, L. Cherigier, C. Arnas-Capeau, F. Doveil and R. A. Stern, J. Phys. III France 6, 1157 (1996)

${ }^{30}$ B. Pelissier and N. Sadeghi, Rev. Sci. Instrum. 67, 3405 (1996)

${ }^{31}$ G. Bachet, F. Skiff, M. Dindelegan, F. Doveil, and R. A. Stern, Phys. Rev. Lett. 80, 3260 (1998) 
${ }^{32}$ E. Scime, C. Biloiu, C. Compton, F. Doss, D. Venture, J. Heard, E. Choueiri and R. Spektor, Rev. Sci. Instrum. 76, 026107 (2005)

${ }^{33}$ C. Biloiu, X. Sun, E. Choueri, F. Doss, E. Scime, J. Heard, R. Spektor and D. Ventura Plasma Sources Sci. Technol. 14766 (2005)

${ }^{34}$ C. Davis, Lasers and Electro-Optics - Fundamentals and Engineering, Cambridge University Press, Cambridge, UK (2000), pp.495

${ }^{35}$ www.ISOMET.com, All about Bragg Angle errors in AO Modulators \& Deflectors - Application Note IM 1022

${ }^{36}$ W. M. Ruyten and D. Keefer, AIAA Jour. 31, 2083 (1993)

${ }^{37}$ A. R. Barakat and R. W. Schunk, J. Phys. D: Appl. Phys. 14, 421 (1981)

${ }^{38}$ R. Koslover and R. McWilliams, Rev. Sci. Instrum. 57, 2441 (1986)

${ }^{39}$ M. Zintl and R. McWilliams, Rev. Sci. Instrum. 65, 2574 (1994)

${ }^{40}$ A. G. Ramm and A. I. Katsevich, The Radon Transform and Local Tomography, CRC, Boca Raton, FL (1996)

${ }^{41}$ D. C. Zimmerman, R. McWilliams and D. A. Edrich, Plasma Sources Sci. Technol. 14, 581 (2005)

${ }^{42}$ A. V. Bronnikov, J. Opt. Soc. Am. A 19, 472 (2002)

${ }^{43}$ M. J. Goecker and J. Goree, J. Vac. Sci. Technol. A 7, 977 (1989)

${ }^{44}$ F. Skiff and J. Bollinger, Phys. Plasmas 11, 2972 (2004) 


\section{Chapter 5: Electron and Ion Energy Distribution Functions in Two-Ion Species, Helicon Plasmas}

\subsection{Effects of Gas Composition on Plasma Density and Electron Temperature in an Ar-Xe Helicon Plasma}

For mixed gas plasmas with a single, thermal, electron population, the classic Langmuir method of determining the electron temperature, Eq. (4.4), is still appropriate. However, it is impossible to use the standard Langmuir probe analysis to accurately determine the plasma density from the ion saturation region of the probe characteristic since the relative ion densities and their Bohm speeds at the plasma-sheath interface are unknown. As shown in the previous chapter, another approach is to first determine the electron energy distribution function (eedf) and then from the eedf calculate the electron temperature and plasma density. The experimental electron energy probability functions (eepf) presented in this work were obtained from Langmuir probe measurements as described in Chapter 4. Typical Langmuir I-V probe data acquisition consisted of averaging 10-20 I-V traces (depending on the observed fluctuation levels), applying a smoothing function with Origin ${ }^{\mathrm{TM}}$ software, and then taking numerical derivatives (single derivative for planar probe and double derivative for cylindrical probe measurements) to obtain the electron energy distribution function (eedf) for planar probe measurements and the electron energy probability function (eepf) for cylindrical probe measurements.

\subsubsection{Electron Energy Distribution Function (EEDF) in the Plasma Source (HELIX)}

To investigate the effects of the gas composition on plasma parameters, electron energy probability functions (eepf) were obtained at the middle of HELIX, $\sim 20 \mathrm{~cm}$ downstream from the antenna, for different $\mathrm{Ar} / \mathrm{Xe}$ ratios and roughly constant total pressure. The other source parameters were: a gas flow rate of $10 \mathrm{sccm}$ (standard cubic centimeter per minute), which yielded a fill pressure of $1.3 \mathrm{mTorr}$ for Ar and $1.5 \mathrm{mTorr}$ for Xe; a HELIX magnetic field strength of $700 \mathrm{G}$, a LEIA magnetic field strength of 10 
$\mathrm{G}$, an input power of $750 \mathrm{~W}$, and a rf driving frequency $9.5 \mathrm{MHz}$. Ideally, control of the relative gas composition is accomplished through measurement and control of partial gas pressures. However, the only adjustable gas parameter for these experiments was the mass flow rate of each species. Therefore, $\mathrm{Ar} / \mathrm{Xe}$ ratio was varied by adjusting $\mathrm{Ar}$ and $\mathrm{Xe}$ individual mass flow rates while holding the total flow rate constant at $10 \mathrm{sccm}$. The estimated error in gas composition determination due to the slight difference in actual Ar and Xe gas pressures $(0.2 \mathrm{mTorr}$ at $10 \mathrm{sccm})$ is less than $7 \%$. Figure 5.1 shows the electron temperature and electron density from experimentally obtained eepfs as a function of gas composition in mixed Ar-Xe helicon plasma. Numerical integrations of the eepfs give an effective electron temperature of $6.5 \mathrm{eV}$ and an electron density of $1.07 \times 10^{11} \mathrm{~cm}^{-3}$ for pure Ar plasma. For pure Xe plasma, an effective electron temperature of $3.8 \mathrm{eV}$ and an electron density of $1.25 \times 10^{11} \mathrm{~cm}^{-3}$ were obtained. The increase in plasma density with increasing xenon fraction is roughly linear. Even though xenon and argon are both noble gasses, xenon has a significantly lower excitation threshold energy (8.31 eV versus $11.54 \mathrm{eV}$ for argon), a lower ionization threshold energy (12.13 eV versus $15.76 \mathrm{eV}$ for argon), and a larger peak ionization cross-section $\left(5.2 \times 10^{-16} \mathrm{~cm}^{2}\right.$ versus $2.8 \times 10^{-16} \mathrm{~cm}^{2}$ for argon). These differences are responsible for the dramatic changes in plasma properties that occur with increasing xenon fraction.
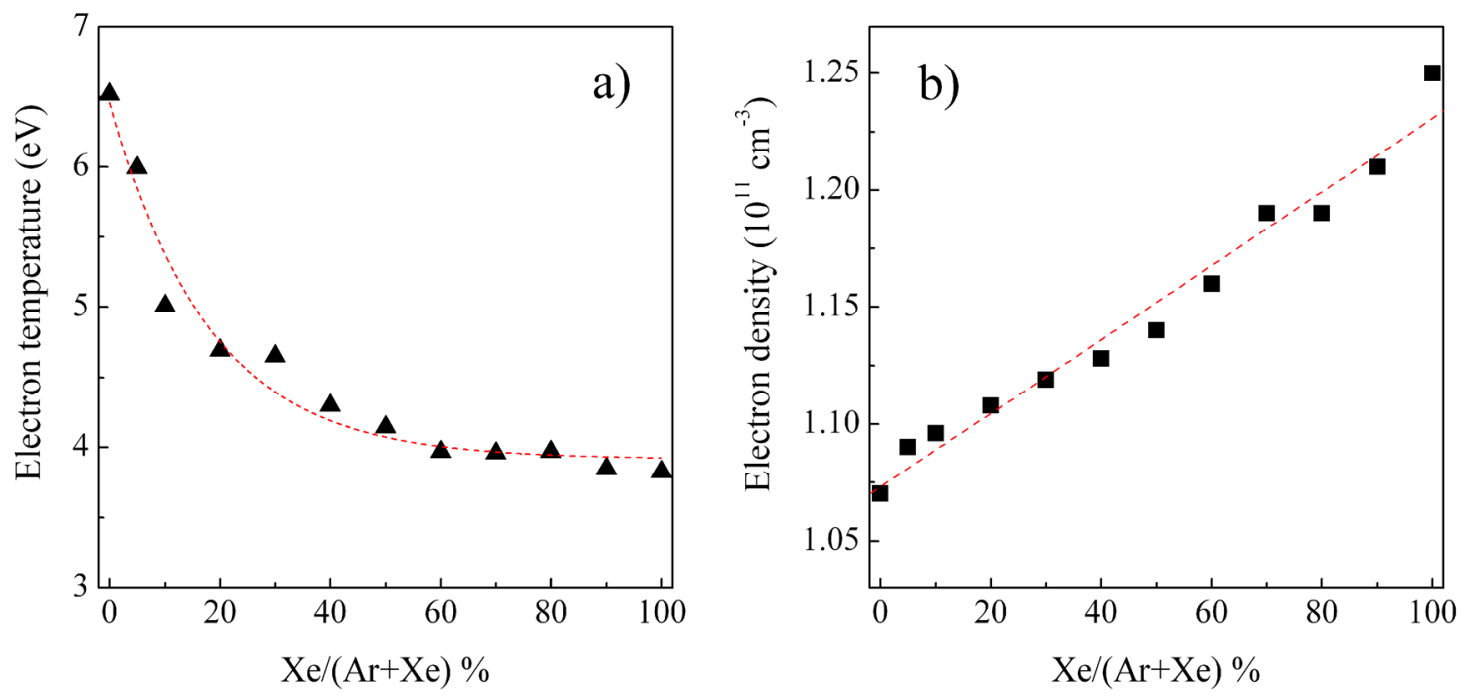

Figure 5.1. a) Effective electron temperature and b) electron density obtained from experimentally obtained eepfs at $r=0$ in HELIX, $20 \mathrm{~cm}$ downstream from the antenna, as a function of $\mathrm{Ar} / \mathrm{Xe}$ composition. The dashed lines are exponential and linear fits to the experimental data. Source parameters: $\mathrm{P}_{\mathrm{rf}}=750 \mathrm{~W}, B_{H}=700 \mathrm{G}, B_{L}=10 \mathrm{G}, f=9.5 \mathrm{MHz}$, and $F=10 \mathrm{sccm}$ 
Addition of xenon to argon dramatically lowers the effective electron temperature. The decrease in effective electron temperature is exponential with increasing xenon fraction and most of the drop in electron temperature $(\sim 2 \mathrm{eV})$ occurs as the xenon fraction increases from 0 to $20 \%$. The effective electron temperature values obtained from eepf integration are in very good agreement with the corresponding electron temperature values obtained from the slopes of the eepfs: $6.5 \mathrm{eV}$ and $3.6 \mathrm{eV}$ for pure argon and xenon plasmas, respectively.

\subsubsection{Electron Energy Distribution Function (EEDF) in the Expansion Chamber (LEIA)}

For the same operating conditions as for the mixed gas experiments described previously, planar Langmuir probe investigations were carried out on the axis of the expansion chamber axis, $20 \mathrm{~cm}$ downstream of the HELIX-LEIA junction. The eedfs for different $\mathrm{Ar} / \mathrm{Xe}$ ratios were computed by using Eq. (4.12). Similar as plasmas occurred in the helicon source, addition of xenon to an argon plasma dramatically changes the plasma properties.

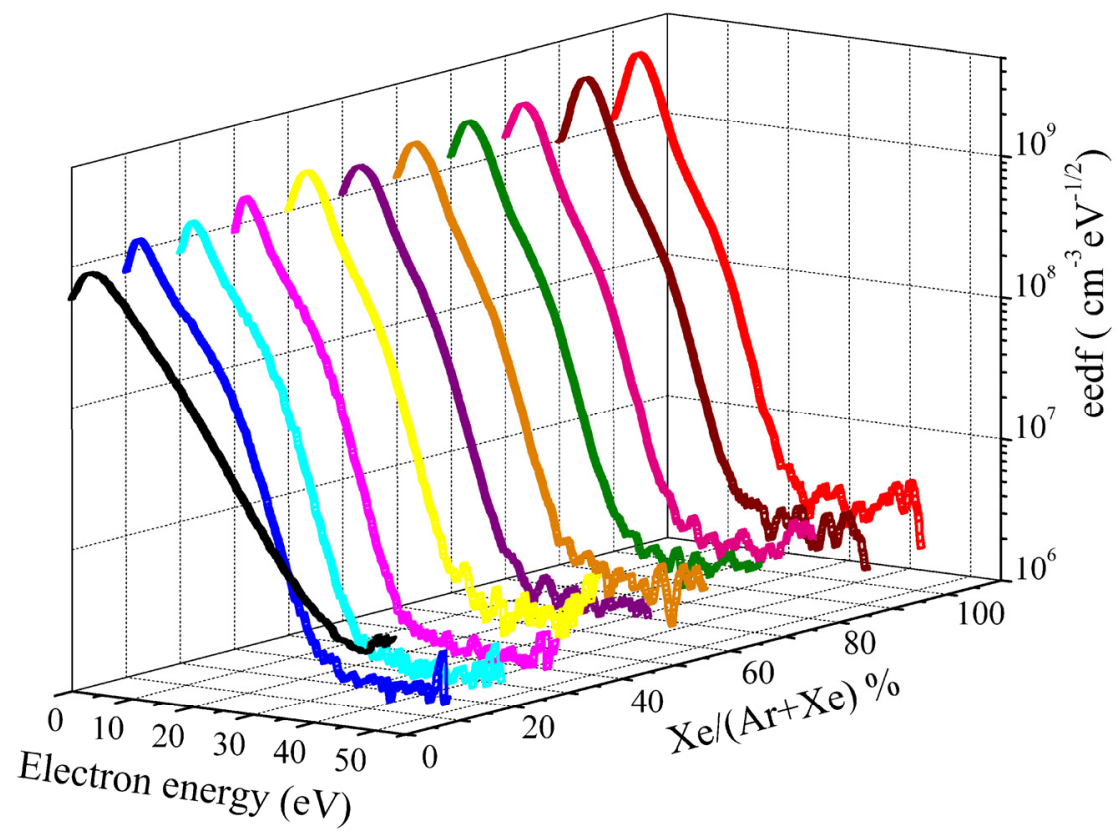

Figure 5.2. A family of eedfs in Ar-Xe helicon plasma measured on the expansion chamber axis, $20 \mathrm{~cm}$ downstream the HELIX-LEIA junction. Plasma parameters were: $\mathrm{P}_{\mathrm{rf}}=750 \mathrm{~W}, f=9.5 \mathrm{MHz}, F=10 \mathrm{sccm}$, $B_{H}=700 \mathrm{G}$, and $B_{L}=10 \mathrm{G}$. 
Shown in Figure 5.2 is a family of eedfs obtained for different xenon fractions. With increasing xenon fraction, the density increases (note that the eedf amplitudes span three orders of magnitude) and there is an increase in the low electron energy population with a simultaneous reduction in the high energy tail of the distribution. The significant change in the eedf is more easily seen in the two eedfs shown in Figure 5.3. In the pure argon plasma, the eedf is a single Maxwellian (straight line on the semi logarithmic plot). However, for the pure xenon plasma, the eedf is better fit with a Druyvesteyn distribution (convex curve). Consistent with the differences in excitation energies and ionization potentials, the breakpoint in the high energy eedf tail (the energy at which the eedf amplitude equals the noise level) decrease by $\sim 15 \mathrm{eV}$ for pure xenon plasma compared to pure argon. The transition from a single Maxwellian distribution to a Druyvesteyn distribution with increasing of the xenon percentage is not gradual. Once the xenon fraction reaches $10 \%$, the eedf is Druyvesteyn-like than Maxwellian-like.
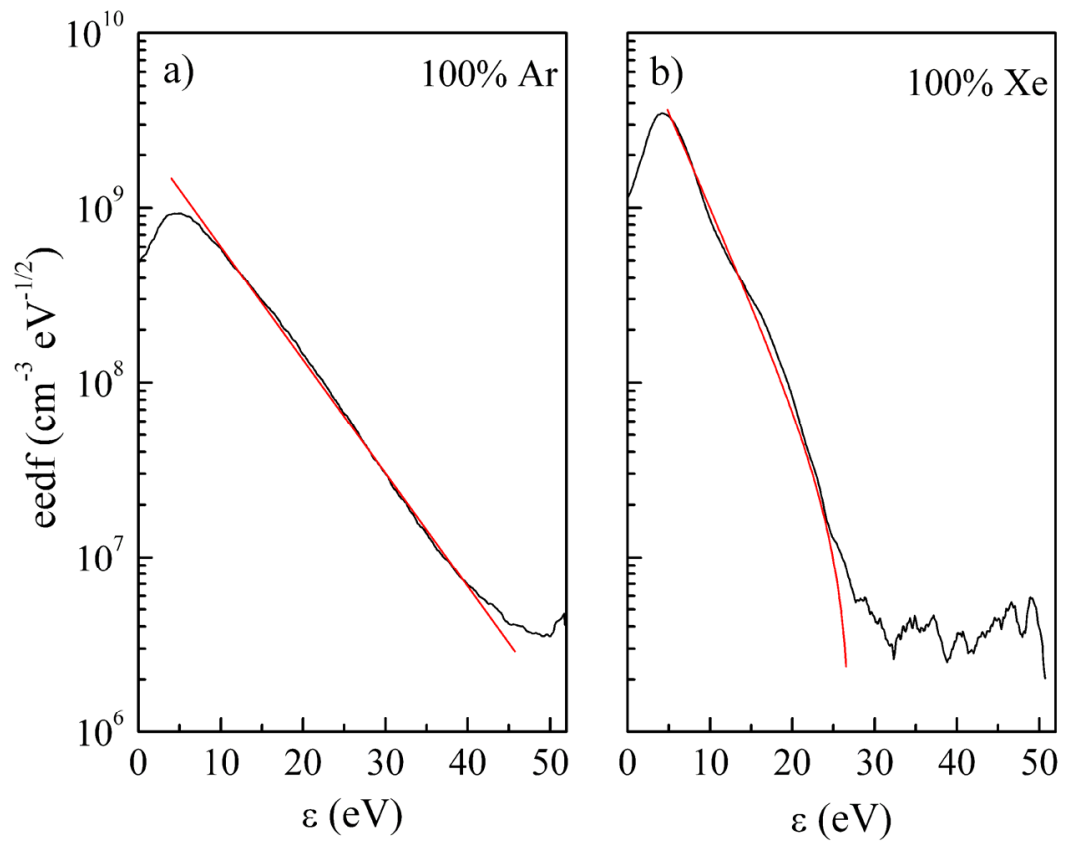

Figure 5.3. The bounding cases for the family of eedfs shown in Figure 5.2: a) eedf for pure argon b) and pure xenon.

The calculated effective electron temperature and electron density versus xenon fraction is shown in Figure 5.4. There is a systematic difference between the effective electron temperatures and electron densities calculated from integration of the eedf and the corresponding values calculated from the slope of the I-V characteristic on a semi 
logarithmic plot (Eq. (4.4) for electron temperature) and from the electron saturation region of the characteristic (from Eq. (4.2) for electron density). The effective electron temperature is roughly $1 \mathrm{eV}$ hotter than the corresponding values calculated from the I-V characteristic and the effective electron density is about 1.8 times smaller than the corresponding values calculated from the electron saturation current. These discrepancies are not surprising given the strongly non-Maxwellian nature of the measured eedfs. Differences as large as one order of magnitude in electron density have also been reported previously for eedf and ion saturation current methods. ${ }^{1}$ As pointed out by Sudit and Woods [1], the credible values are those determined from the eedf since they reflect the whole electron population. Although the term "electron temperature" has no physical meaning for distributions other than Maxwellian, we use it here for the Druyvesteyn distribution in the sense of average electron energy.

The trend in overall effective "electron temperature" dependence on xenon fraction is similar to what was observed in the source, i. e. an exponential decrease with increasing xenon fraction, from $\sim 7.2 \mathrm{eV}$ in pure argon to $4.7 \mathrm{eV}$ in pure xenon. Although almost two orders of magnitude lower, the electron density again increases linearly with increasing of xenon concentration, from $2 \times 10^{9} \mathrm{~cm}^{-3}$ in pure argon to $5 \times 10^{9} \mathrm{~cm}^{-3}$ in pure xenon.
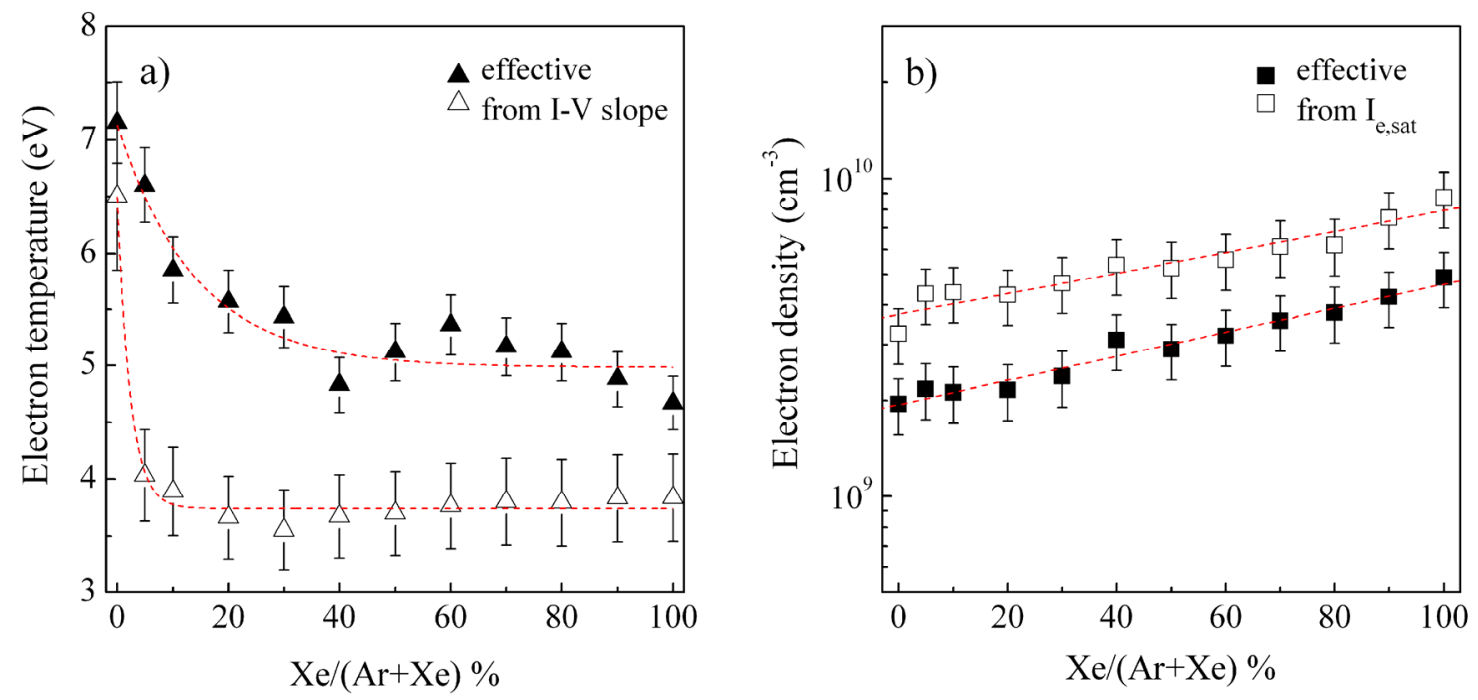

Figure 5.4. Electron temperature a) and electron density b) on the axis of LEIA, $20 \mathrm{~cm}$ downstream of the HELIX-LEIA junction versus xenon fraction; the filled symbols are effective values calculated from eedfs; the open symbols are electron temperatures determined from the slope of the I-V trace and electron densities determined from the electron saturation current. The dashed lines are exponential and linear fits for electron temperature and electron density, respectively. 

Species, Helicon Plasmas

\title{
5.2. Neutral and Ion densities in Ar-Xe Plasma from OES Observations
}

\author{
Combined optical emission spectroscopy (OES) and Langmuir probe
} measurements were used to quantitatively determine the neutral and ion densities in a helicon plasma that contains a mixture of argon and xenon. As discussed in detail in the previous chapter (section 4.2), for low density plasmas for which a steady-state Corona model is appropriate, electron impact excitation from only the atom or ion ground level can be assumed. A Corona model is appropriate for LEIA plasmas, but for the relatively high plasma densities attained in HELIX $\left(\geq 10^{11} \mathrm{~cm}^{-3}\right)$, secondary processes such as excitation from metastable levels cannot be neglected. Therefore, to accurately model the emission line relative intensities, the Corona model has to be extended to include excitation from metastable levels as well as the ground state.

An energy level diagram for both argon and xenon neutrals is shown in Figure 5.5. Argon and xenon atoms may be excited by electron collisions from their ground states to the Paschen $2 p$ levels. These excited levels decay on a short timescale $(\sim 20 \mathrm{~ns})$ to one of four levels (in Paschen notation, the short lived $1 \mathrm{~s}_{2}$ and $1 \mathrm{~s}_{4}$, and the metastable $1 \mathrm{~s}_{3}$ and $1 \mathrm{~s}_{5}$ states) and emit photons in the near-infrared region. Alternatively, electron impact excitation can occur from the ground states to levels above the $2 \mathrm{p}$ manifold. These levels also decay on a short time scale, accompanied by emission of a vacuum ultraviolet (VUV) photon when decaying to the ground state, or longer wavelength photons when decaying to the $2 p$ or higher-lying levels. Decays to levels above the $2 p$ levels are followed by a cascade that increases the intensity of emission from the $2 p$ levels. Thus, when trying to model the intensity of spectral lines originating from the $2 p$ levels, the cascade pathways must be included. Fortunately, cascade effects are automatically included if measured optical cross-sections are used in the rate coefficients calculations instead of the theoretical cross-section values. ${ }^{2}$ In addition to the excitation from the ground state, electron impact excitation from the metastable levels $1 \mathrm{~s}_{3}$ and $1 \mathrm{~s}_{5}$ can populate the argon and xenon Paschen $2 \mathrm{p}$ levels for higher plasma densities. The peak cross-section values for direct excitation from the metastable levels to the $2 p$ levels are much larger than those for excitation from the ground state (15 to 700 times larger). Therefore, this mechanism must also be included in the particle balance equations. The 
other two low-lying $1 \mathrm{~s}$ Paschen states, $1 \mathrm{~s}_{2}$ and $1 \mathrm{~s}_{4}$, have short radiative lifetimes and decay to the ground state. Therefore their population is much lower than the $1 \mathrm{~s}_{3}$ and $1 \mathrm{~s}_{5}$ metastable states and consequently their contribution to the $2 \mathrm{p}$ levels population through electron impact excitation is negligible.

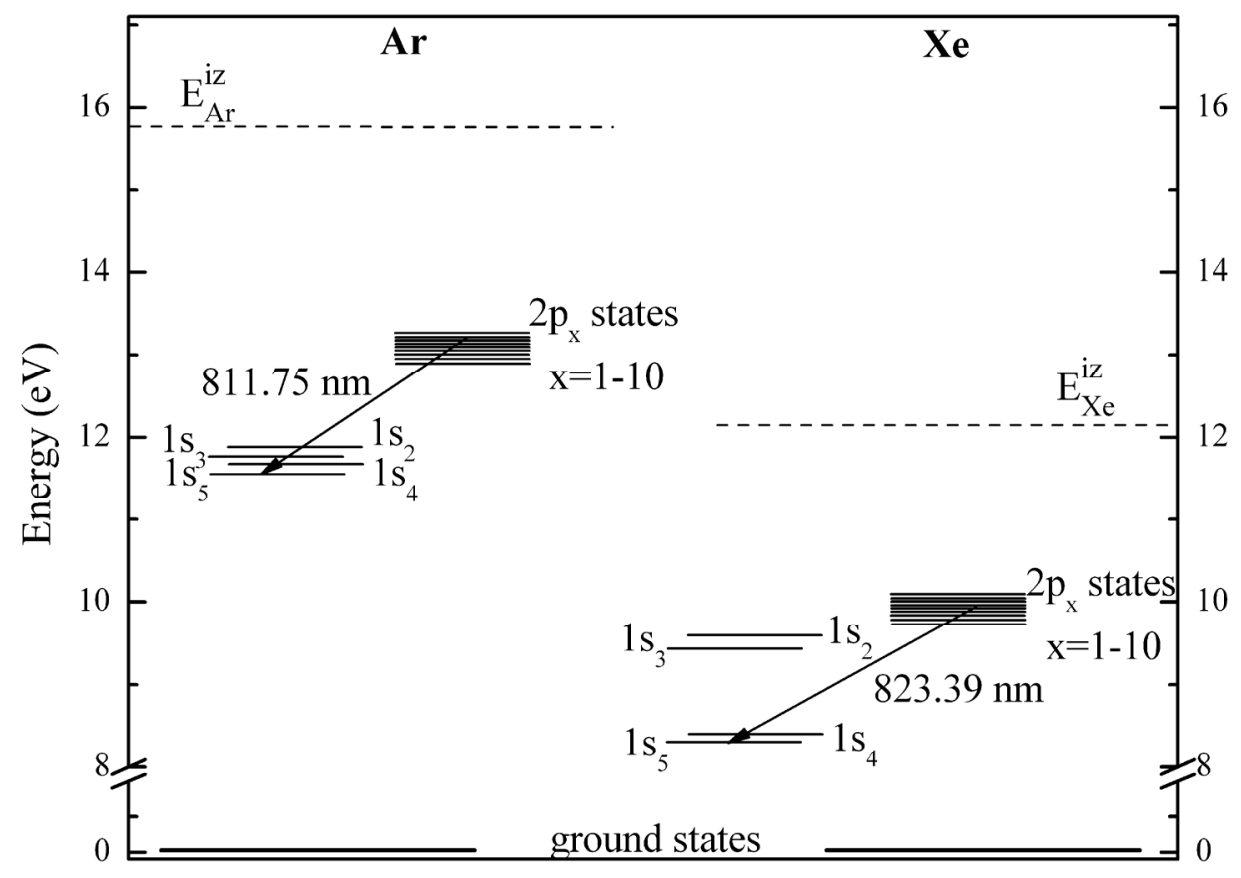

Figure 5.5. Partial energy level diagrams for argon and xenon neutrals and the transitions used for OES investigations; the ionization levels are shown by horizontal dashed lines; $1 \mathrm{~s}_{3}$ and $1 \mathrm{~s}_{5}$ are metastable states; $1 \mathrm{~s}_{2}, 1 \mathrm{~s}_{4}$, and $2 \mathrm{p}_{\mathrm{x}}$ are radiative states.

Independent of the details of the Corona model used, a major concern when performing any sort of OES analysis is the optical thickness of the plasma at a particular wavelength. As a rule of thumb in choosing the spectral lines for study, transitions to the ground level (resonance lines) or to low lying metastable levels should be avoided because the radiated photons are likely to be reabsorbed by the plasma. For our investigations we chose the $811.75 \mathrm{~nm}\left(4 \mathrm{p}^{2}[5 / 2]_{3} \rightarrow 4 \mathrm{~s}^{2}[3 / 2]_{2}\right.$ or in Paschen notation $2 \mathrm{p}_{9}$ $\left.\rightarrow 1 \mathrm{~s}_{5}\right)$ and $823.39 \mathrm{~nm}\left(6 \mathrm{p}^{2}[3 / 2]_{2} \rightarrow 6 \mathrm{~s}^{2}[3 / 2]_{2}\right.$ or in Paschen notation $\left.2 \mathrm{p}_{6} \rightarrow 1 \mathrm{~s}_{5}\right)$ lines for neutral argon and xenon, respectively. Both lines originate from $2 p$ manifolds (see Figure 5.5) and terminate on relatively high energy, $1 \mathrm{~s}_{5}$ metastable levels $(8.31 \mathrm{eV}$ for xenon and $11.54 \mathrm{eV}$ for argon above the ground level). Therefore, the plasma is optically thin at these wavelengths for our plasma conditions. The close proximity in wavelength 
of both lines also minimizes the range over which the optical sensitivity of the detection systems needs to be calibrated.

Emission intensities of the spectral lines of neutral argon at $811.75 \mathrm{~nm}$ and neutral xenon at $823.39 \mathrm{~nm}$ as a function of the xenon fraction, normalized to the measured emission intensities for the corresponding pure gas plasma, are shown in Figure 5.6 (a). The emitted light was collected radially at $z=126 \mathrm{~cm}$ (distance from the end of the HELIX source opposite the expansion chamber). The system parameters were magnetic field strengths of $700 \mathrm{G}$ and $10 \mathrm{G}$ in HELIX and LEIA, respectively and rf power of 750 $\mathrm{W}$ at a frequency of $9.5 \mathrm{MHz}$. The gas composition was varied by varying the individual argon and xenon mass flow rates for a constant total mass flow of $10 \mathrm{sccm}$. For pure argon, a mass flow of $10 \mathrm{sccm}$ corresponds to neutral pressures of 1.3 mTorr in HELIX and $0.14 \mathrm{mTorr}$ in LEIA. For pure xenon, the corresponding pressures were slightly larger; 1.5 mTorr in HELIX and 0.16 mTorr in LEIA.
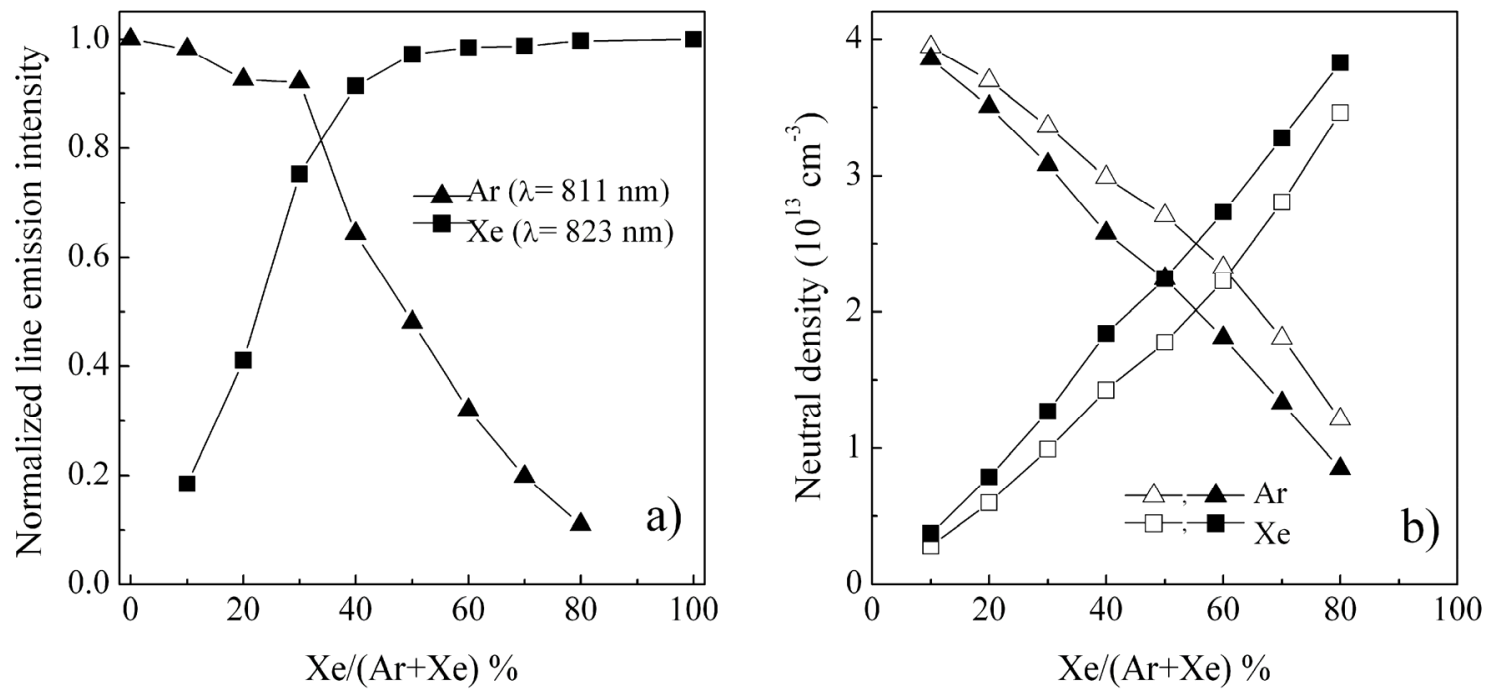

Figure 5.6. a) Observed emission line intensities from argon and xenon neutral lines in HELIX at $z=126$ $\mathrm{cm}$ versus xenon fraction; the emission intensities values are normalized to the corresponding pure gases values. The argon emission line intensity was indistinguishable from the background for xenon fractions larger than $80 \%$; b) Neutral species densities in the plasma as computed from the argon and xenon line intensities ratios: open symbols - Corona model without metastable contribution; full symbols - Corona model with metastable contribution.

As expected, the neutral argon emission intensity decreases and the neutral xenon emission intensity increases with increasing xenon fraction. For the general case that includes excitation from metastable states in the model, the ratio of the argon and xenon neutral line intensities given by Eq. (4.37) is 


$$
\frac{I_{811}}{I_{823}}=\frac{823}{811} \cdot \frac{S(811)}{S(823)} \cdot \frac{b_{811}}{b_{823}} \cdot \frac{k_{A r 0}^{811}}{k_{X e 0}^{823}} \cdot \frac{1+\frac{n_{A r}^{*} k_{A r *}^{811}}{n_{A r}^{0} k_{A r 0}^{811}}}{1+\frac{n_{X e}^{*} k_{X e^{*}}^{83}}{n_{X e}^{0} k_{X e 0}^{823}}} \cdot \frac{n_{A r}^{0}}{n_{X e}^{0}},
$$

where $n_{A r}^{0, *}$ and $n_{X e}^{0, *}$ are the neutral argon and xenon number densities in the ground " 0 " and metastable “*” states, respectively, and $k_{A r 0, *}^{811}$ and $k_{X e 0_{, *}^{*}}^{823}$ are the argon and xenon electron impact excitation rate coefficients from the ground and metastable states, respectively. The limit of only excitation from the ground states is obtained from Eq. (5.1) by setting the metastable densities $n_{A r}^{*}$ and $n_{X e}^{*}$ equal to zero.

The rate coefficients for electron impact excitation of neutral argon and xenon from the ground and from metastable states, as well as for excitation of argon and xenon ion state (the $434 \mathrm{~nm}$ and $529 \mathrm{~nm}$ emission lines for $\mathrm{Ar}^{+}$and $\mathrm{Xe}^{+}$that will be discussed later) are given in Table 5.1 as a function of electron temperature.

Table 5.1. Electron impact excitation rate coefficients for excitation of neutral argon and xenon from ground and metastable states $\left(k_{A r 0, *}^{811}, k_{X e 0, *}^{823}\right)$ and for $\mathrm{Ar}^{+}$and $\mathrm{Xe}^{+}$excitation from ground states $\left(k_{A r+}^{434}\right.$,

\begin{tabular}{|c|c|c|c|c|c|c|}
\hline $\begin{array}{c}T_{e} \\
(\mathrm{eV})\end{array}$ & $\begin{array}{c}k_{A r 0}^{811} \\
\left(10^{-12} \mathrm{~cm}^{3} \mathrm{~s}^{-1}\right)\end{array}$ & $\begin{array}{c}k_{A r^{*}}^{811} \\
\left(10^{-9} \mathrm{~cm}^{3} \mathrm{~s}^{-1}\right)\end{array}$ & $\begin{array}{c}k_{X e 0}^{823} \\
\left(10^{-12} \mathrm{~cm}^{3} \mathrm{~s}^{-1}\right)\end{array}$ & $\begin{array}{c}k_{X e^{*}}^{823} \\
\left(10^{-9} \mathrm{~cm}^{3} \mathrm{~s}^{-1}\right)\end{array}$ & $\begin{array}{c}k_{A r+}^{434} \\
\left(10^{-12} \mathrm{~cm}^{3} \mathrm{~s}^{-1}\right)\end{array}$ & $\begin{array}{c}k_{X e+}^{529} \\
\left(10^{-12} \mathrm{~cm}^{3} \mathrm{~s}^{-1}\right)\end{array}$ \\
\hline 3.8 & 2.35 & 3.21 & 8.38 & 1.39 & 1.29 & 1.82 \\
\hline 3.9 & 2.69 & 3.59 & 9.25 & 1.53 & 1.43 & 1.99 \\
\hline 4.1 & 3.39 & 4.32 & 11.34 & 1.87 & 1.73 & 2.39 \\
\hline 4.3 & 4.49 & 5.53 & 14.52 & 2.38 & 2.17 & 2.99 \\
\hline 4.5 & 8.20 & 8.81 & 24.43 & 3.98 & 3.52 & 4.82 \\
\hline 4.7 & 8.62 & 9.26 & 25.78 & 4.19 & 3.70 & 5.07 \\
\hline 5.0 & 13.90 & 13.78 & 39.37 & 6.38 & 5.51 & 7.51 \\
\hline 6.5 & 85.10 & 64.33 & 196.50 & 32.11 & 25.73 & 34.6 \\
\hline
\end{tabular}

They were calculated using Eqs. (4.34), the experimentally determined eedfs for different gas compositions, and the optical cross-sections available in the literature. ${ }^{3,4,5,6}$ For the neutral xenon transitions, the cross-section values were corrected for pressure effects 
resulting from radiation trapping of cascading resonance levels as described in Refs. $[6,7]$.

To calculate the absolute densities of the neutral species, Eq. (5.1) is combined with the gas kinetic equation

$$
p \cong\left(n_{A r}^{0}+n_{X e}^{0}\right) k_{B} T_{\text {gas }},
$$

where $p$ is the total neutral pressure, $k_{B}$ is the Boltzmann constant and $T_{g a s}$ is the gas temperature (assumed to be uniform throughout the plasma column and equal to the room temperature). The calculated neutral species densities are shown in Figure 5.6 (b). The branching ratios needed for these calculations (see Table 5.2) were calculated based on available transition probabilities and radiative level lifetimes. For the Corona model including metastable contributions to the line intensities, constant ratios of metastable population to the ground state population of $10^{-3}$ and $3 \times 10^{-3}$ for argon and xenon, respectively, were assumed. ${ }^{8}$

Table 5.2. Wavelengths, transition probabilities, upper level lifetimes, and branching ratios of the investigated neutral and ionic argon and xenon emission lines

\begin{tabular}{cccccc}
\hline \hline species & $\lambda(\mathrm{nm})$ & $A_{j i}\left(10^{7} \mathrm{~s}^{-1}\right)$ & $\tau(\mathrm{ns})$ & $b_{j i}$ & $\operatorname{Ref}(\mathrm{s})$. \\
\hline \hline $\mathrm{Ar}$ & 811.7 & 3.22 & 31 & 1.000 & 8 \\
$\mathrm{Xe}$ & 823.4 & 2.14 & 32.7 & 0.699 & 8 \\
$\mathrm{Ar}^{+}$ & 434.9 & 11.7 & 6.9 & 0.808 & 9 \\
$\mathrm{Xe}^{+}$ & 529.3 & 10.1 & 7.8 & 0.787 & 10,11 \\
\hline \hline
\end{tabular}

Since the measured light intensity is line-of-sight integrated, the calculated neutral densities correspond to radially uniform plasma, thereby excluding any profile effects that might occur on the axis of the helicon source. ${ }^{12}$ For the Corona model without metastable contributions, the calculated argon and xenon neutral densities show a departure from the expected linear dependence (open symbols in Figure 5.6 (b)) on mass flow rate composition. When metastable contributions are neglected, the calculated argon and xenon neutral densities are equal for a xenon fraction of $\sim 60 \%=\mathrm{Xe} /(\mathrm{Ar}+\mathrm{Xe})$. However, when the metastable excitation terms are included in the model, the calculated neutral densities exhibit a linear dependence on xenon fraction and the calculated densities are equal for a xenon fraction of $\sim 50 \%$ as expected. 
In a similar manner, the relative ion densities can also be determined through OES of ion emission lines. In this work, we examined the argon ion $4 \mathrm{p}{ }^{4} \mathrm{D}_{7 / 2} \rightarrow 4 \mathrm{~s}{ }^{4} \mathrm{P}_{5 / 2}$ transition with emission at $\lambda=434.93 \mathrm{~nm}$. For the xenon ion, we examined the $6 \mathrm{p}[2]_{5 / 2}$ $\rightarrow 6 \mathrm{~s}[2]_{5 / 2}$ transition with emission at $\lambda=529.37 \mathrm{~nm}$. In the process of populating excited ionic levels by electron impact, two mechanisms may contribute: excitation from the ion ground state and direct excitation from the atom ground state. Since the second mechanism implies simultaneous ionization and excitation, it requires highly energetic electrons. For instance, for excitation of the $4 p^{4} \mathrm{D}_{7 / 2}$ argon ion level from the ion ground state level, $19.5 \mathrm{eV}$ energy electrons are needed. Excitation from the argon atom ground state requires $15.8 \mathrm{eV}+19.5 \mathrm{eV}=35.3 \mathrm{eV}$. Since for our argon plasma the electron temperature is low $(\sim 7 \mathrm{eV})$, there are few electrons with the $35 \mathrm{eV}$ or larger energy in the tail of the distribution required for the simultaneous ionization and excitation of the atom in the ground state:

$$
\mathrm{Ar}_{0}+\mathrm{e}^{-}(\mathrm{E} \geq 35 \mathrm{eV}) \rightarrow \mathrm{Ar}^{+*} .
$$

Thus, the primary pathway for population of excited ionic levels is the two-step process, i.e., ionization of the neutral ground state and then excitation of the ion ground state:

$$
\begin{aligned}
& \operatorname{Ar}_{0}+\mathrm{e}^{-}(\mathrm{E} \geq 15.8 \mathrm{eV}) \rightarrow \mathrm{Ar}^{+} \text {and } \\
& \operatorname{Ar}^{+}+\mathrm{e}^{-}(\mathrm{E} \geq 19.5 \mathrm{eV}) \rightarrow \mathrm{Ar}^{+*} .
\end{aligned}
$$

A similar analysis is appropriate for the xenon. The $6 \mathrm{p}[2]_{5 / 2}$ excited $\mathrm{Xe}^{+}$level is populated by either the one-step process

$$
\mathrm{Xe}_{0}+\mathrm{e}^{-}(\mathrm{E} \geq 26 \mathrm{eV}) \rightarrow \mathrm{Xe}^{+*}
$$

which has a $26 \mathrm{eV}$ energy threshold, or by the two-step process

$$
\begin{aligned}
& \mathrm{Xe}_{0}+\mathrm{e}^{-}(\mathrm{E} \geq 12.2 \mathrm{eV}) \rightarrow \mathrm{Xe}^{+} \text {then } \\
& \mathrm{Xe}^{+}+\mathrm{e}^{-}(\mathrm{E} \geq 13.9 \mathrm{eV}) \rightarrow \mathrm{Xe}^{+*} .
\end{aligned}
$$

The latter process is considerably more likely given the low electron temperature $(\sim 4$ $\mathrm{eV}$ ) of the plasma. Three-step processes involving intermediate neutral or ion metastable states are also neglected.

Based on Eqs. (4.37) and (4.39), the relative ion to neutral emission line intensities for argon and xenon are given by 


$$
\frac{I_{434}}{I_{811}}=\frac{811}{434} \cdot \frac{S(434)}{S(811)} \cdot \frac{b_{434}}{b_{811}} \cdot \frac{k_{A r+}^{434}}{k_{A r 0}^{811}} \cdot \frac{1}{1+\frac{n_{A r}^{*}}{n_{A r}^{0} k_{A r}^{811}}} \cdot \frac{n_{A r}^{+}}{n_{A r}^{0}}
$$

and

$$
\frac{I_{529}}{I_{823}}=\frac{823}{529} \cdot \frac{S(529)}{S(823)} \cdot \frac{b_{529}}{b_{823}} \cdot \frac{k_{X e+}^{529}}{k_{X e 0}^{823}} \cdot \frac{1}{1+\frac{n_{X e}^{*} k_{X *}^{823}}{n_{X e}^{0} k_{X e 0}^{823}}} \cdot \frac{n_{X e}^{+}}{n_{X e}^{0}},
$$

where $n_{A r}^{+}$and $n_{X e}^{+}$are the ground state argon ion and xenon ion number densities, $k_{A r+}^{434}$ and $k_{X e+}^{529}$ are the $\mathrm{Ar}^{+}$and $\mathrm{Xe}^{+}$electron impact excitation rate coefficients from the ion ground state, and all the remaining quantities are the same meaning as in Eq. (5.1).

The ion/neutral emission line intensity ratios for argon and xenon are shown in Figure 5.7 (a) as a function of xenon fraction. Surprisingly, with increasing xenon fraction the ratio of argon ion line intensity to argon neutral line intensity increases, while the ratio of the xenon ion line to xenon neutral line intensity ratio decreases. These behaviors can be explained by the fact that the atomic emission lines reflect changes in the gas mixture composition while the ion line intensities are dominated by changes in the ionization processes.
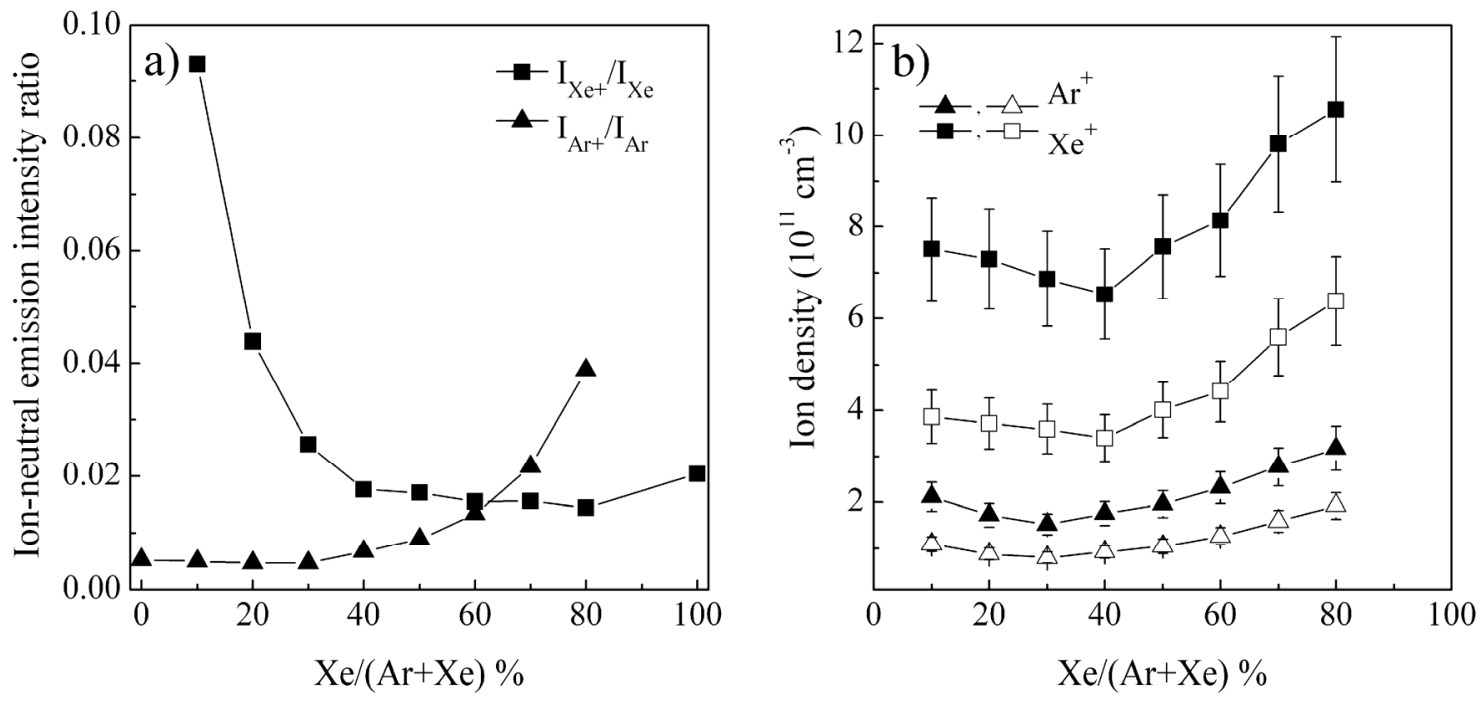

Figure 5.7. a) Ion to atomic emission line intensity ratio versus xenon fraction of the total mass flow rate; b) the inferred $\mathrm{Ar}^{+}$and $\mathrm{Xe}^{+}$densities based on a Corona model without metastable contribution (open symbols) and with metastable contributions (filled symbols) 
The calculated, from the measurements, $\mathrm{Ar}^{+}$and $\mathrm{Xe}^{+}$densities as a function of xenon fraction are shown in Figure 5.7 (b). To calculate the ion densities, Eq. (5.3) and the previously calculated neutral densities were used. Within experimental errors, the ion density of both species is roughly constant with increasing xenon fraction, up to a xenon fraction of $40 \%$. Above a xenon fraction of $40 \%$, both ion densities exhibit a linear increase with increasing xenon fraction. Inclusion of metastable contributions to the atomic line emission increases the calculated ion densities by a factor of 2 for both ion species (full symbols versus open symbols in Figure 5.7 (b)). Within experimental uncertainties estimated to be $\sim 15 \%$, the calculated $\mathrm{Xe}^{+}$and $\mathrm{Ar}^{+}$densities differ by a constant ratio of $\sim 3.5$ independent of the xenon fraction.

An increase in the $\mathrm{Xe}^{+}$density with increasing xenon mass flow fraction is expected. However, the increase in $\mathrm{Ar}^{+}$density with increasing xenon mass flow fraction from $40 \%$ to $80 \%$ is unexpected and highly improbable. To check the validity of the ion density calculation, the total ion density from the sum of the OES calculated ion densities and the electron densities measured by Langmuir probe are shown in Figure 5.8 as a function of xenon mass flow fraction. The electron density increases linearly with xenon fraction over the whole (10\% to $80 \%)$ xenon range. This result is consistent with the trends in the calculated ion densities versus xenon fraction for xenon fractions greater than $40 \%$. More significantly, the plasma quasineutrality condition,

$$
n_{A r}^{+}+n_{X e}^{+} \cong n_{e}
$$

is not satisfied by the OES and Langmuir probe measurements. The electron density is 9 to 11 times smaller than the total ion density calculated with the Corona model including metastable contributions and is 4 to 7 times smaller when the Corona model without metastable contribution is employed. With the caveats that the optical measurements were performed $\sim 50 \mathrm{~cm}$ downstream of the location where Langmuir probe measurements were obtained, that uncertainties associated with the optical emission cross-sections of the ionic lines are large $(\sim 35 \%$ for ionic lines compared to $\sim 10 \%$ for neutral lines) [3], and that many simplifying assumptions used in the emission line model, the values of the absolute ion densities provided by OES are reasonable. As noted by Boffard and co-workers, ${ }^{13}$ the "corona model is an extreme simplification of the plasma dynamics. It is well known that corona model fails for highly excited states which 
have decreased radiative transition rates and increased electron induced collisional mixing." Thus, given the uncertainties in the cross-section values and the probe measurements, the qualitative agreement between the OES and Langmuir probe measurements versus xenon fraction engenders confidence in the trends determined by analysis of the OES measurements.

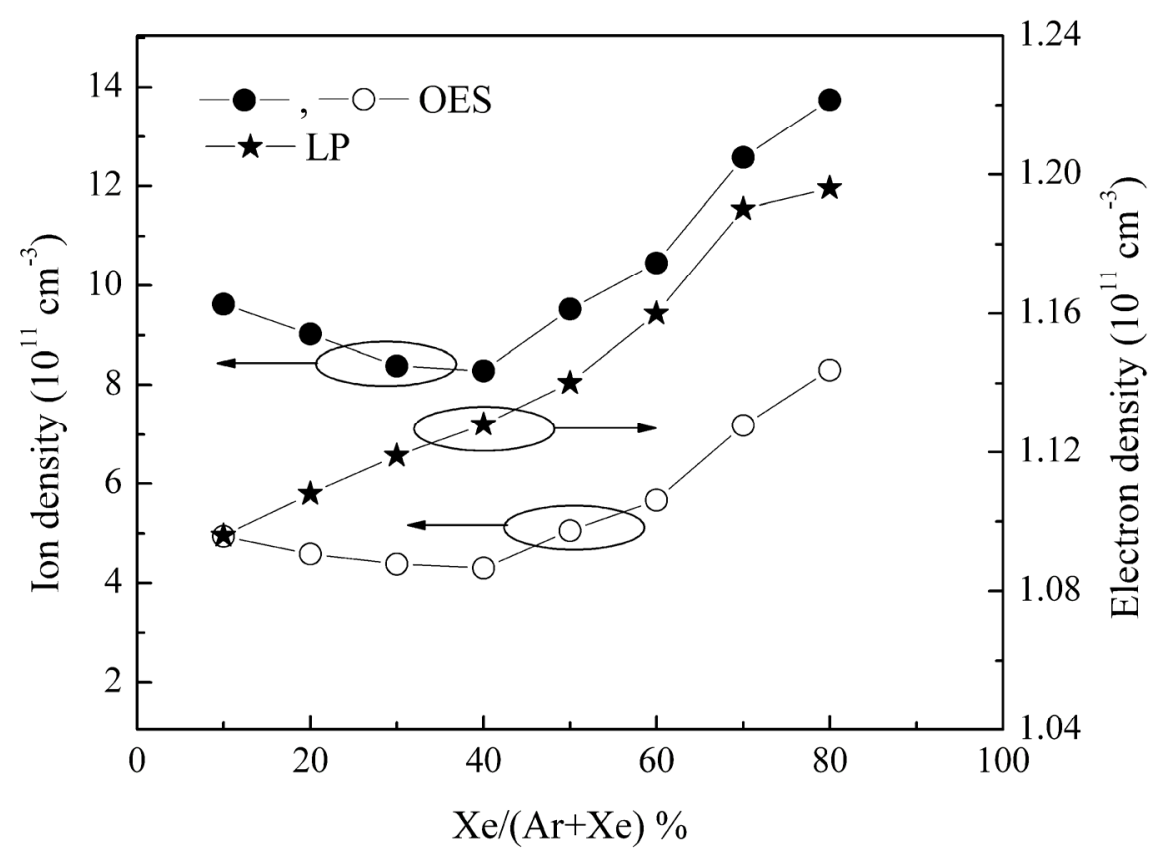

Figure 5.8. OES derived total ion densities based on a Corona model with no metastable contribution (open circles) and Corona model including metastable contribution (full circles), and Langmuir probe measured electron density (star symbols).

\subsection{Ion Velocity Distribution Function in Single Ion Species Plasma}

Previous studies have shown that in helicon discharges for operating gas pressures below a threshold value ( $\sim 2 \mathrm{mTorr}$ for Ar), a current free electric double layer (EDL) spontaneously appears near the source-diffusion chamber junction. ${ }^{14}$ For the HELIXLEIA helicon source-diffusion chamber system, the EDL forms at the axial location where the magnetic field gradient is the largest, i.e., $\sim 4 \mathrm{~cm}$ inside the source (see Figure 3.4). ${ }^{15}$ The signature of EDL formation is a downstream bimodal parallel ion velocity distribution function (ivdf) comprised of a slow and a fast ion population. These two ion populations supposedly have different origins: the slow ions are a background population created locally and the fast ions are created upstream in the source and accelerated by the 
EDL potential drop. Studies of EDL formation in other helicon sources have shown that the magnetic field profile and the magnetic field strength play an essential role in EDL formation and EDL strength. ${ }^{16,17}$ In the HELIX-LEIA system the magnetic field profile is fixed (fixed solenoids positions), but the strength of the magnetic fields in HELIX and LEIA can be varied independently. When the magnetic field strength was varied in the source, there was no effect on the speed of ions detected by laser induced fluorescence (LIF) downstream in the diffusion chamber. However, when the magnetic field in the source was kept constant and the magnetic field in the diffusion chamber decreased below a certain value of $\sim 70 \mathrm{G}$, the downstream ivdf changed from unimodal to bimodal. Further decrease of the LEIA magnetic field resulted in an increase in the speed of the fast ion component.

To explore and separate the effects of the magnetic field and electric double layer on ion acceleration, LIF measurements of the argon ion velocity distribution function were performed downstream of the EDL, in the divergent magnetic field region, at $z=$ $146 \mathrm{~cm}$ and $z=169 \mathrm{~cm}$, i.e., $-4 \mathrm{~cm}$ and $+19 \mathrm{~cm}$ from the HELIX-LEIA junction. To allow EDL formation, the argon pressure in HELIX was maintained at 1.5 mTorr. The corresponding LEIA pressure was 0.18 mTorr. The discharge was run at a constant input rf power of $800 \mathrm{~W}$ at a driving $\mathrm{rf}$ frequency of $9.5 \mathrm{MHz}$. The magnetic field in the source was held constant at $600 \mathrm{G}$.

Typical ivdfs at $z=146 \mathrm{~cm}$ and well downstream of the EDL at $z=169 \mathrm{~cm}$ are shown in Figure 5.9. The positive and negative frequency shifts of the fast ion distribution relative to iodine reference line, equivalently the positive and negative ion flow speeds shown at the top of the graphs in Figure 5.9 arise from the injection laser direction for each measurement. To obtain the parallel ivdf at first location, the laser was injected from the end of HELIX along the axis of the system (point $P_{2}$ in Figure 4.24) and the fluorescence light was collected radially. Since the bulk ion velocity is in the same direction as the laser propagation, i.e., from HELIX toward LEIA, the faster the ion velocity the larger the Doppler shift increase in the absorption frequency. 

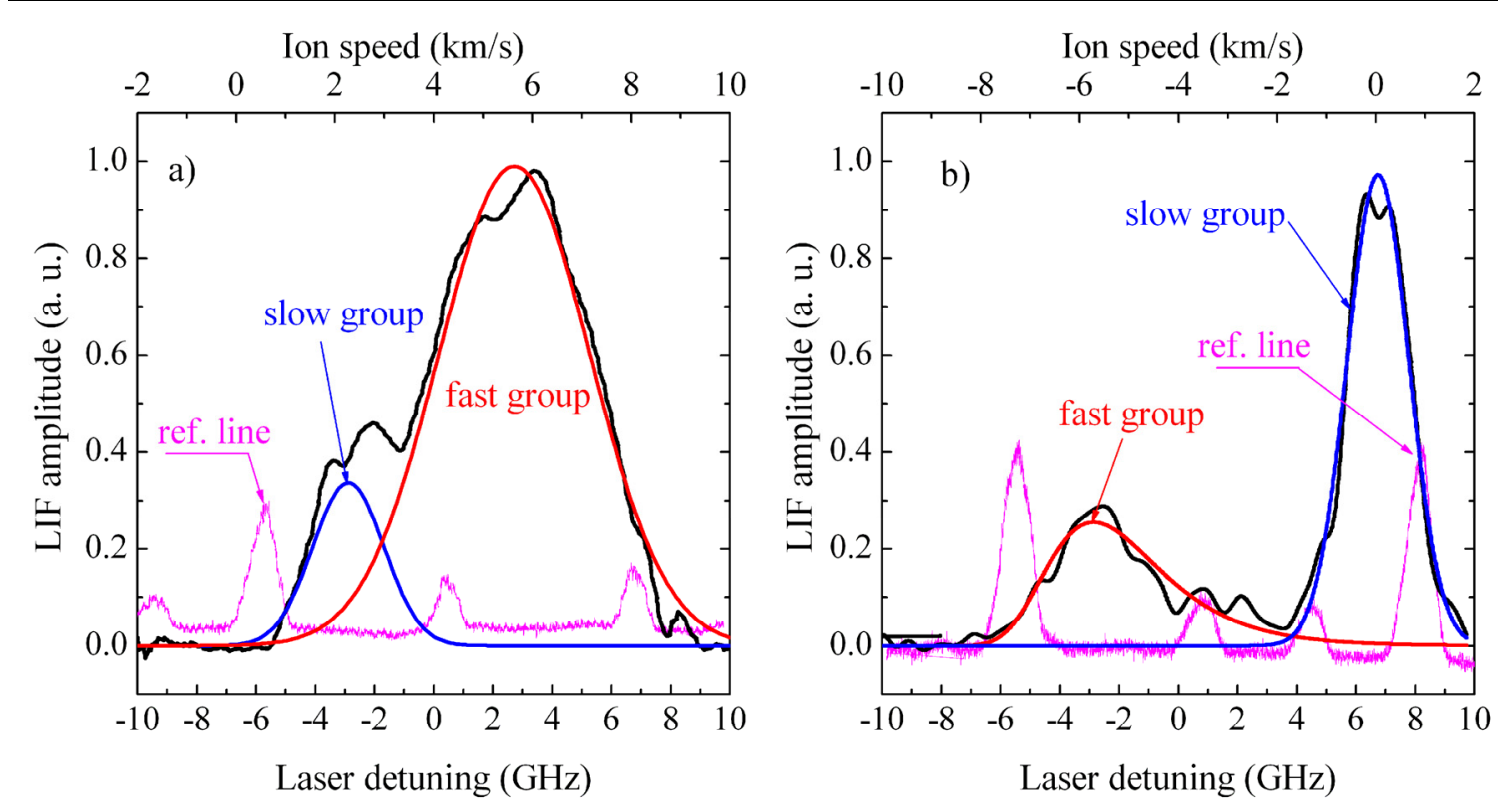

Figure 5.9. a) Argon ion parallel ivdf showing slow and fast ion populations in the source at $z=146 \mathrm{~cm}$; ) the ivdf in the expansion region at $z=169 \mathrm{~cm}$. Plasma parameters were: source gas pressure $1.5 \mathrm{mTorr}$, expansion chamber pressure $0.18 \mathrm{mTorr}$, input rf power $800 \mathrm{~W}$, rf frequency $9.5 \mathrm{MHz}$, source magnetic field $600 \mathrm{G}$ and expansion chamber magnetic field $35 \mathrm{G}$. In both graphs, the thick black lines are LIF signals; the red and blue curves are deconvolved fast and slow ion population ivdfs, respectively; the purple line is the iodine reference spectrum.

At the second location $(z=169 \mathrm{~cm})$, LIF measurements were performed with the scanning probe and the laser was injected towards the source from LEIA, to avoid blocking plasma flow. Therefore an ion moving towards LEIA will have its absorption frequency Doppler shifted to a lower frequency. Since the ivdfs at both locations have a bimodal structure, this is an indicative of an EDL upstream of $z=146 \mathrm{~cm}$. Note that it would be incorrect to conclude that there was no increase in the bulk ion speed from $z=$ $146 \mathrm{~cm}$ to $z=169 \mathrm{~cm}$. The speeds shown in Figure 5.10 (b) are based on raw data and must be corrected for the injection angle of the interrogating laser beam (see Figure 4.23 (b) ). As will be shown in the next chapter, the parallel flow speed value is roughly given by the LIF measured value divided by the cosine of the laser injection angle. Thus, a LIF determined fast group flow velocity of $-5.9 \mathrm{~km} / \mathrm{s}$ is equivalent to a downstream velocity of $+9.6 \mathrm{~km} / \mathrm{s}$ along the axis of the LEIA chamber. Another important observation is that the ratio of the fast to the slow ion population (proportional to the ratio of the integrated LIF intensities) at these two locations decreases substantially from its value of $\sim 6$ at $z=$ 
$146 \mathrm{~cm}$ to $\sim 0.5$ at $z=169 \mathrm{~cm}$. This dramatic decrease in fast population LIF signal is consistent with metastable state quenching over the $23 \mathrm{~cm}$ path. ${ }^{18,19}$

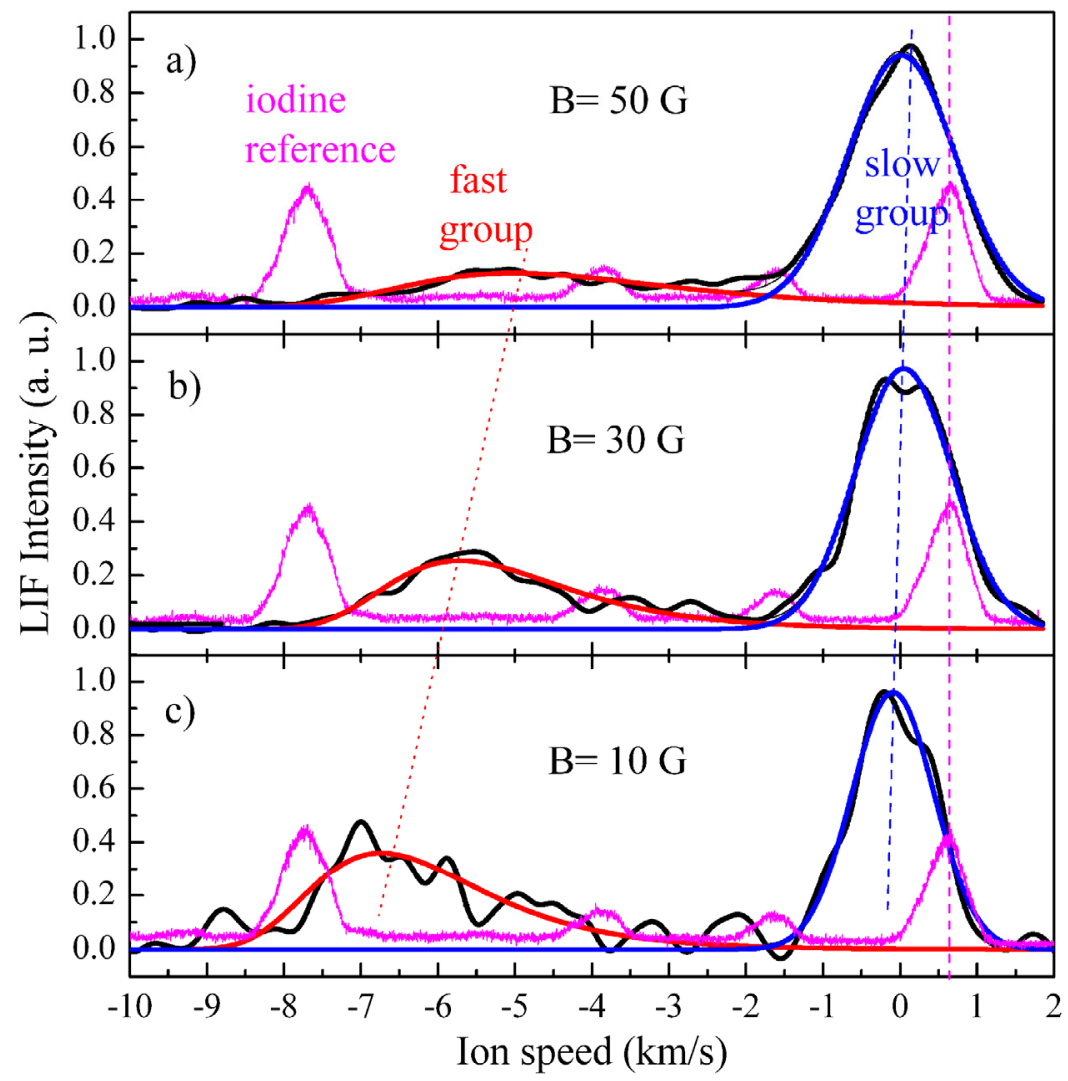

Figure 5.10. Bimodal argon ivdfs obtained in LEIA at $z=169 \mathrm{~cm}$ for a constant magnetic field strength in HELIX of $600 \mathrm{G}$ and magnetic fields in LEIA of: a) $\mathrm{B}=50 \mathrm{G}$; b) $\mathrm{B}=30 \mathrm{G}$; c) $\mathrm{B}=10 \mathrm{G}$. All other parameters are as in Figure 5.9. The distributions are normalized to the peak values of the slow ion populations. The raw LIF signals are shown by thick black lines; the fast and slow ion distributions by red and blue lines; the iodine reference spectrum by purple line; the centers of the slow ion distributions are indicated with dashed vertical line and the center of the fast ion distributions by an oblique dotted line.

Shown in Figure 5.10 are three parallel ivdf measurements corresponding to different magnetic field strengths in LEIA at $z=169 \mathrm{~cm}$. Similar to MNX observations, ${ }^{20}$ in which an increase in the speed of the fast ion component as a the strength of a nozzletype magnetic field was decreased was reported, a decrease of the magnetic field strength in LEIA has no effect on the flow velocity of the slow population but clearly increases the flow velocity of the fast population. Since the magnetic field strength in HELIX and all other parameters were held constant (and the ivdfs at $z=146 \mathrm{~cm}$ show little to no change as the magnetic field in LEIA is varied - so the EDL is effectively unchanged), the additional acceleration of the ions downstream of $z=146 \mathrm{~cm}$ must be due to the 
increase of the magnetic field divergence in the expansion region. After deconvolution and corrections for laser injection angle, the slow and fast ion populations parallel flow speeds versus LEIA magnetic field are shown in Figure 5.11. Except for two data points at very low magnetic field, the slow ions are practically at rest. The fast ion parallel speed increases sharply as the magnetic field decreases and saturates at $\sim 10.5 \mathrm{~km} / \mathrm{s}$ for LEIA magnetic fields below 30-40 G. At the lowest magnetic field strength of $7 \mathrm{G}$, the fast ion speed is supersonic $v_{z} \cong 2.9 c_{\mathrm{Ar}+}$.
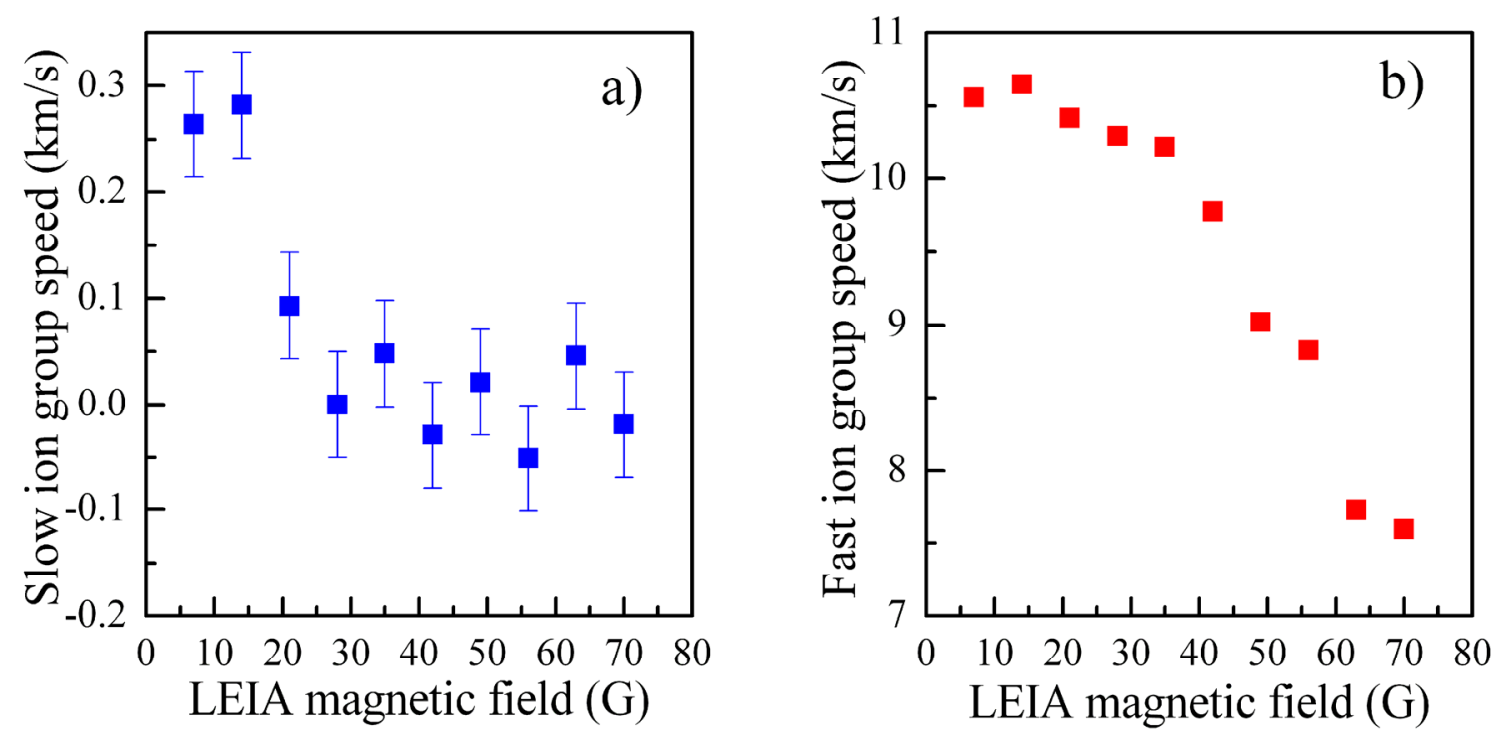

Figure 5.11. Corrected argon ion parallel flow speeds of the a) slow ion population and b) fast ion population as a function of LEIA magnetic field.

To better quantify the effect of the magnetic field divergence on the accelerated and background ion populations, the upstream/downstream magnetic field ratio, $R=$ $B_{H} / B_{L}$ (the ratio of the magnetic field strength in HELIX to the magnetic field strength in LEIA), is introduced. For these experiments the LEIA magnetic field ranged from 7-70 G, equivalent to a $R$ range of 9 to 86 . Note that at the measurement location (point $P_{1}$ in Figure 4.24), the ratio of the local magnetic field strength to the helicon source magnetic field strength only varies from 3.2 to 3.6. However, since the overall field geometry in the diverging region is determined by the $B_{H}$ and $B_{L}$ values in HELIX and LEIA, $B_{H}$ and $B_{L}$ are used in the definition of $R$.

Additional evidence in support of the conclusion that the slow ion population is created locally (in LEIA) and the fast ion population by passage through the EDL is 
provided by the slow and fast ion population LIF amplitudes. In LIF measurements, a qualitative predictor of the LIF signal amplitude (when the LIF signal is due to absorption out of metastable ion states created directly from the ion ground state) is the square of the electron density times the square root of the electron temperature. As expected and shown in Figure 5.12 (a), the slow ion population LIF intensity tracks the quantity $n_{e}^{2} T_{e}^{1 / 2}$ (with electron temperature and density determined from Langmuir probe measurements) whereas the LIF intensity of the fast group does not.
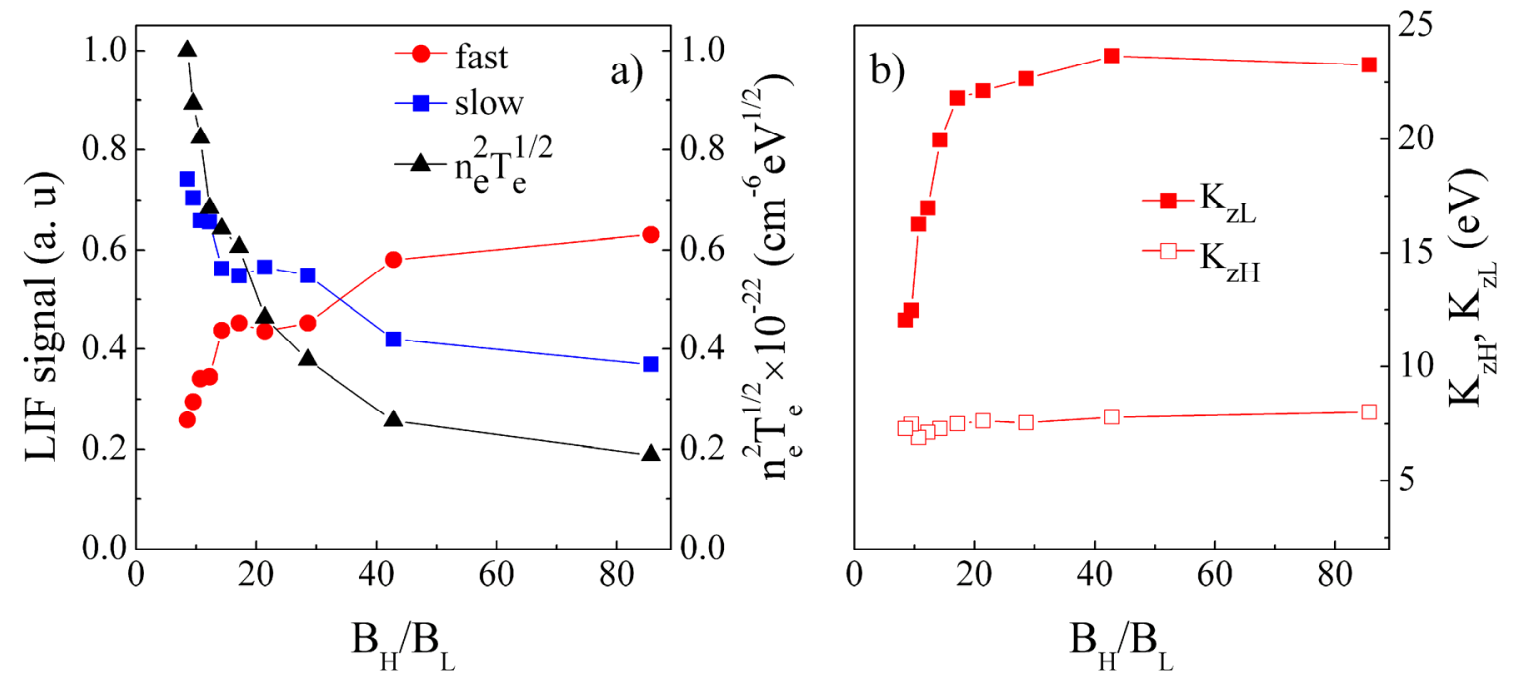

Figure 5.12. a) Dependence of the slow and fast ion populations LIF amplitudes and the quantity $n_{e}^{2} T_{e}^{1 / 2}$ on the HELIX-LEIA magnetic field ratio; b) Dependence of the parallel kinetic energy at the location $z=146$ $\mathrm{cm}$ (open symbols) and $z=169 \mathrm{~cm}$ (full symbols) versus HELIX-LEIA magnetic field ratio.

These results suggest that in addition to triggering the EDL formation [17], the divergent magnetic field provides additional ion acceleration. LIF measurements performed just upstream of the EDL $(z=146 \mathrm{~cm})$ indicate only a modest change in the fast ion axial flow speed and parallel kinetic energy with increasing HELIX/LEIA magnetic field ratio (Figure 5.12.b); a slight increase from $7.4 \mathrm{eV}$ to $8.1 \mathrm{eV}$ as the $B_{H} / B_{L}$ ratio increases from 9 to 86 . Thus, the substantially higher ion beam kinetic energy ( $\sim 15$ $\mathrm{eV}$ increase) observed in the expansion region, $19 \mathrm{~cm}$ downstream the HELIX-LEIA junction, cannot be due to an increase in the potential drop across the EDL. The location of the maximum magnetic field gradient also changes by only a few $\mathrm{mm}$ as the ratio is varied over the experimental range. As the ions travel from HELIX into LEIA, they 
experience the potential drop of the $\operatorname{EDL}\left(\phi_{D L}>0\right)$ and the mirror force $(-\mu \nabla \mathbf{B})$. Conservation of energy

$$
K_{H \|}+K_{H \perp}=e \phi_{D L}+K_{L \|}+K_{L \perp}
$$

and magnetic moment

$$
\frac{K_{H \perp}}{B_{H}}=\frac{K_{L \perp}}{B_{L}}
$$

gives

$$
K_{L \|}=K_{H \|}+e \phi_{D L}+K_{H \perp}(R-1) / R,
$$

where $K_{H \|}$ and $K_{H \perp}$ are the parallel and perpendicular ion kinetic energies in HELIX (upstream of the EDL) and $K_{L \|}$ is the parallel ion kinetic energy in LEIA (downstream of the EDL). Thus, only a fraction of the upstream perpendicular energy is converted into downstream parallel energy. Using the LIF measured velocity components, the perpendicular kinetic energy in HELIX needed to explain the 10-15 eV change in parallel kinetic energy in LEIA is $14-21 \mathrm{eV}$, far too large a quantity to be provided solely by the perpendicular ion temperature. One source of additional energy could be the conversion of azimuthal flow kinetic energy ${ }^{21}$ into parallel flow energy. The Lorentz force arising from azimuthal ion velocity and a radial magnetic field component in the diverging region is along the $z$ direction. However, previous measurements found only modest ( $0.8 \mathrm{eV}$ ) azimuthal flow energy. Another possible ion acceleration mechanism involves the balancing of upstream and downstream plasma pressure. Supersonic ion speeds $(\leq$ $3 c_{\mathrm{Ar}+}$ ) are predicted ${ }^{22}$ based on ion acceleration by the electron pressure gradient resulting from plasma expansion. Recent investigations of plasma expansion in the absence of a magnetic field demonstrated ion acceleration to supersonic speeds as the cross-sectional area expansion ratio was increased using different size plasma source chambers. ${ }^{23}$ In these experiments, the chamber diameters are fixed, but conservation of magnetic flux defines the plasma cross section in LEIA and the field expansion is physically equivalent to a change in plasma cross sectional area. The effective area expansion ratio is $A_{L} / A_{H}=B_{H} / B_{L}=R$. Thus, simple geometric expansion leading to both isentropic expansion and a decrease in electron pressure in the expansion chamber is the most likely explanation for the observed additional ion acceleration. 


\section{Chapter 5: Electron and Ion Energy Distribution Functions in Two-Ion Species, Helicon Plasmas}

\subsection{Ion Velocity Distribution Functions in Two-Ion Species Plasma}

Experiments have demonstrated that formation of a current free EDL in the divergent magnetic field at the end of helicon plasma source and subsequent ion acceleration to supersonic speeds occurs in a wide variety of working gases. Beside argon, ${ }^{24,25}$ ion acceleration in hydrogen ${ }^{26}$ and xenon $^{27}$ helicon plasma has been demonstrated. Ion beam velocities as high as $11 \mathrm{~km} / \mathrm{s}$ for $\mathrm{Ar}^{+}, 4.5 \mathrm{~km} / \mathrm{s}$ for $\mathrm{H}_{2}{ }^{+}$, and 6 $\mathrm{km} / \mathrm{s}$ for $\mathrm{Xe}^{+}$were measured with a retarding field energy analyzer (RFEA) in the ChiKung device. Also in Chi-Kung, very recent investigations in molecular gases found high ion exhaust speeds of $17 \mathrm{~km} / \mathrm{s}$ for $\mathrm{N}_{2}, 25 \mathrm{~km} / \mathrm{s}$ for $\mathrm{NH}_{3}$ and $27 \mathrm{~km} / \mathrm{s}$ for $\mathrm{CH}_{4}{ }^{28}$ Two distinct ion populations, one highly supersonic, have been also measured downstream of a small diameter compact helicon plasma source based on permanents magnets. ${ }^{29}$ In that experiment, the divergent magnetic configuration was found to be the crucial factor in ion beam formation: supersonic ion beams were observed only for a magnetic nozzle configuration. The magnetic nozzle requirement is consistent with the results presented in this work that suggest electrostatics is not the only driver for ion acceleration. Based on ion beam analysis in noble gas plasmas, Shamrai and co-workers [29] found that: a) the minimum gas pressure at which a stable discharge can be sustained and the most efficient ion acceleration occurs is lower in heavier gases (which for noble gases corresponds to lower ionization potentials); and b) for identical discharge conditions, the ion beam velocity decreases with atomic mass number and ranges from $58 \mathrm{~km} / \mathrm{s}$ in He to $24 \mathrm{~km} / \mathrm{s}$ in Ar to $13 \mathrm{~km} / \mathrm{s}$ in Xe. This second observation implies a dependence of ion acceleration on the characteristic Bohm speed (proportional to the inverse of the square root of the ion mass). However, it is difficult to conclusively demonstrate the role of the Bohm speed in defining the final ion speed in different single ion species plasma experiments because of the impossibility of matching the operating conditions (gas pressure, flow rate, input power etc) and electron temperature for different working gases. An alternative approach is to investigate ion acceleration in mixed gas plasmas with a single electron temperature. Under such conditions, ion acceleration controlled by the Bohm velocity will be easier to confirm. 


\section{Chapter 5: Electron and Ion Energy Distribution Functions in Two-Ion Species, Helicon Plasmas}

\subsubsection{Argon IVDF in Ar-Xe Mixture}

\subsection{1.a. Effect of Ar/Xe Ratio on the Argon IVDF}

As shown in section 5.3 and consistent with previous observations [25], an electric double layer (EDL) with the high potential side oriented toward HELIX and the low potential side oriented toward LEIA forms below a threshold pressure in the HELIXLEIA system for argon plasma. The EDL potential drop and the magnetic field expansion produce ion acceleration that results in a bimodal ivdf downstream of the EDL. Laser induced fluorescence (LIF) measurements of the parallel argon ion velocity distribution function revealed that EDL is located just upstream of the HELIX-LEIA junction, where the magnetic field gradient is a maximum (see Figure 3.4 (b)). In the following experiments, the parallel ivdf of argon and xenon ions is investigated as a function of gas mixing ratio to demonstrate EDL formation (if any) in Ar-Xe mixed plasma.

LIF measurements of the parallel ivdfs were performed on the axis of the source at $z=126 \mathrm{~cm}$ (location A in Figure 3.3). Discharges in pure Ar, Xe or their mixtures were obtained for constant external parameters: $700 \mathrm{G}$ magnetic field strength in HELIX, $10 \mathrm{G}$ magnetic field strength in LEIA and $750 \mathrm{~W}$ of input rf power. Since the rf energy coupling to the plasma varied from one gas composition to the other, matching network tuning for minimum reflected power was performed for each discharge condition. During operation, the reflected rf power was monitored in real time and maintained below $20 \mathrm{~W}$, i.e., less than $3 \%$ of the forward rf power. The variable parameter in these investigations was the gas composition, which was varied by modifying the individual argon and xenon mass flow rates while maintaining a constant $10 \mathrm{sccm}$ total mass flow rate. For this mass flow rate, the neutral pressure in the source was 1.3 mTorr for pure argon and slightly higher, $1.5 \mathrm{mTorr}$, for pure xenon. Because the plasma density decreases with increasing radial distance from the source axis, the ion plasma frequency term in the full lower hybrid frequency calculation becomes significant and the lower hybrid frequency at the plasma edge is smaller than on axis. ${ }^{30}$ As shown in previous HELIX experiments, optimal rf coupling is achieved when the rf frequency is close to the on-axis lower hybrid frequency. The calculated on-axis lower hybrid frequencies for argon and xenon are $\approx 7.5$ 
$\mathrm{MHz}$ and $\approx 4 \mathrm{MHz}$, respectively. In these experiments, the source was run at the slightly higher $\mathrm{rf}$ frequency of $9.5 \mathrm{MHz}$. For this operating $\mathrm{rf}$ frequency the plasma is quiescent and stable over prolonged periods and for a wide range of $\mathrm{Ar} / \mathrm{Xe}$ mixture ratios.

To accurately determine the bulk ion velocity, the ion density, and the ion temperature from the LIF measured ivdf, the LIF system must be calibrated in absolute laser frequency and signal amplitude. Although the resolution of the Burleigh 1800 wavemeter used in this work is sufficient to tune the dye laser to the appropriate wavelength, the wavemeter refresh rate is too slow for the laser scanning rates typically used for LIF measurements, $\sim 0.5 \mathrm{GHz} / \mathrm{s}$. Therefore, as described in Chapter 4 , an iodine absorption spectrum was recorded for each scan of the laser. To identify the appropriate iodine lines to be used as a zero velocity reference for the LIF measurements, the Salami reference iodine spectrum ${ }^{31}$ was compared to experimentally obtained iodine spectra in the range of interest for each LIF scheme. As can be seen in Figure 5.13, for the $\mathrm{Ar}^{+}$ $611.6616 \mathrm{~nm}\left(16348.91 \mathrm{~cm}^{-1}\right)$ absorption line, the closest iodine line with a sufficient intensity is the $16348.94 \mathrm{~cm}^{-1}$ line. The absolute velocity corresponding to the $\mathrm{Ar}^{+}$line is given by

$$
V \cong \lambda_{0} \Delta v_{\text {total }},
$$

where the velocity $V$ is in $\mathrm{m} / \mathrm{s}$ if the rest frame wavelength $\lambda_{0}$ is in $\mathrm{nm}$ and the frequency shift $\Delta v_{\text {total }}$ is in GHz. For zero absolute velocity in the lab frame, the frequency difference between the iodine line and the $\mathrm{Ar}^{+}$absorption line is $1.08 \mathrm{GHz}$. 


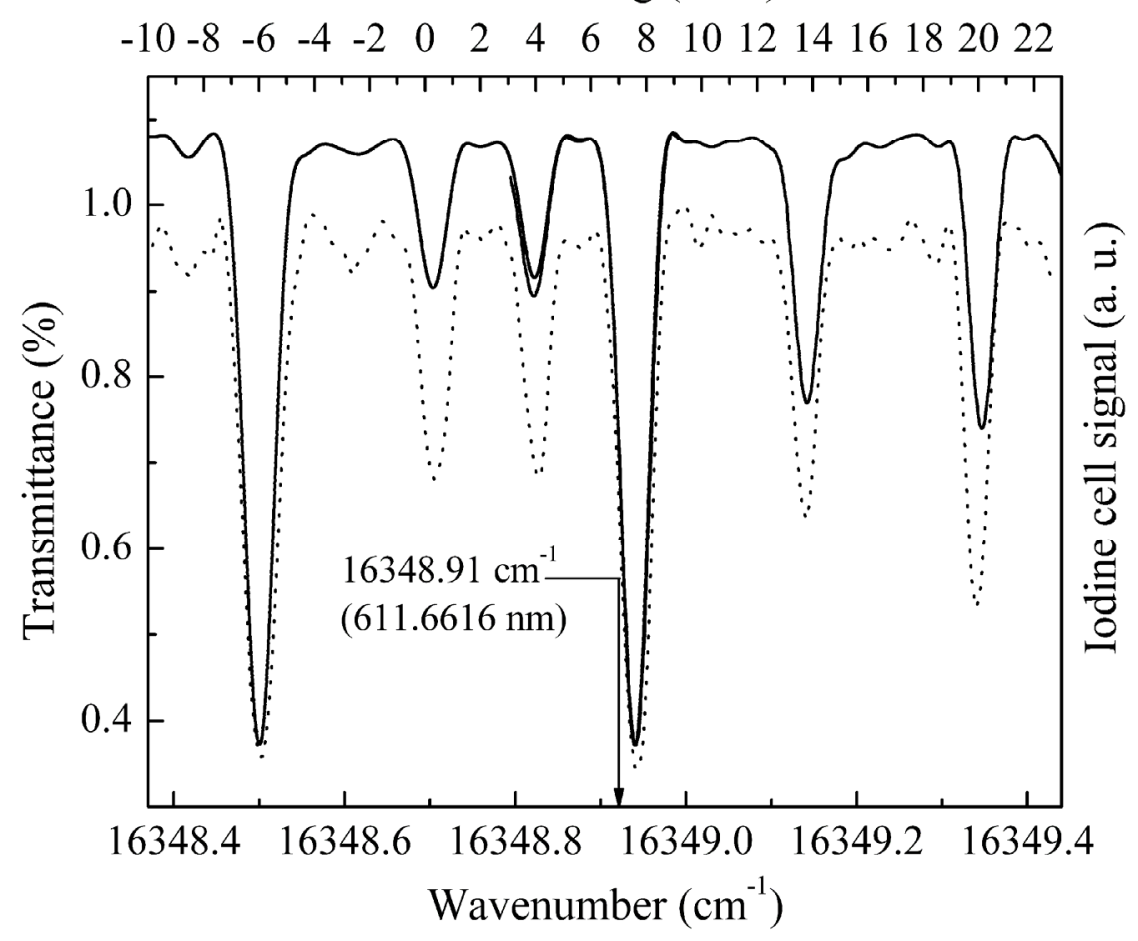

Figure 5.13. The iodine spectrum obtained from two partially overlapping $20 \mathrm{GHz}$ width laser scans (solid line) and the iodine spectrum according to Ref. [31] (dotted line) in the spectral range of interest for $\mathrm{Ar}^{+}$ LIF. The arrow indicates the rest frame value of $611.6616 \mathrm{~nm}$ (vacuum wavelength) $\mathrm{Ar}^{+}$line.

A similar identification and analysis of iodine lines was performed for the $\mathrm{Xe}^{+}$ LIF scheme. The most intense iodine line in the relevant wavelength range is at 16521.45 $\mathrm{cm}^{-1}$ (see Figure 5.14). With an accurate value for the rest frame $\mathrm{Xe}^{+}$transition wavelength, the absolute shift in the LIF measured absorption line can also be determined. Available tables ${ }^{32,33}$ based on early measurements by Humphrey ${ }^{34}$ provide a value for the $\mathrm{Xe}^{+}$wave number of $16521.22 \mathrm{~cm}^{-1}$. Based on interferometric measurements, in a later paper, ${ }^{35}$ Humphrey reported an improved value of 16521.285 $\mathrm{cm}^{-1}$. Hansen and Persson ${ }^{36}$ also reported a wave number of $16521.22 \mathrm{~cm}^{-1}$. The most recent wave number values are by Sadeghi, ${ }^{37}$ who reported a wave number value of $16521.299 \mathrm{~cm}^{-1}$ based on Lamb dip spectroscopy and by Cedolin et al. ${ }^{38,39}$ who reported a value of $16521.23 \mathrm{~cm}^{-1}$ based on direct and reflected laser LIF. Converted into frequency, the variation between maximum and minimum reported wave number values $\left(\sim 0.08 \mathrm{~cm}^{-}\right.$ $\left.{ }^{1}\right)$ spans $2.4 \mathrm{GHz}$. In terms of ion speed, this uncertainty corresponds to a speed uncertainty of $\sim 1.5 \mathrm{~km} / \mathrm{s}$. Therefore, measurements of perpendicular $\mathrm{Xe}^{+}$ivdf on the 
LEIA axis were used to obtain an absolute zero velocity reference wave number of $16521.332 \mathrm{~cm}^{-1}$. The frequency difference between the reference iodine line and the rest frame $\mathrm{Xe}^{+} 605.2781 \mathrm{~nm}$ absorption line is then $3.91 \mathrm{GHz}$.

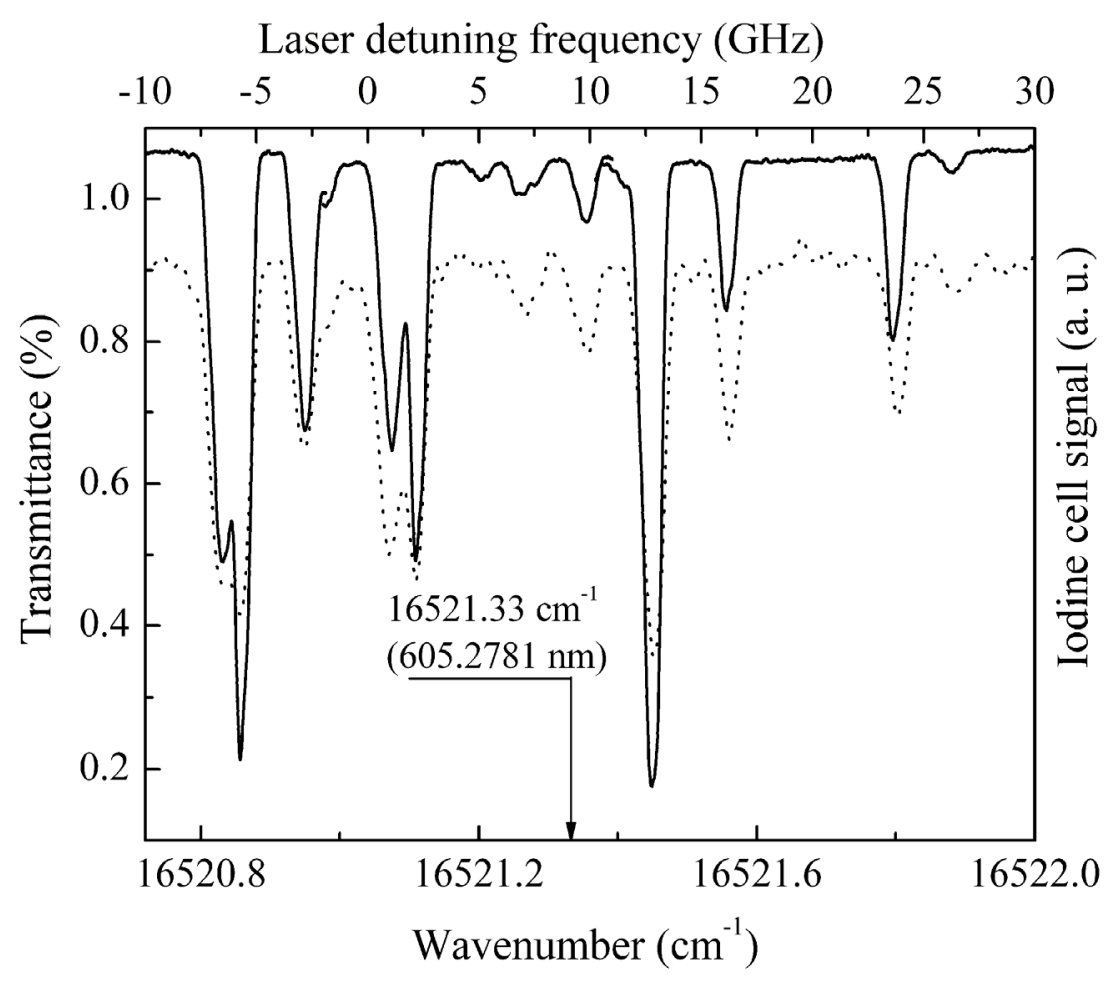

Figure 5.14. The iodine spectrum obtained from three $15 \mathrm{GHz}$ partially overlapping laser scans (solid line) and the iodine spectrum according to Ref. [31] (dotted line) in the spectral range of interest for Xe $\mathrm{e}^{+}$LIF. The arrow indicates the rest frame position of $605.2781 \mathrm{~nm}$ (vacuum wavelength) $\mathrm{Xe}^{+}$line.

The parallel ivdf of $\mathrm{Ar}^{+}$in HELIX is shown in Figure 5.15. The bulk ion velocity is calculated according to Eq. (5.6) for the total frequency difference between the LIF signal peak and the iodine reference line $(1.46 \mathrm{GHz}$ in this case) plus the $1.08 \mathrm{GHz}$ between the iodine line and the rest frame $611.66 \mathrm{~nm}$ line position minus $1.03 \mathrm{GHz}$ for the Zeeman shift at this magnetic field strength (this is a $\sigma^{+}$Zeeman line). The $\mathrm{Ar}^{+}$ parallel ivdf is well fit with a single Gaussian function. From the full width at half maximum (Eq. 4.41), the parallel argon ion temperature is $0.16 \mathrm{eV}$. 


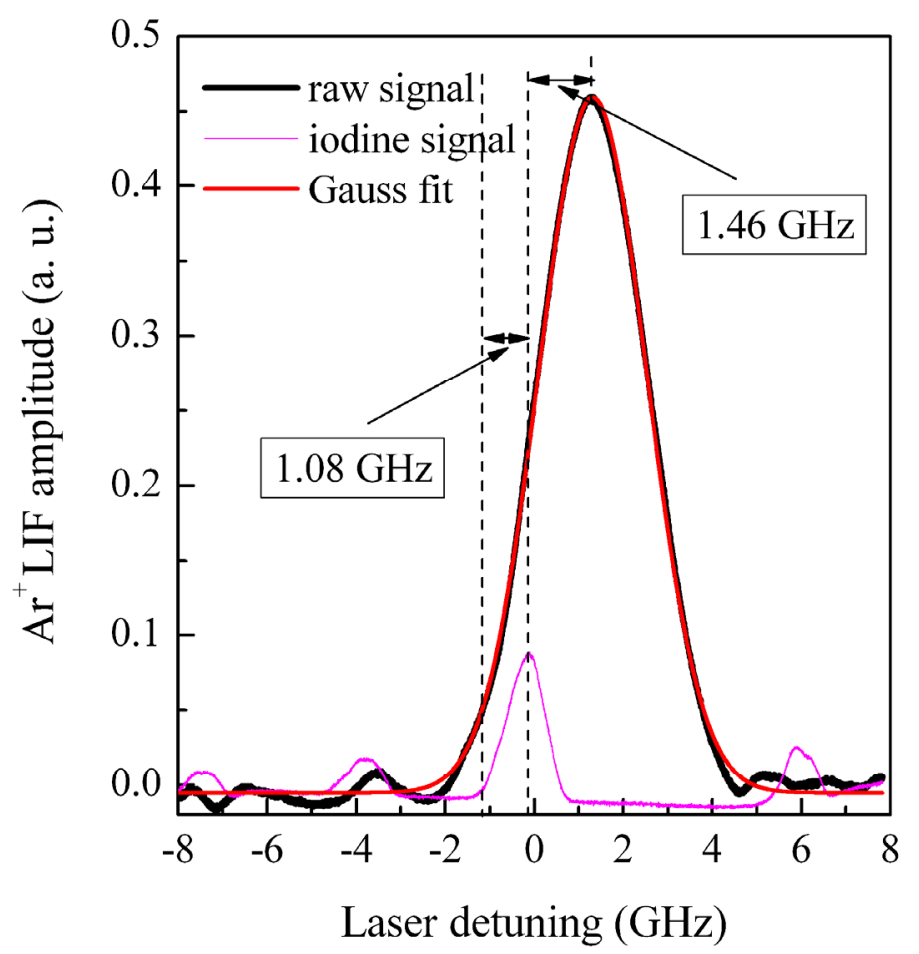

Figure 5.15. LIF profile of the $\operatorname{Ar}^{+} 611.66 \mathrm{~nm}$ line in HELIX at $z=126 \mathrm{~cm}$; black line is the raw LIF signal, the red line is a Gaussian fit, and the purple line is the iodine reference spectrum.

Due to the isotopic composition of natural xenon and the presence of a non-zero nuclear spin in odd isotopes 129 and 131 ( $\mathrm{I}=1 / 2$ and 3/2 respectively), the absorption spectrum of $\mathrm{Xe}^{+}$spreads over approximately $8 \mathrm{GHz}$ and interpretation is much more difficult than for argon (see Figure 5.16). In calculating the peak position (associated with ${ }^{132} \mathrm{Xe}$ isotope due to its highest abundance of $27 \%$ ) relative to the rest frame frequency, the zero velocity frequency difference of $3.91 \mathrm{GHz}$ between the $\mathrm{Xe}^{+}$line and the iodine line must be subtracted from the measured frequency difference of $2.28 \mathrm{GHz}$. The Zeeman shift of $1.26 \mathrm{GHz}(1.8 \mathrm{GHz} / \mathrm{kGauss} \times 0.7 \mathrm{kGauss})$ is then subtracted to obtain the total frequency shift of the $\mathrm{Xe}^{+}$absorption line. The LIF measured $\mathrm{Xe}^{+}$line shape is clearly non-Gaussian. As discussed in the previous chapter, the xenon hyperfine splitting can be modeled as a sum of nineteen individual hyperfine lines. Therefore, the $\mathrm{Xe}^{+}$ivdf must be deconvolved with nineteen Gaussian profiles (blue lines in Figure 5.16), centered at each of the nineteen component hyperfine line centers. As shown in Figure 5.16, the sum of the nineteen Gaussian profiles is in excellent agreement with the LIF 
measurement. It is worth noting that in contrast to the argon measurements, the parallel $\mathrm{Xe}^{+}$ion temperature cannot be uniquely determined.

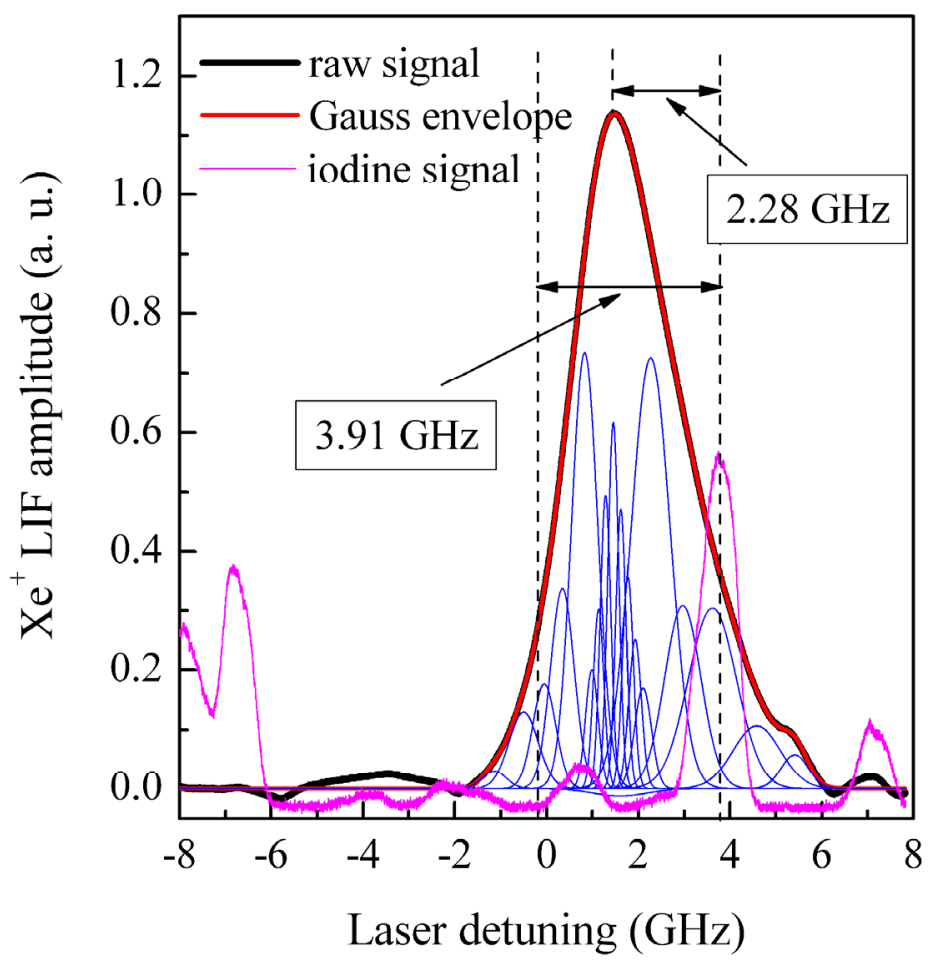

Figure 5.16. LIF profile of the $\mathrm{Xe}^{+} 605.28 \mathrm{~nm}$ line in HELIX at $z=126 \mathrm{~cm}$; black line is the raw LIF signal, the blue lines are Gaussian fits to the 19 components, the red line is the envelope of the fits, and the purple line is the iodine reference spectrum.

The relationship between the total LIF intensity and ion density is complex. Therefore, for absolute measurement of the ion density from a LIF measurement a complete calibration with a second method, such as Rayleigh scattering, is necessary. ${ }^{40}$ Under the assumption that the main population channel of the metastable ion level interrogated via LIF is electron impact excitation from the ion ground state,

$$
n_{m} \sum_{p<m} A_{p m} \cong n_{e} n_{i}\langle\sigma v\rangle_{0 m},
$$

where $n_{m}$ and $n_{i}$ are the metastable density (proportional to the LIF signal) and the ion ground state density, respectively, and $n_{e}$ is the electron density; $\langle\sigma v\rangle_{0 m}$ is the rate coefficient for electron impact excitation from the ion ground state to the $m$ excited state, and $\sum_{p<m} A_{p m}$ is the sum of the spontaneous transition probabilities from the metastable 
state to all lower states, the LIF signal has been found to be roughly proportional to a composite parameter given by the square of the electron density times the square root of the electron temperature ${ }^{41}$

$$
I_{L I F} \sim n_{e} n_{i} T_{e}^{1 / 2} \cong n_{e}^{2} T_{e}^{1 / 2} .
$$

This proportionality also assumes that cascading effects from upper states do not contribute significantly to the interrogated metastable state population. Although it is not an absolute calibration, Eq. (5.8) provides a qualitative correlation between the LIF signal (the metastable ion population) and the ground state ion population (assumed to be equal to the electron density). The validity of this proportionality was demonstrated experimentally for $\mathrm{Ar}^{+}$LIF in pure Ar plasma by Sun et al. ${ }^{42}$

To determine if Eq. (5.8) holds for a mixed argon and xenon plasma, each ion density was calculated based on the two-ion species fluid model described in Chapter 4. For electron temperatures and electron densities from Langmuir probe measurements and the computed ionization rate coefficients (Eq. 4.6), the calculated $\mathrm{Xe}^{+}$and $\mathrm{Ar}^{+}$absolute densities versus xenon fraction are shown in Figure 5.17.

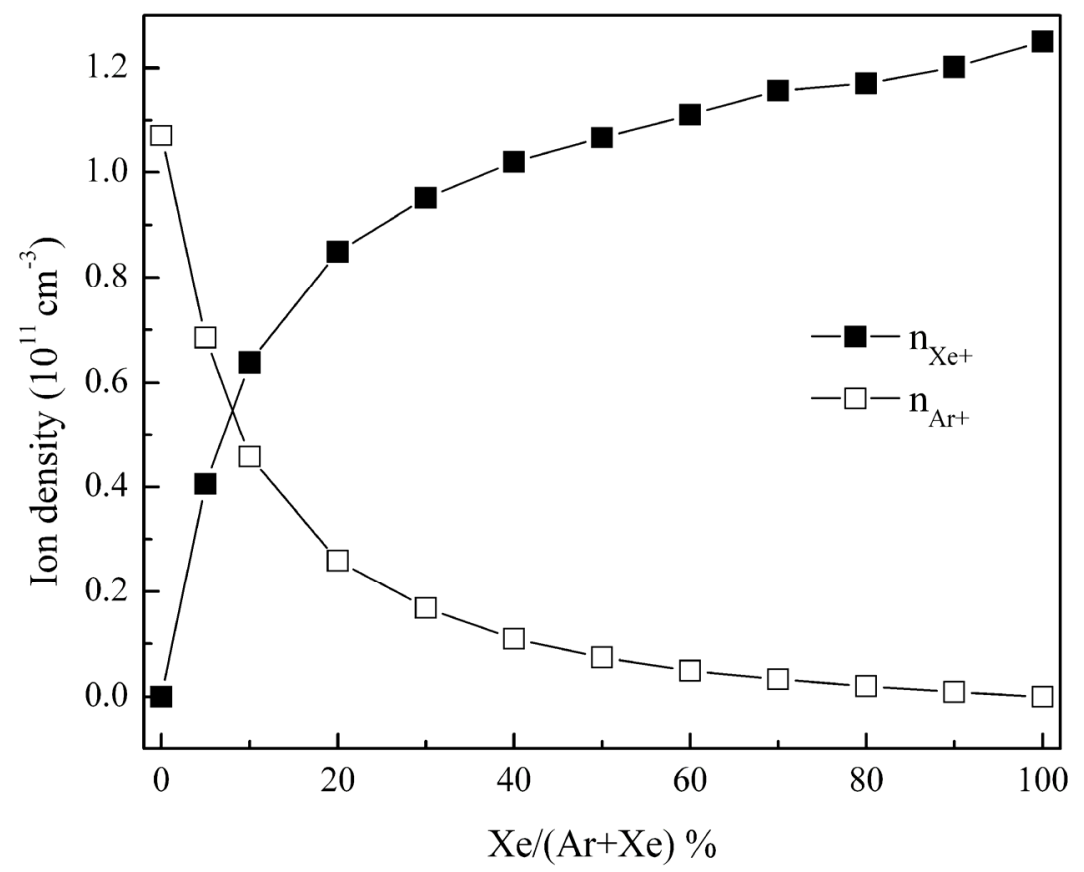

Figure 5.17. Computed ion densities in HELIX at $z=126 \mathrm{~cm}$ as a function of xenon fraction. 
Instead of the linear dependence on xenon fraction found for neutral species, an exponential decrease for $\mathrm{Ar}^{+}$density and a logarithmic increase for the $\mathrm{Xe}^{+}$density were obtained. Roughly equal ion densities of $5.5 \times 10^{10} \mathrm{~cm}^{-3}$ are obtained for xenon fraction of only $\sim 10 \%$. This extreme sensitivity to the xenon fraction results from the $3.7 \mathrm{eV}$ difference in ionization potential between argon and xenon and the twofold larger ionization cross-section for xenon. The combination of these differences causes the electron temperature to decreases rapidly with the xenon ratio (see Figure 5.1(a)). In other words, addition of xenon effectively "clamps" the electron energy distribution function and impedes ionization of argon. Similar dependencies of $\mathrm{Ar}^{+}$and $\mathrm{Xe}^{+}$densities versus xenon fraction were observed in capacitively coupled plasmas. ${ }^{43}$ In those experiments, the xenon fraction at which equal argon and xenon ion densities were measured was $\sim 15 \%$ (Fig. 5 in Ref. [43]), in excellent agreement with the value calculated here.

The measured $\mathrm{Ar}^{+}$and $\mathrm{Xe}^{+}$LIF intensities are compared with the product of electron density, computed ion density, and square root of the measured electron temperature in Figure 5.18. The measured argon LIF signal, and by implication the $\mathrm{Ar}^{+}$ metastable population, decreases with increasing xenon fraction until the signal disappears completely for xenon concentration greater than $25 \%$. This is consistent with the calculated $76 \%$ decrease in the $\mathrm{Ar}^{+}$density and $2 \mathrm{eV}$ decrease in electron temperature. In other words, the $\mathrm{Ar}^{+}$ground state population is too small and there are not enough high energy electrons to populate the $3 \mathrm{~d}^{2} \mathrm{G}_{9 / 2}$ ion metastable level located $19.11 \mathrm{eV}$ above the argon ion ground level. Conversely, the $\mathrm{Xe}^{+}$LIF signal shows a gradual increase with xenon fraction. The jumps in $\mathrm{Xe}^{+} \mathrm{LIF}$ signal at $50 \%$ and $90 \%$ xenon fraction are due to the changes in rf matching conditions. With the caveats about the assumptions used in the model and being aware that the Langmuir probe data were obtained on different run days than the LIF data, the qualitative agreement between the LIF signals and $n_{e} n_{i} T_{e}^{1 / 2}$ suggests that the LIF intensity does provide a rough indication of the species ion density (times the electron density and the square root of the electron temperature) in a mixed argon-xenon plasma. 

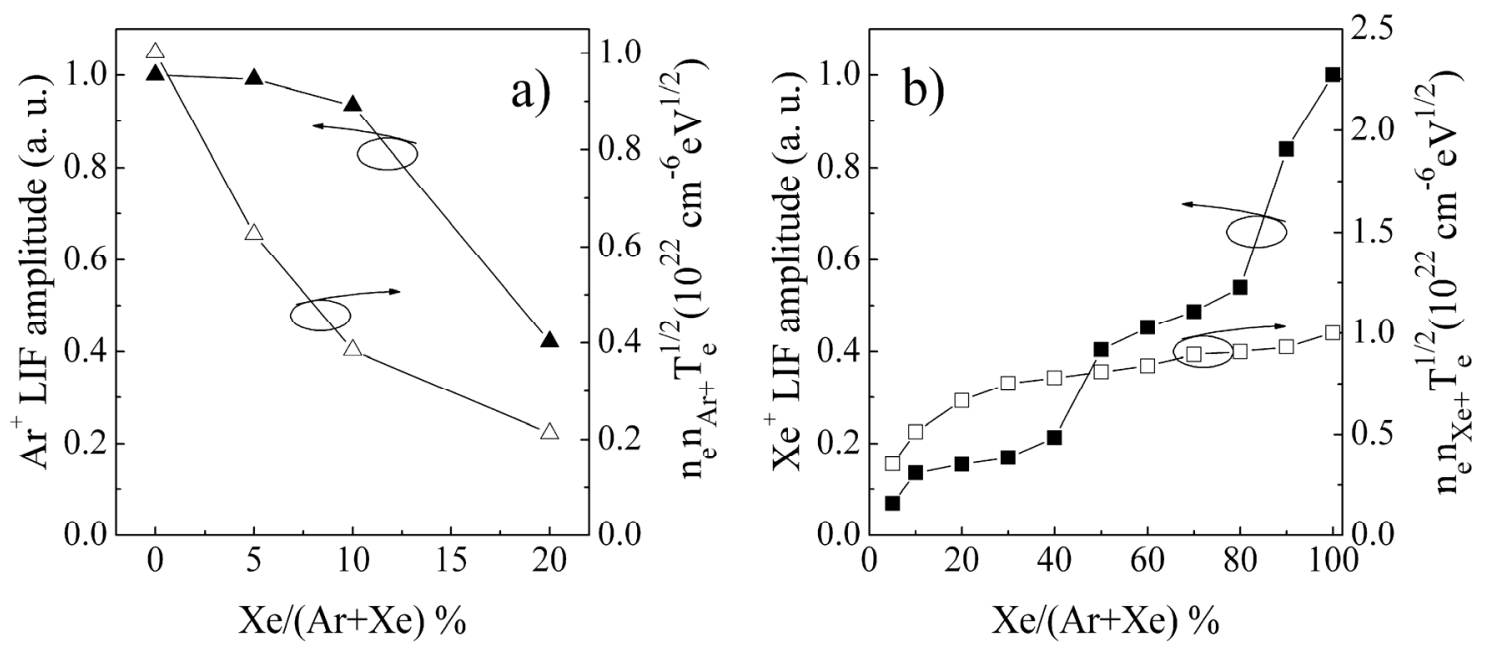

Figure 5.18. a) $\mathrm{Ar}^{+}$LIF intensity (solid triangles) and $n_{e} n_{A r+} T_{e}^{1 / 2}$ product (open triangles) and b) $\mathrm{Xe}^{+} \mathrm{LIF}$ intensity (solid squares) and $n_{e} n_{X e} T_{e}^{1 / 2}$ product (open squares) versus xenon fraction in HELIX at $z=126$ $\mathrm{cm}$. Plasma parameters were: $\mathrm{P}_{\mathrm{rf}}=750 \mathrm{~W}, f=9.5 \mathrm{MHz}, B_{H}=700 \mathrm{G}, B_{L}=10 \mathrm{G}$, and $F=10 \mathrm{sccm}$.

As discussed in Chapter 2, ion species enter an EDL either with their individual Bohm speeds $\left(c_{A r+, X e+}=\sqrt{\gamma k_{B} T_{e} / M_{A r, X e}}\right.$, where $\gamma=1$ for isothermal expansion) or the system Bohm speed

$$
c_{\text {system }}=\left(\frac{n_{X e+}}{n_{e}} c_{X e+}^{2}+\frac{n_{A r+}}{n_{e}} c_{A r+}^{2}\right)^{1 / 2} .
$$

Since the electron temperature varies with xenon fraction (see Figure 5.1(a)), the individual and system ion sound speeds also vary. Figure 5.19 shows the computed Bohm speeds at $z=126 \mathrm{~cm}$ in HELIX, upstream of the EDL, based on Langmuir probe measurements. The argon and xenon individual ion sound speeds are largest in the pure argon plasma and decrease slightly with increasing xenon fraction. The system ion sound speed follows the trend in electron temperature and decreases sharply with the xenon fraction; from the argon ion sound speed $\left(c_{\mathrm{Ar}^{+}}=4000 \mathrm{~m} / \mathrm{s}\right)$ in pure argon plasma to the xenon ion sound speed $\left(c_{\mathrm{Xe}+}=1700 \mathrm{~m} / \mathrm{s}\right)$ in pure xenon plasma. The largest change in system ion sound speed occurs as the xenon fraction changes from 0 to $25 \%$. 


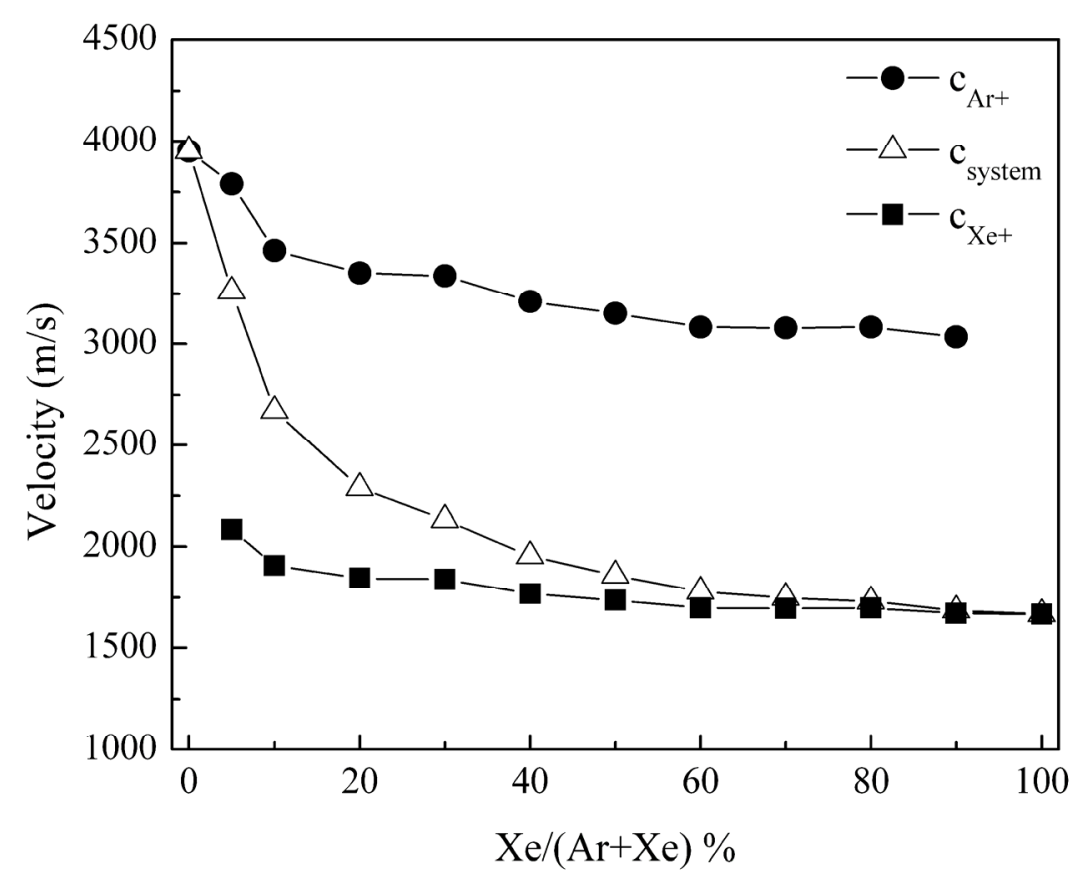

Figure 5.19. Individual ion sound speeds and the system sound speed as function of xenon fraction in HELIX at $z=126 \mathrm{~cm}$.

Experimentally determined parallel ion flow velocities for argon and xenon ions at the same spatial location in HELIX $(z=126 \mathrm{~cm})$ versus xenon fraction are shown in Figure 5.20. The measured speeds are much lower than the individual ion sound speeds and are independent of xenon fraction. Since the ratio of the argon to xenon speed is approximately constant at 1.7 and consistent with $\sqrt{M_{X e} / M_{A r}} \cong 1.8$, these measurements suggest the presence of a slight axial potential gradient that accelerates argon and xenon ions to speeds in a manner inversely proportional to the square root of their masses (as would be expected for an axial electric field). 


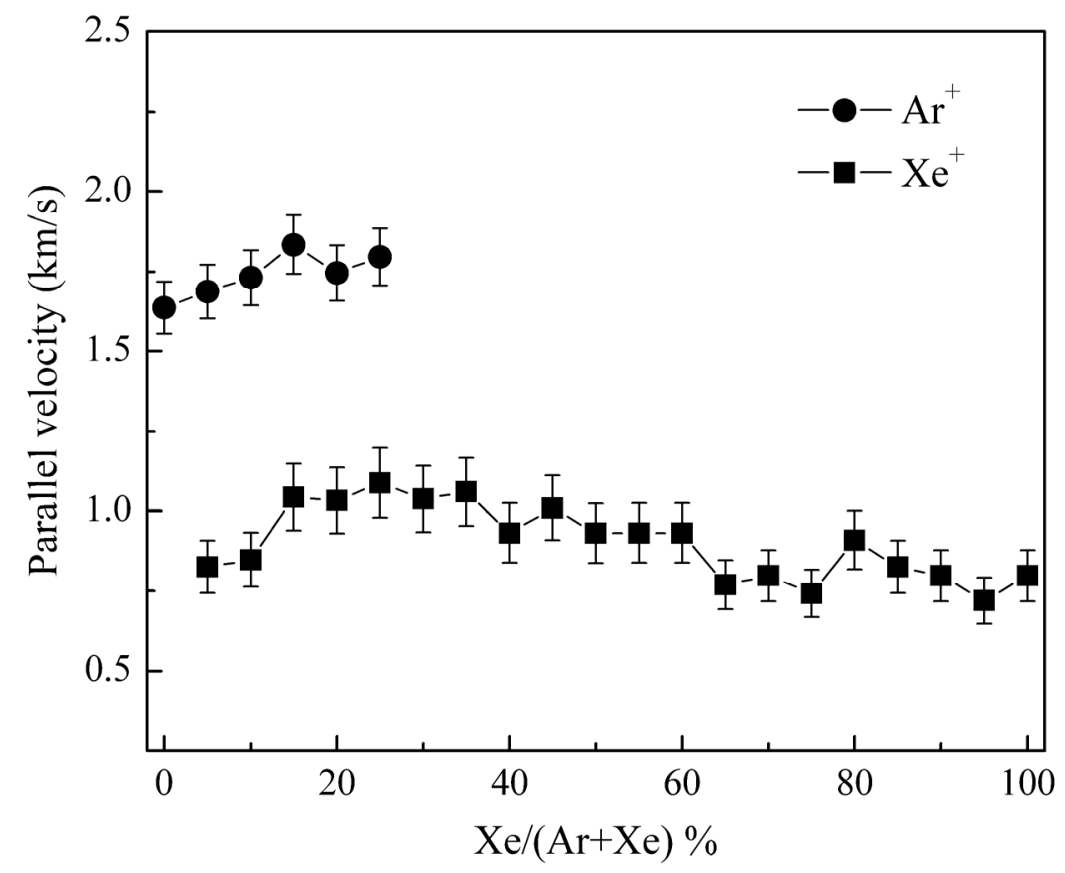

Figure 5.20. Parallel ion flow speeds in HELIX at $z=126 \mathrm{~cm}$ versus xenon fraction.

Further downstream, at $z=146 \mathrm{~cm}$ in HELIX ( $4 \mathrm{~cm}$ upstream of the HELIXLEIA junction), bimodal argon ivdfs are observed in Ar/Xe plasmas. The argon ivdfs are similar to those found in pure argon plasma (see Figure 5.9), with the fast group having a parallel velocity of $6.7 \mathrm{~km} / \mathrm{s}$ at $0 \%$ xenon fraction. The increase in parallel argon ion velocity between $z=126 \mathrm{~cm}$ and $z=146 \mathrm{~cm}$ is equivalent to an increase in parallel kinetic energy from 0.7 to $9.4 \mathrm{eV}$. This gain in parallel kinetic energy cannot be fully explained by conversion of upstream perpendicular energy into downstream parallel kinetic energy due to magnetic moment conservation (at $z=146 \mathrm{~cm}$ the magnetic field lines are no longer parallel but are slightly divergent). As previously mentioned, another possibility could be the conversion of azimuthal flow kinetic energy into parallel flow energy. However, magnetic moment conservation and the available flow kinetic energy could only provide, at most, a $2 \mathrm{eV}$ change in energy [21]. Therefore, a localized electric field between these two locations is implied. Unfortunately, the geometry of HELIX does not permit probe measurements at this location. Since the electron temperature is unlikely to vary much over $20 \mathrm{~cm}$, a rough estimate of the individual and system ion speeds (see Figure 5.21) based on the electron temperature measurements at $z=126 \mathrm{~cm}$ shows that at 
$z=146 \mathrm{~cm}$, the fast ion group is supersonic $\left(\sim 1.7 c_{\mathrm{Ar}}\right)$; consistent with an EDL between $z$

$=126 \mathrm{~cm}$ and $z=146 \mathrm{~cm}$ and subsequent ion acceleration.
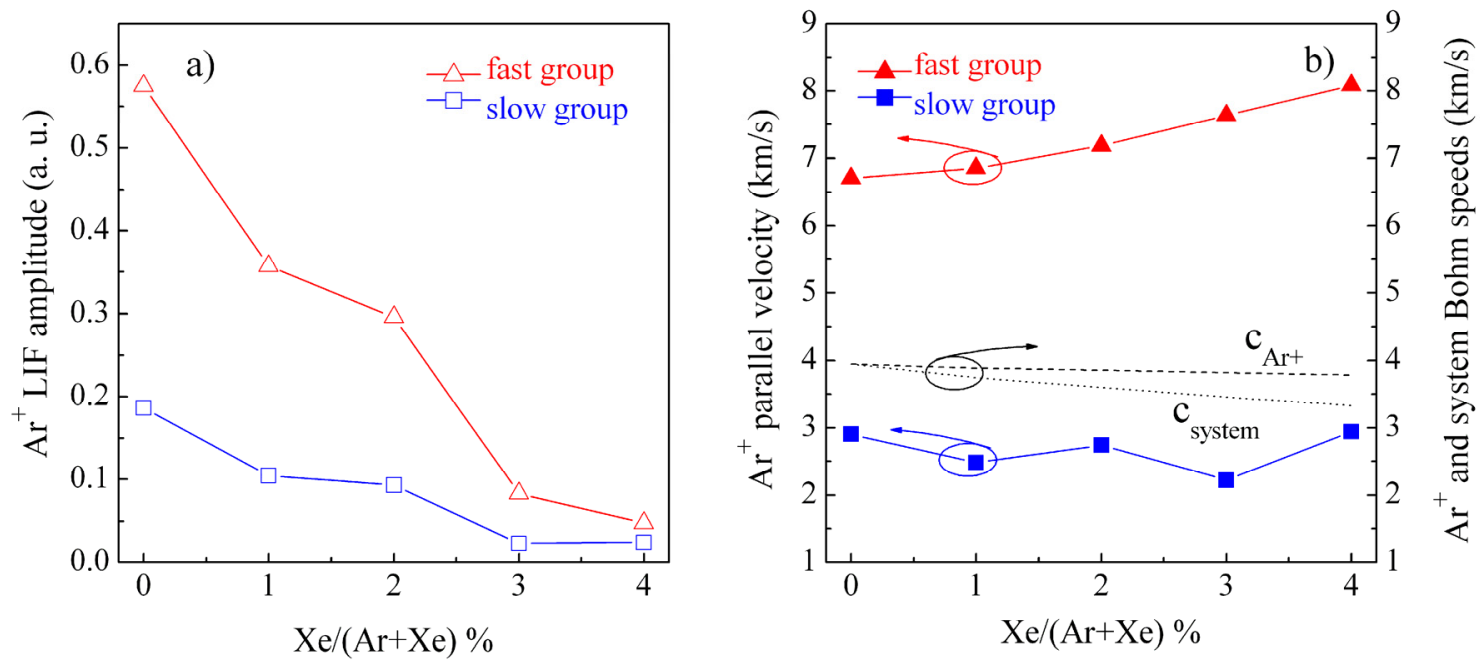

Figure 5.21. At $z=146 \mathrm{~cm}$ in HELIX, the $\mathrm{Ar}^{+}$LIF amplitude a) and parallel flow speed b) as function of small changes in the xenon fraction. Rf power was $750 \mathrm{~W}, B_{H}=700 \mathrm{G}, B_{L}=10 \mathrm{G}, p_{H}=1.3 \mathrm{mTorr}$, and $p_{L}=$ 0.14 mTorr. The $\mathrm{Ar}^{+}$and system sound speeds were calculated based on the measured electron temperature and electron density at $z=126 \mathrm{~cm}$.

At $z=146 \mathrm{~cm}$, the fast $\mathrm{Ar}^{+}$LIF amplitude decreases dramatically (more than a factor of ten) with increasing xenon fraction; disappearing entirely for xenon fractions greater than 4\% (see Figure 5.21(a)). Since at $z=126 \mathrm{~cm}$ the $\mathrm{Ar}^{+}$LIF signal is detectable up to xenon fractions of $\sim 20 \%$ and for xenon fractions of 0 to $4 \%$ the total pressure and the electron temperature are relatively constant, the change in $\mathrm{Ar}^{+}$density with increasing xenon fraction appears to be highly nonuniform along the HELIX axis (as shown in Figure 5.18, the LIF signal at $z=126$ is essentially constant for xenon fractions of $0-4 \%$ ). Previous experiments have shown that in pure argon plasmas, a decrease in $\mathrm{Ar}^{+}$density often corresponds to an increase in the potential difference across the EDL [42]. Therefore, these measurements may be indicative of modest changes in the strength of the EDL as the xenon fraction increases from 0 to $4 \%$.

The LIF collection optics mounted at $z=146 \mathrm{~cm}$ are capable of scanning a few centimeters along the HELIX axis. The argon ion beam velocities at $z=147 \mathrm{~cm}$ and $z=$ $148 \mathrm{~cm}$, shown with the measurements at $z=146 \mathrm{~cm}$ in Figure 5.22, are slightly larger $(\sim 2-5 \%)$ and exhibit the same dependence on xenon fraction as the $z=146 \mathrm{~cm}$ measurements, i.e. an increase of $\sim 1 \mathrm{~km} / \mathrm{s}$ over a $4 \%$ change in xenon fraction. This 
slight increase in the parallel flow velocity is consistent with the parallel acceleration expected for magnetic field divergence effects.

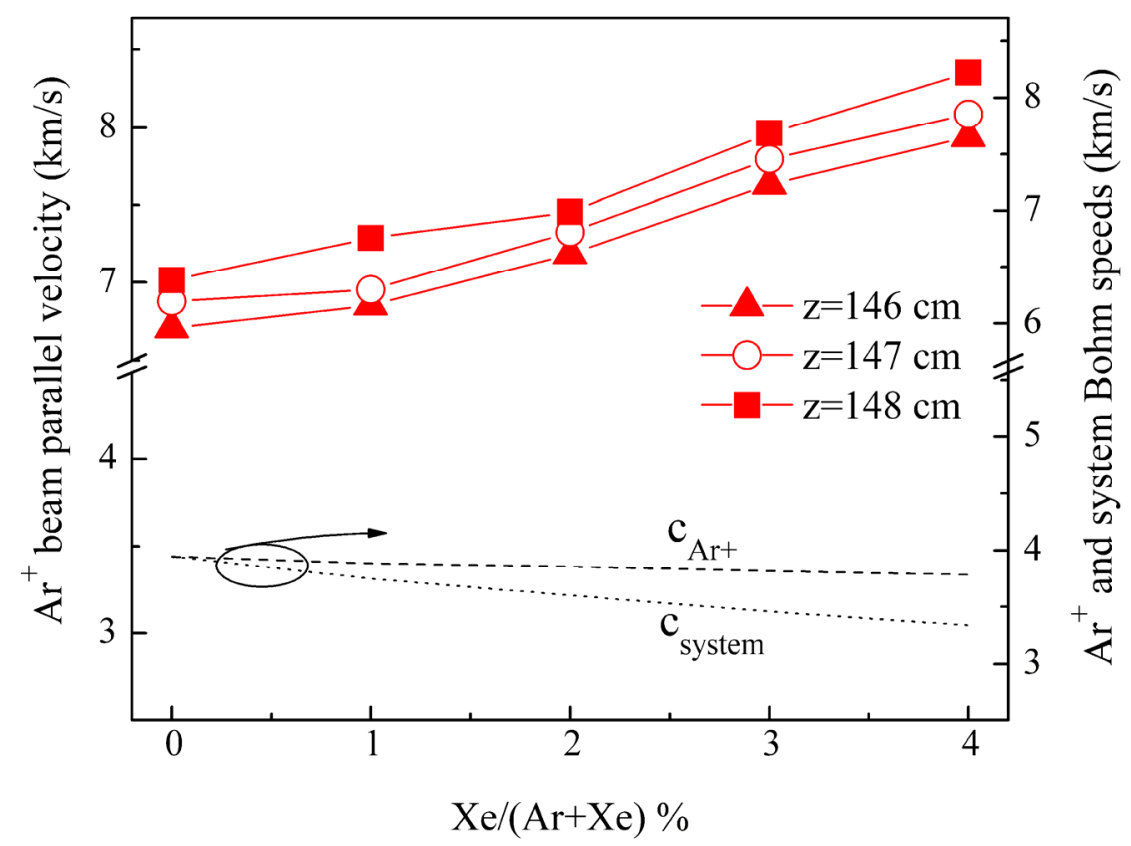

Figure 5.22. Fast $\mathrm{Ar}^{+}$population parallel velocity as function of xenon fraction at three locations close to HELIX-LEIA junction. The $\mathrm{Ar}^{+}$and the system Bohm speeds were calculated based on the measured electron temperature and electron density at $z=126 \mathrm{~cm}$.

As a function of xenon fraction, the $\mathrm{Ar}^{+}$and $\mathrm{Xe}^{+}$ivdfs deep in the helicon source are unimodal. Their axial velocities are subsonic and the ratio of their speeds is inversely proportional to square root of their relative masses. Towards the end of HELIX, the $\mathrm{Ar}^{+}$ ivdf has a bimodal structure, with a fast population presumably accelerated by an upstream EDL and a slow moving background ion population. The parallel velocity of the fast $\mathrm{Ar}^{+}$population is supersonic relative to the argon and total system sound speeds for xenon fractions less than $4 \%$. Increasing the xenon fraction substantially reduces the $\mathrm{Ar}^{+}$density and slightly increases the fast group axial velocity.

\subsection{1.b. Effect of Ar/Xe Ratio on Argon IVDF in the Expansion Region}

HELIX-LEIA is a freely expanding plasma system having a physical expansion ratio (the diffusion chamber cross-sectional area divided by the plasma source tube crosssectional area) of $\sim 1.8$. Since the gas inlet port is at the end of HELIX opposite LEIA and 
the vacuum pumping port at the end of LEIA, the neutral pressures in HELIX are ten times larger than in LEIA. As noted previously, by increasing the xenon fraction for a constant total mass flow rate, the total neutral pressure in HELIX increases from 1.3 mTorr at $0 \%$ xenon to 1.5 mTorr at $100 \%$ xenon. The corresponding change in LEIA pressure is from 0.14 mTorr to $0.16 \mathrm{mTorr}$ for pure argon and pure xenon, respectively. $\mathrm{Ar}^{+}$LIF measurements as a function of xenon fraction were obtained in LEIA with the scanning probe at $z=169 \mathrm{~cm}$ (point $\mathrm{P}_{1}$ in Figure 4.24). For these measurements, the operating parameters were the same as for the HELIX investigations: rf power of $750 \mathrm{~W}$, HELIX magnetic field strength of $700 \mathrm{G}$, and LEIA magnetic field strength of $10 \mathrm{G}$. The bimodal character of the argon ivdf observed in HELIX just upstream of the HELIXLEIA junction is also observed in LEIA. In Figure 5.23, similar to what was observed in HELIX, the LIF intensity of the fast $\mathrm{Ar}^{+}$population decreases with xenon fraction and disappears completely for xenon fractions greater than 3\% (in HELIX the signal disappeared for xenon fractions greater than $4 \%$ ). Since the LIF detection system in LEIA is different than in HELIX, direct comparison of LIF signal amplitudes is inappropriate. However, the relative values do provide some insight into the dependence of the fast and slow ion populations on the xenon fraction. At the end of HELIX, the fast ion population LIF signal is much larger than the slow ion population LIF signal (see Figure 5.21 (a)); $75 \%$ of the total population for pure argon and $81 \%$ of the total population for a $3 \%$ xenon fraction. In LEIA the fast ion population is a small fraction of the total ion population: $15 \%$ for pure argon and only $4 \%$ for a $3 \%$ xenon fraction. The parallel flow speed of the fast population in LEIA is larger $(\sim 10.8 \mathrm{~km} / \mathrm{s})$ than the parallel flow speed at the end of HELIX $(\sim 8 \mathrm{~km} / \mathrm{s})$. Conversely, the parallel flow speed of the slow population $(1.4 \mathrm{~km} / \mathrm{s})$ is smaller than the corresponding HELIX value of $2.5 \mathrm{~km} / \mathrm{s}$. The parallel flow speeds of the fast and slow ion populations in LEIA are unaffected by the small change in xenon fraction. These observations are consistent with different origins of the two ion populations observed in LEIA: the slow ions are a background ion population created locally and the fast ions are an ion population created upstream in the source and subsequently accelerated into the LEIA chamber. Under these assumptions, the decrease in the fraction of the fast ion population at $z=169 \mathrm{~cm}$ is entirely consistent with metastable quenching of the fast ion population. Since LIF only detects ions in the $3 \mathrm{~d}$ 
${ }^{2} \mathrm{G}_{9 / 2}$ metastable state, only a small fraction of the fast ion population is detectable by LIF. Taking the upper limit of the quenching cross-section for collisions of the $3 \mathrm{~d}^{2} \mathrm{G}_{9 / 2}$ state with ground state neutral argon to be $1 \times 10^{-14} \mathrm{~cm}^{2},{ }^{44}$ the resultant mean free path (mfp) is $\sim 17 \mathrm{~cm}$, one and a half times the distance between the likely EDL location and observation point in LEIA. Thus, only $\sim 20 \%$ of the fast ions in the $3 \mathrm{~d}^{2} \mathrm{G}_{9 / 2}$ metastable state accelerated by the EDL at the end of HELIX will survive and be detected in LEIA. The effect of metastable quenching on LIF measurements of ion beams was recently demonstrated in a series of combined RFEA and LIF experiments in LEIA [18].
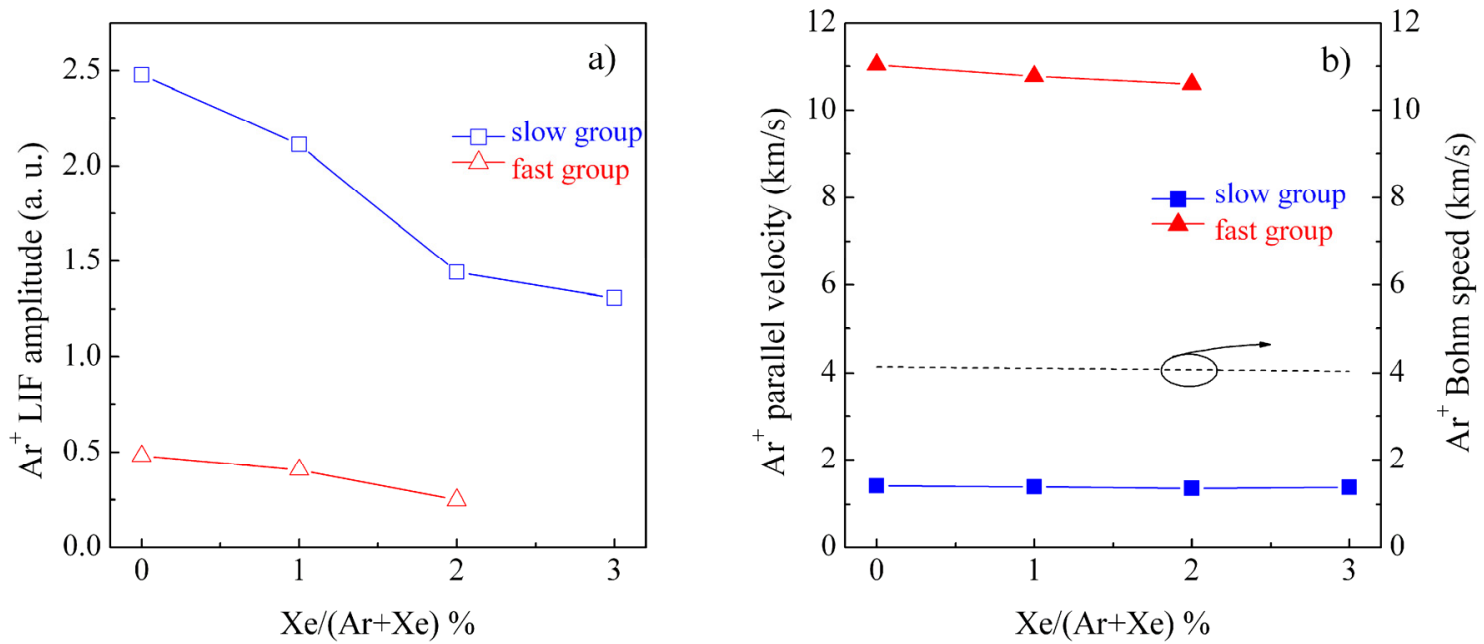

Figure 5.23. a) $\mathrm{Ar}^{+}$LIF amplitude and b) parallel flow speed as function of xenon fraction at $z=169 \mathrm{~cm}$ in LEIA. Operating conditions: $B_{H}=700 \mathrm{G}, B_{L}=10 \mathrm{G}, p_{H}=1.3$ mTorr, $p_{L}=0.14 \mathrm{mTorr}$, and $\mathrm{P}_{\mathrm{rf}}=750 \mathrm{~W}$. The argon ion Bohm speed was calculated based on the measured electron temperature at $z=169 \mathrm{~cm}$.

The decrease in LIF signal with increasing xenon fraction results from fewer argon ions in the $3 \mathrm{~d}^{2} \mathrm{G}_{9 / 2}$ metastable state being generated in the source (recall that an increasing xenon fraction depletes the eedf of higher energy electron (see Figure 5.2) and thereby reduces the rate of argon ion metastable creation). Although the cross-sections for $\mathrm{Ar}^{+}{ }_{-}$Xe quenching collisions are unknown, they are probably negligible for such small xenon fractions.

In addition to the plasma density and electron temperature downstream of HELIX-LEIA junction (Figure 5.4), the plasma potential plays an important role in ion acceleration. The measured floating potential in LEIA $(\sim 20 \mathrm{~cm}$ downstream HELIXLEIA junction) and the corresponding plasma potential measured and calculated according to Eq. (4.9) are shown in Figure 5.24. The negative floating potential increases 
dramatically, becomes more positive, with the addition of a few percent of xenon and saturates at a value of $\sim-7 \mathrm{~V}$ for xenon fractions greater than $40 \%$. However, the large ionization cross section of xenon causes a sharp decrease in the electron temperature and therefore the plasma potential is roughly constant at $\sim 22 \mathrm{~V}$ for xenon fractions of $0-10 \%$. For larger xenon fractions, the plasma potential decreases linearly to $\sim 17 \mathrm{~V}$.
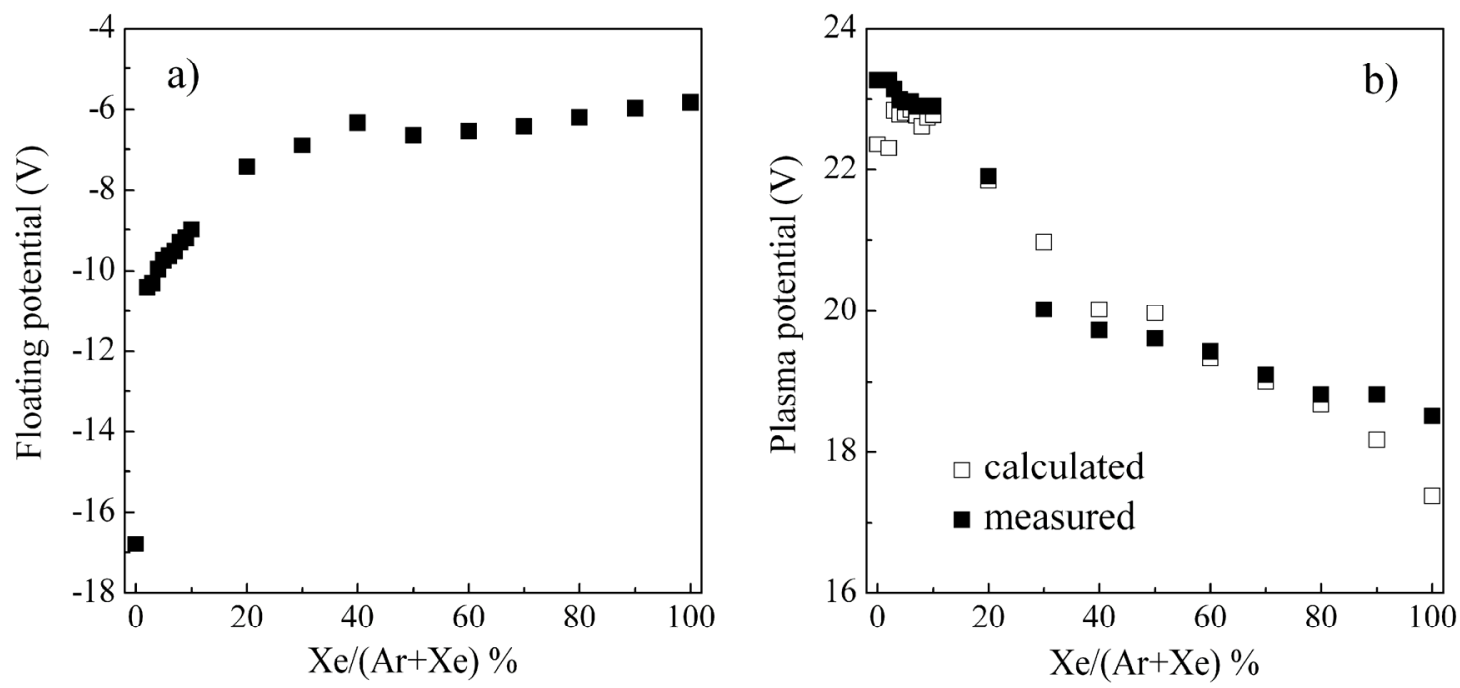

Figure 5.24. a) Measured floating potential and b) measured and calculated plasma potential $20 \mathrm{~cm}$ downstream of the HELIX-LEIA junction versus xenon fraction.

A parallel ion flow speed of $10.8 \mathrm{~km} / \mathrm{s}$ in LEIA corresponds to a parallel kinetic energy of $\sim 24 \mathrm{eV}$. Given the $\sim 13 \mathrm{eV}(8 \mathrm{~km} / \mathrm{s})$ kinetic energy measured at the end of HELIX, these measurements suggest that the ions gain an additional $\sim 11 \mathrm{eV}$ as they travel through the divergent magnetic field region from $z=146 \mathrm{~cm}$ (HELIX) to $z=169$ $\mathrm{cm}$ (LEIA). Consistent with this interpretation is the observation that for small changes in the xenon fraction ( 0 to $4 \%$ ) the plasma potential in LEIA is roughly constant, i.e., there is no change in the total potential drop across the EDL (unfortunately, measurement of the plasma potential just upstream the EDL is not possible in the HELIX-LEIA system but based on $\mathrm{Ar}^{+}$LIF measurements in HELIX and the energy balance equation the estimated upstream plasma potential is $\sim 43-46 \mathrm{~V}$ for these conditions). Note also that the magnetic field ratio was held constant in these experiments. Thus, it appears that a small addition of xenon to argon plasma in the range of pressure where the EDL is present does not alter the parallel speed of the supersonic $\left(\sim 2.6 c_{\mathrm{Ar}+}\right)$ fast ion group and that the total 
energy gain arises from a combination of acceleration in the EDL $\left(12-14 \mathrm{eV} \cong 2 k_{B} T_{e}\right)$ and adiabatic expansion in the divergent magnetic field $(\sim 11-13 \mathrm{eV})$.

\subsubsection{Xenon IVDF in Ar-Xe mixture}

Particle-in-cell (PIC) simulations of multiple-ion plasma sheaths suggest that the ratio of the individual ion collisional mean free paths is the most important parameter for determining the relative ion acceleration in the sheath. ${ }^{45}$ In a two-ion species plasma, under the assumption of equal ion fluxes at the sheath edge, the PIC simulations showed that ion species with the larger collision mean free path enters the sheath with a velocity larger than its own Bohm velocity. In the limit that one ion flux is much less than the other, the dominant ion has its own Bohm velocity at the sheath edge. The total momentum transfer cross section for $\mathrm{Ar}^{-\mathrm{Ar}^{+}}$collisions at energies under $10 \mathrm{eV}(\sigma \approx 1.56$ $\times 10^{-14} \mathrm{~cm}^{2}{ }^{46}$ ) results in an argon ion mean free path of $\lambda_{A r}=2.6 / p$ (with the mean free path in $\mathrm{cm}$ and the neutral pressure $p$ in mTorr). Similarly, for a total momentum transfer

cross section for Xe-Xe ${ }^{+}$collisions of $\sigma \approx 2.34 \times 10^{-14} \mathrm{~cm}^{2},{ }^{47}$ the xenon ion mean free path is $\lambda_{X e}=1.7 / p$. Although in Ar-Xe plasma, ion-neutral collisions between species may occur, the minimum and maximum mean free path values are given by the pure xenon and pure argon limits. Under the condition of constant flow rate, the pressure in the HELIX-LEIA system for pure xenon is $\sim 15 \%$ more than for pure argon. Thus, the ion mean free path in pure xenon plasma is approximately two times smaller than the argon ion mean free path in pure argon plasma.

To examine the effect of light ion mass doping, LIF measurements of the xenon ivdf were obtained as a function of argon fraction in HELIX at $z=126 \mathrm{~cm}$ and $z=146$ $\mathrm{cm}$. The plasma conditions were the same as for the xenon fraction experiments, i.e., the rf power was $750 \mathrm{~W}$, rf driving frequency was $9.5 \mathrm{MHz}$, and the magnetic field strengths in the source and expansion chamber were $700 \mathrm{G}$ and $10 \mathrm{G}$, respectively, except the total mass flow rate was maintained at $8 \mathrm{sccm}$; a mass flow rate for which a quiescent plasma could be maintained. The total neutral pressure in HELIX decreased from 1.3 mTorr for $0 \%$ Ar to 1.1 mTorr at $87 \%$ Ar. For argon fractions less than $80 \%$, ion density was dominated by the xenon ion (see Figure 5.17). 

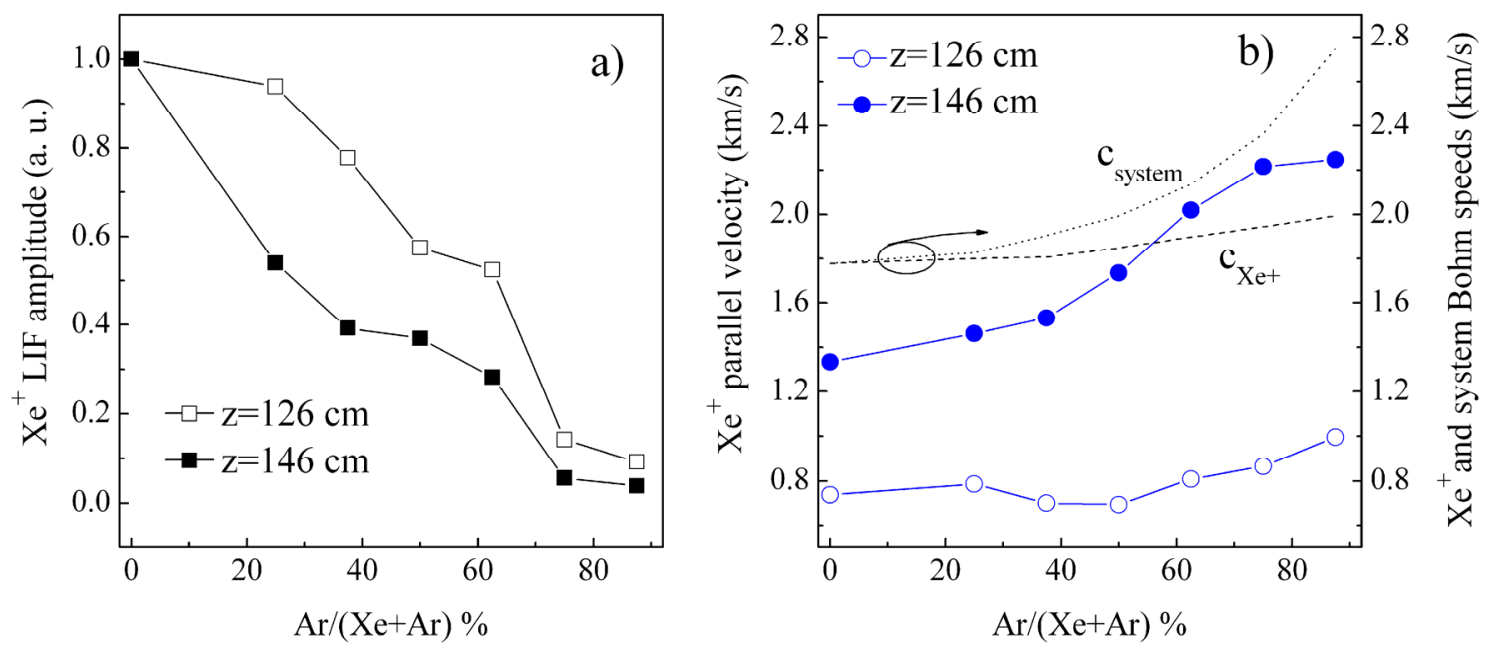

Figure 5.25. a) $\mathrm{Xe}^{+}$LIF amplitude and b) $\mathrm{Xe}^{+}$parallel flow speed at $z=126 \mathrm{~cm}$ and $z=146 \mathrm{~cm}$ in HELIX versus argon fraction. Operating conditions were: $\mathrm{P}_{\mathrm{rf}}=750 \mathrm{~W}, f=9.5 \mathrm{MHz}, B_{H}=700 \mathrm{G}, B_{L}=10 \mathrm{G}, p_{H}$ $(100 \% \mathrm{Xe})=1.3 \mathrm{mTorr}, F=8 \mathrm{sccm}$. The $\mathrm{Xe}^{+}$and system Bohm speeds were calculated based on electron temperature and electron density measurements at $z=126 \mathrm{~cm}$.

Since the $\mathrm{Xe}^{+} 5 \mathrm{~d}{ }^{4} \mathrm{D}_{7 / 2}$ metastable state formation is dominated by electron collisions with the $\mathrm{Xe}^{+}$in the ground state, the LIF signal is proportional to the electron density, the xenon neutral density, and the $\mathrm{Xe}^{+}$density. As can be seen in Figure 5.25 (a), at both $z=126 \mathrm{~cm}$ and $z=146 \mathrm{~cm}$, the LIF intensity, and implicitly the metastable $\mathrm{Xe}^{+}$ density, decreases almost linearly with increasing argon fraction. For an argon fraction of $87 \%$, the $\mathrm{Xe}^{+}$LIF signal is still detectable but has decreased by more than $90 \%$ with respect to the pure xenon case. Also shown in Figure 5.25 (b) are the parallel $\mathrm{Xe}^{+}$flow speeds and the $\mathrm{Xe}^{+}$and system sound speeds. Deep in the source, at $z=126 \mathrm{~cm}$, the $\mathrm{Xe}^{+}$ parallel flow is subsonic and insensitive to increasing argon fraction. In contrast to the $\mathrm{Ar}^{+}$ivdf, which at $z=146 \mathrm{~cm}$ exhibited a bimodal structure as a result of EDL formation, the $\mathrm{Xe}^{+}$ivdf is unimodal. Because xenon is more than three times heavier than argon, acceleration through an EDL identical to that found in the pure argon plasmas would yield a relative $\mathrm{Xe}^{+}$drift velocity of

$$
v_{X e^{+}} / v_{A r^{+}}=\left(M_{A r^{+}} / M_{X e^{+}}\right)^{1 / 2}=(40 / 131)^{1 / 2} \cong 1 / \sqrt{3} .
$$

Given the high velocity resolutions achievable with LIF $(\sim 50 \mathrm{~m} / \mathrm{s})$ dual xenon and argon LIF may provide a method of accurately measuring weak electric fields in plasma. The above relationship was confirmed with the $z=126 \mathrm{~cm}$ LIF measurements (see Figure 
5.26 (b)). The flow speed difference shown in Figure 5.26 (a) is consistent with a slight potential gradient of $\sim 5 \mathrm{mV} / \mathrm{cm}$ along the axis of the system (assuming zero parallel flow speed at the downstream edge of the rf antenna).
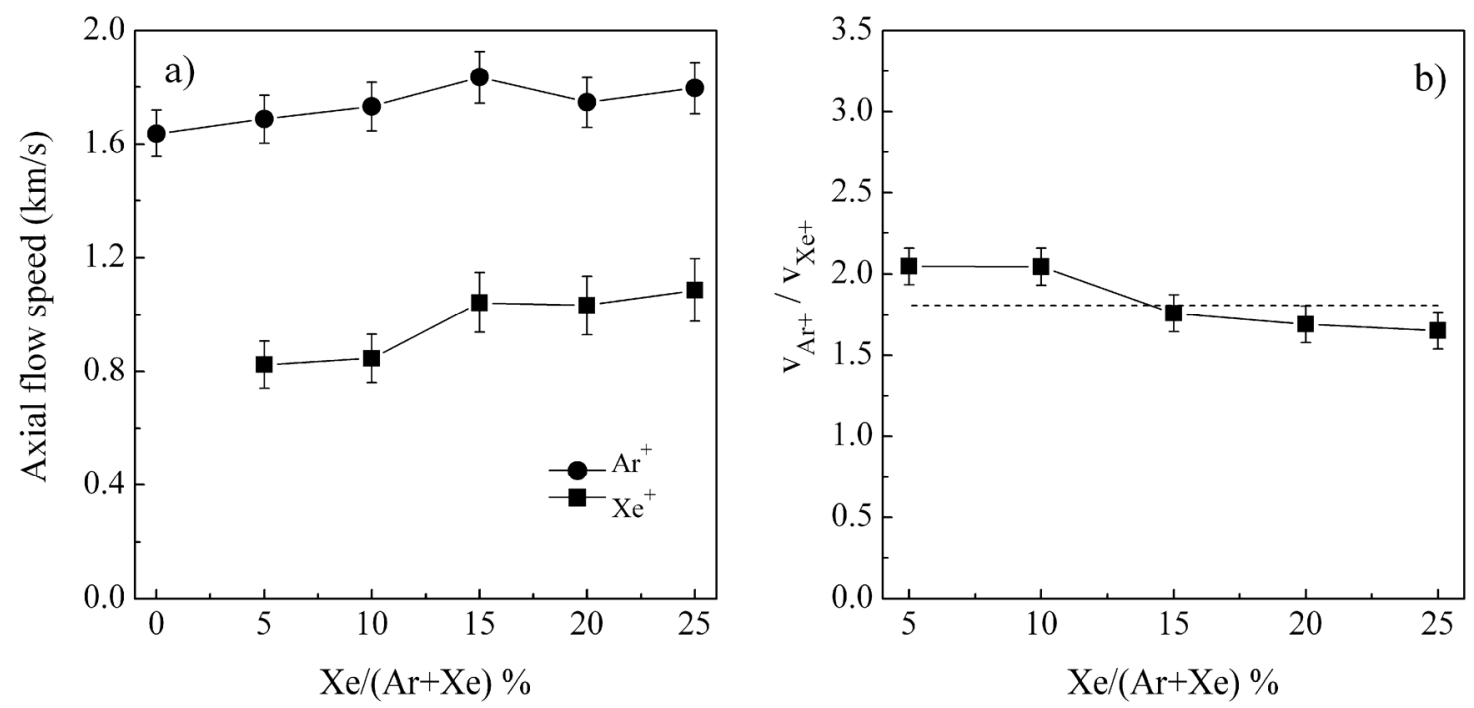

Figure 5.26. a) $\mathrm{Xe}^{+}$and $\mathrm{Ar}^{+}$axial flow speeds in Ar-Xe plasma at $z=126 \mathrm{~cm}$ from LIF data as a function of xenon fraction (only $\mathrm{Ar} / \mathrm{Xe}$ mixture range where both ivdfs are detected is shown). b) the ratio of experimentally determined parallel flow speeds and the square root of mass ratio, confirming Eq. (5.10)

At the end of HELIX $(z=146 \mathrm{~cm})$ for a drift velocity of $8 \mathrm{~km} / \mathrm{s}$ for $\mathrm{Ar}^{+}$, Eq. (5.10) would predict a $4.6 \mathrm{~km} / \mathrm{s}$ drift velocity for $\mathrm{Xe}^{+}$. In spite of lower gas pressure operation, no $\mathrm{Xe}^{+}$beam has been observed. At $z=146 \mathrm{~cm}$ the peak velocity of the unimodal ivdf gradually increases with increasing argon percentage in the mixture, approaching the system sound speed for an argon fraction of $\sim 60 \%$. For an argon fraction of $87 \%$, the $\mathrm{Xe}^{+}$parallel flow speed reaches $2.2 \mathrm{~km} / \mathrm{s}$. For an argon-xenon mixture, addition of a light mass gas (Ar) to a heavier gas (Xe) appears to increase the heavy ion axial flow velocity. As will be shown in the next section, a similar effect is observed in argon-helium mixture: addition of a light mass gas (He) to a heavier gas (Ar) shifts the heavy ion parallel speed to higher values.

In the absence of any xenon ion beam observations, it is not possible to draw any conclusions regarding the effects of argon fraction on EDL formation, if any, in xenon plasmas. For smaller helicon sources, other groups have reported that neutral pressure is a key factor in EDL formation in heavy weight gases [27,29]. Bimodal RFEA-determined 
ivdfs with one component accelerated to supersonic speeds have been reported for xenon pressures of 0.07 mTorr by Charles [27] and 0.09 mTorr by Shamrai [29]. Those pressures are one order of magnitude lower than the pressure used in these experiments. Attempts to lower the operating pressure led to unstable plasma conditions and unreliable LIF measurements.

In the absence of an EDL, it is typically assumed that as electrons stream out along the magnetic field lines in an open magnetic geometry such as HELIX-LEIA, an ambipolar electric field builds up and the ions are dragged out by the ambipolar field. ${ }^{48}$ The ionization of neutrals along the axis of the system, radial transport, ion-electron recombination, and neutral drag (due to ion-neutral collisions) can all modify the ion flow along the magnetic field lines. Radial transport effects are particularly important for HELIX-LEIA because for the first $60 \mathrm{~cm}$ of the source downstream of the rf antenna, the source walls are electrically non-conductive and for the next $90 \mathrm{~cm}$ the source has grounded walls. An estimate of the ambipolar electric field was obtained in the source through LIF data obtained at $z=126 \mathrm{~cm}$. To calculate the magnitude of the axial electric field in HELIX, two sets of Xe $\mathrm{e}^{+}$LIF data were obtained at $z=126 \mathrm{~cm}$ and $z=146 \mathrm{~cm}$ (locations A and B in Figure 3.3). Most of the operating parameters were the same as for previous experiments, i.e., $9.5 \mathrm{MHz}$ driving frequency, $700 \mathrm{G}$ and $10 \mathrm{G}$ magnetic field strengths in HELIX and LEIA, respectively. Instead of varying the mass flow rate, which introduces significant changes in the ion mean free path, the pressure was held constant at $1.3 \mathrm{mTorr}$ for pure xenon and the input rf power was varied from 350 to $750 \mathrm{~W}$. As shown in Figure 5.27 (a), the $\mathrm{Xe}^{+}$parallel flow speeds at both locations were subsonic and were not affected by changes in the rf power level. The measurements clearly show an increase in xenon ion flow speed as the ions enter in the weakening magnetic field region at the end of the source. Based on $\sim 750 \mathrm{~m} / \mathrm{s}$ and $\sim 1400 \mathrm{~m} / \mathrm{s}$ parallel flow speeds at $z=126 \mathrm{~cm}$ and $z=146 \mathrm{~cm}$, respectively, and the $20 \mathrm{~cm}$ axial separation of the observation points, the calculated axial potential gradient is $\sim 50 \mathrm{mV} / \mathrm{cm}$, ten times larger than the field value obtained at $z=126 \mathrm{~cm}$ from the combined $\mathrm{Ar}^{+}$and $\mathrm{Xe}^{+} \mathrm{LIF}$ measurements. 

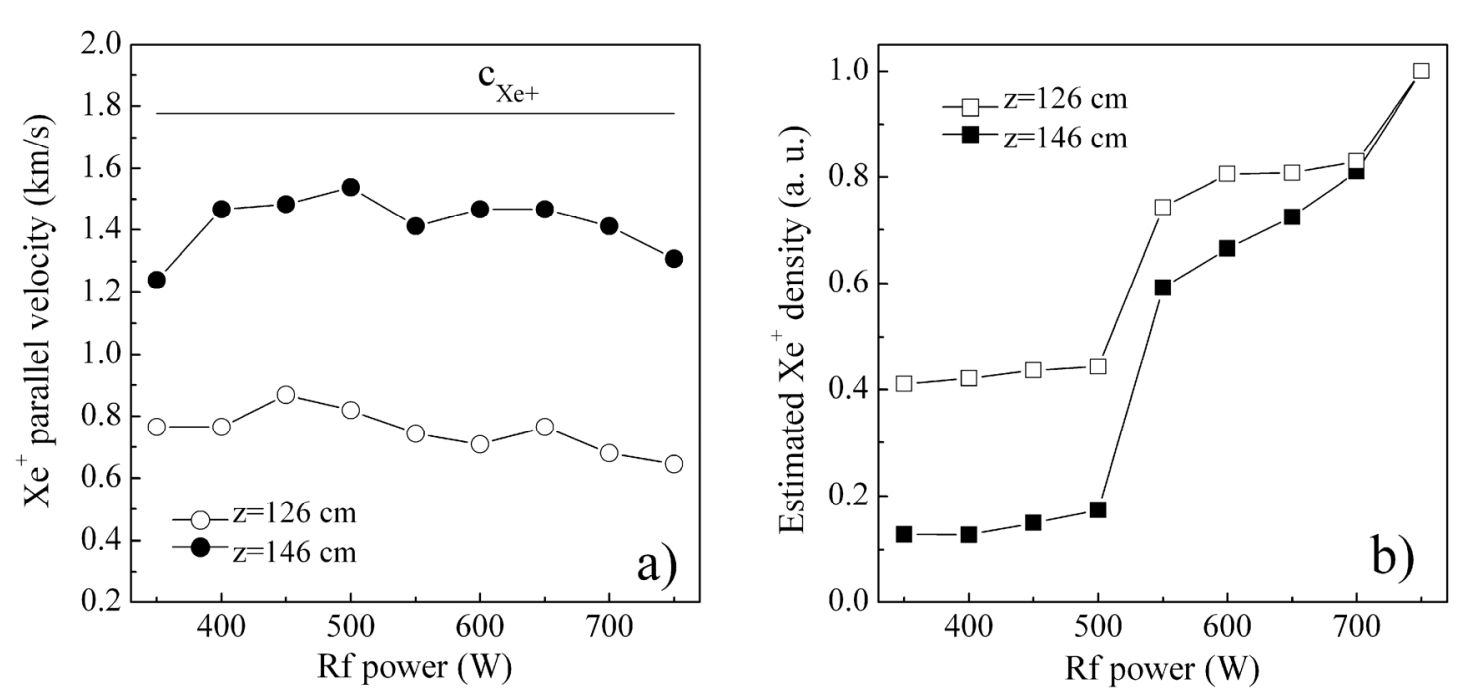

Figure 5.27. a) $\mathrm{Xe}^{+}$parallel flow speed in HELIX and b) the estimated $\mathrm{Xe}^{+}$density from the LIF signal amplitude versus input rf power. Operating conditions: $f=9.5 \mathrm{MHz}, B_{H}=700 \mathrm{G}, B_{L}=10 \mathrm{G}, p_{H}=1.3$ mTorr.

The square root of the $\mathrm{Xe}^{+} \mathrm{LIF}$ signal amplitude as a function of rf power is shown in Figure 5.27 (b). Since the electron temperature is roughly constant for the different input rf powers, the square root of LIF signal amplitude is a qualitative indicator of the trend $\mathrm{Xe}^{+}$density. Due to the inherent divergence of the injected laser beam and different LIF collection optics used at those two locations (larger lens diameter at $z=126$ $\mathrm{cm}$ ), the LIF signal amplitude is smaller at $z=146 \mathrm{~cm}$. Therefore, to have a clear picture of the ion density evolution with input rf power, the LIF signals at both locations were normalized to their maximum values (corresponding to highest power level). With increasing rf power, a significant jump in ion density at $550 \mathrm{~W}$ is observed at both locations. This density jump corresponds to a discharge mode change from electrostatic (E mode) to inductive (H mode). ${ }^{49,50}$ A second density jump that might be associated with an inductive to helicon (W mode) mode transition is also observed around $750 \mathrm{~W}$. The similarity in the ion density trends at both two axial locations and the fact that more than $100 \mathrm{~cm}$ downstream from the antenna the LIF signal still "feels" the changes in rf coupling modes provides further support for the use of the LIF signal as a qualitative indicator of ion density. Note that these datasets were acquired independently, i.e., after the power scan was performed at $z=126 \mathrm{~cm}$, a second power scan was performed at $z=$ 
$146 \mathrm{~cm}$. Thus, the matching network settings had to be completely readjusted to minimize the reflected power for both experiments.

\subsubsection{Argon IVDF in Ar-He mixture}

To further investigate the influence of a light mass ion on a heavier ion species velocity distribution, a series of experiments were performed in an argon-helium mixture plasma. The discharge was ignited in pure argon plasma at a total mass flow rate of 10 sccm. Helium and argon flow rates were then adjusted in a controlled manner so that the total mass flow rate was kept constant and the helium fraction was increased up to $80 \%$. The same source parameters for the Ar-Xe plasma experiments were used: rf power of $750 \mathrm{~W}$, the rf driving frequency of $9.5 \mathrm{MHz}$, HELIX magnetic field strength of $700 \mathrm{G}$, and LEIA magnetic field strength of $10 \mathrm{G}$. As shown in Figure 5.28 (a), by increasing the helium fraction, the pressure in HELIX drops significantly from 1.3 mTorr in pure argon down to $0.2 \mathrm{mTorr}$ for a helium fraction of $80 \%$. Further increase of the helium fraction was not possible since the discharge could not be maintained at $750 \mathrm{~W}$ of input rf power and such low pressure. As an effect of the decreasing pressure and higher helium ionization potential, Langmuir probe measurements taken $20 \mathrm{~cm}$ downstream from the antenna show a two fold increase in the electron temperature with increasing helium fraction; from $\sim 7 \mathrm{eV}$ for pure argon up to $\sim 14 \mathrm{eV}$ for a helium fraction of $80 \%$. Over the same helium fraction range, the electron density drops by more than three orders of magnitude, from $1.14 \times 10^{11} \mathrm{~cm}^{-3}$ to $6.5 \times 10^{7} \mathrm{~cm}^{-3}$. Computed ion densities using Eq. (4.6), the measured electron density, and the calculated ionization rate coefficients for each ion species suggest that argon ions far outnumber helium ions in these mixed gas plasmas. As can be seen in the inset on Figure 5.28 (b), the argon ions dominate the plasma density even for a 80/20 He/Ar mixing ratio. The much larger argon ion density results from the large differences in the helium and argon ionization potentials $(24.58 \mathrm{eV}$ for helium versus $15.76 \mathrm{eV}$ for argon) and the ionization cross-sections (peak value of $3.5 \times 10^{-17} \mathrm{~cm}^{2}$ for helium versus $2.8 \times 10^{-16} \mathrm{~cm}^{2}$ for argon). 

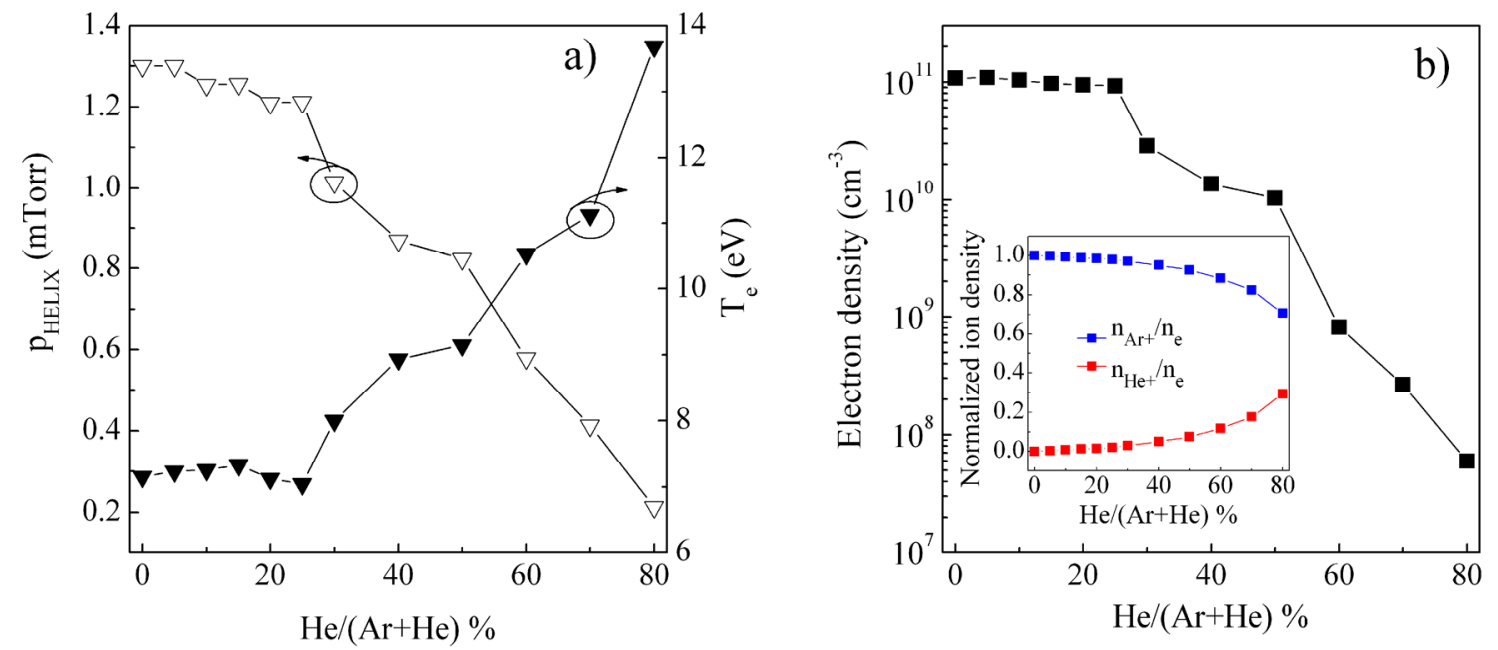

Figure 5.28. a) Total neutral pressure (open symbol) and the electron temperature (filled symbol) in HELIX plasmas a function of helium fraction; b) electron density inferred from Langmuir probe measurements versus helium fraction in HELIX; in the inset, the normalized partial ion densities. Operating conditions: $P_{r f}=750 \mathrm{~W}, f=9.5 \mathrm{MHz}, B_{H}=700 \mathrm{G}, B_{L}=10 \mathrm{G}$.

LIF measurements of the $\mathrm{Ar}^{+}$ivdf at the end of HELIX (at $z=146 \mathrm{~cm}$ ) for different argon-helium compositions are presented in Figure 5.29. Up to helium fractions of $30 \%$, a bimodal $\mathrm{Ar}^{+}$ivdf comprised of fast and slow populations is observed. For helium fractions greater than $30 \%$, the $\mathrm{Ar}^{+} \mathrm{LIF}$ signal is buried in the noise and the ivdf could not be measured. The overall decrease of LIF signal (proportional to metastable $\mathrm{Ar}^{+}$density) and the shift toward higher speeds for the fast ion population with increasing helium fraction are immediately obvious in the measured ivdfs. 


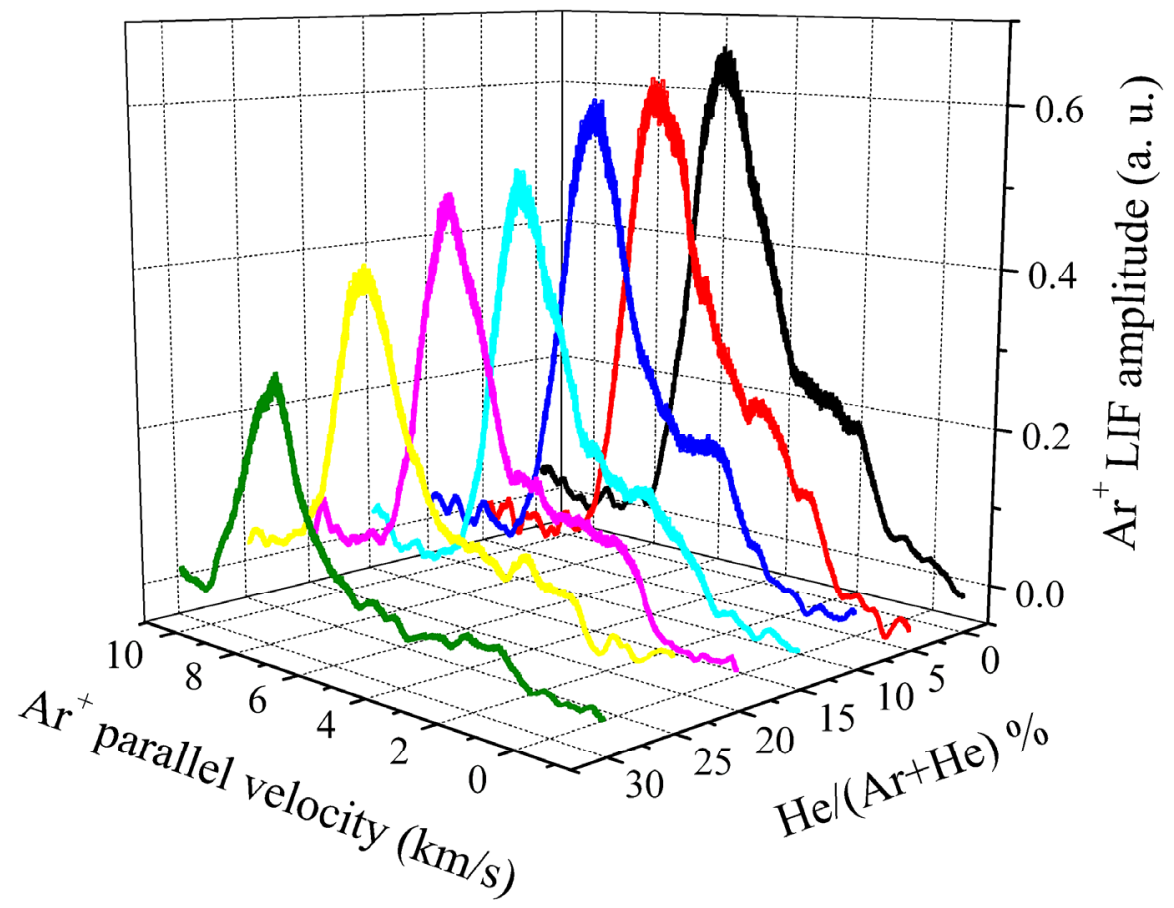

Figure 5.29. Effect of increasing helium fraction on the $\mathrm{Ar}^{+}$parallel ivdf in HELIX. Measurements were obtained on axis at $z=146 \mathrm{~cm}$.

These measurements directly contradict previous LIF observations in an electron cyclotron Ar-He plasma by Sadeghi et al. [44]. For a constant helium flow rate in those experiments, an increase in argon flow rate led not only to an overall decrease of the argon LIF signal but also to a decrease in the slow/fast LIF signal ratio as well. Another difference is the direction of the velocity shift: in that report, increasing the argon fraction shifted the distribution toward higher speeds. Our measurements show that reduction in the argon fraction (increase in helium fraction) leads to an increase in the parallel ion flow speed. Although the experiments differ in implementation (in our experiment the total gas flow is held constant whereas in the Sadeghi et al. work the flow of one gas was held constant while the flow rate for the second gas was varied) the total pressure ranges (0.2 - $1.3 \mathrm{mTorr}$ in our experiment versus 0.4 - 1 mTorr for Sadeghi's experiment) and gas composition ( $0-80 \%$ helium in our work versus $33-87 \%$ in Sadeghi's work) are similar. These quite different results for similar plasma parameters prompted a more complete analysis of the effects of increasing helium fraction on the $\mathrm{Ar}^{+}$ivdf. 

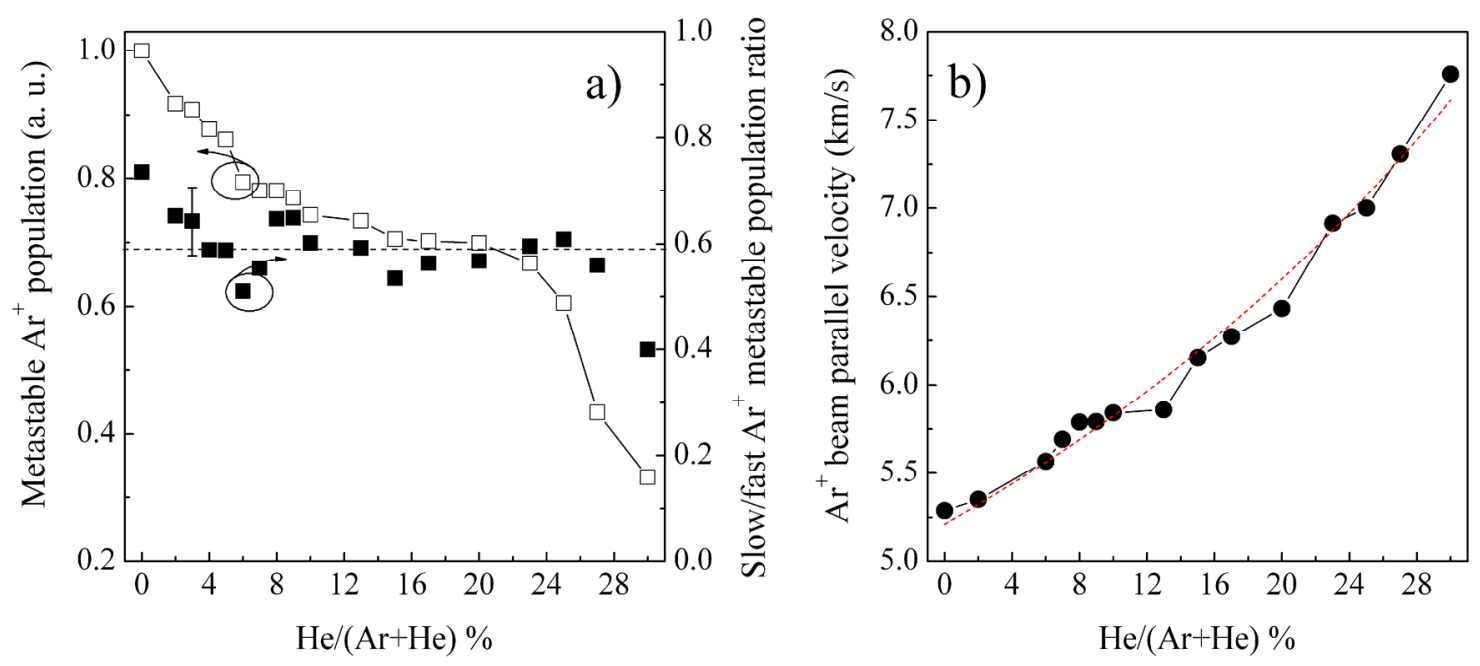

Figure 5.30. a) Dependence of the metastable $\mathrm{Ar}^{+}$population obtained from integration of the ivdfs and normalized to the pure Ar case (open symbols) and ratio of slow/fast LIF $\mathrm{Ar}^{+}$signals (filled symbols) versus helium fraction; b) parallel velocity of the fast ion population. The dashed red line is theoretical dependence $\sim[\alpha+\beta /(1-\gamma x)]$. Measurements obtained at $z=146 \mathrm{~cm}$ in HELIX.

As shown in Figure 5.30 (a), our experimental measurements show that the total $\mathrm{Ar}^{+}$metastable population (obtained by integration of the LIF signal) increases with increasing argon fraction, i.e., an increase in the helium fraction yields a decrease in the overall $\mathrm{Ar}^{+}$LIF signal. In spite of a $\sim 23 \%$ decrease in the total neutral pressure (from 1.3 mTorr at $0 \%$ helium fraction to $1 \mathrm{mTorr}$ at $30 \%$ helium fraction), for helium fractions of $0 \%$ to $30 \%$, the electron temperature is roughly constant at $\sim 7 \mathrm{eV}$ (see Figure 5.28 (a)). Thus, the calculated $\mathrm{Ar}^{+}$density suggests little variation in $\mathrm{Ar}^{+}$density over the same helium fraction range: a slight decrease from $1.1 \times 10^{11} \mathrm{~cm}^{-3}$ in pure argon to $9.4 \times 10^{10} \mathrm{~cm}^{-}$ ${ }^{3}$ at a helium fraction of $25 \%$, i.e., $15 \%$ decrease in $\mathrm{Ar}^{+}$density. In spite of the relative constancy of the electron temperature (which implies a constant excitation rate coefficient for the stepwise production channel of the $\operatorname{Ar}^{+} 3 \mathrm{~d}^{2} \mathrm{G}_{9 / 2}$ metastable state) and the predicted slight decrease in $\mathrm{Ar}^{+}$density (which implies a $\sim 15 \%$ decrease in the $\operatorname{Ar}^{+} 3 \mathrm{~d}$ ${ }^{2} \mathrm{G}_{9 / 2}$ metastable state production rate), the $\mathrm{Ar}^{+}$LIF signal for a helium fraction of $27 \%$ is less than half $(\sim 43 \%)$ of the $\mathrm{Ar}^{+}$LIF signal for a helium fraction of $0 \% \mathrm{He}$ (see Figure $5.30(\mathrm{a}))$.

Because neutral helium has a number of high-energy metastable levels, e.g., $2 \mathrm{~s}$ ${ }^{3} \mathrm{~S}_{1}$ at $19.82 \mathrm{eV}$ and $2 \mathrm{~s}{ }^{1} \mathrm{~S}_{0}$ at $20.61 \mathrm{eV}$, other channels may contribute to populating the 
$\overline{\mathrm{Ar}^{+} 3 \mathrm{~d}^{2} \mathrm{G}_{9 / 2} \text { state. The first such mechanism considered is Penning ionization from } \mathrm{Ar}}$ ground state,

$$
\mathrm{He}^{*}\left(2 \mathrm{~s}^{3} \mathrm{~S}_{1}, 2 \mathrm{~s}{ }^{1} \mathrm{~S}_{0}\right)+\operatorname{Ar}\left(3 \mathrm{p}^{6}{ }^{1} \mathrm{~S}_{0}\right) \rightarrow \mathrm{He}\left(1 \mathrm{~s}^{2}{ }^{1} \mathrm{~S}_{0}\right)+\operatorname{Ar}^{+}\left(3 \mathrm{p}^{5}{ }^{2} \mathrm{P}_{3 / 2}^{0}\right)
$$

Although generally efficient, for our conditions this mechanism is unlikely to contribute significantly to the $\mathrm{Ar}^{+}$ground state population due to the small high-energy electron population; for helium fractions up to $30 \%$ the eedf is clamped by argon which impedes helium excitation to higher energy levels. Furthermore, elastic collisions with helium cool the eedf and reduce the population of helium metastable levels, thereby decreasing the efficiency of Penning ionization mechanism. A second mechanism that might increase the $\mathrm{Ar}^{+}$metastable population is the energy transfer process

$$
\operatorname{He}^{*}\left(2 \mathrm{~s}^{3} \mathrm{~S}_{1}, 2 \mathrm{~s}^{1} \mathrm{~S}_{0}\right)+\operatorname{Ar}^{+}\left(3 \mathrm{p}^{5}{ }^{2} \mathrm{P}^{0}{ }_{3 / 2}\right) \rightarrow \mathrm{He}\left(1 \mathrm{~s}^{2}{ }^{1} \mathrm{~S}_{0}\right)+\operatorname{Ar}^{+}\left(3 \mathrm{p}^{4} 3 \mathrm{~d}^{2} \mathrm{G}_{9 / 2}\right) \text {. }
$$

In spite of energy deficits for these reactions of only 0.7 and $1.5 \mathrm{eV}$ (the $\mathrm{Ar}^{+}$metastable level is $19.11 \mathrm{eV}$ above ion ground level), the same depleted high-energy tail of the eedf also makes this mechanism unlikely to play any role in $\mathrm{Ar}^{+}$metastable population. The decrease of the $\mathrm{Ar}^{+}$LIF signal could also be explained by a larger quenching crosssection of the $\mathrm{Ar}^{+}$metastable state due to collisions with ground state helium. Based on the calculated $\mathrm{Ar}^{+}$and helium relative populations and the discrepancy between the predicted $\mathrm{Ar}^{+}$population and LIF signal intensity, the $\mathrm{Ar}^{+}-\mathrm{He}$ quenching cross-section would have to be a factor of $\sim 6$ larger than the tabulated value for the $\mathrm{Ar}^{+}-\mathrm{Ar}$ quenching cross-section, $\sim 7 \times 10^{-14} \mathrm{~cm}^{2}$.

Another possibility involves charge-exchange collisions that create slow ions at the expense of hot ions. At zero or low helium fraction a deconvolution of the ivdf with two Gaussian distributions is straightforward and provides the flow speeds of the fast and slow ion groups (5.3 and $2.2 \mathrm{~km} / \mathrm{s}$ for the example shown in Figure 5.31 (a)). As the helium fraction increases, the fast group ivdf shifts toward higher speeds and a long tail towards slower speeds replaces the easily-defined slow ion group distribution. We note that similar Gaussian with long tail LIF profiles were reported in LIF ivdf observations of ions accelerated in an electrostatic presheath. ${ }^{51}$ Therefore, the tail of the fast ion ivdf is most probably a symptom of a drifting distribution slowed down by elastic scattering and/or charge exchange collisions with the background gas. Assuming an $\mathrm{Ar}_{-} \mathrm{Ar}^{+}$chargeexchange cross section $\sigma_{\mathrm{CX}}=4.7 \times 10^{-15} \mathrm{~cm}^{2}$ for the measured ion energies gives a $\mathrm{mfp}$ of 
$\sim 5 \mathrm{~cm}$ for pure argon plasma. For decreasing argon fraction, the mfp increases. It then follows that the longer tails (as can be seen in Figure 5.31 (b)) are the result of the EDL location moving a couple of centimeters deeper in the source (as a result of the longer $\mathrm{mfps}$ ). Fitting the distribution with only one Gaussian distribution corresponding to the fast component, integrating and then subtracting from the integral of the whole distribution, the ratio of the slow to fast ion populations can be calculated. To within errors of $\sim 10 \%$, the slow/fast ion population ratio is insensitive to the variations of the gas composition (see the scatter graph in Figure 5.30 (a)). Except the first and the last measurement values at helium fractions of $0 \%$ and $30 \%$, the slow ion population density is roughly $60 \%$ of the fast ion population density (dashed horizontal line). This observation is consistent with an increasingly efficient, non-velocity dependent quenching mechanism. In other words, as the helium fraction increases, the slow and fast $\mathrm{Ar}^{+}$metastable populations decrease at the same rate.
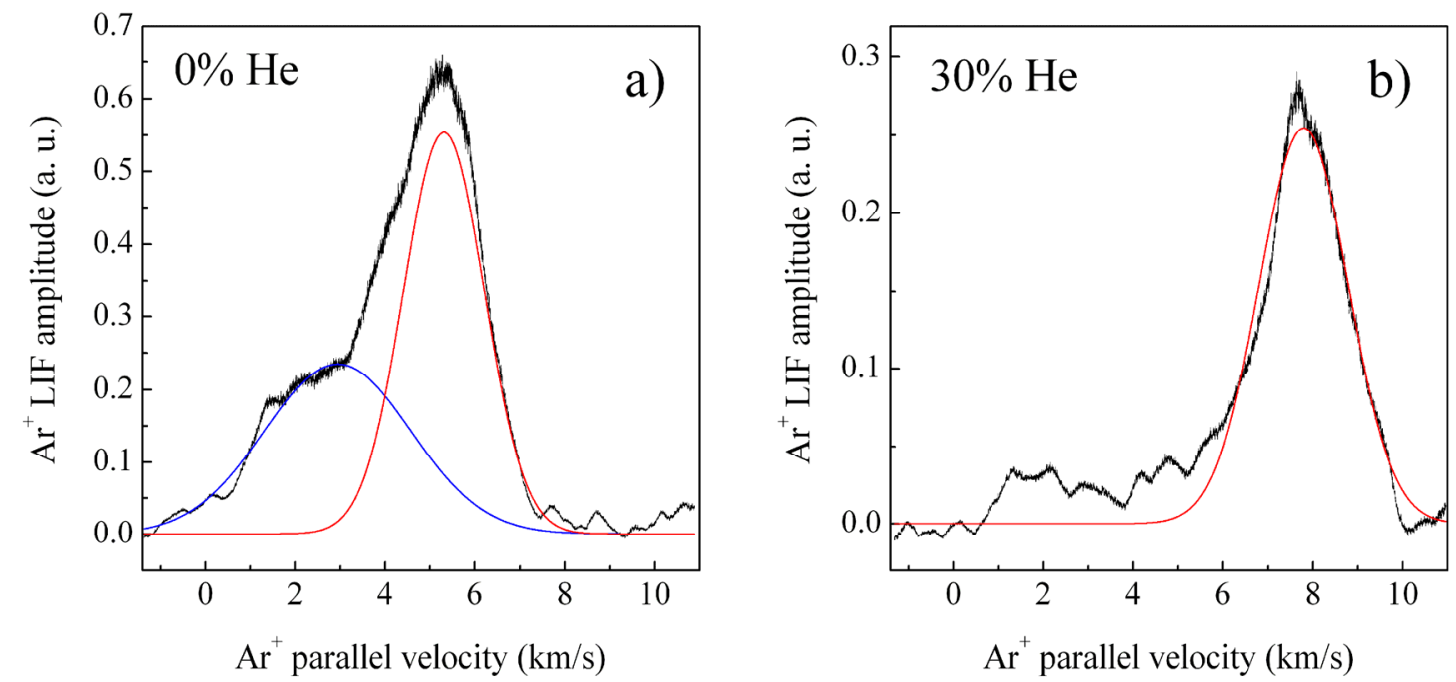

Figure 5.31. Examples of $\mathrm{Ar}^{+}$bimodal parallel ivdfs: a) for a $0 \%$ helium fraction the distribution is deconvolved into fast (red line) and slow (blue line) Gaussian distributions; b) for a 30\% helium fraction the distribution exhibits a long tail characteristic of charge-exchange collisions.

Perhaps the most interesting result of these investigations is the shift toward higher energies of the fast $\mathrm{Ar}^{+}$component ivdf as the helium fraction increases. As shown in Figures 5.30 (b) and 5.31, increasing the helium fraction from 0 to $30 \%$ increases the parallel $\mathrm{Ar}^{+}$flow speed from 5.3 to $7.8 \mathrm{~km} / \mathrm{s}$. In terms of kinetic energy, the fast $\mathrm{Ar}^{+}$ energy increases from $5.8 \mathrm{eV}$ to $12.6 \mathrm{eV}$. As already discussed, charge-exchange and 
Chapter 5: Electron and Ion Energy Distribution Functions in Two-Ion Species, Helicon Plasmas

Penning processes affect the $\mathrm{Ar}^{+}$metastable population or slow down the entire $\mathrm{Ar}^{+}$ distribution, but do not increase the EDL strength.

One possible explanation involves the decrease in the total pressure as the helium fraction increases. Sun et al. [42] found an empirical relationship for the parallel flow speed in pure argon plasmas,

$$
v_{\|}=B+A / p,
$$

where $A, B$ are free parameters and $p$ is the neutral pressure. From a simple model based on the momentum balance equation, they found that the EDL strength, i.e., the potential drop across the layer, increases with decreasing the neutral pressure. Since up to helium fractions of $30 \%$ the dominant ion is $\mathrm{Ar}^{+}$(see Figure 5.28 (b)), Eq. (5.11) should hold for Ar-He plasmas. That this model provides an accurate prediction for the $\mathrm{Ar}^{+}$flow speed is demonstrated in Figure 5.30 (b), where the experimentally determined flow velocities and the equivalent expression

$$
v_{\|}=\alpha+\beta /(1-\gamma x),
$$

where $\alpha, \beta, \gamma$ are free parameters and $x$ is the helium fraction are shown on the same plot. One significant difference between these mixed gas experiments and the pure argon plasma results was that in pure argon plasma, the EDL strength dependence on pressure was equivalent to a dependence on the electron temperature. In these mixed $\mathrm{Ar}-\mathrm{He}$ plasmas (up to helium fractions of $30 \%$ ), the electron temperature does not vary, yet the ion parallel kinetic energy still doubles. 


\section{Chapter 5 References}

${ }^{1}$ I. D. Sudit and R. C. Woods, Rev. Sci. Instrum. 64, 2440 (1993)

2 J. E. Chilton, J. B. Boffard, R. S. Schappe, and C. C. Lin, Phys. Rev. A 57, 267 (1998)

${ }^{3}$ J. B. Boffard, C. C. Lin, and C. A. DeJoseph, J. Phys. D: Appl. Phys. 37, R143 (2004)

${ }^{4}$ V. M. Donnelly, J. Phys. D: Appl. Phys. 37, R217 (2004)

${ }^{5}$ J. B. Boffard, B. Ciaro, T. Weber and C. C. Lin, Atomic Data and Nuclear Data Tables 93, 831 (2007)

${ }^{6}$ Y. Chiu, B. L. Austin, S. Williams, and R. A. Dressler, J. Appl. Phys. 99, 113304 (2006)

${ }^{7}$ J. T. Fons and C. C. Lin, Phys. Rev. A 58, 4603 (1998)

${ }^{8}$ T. Czerwiec and D. B. Graves, J. Phys. D: Appl. Phys. 37, 2827 (2004)

${ }^{9}$ G. D. Severn, D. A. Edrich and R. McWilliams, Rev. Sci. Instrum. 69, 10 (1998)

${ }^{10}$ L. Broström, S. Mannervik, A. Passian and G. Sundström, Phys. Rev. A 49, 3333 (1994)

${ }^{11}$ A. I. Strinić, G. N. Malović, Z. Lj. Petrović and N. Sadeghi, Plasma Sources Sci. Technol. 13, 333 (2004)

${ }^{12}$ A. M. Keesee and E. E. Scime, Plasma Sources Sci. Technol. 16, 742 (2007)

${ }^{13}$ J. Boffard, J. Phys. D: Appl. Phys. 37, R143 (2004)

${ }^{14}$ C. Charles, Plasma Sources Sci. Technol. 16, R1 (2007)

${ }^{15}$ C. Biloiu, X. Sun, E. Choueiri, F. Doss, E. Scime, J. Heard, R. Spektor, and D. Ventura, Plasma Sources Sci. Technol. 14, 359 (2005)

${ }^{16}$ A. M. Keesee, E. E. Scime, C. Charles, and R. Boswell, Phys. Plasmas 12, 093502 (2005)

${ }^{17}$ C. Charles and R. W. Boswell, Appl. Phys. Lett. 91, 201505 (2007)

${ }^{18}$ Z. Harvey, S. Chakraborty Thakur, A. Hansen, R. Hardin, W. S. Przybysz, and E. E. Scime, Rev. Sci. Instrum. 79, 10F314 (2008)

${ }^{19}$ S. A. Cohen, N. S. Siefert, S. Stange, R. F. Boivin, E. E. Scime, and F. M. Levinton, Phys. Plasmas 10, $2593(2003)$

${ }^{20}$ X. Sun, S. A. Cohen, E. E. Scime, and M. Miah, Phys. Plasmas 12, 103509 (2005)

${ }^{21}$ E. Scime, R. Hardin, C. Biloiu, A. M. Keesee, and X. Sun, Phys. Plasmas 14, 043505 (2007)

${ }^{22}$ W. M. Manheimer and R. F. Fernsler, IEEE Trans. Plasma Sci. 29, 75 (2001)

${ }^{23}$ C. S. Corr, J. Zanger, R. W. Boswell, and C. Charles, Appl. Phys.Lett. 91, 241501 (2007)

${ }^{24}$ C. Charles and R. W.Boswell, Appl. Phys.Lett. 82, 1356 (2003)

${ }^{25}$ X Sun, A. M. Keesee, C. Biloiu, E. E. Scime, A. Meige, C. Charles, and R. W. Boswell, Phys. Rev.Lett. 95, 025004 (2005)

${ }^{26}$ C. Charles, Appl. Phys.Lett. 84, 332 (2004)

${ }^{27}$ C. Charles, R. W.Boswell and M. A. Lieberman, Appl. Phys.Lett. 89, 261503 (2006)

${ }^{28}$ C. Charles, R. W.Boswell, R. Laine, and P. MacLellan, J. Phys. D: Appl. Phys. 41, 175213 (2008)

${ }^{29}$ K. P. Shamrai, V. F. Virko, Y. V. Virko, and K. Toki, Mini-conference on energetic ions and electrons in helicon sources - APS-DPP Conference, Orlando (2007) 


\section{Chapter 5: Electron and Ion Energy Distribution Functions in Two-Ion Species, Helicon Plasmas}

${ }^{30}$ J. L. Kline, E. E. Scime, R. F. Boivin, A. M. Keesee, and X. Sun, Plasma Sources Sci. Technol. 11, 413 (2002)

${ }^{31}$ H. Salami and A. Ross, J. Molec. Spectr. 233, 157 (2005)

${ }^{32} \mathrm{http}: / /$ physics.nist.gov/PhysRefdData/ASD?index.html

${ }^{33}$ CRC Handbook of Chemistry and Physics 68-th edition, Edts R. C. Weast, M. J. Astle, W. H. Beyer, CRC Press Inc, Boca Raton, FL 1988

${ }^{34}$ C. J. Humphrey, J. Res. Nat. Bur. Stand. 23, 683 (1939)

${ }^{35}$ C. J. Humphrey, J.Opt. Soc. Am. 60, 1454 (1970)

${ }^{36}$ J. E. Hansen and W. Persson, Phys. Scripta 36, 602 (1987)

37 N. Sadeghi, N. Dorval, J. Bonnet, D. Pigache, C. Kadlec-Philippe, and A. Bouchoule, 35-th AIAA/ASM/SAE/ASEE/ Joint Propulsion Conference \& Exhibit, June 20-23, Los Angeles, CA (1999)

${ }^{38}$ R. J. Cedolin, W. A. Hargus Jr., P. V. Storm, R. K. Hanson, and M. A. Cappelli, Appl. Phys. B 65, 459 (1997)

${ }^{39}$ R. J. Cedolin, PhD Dissertation, Stanford University (1997)

${ }^{40}$ B. Krames, Th. Glenewinkel-Meyer, and J. Meichsner, J. Appl. Phys. 89, 3115 (2001)

${ }^{41}$ R. F. Boivin, West Virginia University Plasma Physics Lab Report No. PLP-046 (2000)

${ }^{42}$ X Sun, C. Biloiu, R. Hardin, and E. E. Scime, Plasma Sources Sci. Technol. 13, 359 (2004)

${ }^{43}$ N. Y. Babaeva, J. K. Lee, and J. W. Shon, J. Phys. D: Appl. Phys. 38, 287 (2005)

${ }^{44}$ N. Sadeghi, T. Nakano, D. J. Trevor, and R. A. Gottscho, J. Appl. Phys. 70, 2552 (1991)

${ }^{45}$ G. Gozadinos, D. Vender, and M. M. Turner, unpublished presentation (2002)

${ }^{46}$ A. V. Phelps, J. Appl. Phys. 76, 747 (1994)

${ }^{47}$ J. S. Miller, S. H. Pullins, D. J. Levandier, Y. Chiu, and R. A. Dressler, J. Appl. Phys. 91, 984 (2002)

${ }^{48}$ M. Lieberman and A. Lichtenberg, Principles of Plasma Discharges and Material Processing, John Wiley \& Sons, New York (1994)

${ }^{49}$ A. W. Degeling, T. E. Sheridan, and R. W. Boswell, Phys. Plasmas 6, 3664 (1999)

${ }^{50}$ V. Kaeppelin, M.Carrere, and J. B. Faure, Rev. Sci. Instrum. 72, 4377 (2001)

${ }^{51}$ N. Claire, G. Bachet, U. Stroth and F. Doveil, Phys. Plasmas 13, 062103 (2006) 


\section{Chapter 6: Temporal Evolution of the Parallel IVDF in Pulsed Argon Plasma}

\subsection{Time-Resolved Laser Induced Fluorescence}

In Chapter 4.3.2, the principles of time-resolved laser induced fluorescence were reviewed. The experiments presented here concern the application of time-resolved LIF to pulsed helicon plasma. The experimental configuration for time resolved LIF is shown in Figure 4.21. The $5 \mathrm{~Hz}$ pulsed operation of the helicon source plasma was accomplished by amplitude modulation of the $9.5 \mathrm{MHz}$ driving frequency. $750 \mathrm{~W}$ of rf power was matched through an $m=+1$ helical antenna to the helicon source filled with flowing argon at $20 \mathrm{sccm}$ and at an operating pressure of $2.5 \mathrm{mTorr}$. The magnetic field strength on the axis was $700 \mathrm{G}$. For these operating conditions, but in steady-state mode, typical plasma parameters, as measured with an rf compensated Langmuir probe, ${ }^{1}$ are an electron temperature of $\approx 7 \mathrm{eV}$ and an electron density of $\approx 1.2 \times 10^{12} \mathrm{~cm}^{-3}$. The time-resolved LIF measurements were obtained at $z=146 \mathrm{~cm}$ in HELIX, i.e. $4 \mathrm{~cm}$ upstream of the HELIXLEIA junction. As shown in Figure 6.1 (a), for integration times of the lock-in amplifier between 0.1 and $1 \mathrm{~ms}$, the LIF signal increases nearly linearly with the logarithm of the chopping frequency. There is a threshold value of the integration time, $\sim 100 \mu \mathrm{sec}$ (see Figure 6.1 (b)), below which the detected LIF signal drops abruptly. For this detection scheme, the minimum integration time at which LIF signal could be detected was 30 $\mu \mathrm{sec}$. For an integration time of $30 \mu \mathrm{sec}$, the LIF signal is about $60 \%$ of the value obtained with a $1 \mathrm{~ms}$ integration time. There is a trade-off between the chopping frequency and the transmitted laser power: higher chopping frequency can be achieved with the acousto-optic modulator (AOM), but the transmitted laser power is significantly lower than when the mechanical chopper is employed. For integration times shorter than $30 \mu \mathrm{sec}$, the lock-in amplifier was unable to discriminate between the induced fluorescence emission and the plasma spontaneous emission. A $30 \mu \mathrm{sec}$ integration time does not necessarily yield a temporal resolution of $30 \mu \mathrm{sec}$. Another factor limiting the temporal resolution is the digitization rate of the oscilloscope. LIF signal recording, 
averaging, and digitization was accomplished with a Tektronix TDS 460A oscilloscope at a digitization rate of $10 \mathrm{kHz}$, thereby limiting the time resolution of the method to 100 $\mu$ sec.
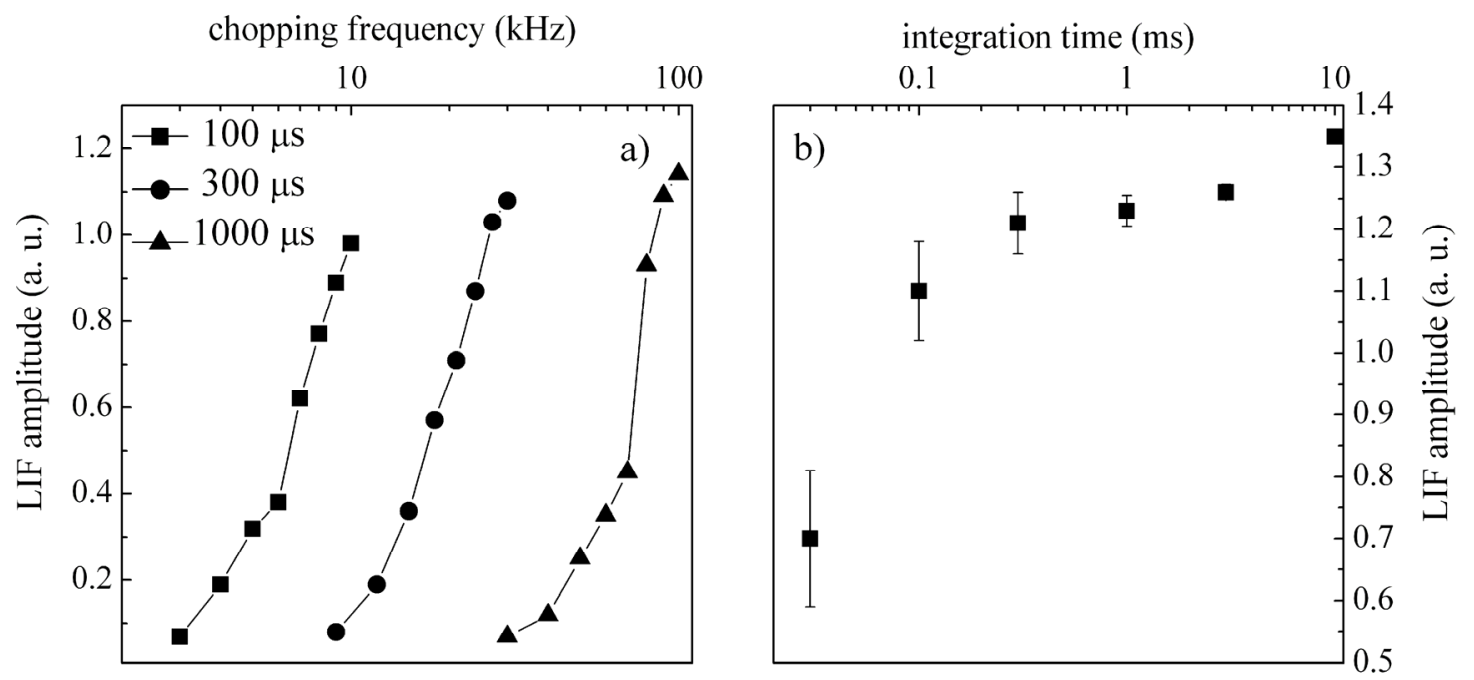

Figure 6.1. a) $\mathrm{Ar}^{+}$LIF signal amplitude versus chopping frequency for three different integration times and b) versus integration time for a $100 \mathrm{kHz}$ chopping frequency.

For a reliable ivdf reconstruction, typically 25 equally spaced laser wavelengths centered on the wavelength corresponding to the peak LIF signal were used. Adequate signal-tonoise levels were obtained when the measurements at each wavelength were averaged over 300 plasma pulses. For our low temperature plasma, a wavelength span of $0.012 \mathrm{~nm}$ is sufficient to measure the entire ivdf for argon ions with a $0.4 \mathrm{eV}$ temperature.

Increased fluorescence as the laser wavelength is tuned through the absorption line is evident in Figure 6.2 (a). Although noisy, LIF signal appears throughout the pulse except at the very beginning. During the first approximately $26 \mathrm{~ms}$ of the pulse, the LIF signal oscillates with a characteristic frequency of about $1 \mathrm{kHz}$ (see Figure 6.2 (b)). The oscillations are observed at all laser wavelengths, are unaffected by the rf amplitude modulation frequency, and vanish at long integration times. They cannot be electronic noise pickup because they vanish if plasma light entering the collection optics is blocked. It is expected that any naturally occurring fluctuations in the plasma with frequencies on the order of $1 \mathrm{kHz}$ would be rejected by the lock-in detection scheme, i.e., on the 100 $\mathrm{kHz}$ modulation timescale of the AOM the background light signal fluctuations at $1 \mathrm{kHz}$ 
would be essentially constant and therefore result in a net null signal. However, if the oscillations result from a large and decaying initial oscillation (as in a damped oscillator), the change in oscillation amplitude as a function of time could result in a finite signal even with the lock-in detection scheme.

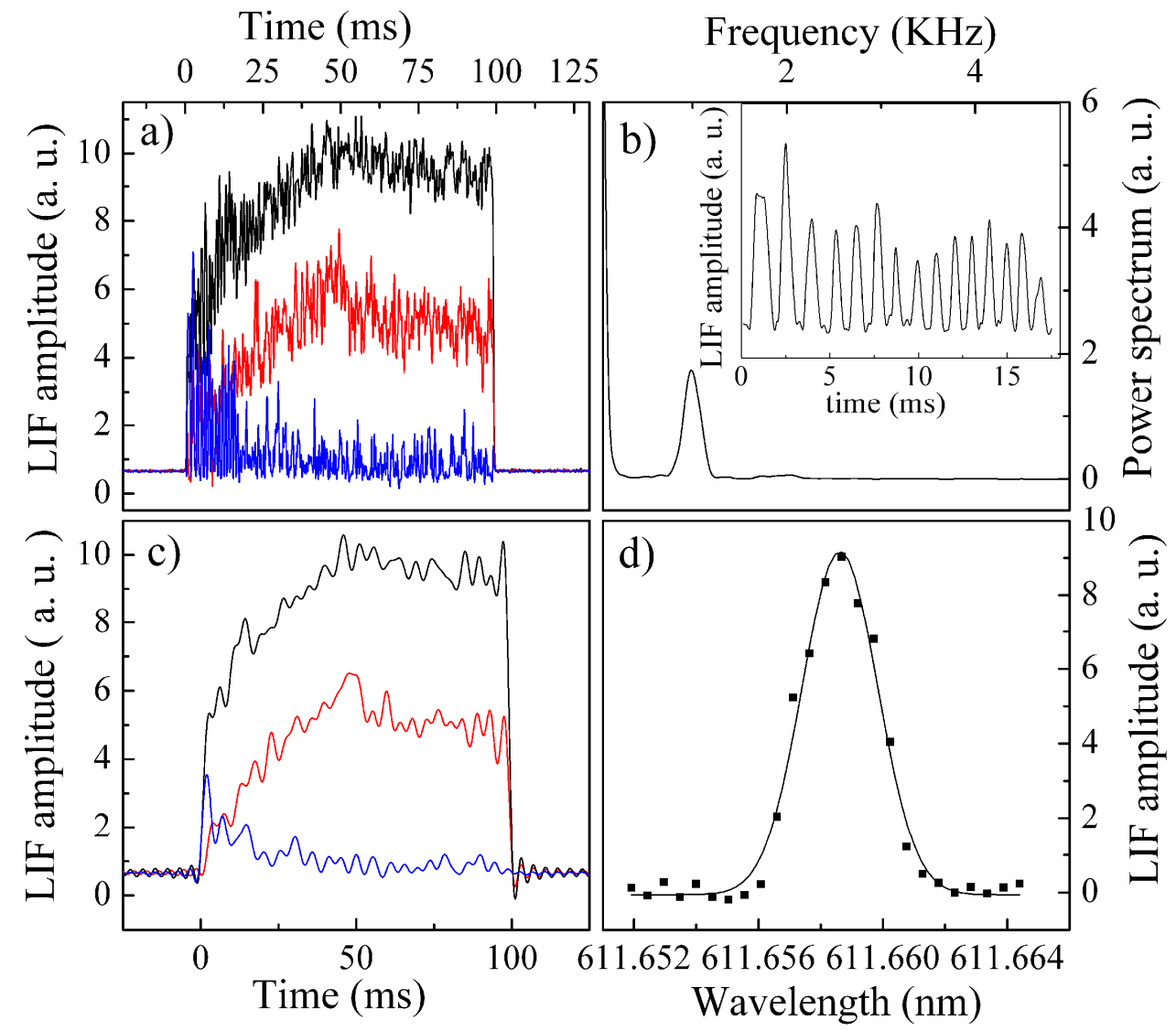

Figure 6.2. a) Raw LIF signal during the $100 \mathrm{~ms}$ discharge pulse (only 3 of 25 wavelengths are plotted); b) Oscillations in the LIF signal amplitude observed on the first $26 \mathrm{~ms}$ of the pulse (as inset) and corresponding power spectrum showing $1 \mathrm{kHz}$ oscillation frequency; c) LIF signal after low pass filtering; d) Argon ivdf at $t=50 \mathrm{~ms}$ into the pulse.

Such an interpretation is consistent with the vanishing of the $1 \mathrm{kHz}$ signal later in the discharge pulse, i.e., when the oscillation amplitude becomes more constant in time. To eliminate the $1 \mathrm{kHz}$ oscillation, the raw data were digitally low pass filtered. The processed signals are shown in Figure 6.2 (c). A typical ivdf (at $t=50 \mathrm{~ms}$ into the discharge pulse) is shown in Figure 6.2 (d). The experimental values are well fit by a single Maxwellian distribution, indicating that no EDL upstream of observation point is present for these plasma operating conditions. 

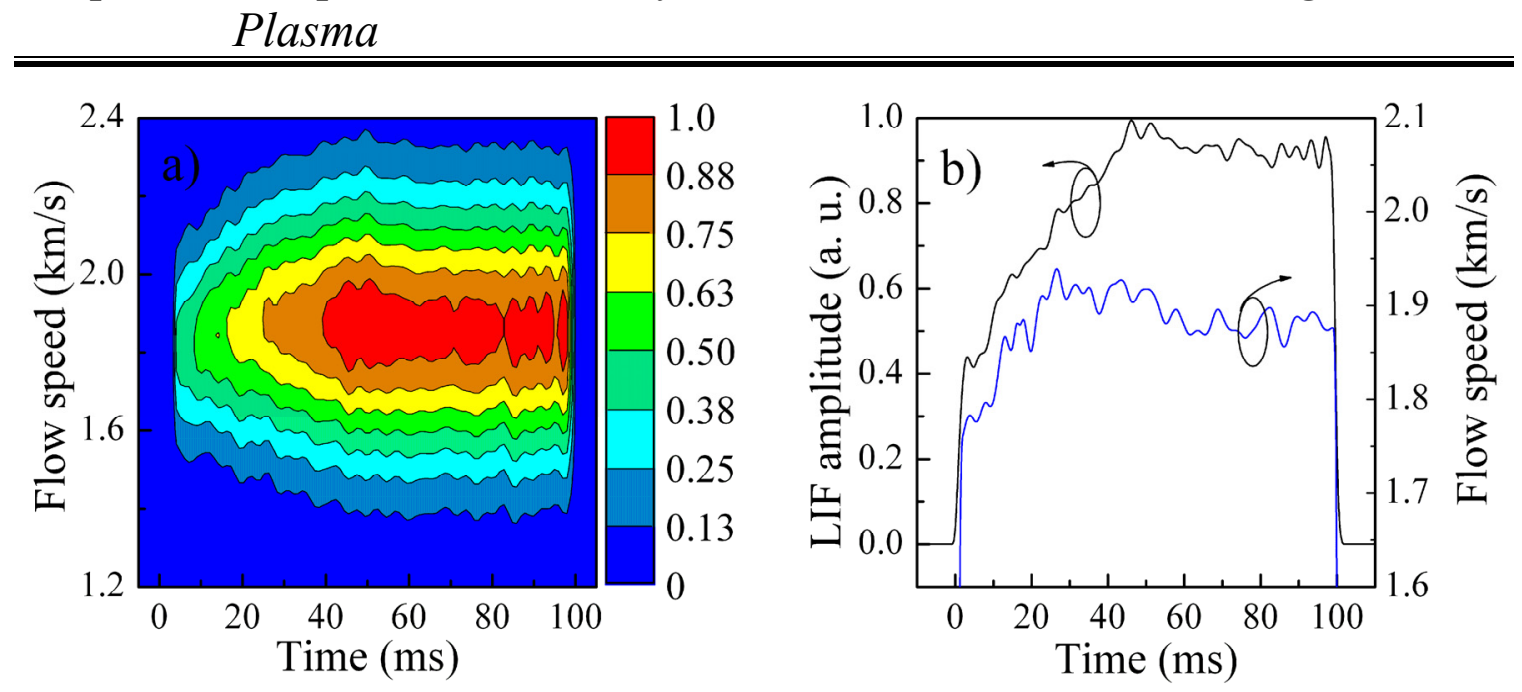

Figure 6.3. a) Contour plot of the evolution of the argon ion vdf during the $100 \mathrm{~ms}$ pulse with $100 \mu \mathrm{s}$ time resolution; b) Evolution of the LIF signal amplitude (black line) and ion flow velocity (blue line) during the pulse

The time evolution of the argon ivdf in the absence of EDL is shown in Figure 6.3 (a) with $100 \mu \mathrm{sec}$ time resolution. These high time resolution measurements revealed features of the argon ivdf that were not observed in $1 \mathrm{~ms}$ time resolution measurements: ${ }^{2}$ the signal amplitude and ion temperature increase slowly during the pulse and require approximately $45 \mathrm{~ms}$ to reach their steady state values (see Figure 6.3 (b) ); the ion flow speed reaches its final value of $\sim 1.9 \mathrm{~km} / \mathrm{s}$ much more quickly (after approximately 25 $\mathrm{ms}$ ); and an average ion flow of over $1.5 \mathrm{~km} / \mathrm{s}$ appears within the first few hundred $\mu \mathrm{sec}$ into the discharge. Because the LIF signal is roughly proportional to the ion density, the $45 \mathrm{~ms}$ timescale to achieve steady state LIF amplitude and ion temperature likely reflects the time necessary for the discharge to completely break down and reach a steady state ion density as well as to heat the ions from room temperature to $0.4 \mathrm{eV}$. The more rapid ion acceleration suggests that the time scale needed to create the electric fields responsible for ion acceleration (discussed in Ref. [3]) is shorter and distinct from the overall discharge evolution.

The time-resolved LIF technique described here has achieved high quality measurements at $100 \mu \mathrm{s}$ with only minor modifications to a standard LIF diagnostic. Similar quality data at a time resolution of $30 \mu$ s is possible with slightly improved light collection and a faster oscilloscope. At lower time resolution, the large oscillations in optical emission from the plasma had gone undetected. We note that the oscillation 
amplitude vanishes at approximately the same time that the ion acceleration ceases. The similarity in time scales suggests that the two phenomena are related and further investigation is warranted.

\subsection{Temporal Evolution of Bimodal Argon IVDF in Expanding Helicon Plasma}

Spontaneously appearing ion beams in the diverging magnetic field region downstream of low pressure helicon sources (believed to result from the formation of a current-free EDL) might provide an alternative to classical chemical propulsion for spacecraft. ${ }^{4,5}$ In a thruster application the ejected plasma flux and the ion exit velocity are the critical parameters. As shown in the previous chapter, promising levels of ion production $\left(\sim 10^{13} \mathrm{~cm}^{-3}\right.$ plasma density) by helicon sources and supersonic ion exit speeds (between 8 and $15 \mathrm{~km} / \mathrm{s}$ ), ${ }^{6}$ suggest the possibility of a helicon source EDL thruster. ${ }^{7,8}$ Pulsing the helicon discharge might solve some important thruster issues such as plasma detachment, turbulent cross-field diffusion, and antenna heating. Therefore, for thruster applications, an understanding of the temporal evolution of the ion velocity distribution function (ivdf) downstream of an expanding helicon source is needed to choose the optimal operational parameters (duty cycle, pulse length, input power, driving frequency, etc.) to obtain the desired specific impulse along the expansion direction while minimizing the ion energy in the perpendicular direction. Two diagnostic techniques have the capability of investigating the temporal behavior of the ivdf in pulsed plasmas: a) a time resolved retarding field energy analyzer (RFEA) ${ }^{9}$ and b) time resolved laser induced fluorescence (LIF). ${ }^{10,11}$ Each method has its own advantages and disadvantages. The RFEA method is perturbative and requires conversion of energy-space measurements into ivdf measurements - a process very sensitive to the effects of the sheath created in the front of the grounded RFEA probe. ${ }^{12}$ LIF directly measures the ivdf without perturbation. However, the RFEA method provides information on the entire ion population while the LIF measured ivdf only reflects the behavior of the population of a particular excited ion state (for the 3 level LIF scheme $3 \mathrm{~d}^{2} \mathrm{G}_{9 / 2} \rightarrow 4 \mathrm{p}^{2} \mathrm{~F}_{7 / 2} \rightarrow 4 \mathrm{~s}^{2} \mathrm{D}_{5 / 2}$ usually employed for argon ion investigation, it is the metastable state $3 \mathrm{~d}^{2} \mathrm{G}_{9 / 2}$ ). However, as we have shown for the 
continuous wave LIF, with an adequate model the LIF signal intensity for this particular scheme is a qualitative measure of the behavior of the entire ion population.

These experiments were performed on the HELIX-LEIA system. The rf power necessary to drive the helicon plasma source was pulsed by square wave amplitude modulation ( $5 \mathrm{~Hz}$ at a $50 \%$ duty cycle) of the $9.5 \mathrm{MHz}$ driving frequency. For $\mathrm{Ar}^{+} \mathrm{LIF}$, we used the classic three-level LIF scheme described previously. To determine the parallel ivdf, laser light was injected along the axis of the source and the fluorescence signal detected by an integrated collection optics-photomultiplier tube mounted on the previously described scanning probe. Temporal resolution of $1 \mathrm{~ms}$ was achieved by modulation of the laser beam with an acousto-optic modulator at $10 \mathrm{kHz}$. The in-phase and out-of-phase lock-in amplifier signals were recorded with a digital oscilloscope synchronized to the rf modulation signal. Very good signal-to-noise $(\mathrm{S} / \mathrm{N})$ was obtained when the LIF signals were averaged over 400 plasma pulses. Because the plasma conditions were chosen to result in EDL formation, the detected ivdf was bimodal. To obtain a reliable reconstruction of the ivdf, the plasma was interrogated at 71 equally spaced laser wavelengths. After subtraction of the time-dependent background, the S/N ratio was better than 10:1.

The evolution of the parallel argon ivdf at $z=169 \mathrm{~cm}(19 \mathrm{~cm}$ downstream helicon source-diffusion chamber junction) is shown in Figure 6.4. Since previous investigations showed oscillations of the LIF signal with a characteristic frequency of $1 \mathrm{kHz}^{13}$ when the lower hybrid frequency in the source was comparable to the rf frequency, the source was operated at magnetic fields of $600 \mathrm{G}$ in the source and $35 \mathrm{G}$ in the diffusion chamber. Consistent with the continuous wave operation observations, the ivdf exhibits a bimodal structure: a fast population with flow speeds of $\sim 7.1 \mathrm{~km} / \mathrm{s}$ and a slow population with speeds of $\sim 0.4 \mathrm{~km} / \mathrm{s}$. For similar steady-state plasma parameters, probe measurements indicated a potential drop of $\sim 18 \mathrm{~V}$, corresponding to an EDL strength of $\sim 3 k_{B} T_{e} / e-$ sufficient to accelerate the fast ions to the measured speeds. The slow ion group LIF intensity is higher at the beginning of the pulse and persists for a few ms after the rf pulse terminates. The LIF signal for the fast ion population disappears at the end of the rf pulse, most likely an effect of rapid quenching of the $3 \mathrm{~d}^{2} \mathrm{G}_{9 / 2}$ metastable state as these ions travel from HELIX into LEIA. The most significant feature in the measurements is the 
228 ms time lag in the appearance of the fast ion population..$^{14}$ This observation does not necessarily contradict previous RFEA measurements in another helicon source that indicated the presence of a small, but finite, population of fast ion population from the very beginning of the rf pulse [9]. RFEA measurements are essentially current measurements and are therefore particularly sensitive to fast ions. Secondly, this effect could be specific to our experiment given the differences between HELIX-LEIA and ChiKung helicon machines.
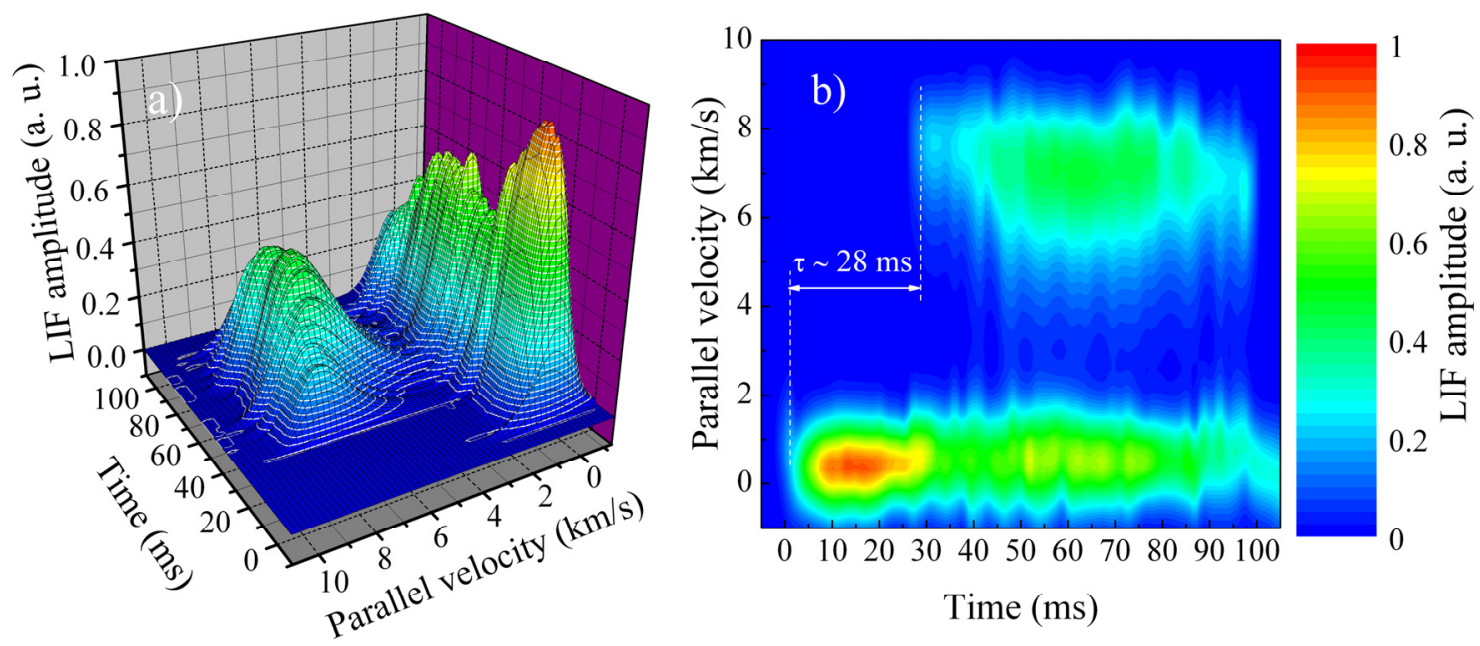

Figure 6.4. With $1 \mathrm{~ms}$ time resolution, the LIF-determined argon ion velocity distribution function during a 100 ms plasma pulse: a) surface plot showing fast $(\sim 7.1 \mathrm{~km} / \mathrm{s})$ and a slow $(\sim 0.4 \mathrm{~km} / \mathrm{s})$ ion populations; $\mathrm{b})$ contour plot showing the time lag $(\sim 28 \mathrm{~ms})$ in the appearance of the fast ion population.

To determine if the observed time lag for fast ion creation in the LIF data reflects the time necessary for the population of the $3 \mathrm{~d}^{2} \mathrm{G}_{9 / 2}$ level to become large enough for a measurable LIF signal, ivdfs were measured for different duty cycles and pulse frequencies. The evolution of the parallel argon ivdf for the same $5 \mathrm{~Hz}$ pulsed discharge but for $80 \%$ duty cycle is shown in Figure 6.5. The LIF-determined argon ivdf during a $160 \mathrm{~ms}$ "plasma on" pulse shows that the slow and fast ion population parallel velocities do not vary with "plasma on" time: $\sim 0.4 \mathrm{~km} / \mathrm{s}$ and $\sim 7 \mathrm{~km} / \mathrm{s}$ for the parallel flow speed of the slow and fast ion populations. However, increasing the "plasma on" time (160 ms versus the $100 \mathrm{~ms}$ in Figure 6.4) decreased to $\sim 7 \mathrm{~ms}$ the time delay in the appearance of the fast ion population. Long lived neutral and ionic metastable states can survive during "plasma off" time, making the plasma ignition and apparently ion beam formation faster for shorter time off intervals. However, since the LIF signal for the background ions 
appears at the onset of the discharge regardless of the length of the "plasma off" time, the time lag in the ion beam LIF signal is not a result time needed to create a population of appropriate ion metastable states.
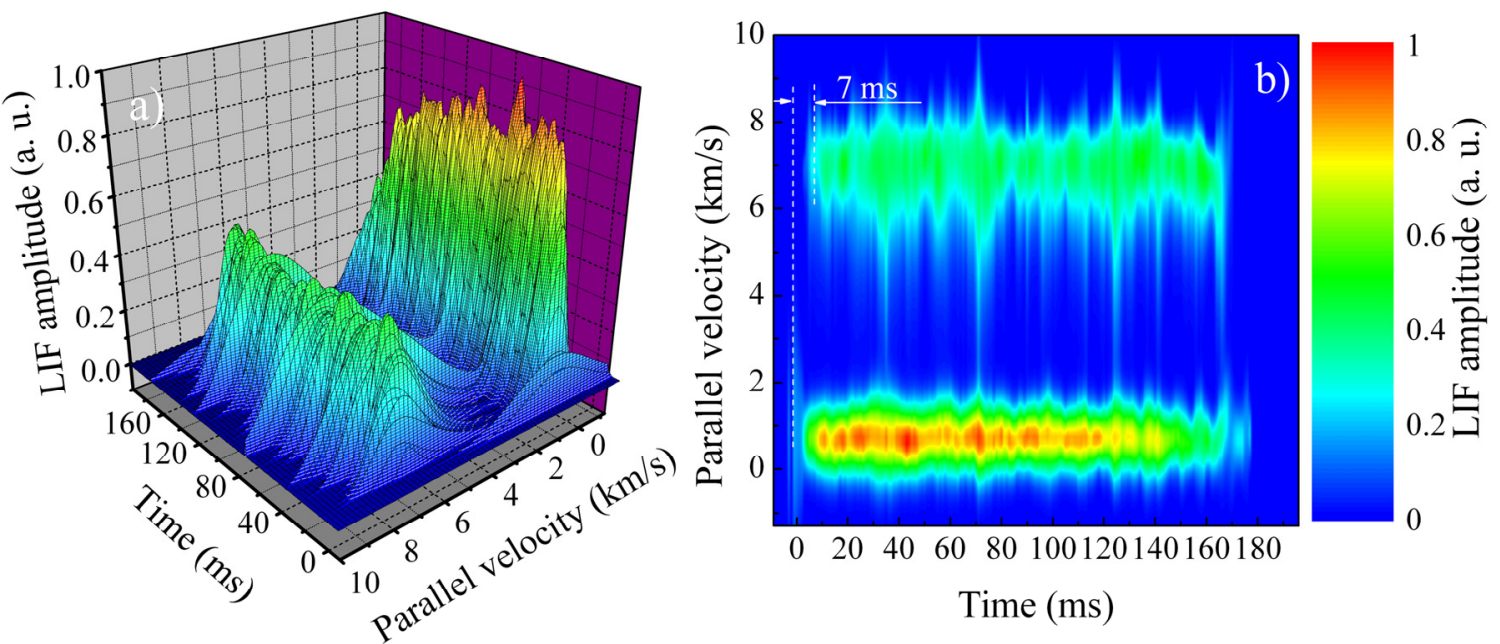

Figure 6.5. The evolution of argon ivdf during a $160 \mathrm{~ms}$ plasma pulse: a) surface plot showing fast $(\sim 7$ $\mathrm{km} / \mathrm{s})$ and a slow $(\sim 0.4 \mathrm{~km} / \mathrm{s})$ ion populations; b) contour plot showing the time lag $(\sim 7 \mathrm{~ms})$ in the appearance of the fast ion population.

Another time-resolved study of the ivdf was performed by fixing the duty cycle at $50 \%$ but increasing the rf power modulation frequency to $10 \mathrm{~Hz}$. Figure 6.6 shows the evolution of argon ivdf $19 \mathrm{~cm}$ downstream the HELIX-LEIA junction during the $50 \mathrm{~ms}$ "plasma on" time for this case. Compared to the results shown in Figure 6.4, the increase in pulsing frequency did not affect the parallel velocities of the slow and fast argon ions. However, the longer "plasma off" time resulted in an increase in the time lag in the appearance fast ion population to $19 \mathrm{~ms}$. The different behavior of the LIF intensity distribution during the pulse emphasize the different origins of the ions: the fast ion group appears later and disappears when the "plasma on" pulse terminates, whereas the slow ion group appears simultaneously with the plasma formation but extends into the afterglow. The variations in the LIF intensity of the background ion population during the pulses suggest that longer "plasma off" times lead to a complete extinguishing of the discharge between pulses followed by a new breakdown (high LIF intensities at the beginning of plasma pulse). 


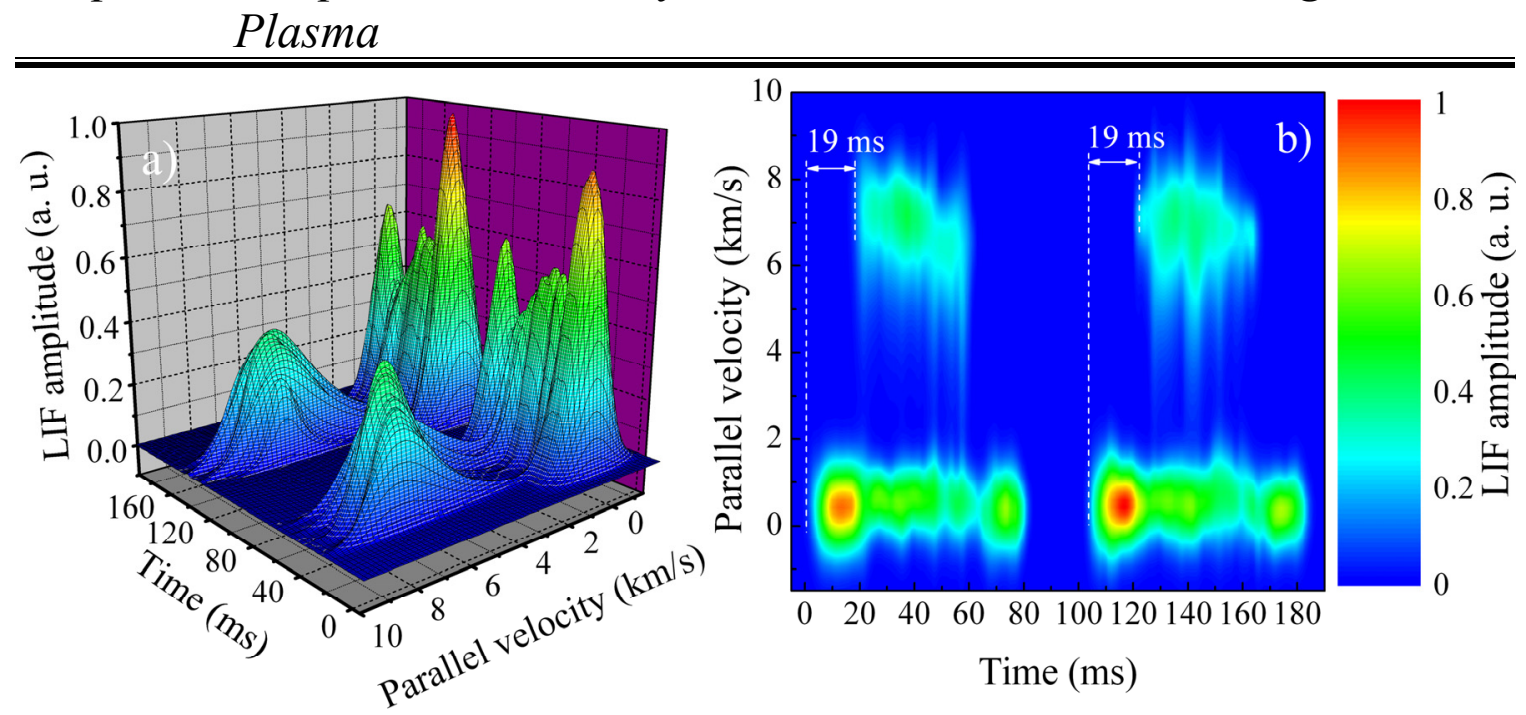

Figure 6.6. The evolution of the argon ivdf during a $50 \mathrm{~ms}$ plasma pulse: a) surface plot showing fast ( $7.1 \mathrm{~km} / \mathrm{s})$ and a slow $(\sim 0.3 \mathrm{~km} / \mathrm{s})$ ion populations; b) contour plot showing the time lag $(\sim 19 \mathrm{~ms})$ in the appearance of the fast ion population.

The major implication of these time-resolved LIF measurements is that the time delay for the appearance of the fast argon ion population downstream of the HELIXLEIA junction depends strongly on the pulse repetition frequency and the pulse duty cycle, being shorter for shorter off time period. This observation can be useful in designing thrusters. For example, to optimize the overall energetic budget, electron flood gun operation for space charge neutralization can be limited to the "plasma on" time. 


\section{Chapter 6 References}

${ }^{1}$ C. Biloiu, E. Scime, X. Sun, and B. McGeehan, Rev. Sci. Instrum. 75, 4296 (2004)

${ }^{2}$ E. Scime, C. Biloiu, C. Compton, F. Doss, D. Ventura, J. Heard, E. Choueri, and R. Spektor, Rev. Sci. Instrum. 76, 026107 (2005)

${ }^{3}$ X Sun, C. Biloiu, R. Hardin, and E. E. Scime, Plasma Sources Sci. Technol. 13, 359 (2004)

${ }^{4}$ C. Charles, Plasma Sources Sci. Technol. 16, R1 (2007)

${ }^{5}$ R. Walker, N. Plihon, P. Chabert, and J. L. Raimbault , 42-nd AIAA Joint Propulsion Conference 4844 (2006)

${ }^{6}$ X Sun, A. M. Keesee, C. Biloiu, E. E. Scime, A. Meige, C. Charles, and R. W. Boswell, Phys. Rev. Lett. 95, 025004 (2005)

${ }^{7}$ C. Charles and R. W. Boswell, Patent Application PS 3033/02 (2002)

${ }^{8}$ N. Plihon, P. Chabert, and J. L. Raimbault, European Spatial Agency Report ARI 04/3101 (2006)

${ }^{9}$ C. Charles and R. Boswell, Phys. Plasmas 11, 3808 (2004)

${ }^{10}$ G. Bachet, F. Skiff, F. Doveil, and R. A. Stern, Phys. Plasmas 8, 3535 (2001)

${ }^{11}$ C. Biloiu, X. Sun, E. Choueri, F. Doss, E. Scime, R. Spektor, J. Heard, and D. Ventura, Plasma Sources Sci. Technol. 14, (2005) 766

${ }^{12}$ Z. Harvey, S. Chakraborty Thakur, A. Hansen, R. Hardin, W. S. Przybysz, and E. E. Scime, Rev. Sci. Instrum. 79, 10F314 (2008)

${ }^{13}$ I. A. Biloiu, X. Sun and E. E. Scime, Rev. Sci. Instrum. 77, 10F301 (2006)

${ }^{14}$ I. A. Biloiu and E. E. Scime, IEEE Trans. Plasma Sci. 36, 1376 (2008) 


\section{Chapter 7: Oblique and Tomographic LIF Measurements of the EDL in Argon Plasma}

The development of techniques for analysis of LIF data obtained by laser injection at oblique angles relative to the direction of flow or to the magnetic field in magnetized plasmas is limited. ${ }^{1}$ Historically, researchers have gone to considerable effort to ensure that the laser light is injected either perfectly parallel or perfectly perpendicular to the ambient magnetic field, ${ }^{2,3}$ even when optical access is severely limited. ${ }^{4} \mathrm{We}$ describe analysis of LIF data obtained during oblique injection of the probe laser relative to the background magnetic field of a linear system. The analysis method is applied to one-and two- dimensional velocity space studies of a bimodal argon ion velocity distribution function (ivdf) obtained in the expansion region of a helicon plasma source. Previous investigations have shown that for source pressures less than 2 mTorr, an ion accelerating electric field, most likely an EDL, spontaneously forms at the end of an expanding helicon source plasma. ${ }^{5}$ For our helicon source - diffusion chamber (HELIXLEIA) system and at a LEIA magnetic field of approximately $70 \mathrm{G}$, the double layer appears a few centimeters inside the source where the axial magnetic field gradient is a maximum. ${ }^{6}$ The EDL alters the downstream ion velocity distribution from unimodal to bimodal; consisting of a supersonic ion population superimposed on a nearly stationary background ion population. For these argon plasma experiments, we used the same $\mathrm{Ar}^{+}$ LIF scheme, i.e., optically pumping the argon ion $3 \mathrm{~d}^{2} \mathrm{G}_{9 / 2}$ metastable state to the $4 \mathrm{p}^{2} \mathrm{~F}_{7 / 2}^{0}$ state by $611.66 \mathrm{~nm}$ (vacuum wavelength) laser light and then detecting the $461.09 \mathrm{~nm}$ fluorescence photons that result from the decay of the $4 \mathrm{p}^{2} \mathrm{~F}^{0}{ }_{7 / 2}$ state to the $4 \mathrm{~s}^{2} \mathrm{D}_{5 / 2}$ state.

\subsection{LIF Measurements at Oblique Incidence}

One-dimensional argon ivdfs obtained $19 \mathrm{~cm}(z=169 \mathrm{~cm})$ downstream from the HELIX-LEIA junction for different laser injection orientations, $\beta$, in the $(\alpha, x)$ vertical plane (see Figure 4.23 (a) for the injection geometry) are shown in Figure 7.1. Iodine reference spectra, which indicate negligible drift of the laser, are shown along the top of each graph as well. At a first glance, the ivdfs obtained for laser injection parallel and 
Chapter 7: Oblique and Tomographic LIF measurements of the EDL in Argon Plasma

anti-parallel to the radial (or $x$ axis) direction, i.e., $\beta=0^{\circ}$ and $180^{\circ}$ (see Figures 7.1 (a) and (b)) indicate a single, nearly stationary, ion velocity distribution. The frequency shifts of the ivdfs obtained for $\beta=0^{\circ}$ and $180^{\circ}$ give flow velocities of $-80 \mathrm{~m} / \mathrm{s}$ and $+110 \mathrm{~m} / \mathrm{s}$, respectively. Both flow values are smaller than the uncertainty in the velocity determination. The ivdfs obtained for the other injection angles reveal a bimodal structure comprised of a slow ion population and a fast ion population whose drift velocity increases with the injection angle; reaching a maximum of $\sim-6.6 \mathrm{~km} / \mathrm{s}$ for $\beta=90^{\circ}$ (Figure $7.1(\mathrm{~g})$ ). Note that the laser light was injected towards the plasma source, so the negative ion flow velocity values are consistent with ion flow from HELIX into LEIA. The ion speeds shown in Figures 7.1 (a)-(g) are uncorrected for the projection of the laser along the $z$ axis, $\alpha=52^{\circ}$. The correction for projection along the $z$ axis was confirmed by injecting the laser along the $z$ axis from far end of HELIX (injection point $\mathrm{P}_{2}$ in Figure 4.24) and measuring the drift velocity of the fast ion distribution (as shown in Figure 7.2). The "passive" measurement (detailed in Chapter 4.4.) of the parallel speed of the fast ion population agree to within $5 \%$ of the oblique measurement after correction for projection angle. In Figure 7.2 (b), the positive frequency shift of the fast peak in the ivdf is again consistent with ion flow from the source into LEIA. 


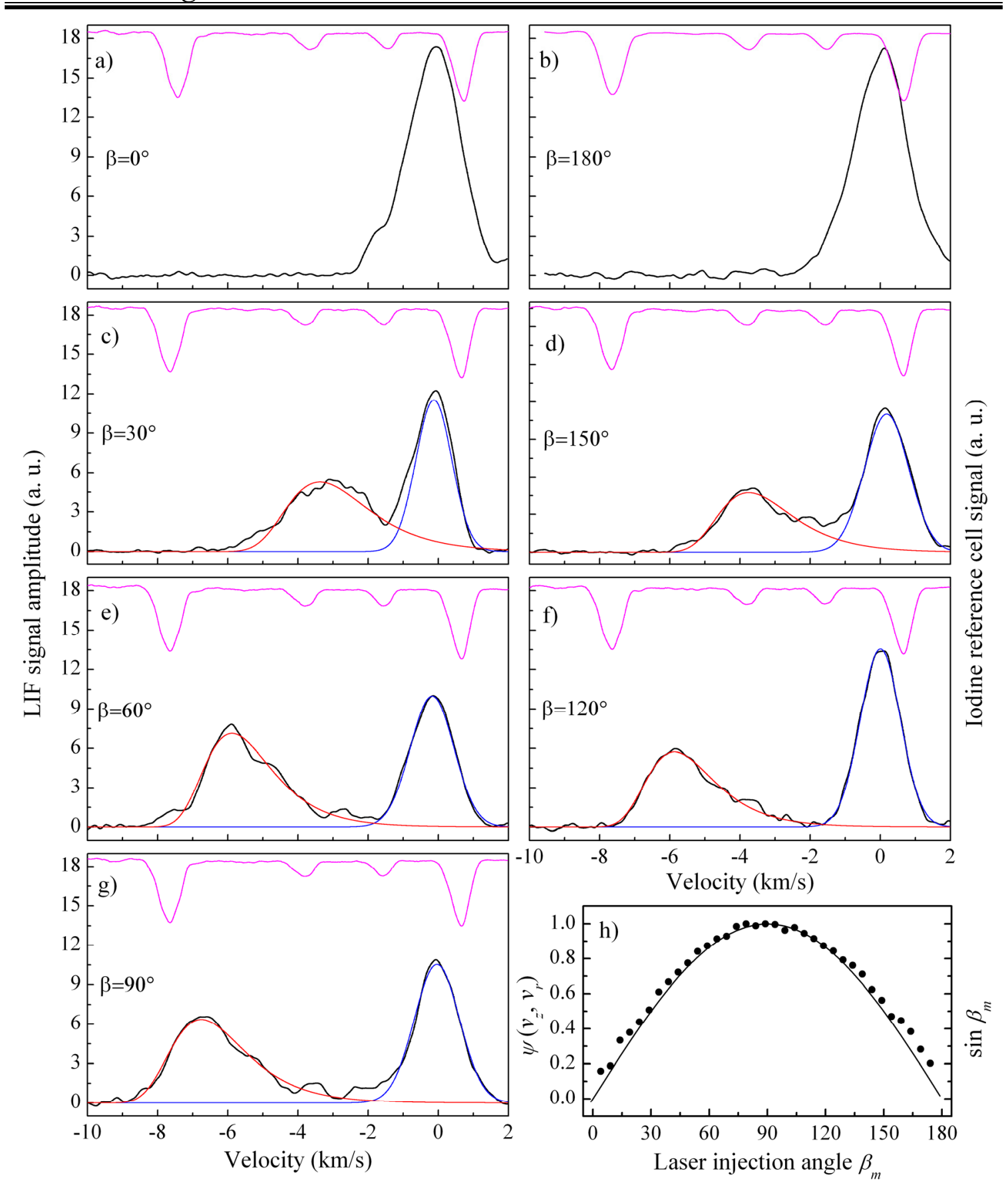

Figure 7.1. a-g) 1D argon ivdfs measured in the vertical plane $(\alpha, x)$ versus injection angle, $\beta$. The fast (red) and slow (blue) ion population ivdfs are obtained from a fit to the measurement. Iodine spectra obtained during each scan are shown across the top of each plot. h) Values of the function $\Psi$ for the best fit values of $v_{z}$ and $v_{r}$ (symbols) and $\sin \beta_{m}$ (line) 

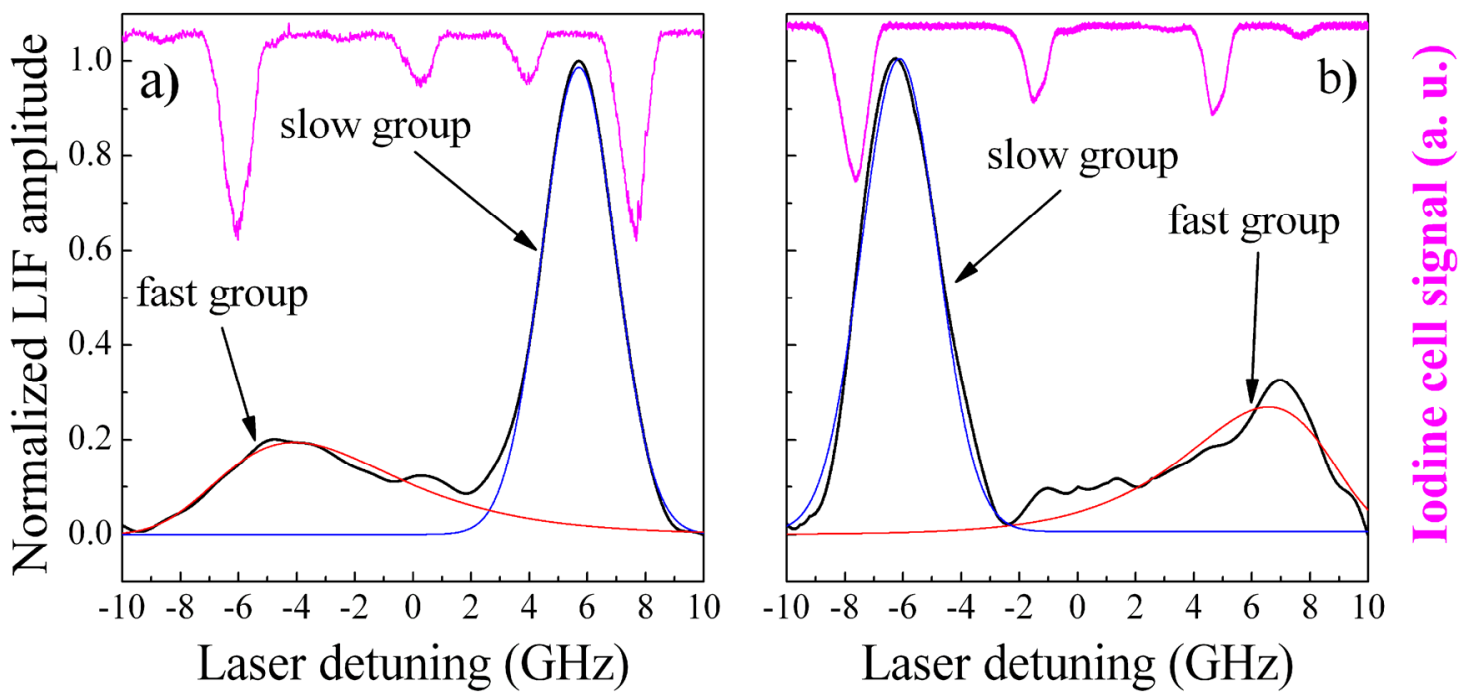

Figure 7.2. a) 1D LIF obtained in the "active" probe mode (the laser is injected and collected with the probe); b) 1D LIF obtained in the "passive" probe mode (the laser is injected from the end of HELIX and fluorescent emission collected with the probe)

For the geometry of Figure 4.23 (a), laser injection at angles $\alpha$ and $\beta$ with respect to the $z$ and $x$ axes, respectively, the maximum in the ivdf will occur at a velocity of

$$
v_{\alpha \beta}=-\left(v_{z} \cos \alpha+v_{y} \sin \alpha\right) \sin \beta-v_{x} \cos \beta,
$$

where the minus signs in the right-hand-side of the equation result from the assumption that the laser injection is opposite to the ion flow direction for all three coordinate directions. For a measurement location near the $z$ axis, the azimuthal flow can be neglected and assuming cylindrical symmetry

$$
v_{x}=v_{y}=v_{r} / \sqrt{2} .
$$

Substituting into Eq. (7.1) and defining $\zeta=v_{\alpha \beta} / v_{z}, \rho=v_{r} / \sqrt{2} v_{z}$, and $\psi=\sin \beta$,

$$
\left[(\cos \alpha+\rho \sin \alpha)^{2}+\rho^{2}\right] \psi^{2}+2 \zeta(\cos \alpha+\rho \sin \alpha) \psi+\zeta^{2}-\rho^{2}=0 .
$$

The solutions of Eq. (7.3) are given by

$$
\psi_{1,2}=\frac{-\zeta(\cos \alpha+\rho \sin \alpha) \pm \rho \sqrt{\rho^{2}+(\cos \alpha+\rho \sin \alpha)^{2}-\zeta^{2}}}{\rho^{2}+(\cos \alpha+\rho \sin \alpha)^{2}} .
$$

For $\alpha=90^{\circ}$ and $\alpha=0^{\circ}$, the solutions to Eq. (7.3) are always real. For any $v_{r}$ at any angle $\alpha$, the condition for a positive discriminant (the quantity under the square root) in Eq. (7.4) constrains the minimum value of $v_{z}$. Since $0^{\circ} \leq \beta \leq 180^{\circ}, \psi \geq 0$ in Eq. (7.4) 
requires a choice of \pm sign that depends on the relative directions of the axial flow velocity and the laser injection direction. With measurements of the ivdf at a couple of angles $\beta_{m}$, the axial $\left(v_{z}\right)$ and radial $\left(v_{r}\right)$ velocity components can be found by solving

$$
\psi-\sin \beta_{m}=0
$$

for $\zeta$ and $\rho$.

As shown above, the axial $\left(v_{z}\right)$ and radial $\left(v_{r}\right)$ velocities for the independent components of a bimodal distribution can be found in the general case of $v_{z} \neq v_{r} \neq 0$ by requiring $\psi\left(v_{r}, v_{z}\right)=\sin \beta_{m}$, for the measured ivdf and a known injection angle, $\beta_{m}$. Shown in Figure 7.1 (h) are the predicted values for $\psi\left(v_{r}, v_{z}\right)$ for the fast ion population. A slight departure from the ideal sinusoidal dependence evident near $\beta=0^{\circ}$ and $\beta=180^{\circ}$ is most likely due to imprecise determination of individual values (slow and fast) of $v_{\alpha \beta}$ at these injection angles due to overlap of the two distributions. We also find a small angular shift $\left(\sim 1^{\circ}\right)$ between $\psi$ and $\sin \beta_{m}$ caused by a slight imperfection in the angular alignment of the probe. We estimate the total uncertainty in the velocity components (including the uncertainties in fits to the ivdfs and in the absolute iodine spectral lines) to be $150 \mathrm{~m} / \mathrm{s}$. The best fit axial and radial velocity components for the fast ion group population are $v_{z}=$ $10.8 \mathrm{~km} / \mathrm{s}$ and $v_{r}=80 \mathrm{~m} / \mathrm{s}$, respectively. For the slow ion population, the best fit values for $v_{z}$ and $v_{r}$ are both smaller than the flow velocity uncertainty. Although the slow ion population is well fit with a single Gaussian distribution in Figure $7.1(\mathrm{c})-(\mathrm{g})$, the fast population has a long tail towards slower speeds. Since the projection of a Gaussian ivdf along a measurement direction remains a Gaussian function, as demonstrated by the Gaussian-like slow population distribution measurement, the long tail of straggling fast ions is not simply a projection artifact. Assuming that the slow ions are a background population created locally and the fast ions are created in the source and then accelerated by the EDL into the diffusion chamber, the long tail of the fast ion population is consistent with the slowing down of fast ions by elastic scattering and/or charge exchange collisions with the background gas. We note that similar asymmetrically distorted ivdfs, i.e. only half of the vdf is well-fit by a Gaussian function, were reported by Claire et al. for LIF observations of argon ions accelerated in an electrostatic presheath. ${ }^{7}$ To within experimental error, the integrated LIF signals corresponding to 
Chapter 7: Oblique and Tomographic LIF measurements of the EDL in Argon Plasma

Figures 7.1 (a) and (b) are equal to the sum of the integrated intensity peaks in Figure 7.1 (c)-(g). Therefore, it is reasonable to conclude that the ivdfs shown in Figures 7.1 (a) and (b) are two independent distributions that simply overlap in radial velocity space.

For the simpler case of a unimodal distribution, the bulk radial velocity is determined directly by injecting the laser along $x$ or $y$ direction. Then, with additional laser injection in a plane containing the direction of flow, $(y, z)$ for instance, $v_{z}$ can be found by inverting the velocity projection equation

$$
v_{\alpha}=-v_{z} \cos \alpha-v_{y} \sin \alpha .
$$

For $v_{z} \gg>v_{r} \cong 0$ (typically observed in our experiment for both the low and fast ion populations) and except for the case of $\alpha \cong 90^{\circ}$, when the injection angle coincides with the $y$ direction, the axial velocity becomes

$$
v_{z} \cong-v_{\alpha} / \cos \alpha .
$$

For the data shown in Figure $7.1(\mathrm{~g})$, the approximate analysis of Eq. (7.7) yields $v_{z} \cong$ $10.7 \mathrm{~km} / \mathrm{s}$; a difference of less than $1 \%$ from the value obtained with the more complete analysis. Since the fast population is not well fit by a simple Gaussian function, the parallel (axial) and perpendicular (radial) ion temperatures are not easily obtained from fits to the measurements. Best fit curves to the ivdfs, as shown in Figure 7.1 (c)-(g), are based on the LogNormal function provided in the OriginLab ${ }^{\circledR}$ fitting functions database. Although a FWHM for such distribution can be defined, it is not directly related to the thermodynamic temperature of the population. Taking the Gaussian-like left half of the distribution and mirroring it around the average speed of the fast population gives an $\alpha$ direction temperature of the fast ion group of $\sim 0.5 \mathrm{eV}$. From deconvolution of the $0^{\circ}$ and $180^{\circ}$ ivdfs, the radial temperature is $\sim 0.24 \mathrm{eV}$. Then, by Eq. (4.59), the axial temperature is $\sim 0.11 \mathrm{eV}$ and therefore the fast ion population is thermally anisotropic. From Maxwellian distribution fits to the slow ion population, the slow ion axial ion temperature is $\sim 0.22 \mathrm{eV}$ and the radial ion temperature is $\sim 0.18 \mathrm{eV}$. Since the difference is within the experimental uncertainty, the slow population ion temperatures are consistent with an isotropic slow ion population. 


\subsection{Experimental Difficulties Associated with LIF Tomography}

LIF tomography requires multiple 1D ivdfs measurements obtained at different laser injection angles. Because the velocity resolution in a reconstructed 2D ivdf is inversely proportional to the number of laser injection directions $\delta V / V=\pi / 2 I^{8}$ (with $\delta V$ the minimum resolvable feature size and $V$ the velocity range), too few laser injection directions yield an unreliable reconstruction (see Figure 7.3 (a)). However, too many laser injection directions require an unreasonably long measurement time. Yielding $\sim 10 \%$ resolution, 18 injection angles are sufficient for our experimental conditions. For a laser scan rate of $0.3-0.5 \mathrm{GHz} / \mathrm{s}$ for a $20 \mathrm{GHz}$ range and averaging over $2-5$ scans to improve the signal-to-noise of each 1D measurement, the total time to collect $181 \mathrm{D}$ ivdfs ranges from 30 to $90 \mathrm{~min}$. During this interval, the plasma conditions and laser stability must be maintained. As shown in Figure 7.3 (b), an uncorrected laser frequency drift of less than $0.3 \mathrm{GHz}$ during only four of the 1D ivdfs can compromise the entire reconstruction process and introduce "ghost" features. Notwithstanding the technical difficulties, LIF tomography does reveal subtle ivdf features that cannot be identified in 1D ivdf measurements.
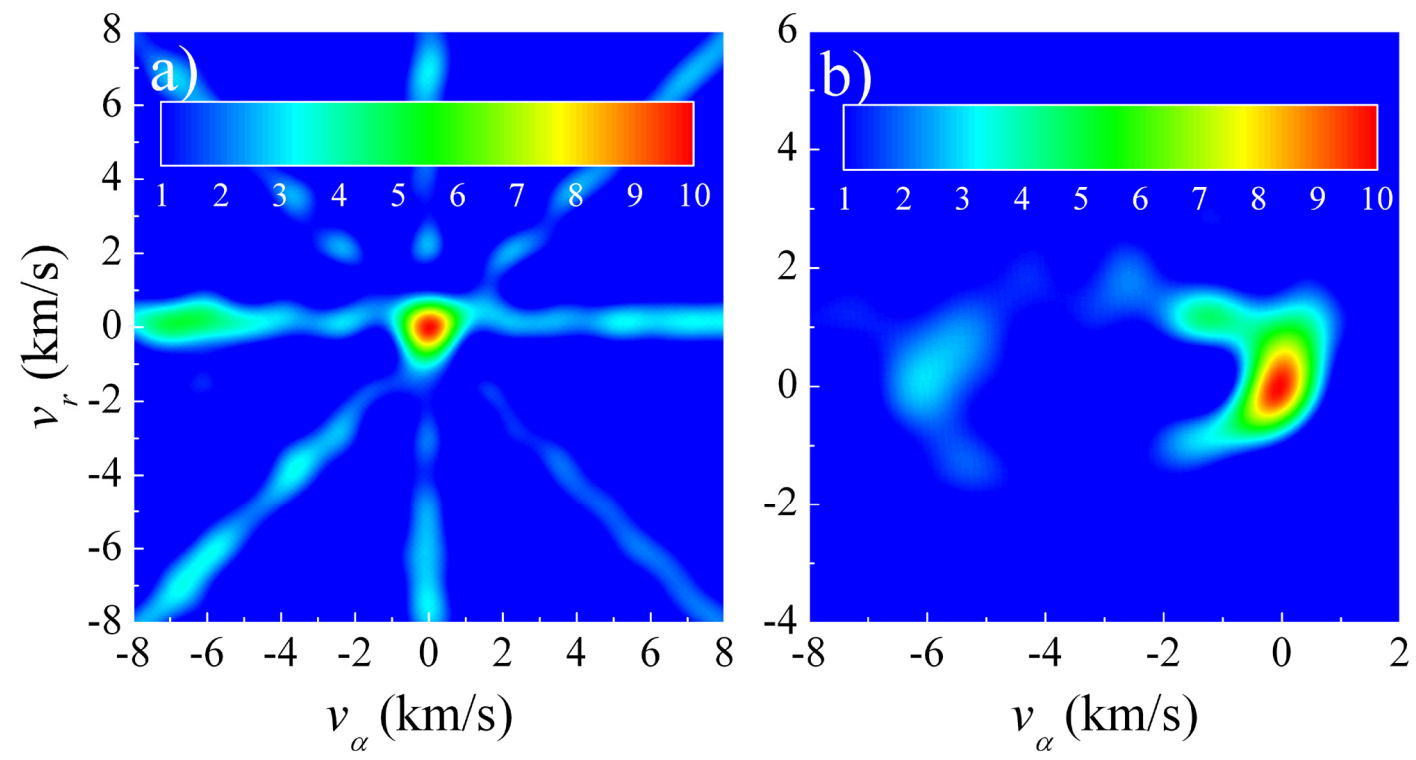

Figure 7.3. Examples of poor tomographic inversions: a) an insufficient number of laser injections leading to "corners" in the final reconstructed vdf; b) laser drift during 1D collection resulting in ghost features and a distorted $2 \mathrm{D}$ ivdf. 


\subsection{D Ion Velocity Distribution Functions}

Shown in Figure 7.4 is a complete 2D ivdf obtained from the full set of 1D ivdfs presented in Figure 7.1. The probe was rotated in $5^{\circ}$ increments, i.e., 36 injection directions, over $\pi$ radians. For each injection direction, the 1D ivdf was obtained from an average of four individual measurements. Although fairly smooth, the background of the 2D ivdf image shows some evidence of artifacts resulting from the filtered back projection process (the small hills and valleys in the surface plot of Figure 7.4). The artifact amplitudes are less than $10 \%$ of the height of the primary peak.

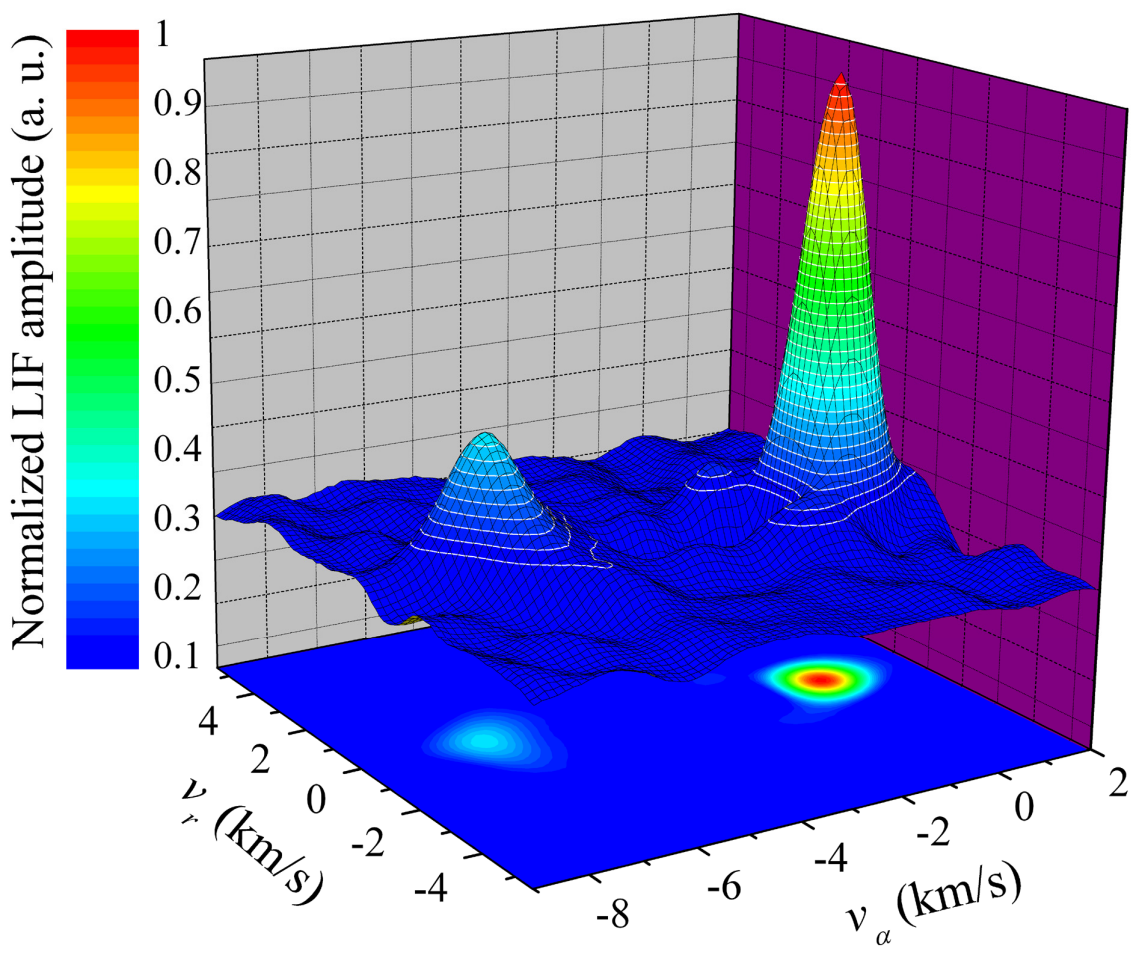

Figure 7.4. 2D bimodal ivdf obtained $19 \mathrm{~cm}$ downstream the HELIX-LEIA junction showing the fast and slow ion populations. The LIF signal is normalized to the slow group LIF intensity. The negative value for the fast group axial velocity arises from ion flow out of the source towards the direction from which the laser is injected.

To improve the accuracy of the reconstruction, each 1D ivdf measurement was adjusted for laser drift before processing with the back-projection algorithm. Consistent with the 1D measurements, the tomographic images indicate a bimodal distribution consisting of a nearly stationary background ion population and a fast ion population ${ }^{9}$ 
Chapter 7: Oblique and Tomographic LIF measurements of the EDL in Argon Plasma

(velocities along the $\alpha$ direction of $\sim-110 \mathrm{~m} / \mathrm{s}$ and $\sim-6.6 \mathrm{~km} / \mathrm{s}$, respectively). After corrections with Eq. (7.7), the axial velocities are determined to be $\sim 180 \mathrm{~m} / \mathrm{s}$ for the bulk population and $\sim 10.7 \mathrm{~km} / \mathrm{s}$ for the fast population. From the integrated velocity space volumes, we estimate the relative fast to bulk population ratio to be 1:3. As noted previously, the fast ion population is a clear signature of an EDL upstream of the measurement location.

Subtle features of the ivdf are more easily identified in the magnified tomographic images of the slow and fast populations shown in Figure 7.5. Each plot in Figure 7.5 is a 300 point $\times 300$ point section of the original 800 point $\times 800$ point image. To the limit of the reconstruction process, the slow group ivdf is isotropic with a ratio of the full widths at half maximum (FWHMs) in the radial and $\alpha$ directions $\left(\Delta v_{r} / \Delta v_{\alpha}\right)_{\text {FWHM }}$ of 0.93. Based on Eq. (4.59), the radial and axial ion temperatures are $0.13 \mathrm{eV}$ and $0.18 \mathrm{eV}$, respectively.

Consistent with the previous 1D measurement analysis, the fast population ivdf is decidedly not isotropic. The ivdf has a triangular shape with "tails" aligned along directions roughly midway between the radial and $\alpha$ directions. The shapes of the slow and fast 2D ivdfs are consistent with different origin locations for the two ion populations: the slow group is a locally created background population whereas the fast population is created in the source, undergoes acceleration through the potential drop of the EDL, and has its ivdf broadened by collisions experienced during the transit to the measurement location. The symmetrical stretching towards lower absolute speeds, indicated by the dashed white lines in Figure 7.5 is inconsistent with upstream perpendicular heating followed by an adiabatic upwelling; the mechanism responsible for the "ion conics" observed in auroral EDLs and in laboratory experiments. ${ }^{10,11,12}$ Not only is the $\alpha$ direction not equivalent to the magnetic field direction, but the orientation of the conic shape of the $2 \mathrm{D}$ ivdf is opposite to what would be expected for a conversion of the upstream perpendicular energy into downstream parallel energy based on magnetic moment conservation in the expansion region. In typical ion conic formation, ions with large perpendicular energy in regions of stronger magnetic field strengths appear as ions with larger parallel energy in regions of weaker magnetic field strength. Therefore, the tip of the cone should point toward smaller absolute speeds. However, in these measurements, the ions with the largest radial velocities also have the slowest velocities 
along the $\alpha$ direction. This stretching towards lower speeds along the $\alpha$ direction could indicate increased collisional drag for the higher speed ions, increased pitch-angle scattering, or the action of some other ion acceleration mechanism. ${ }^{13}$

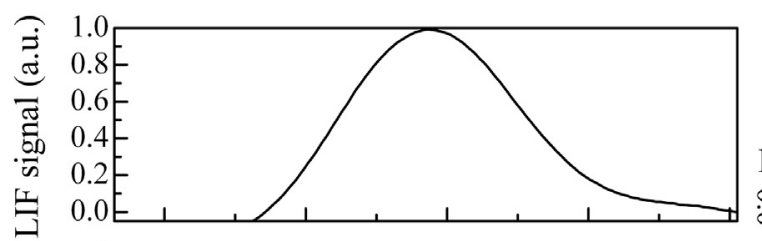

a)

LIF signal (a. u.)
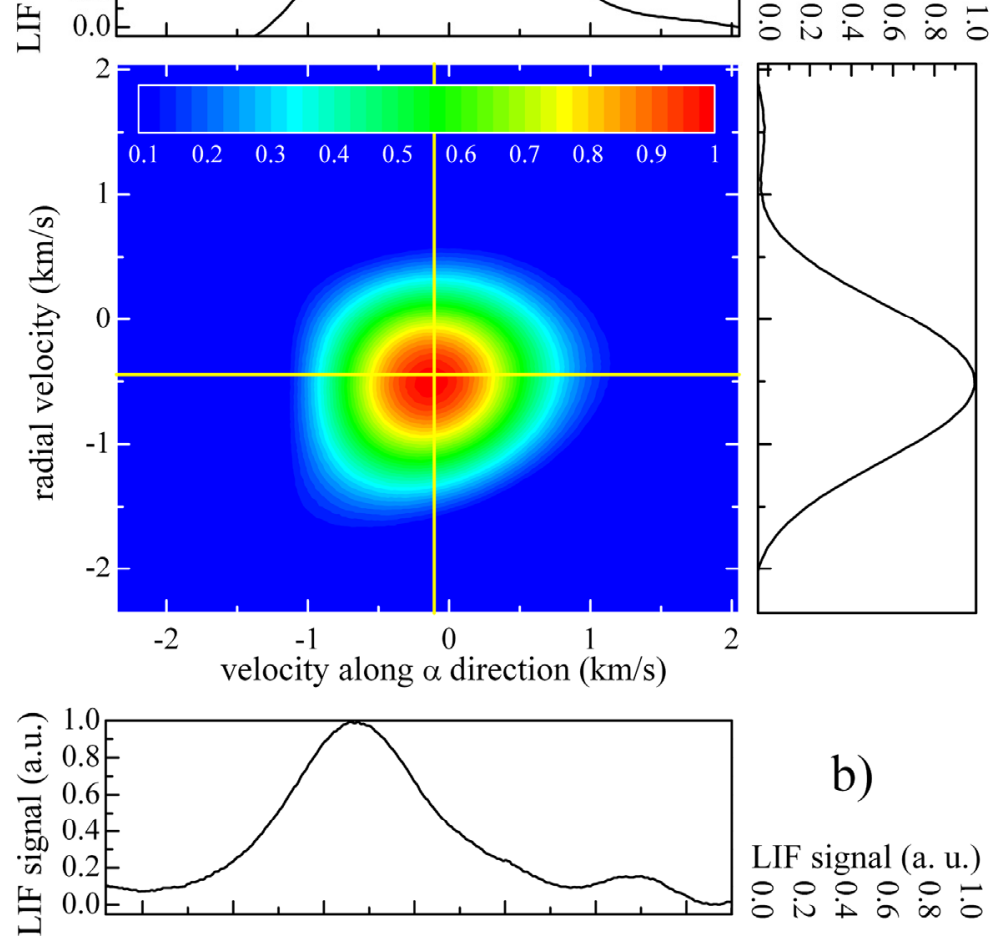

b)

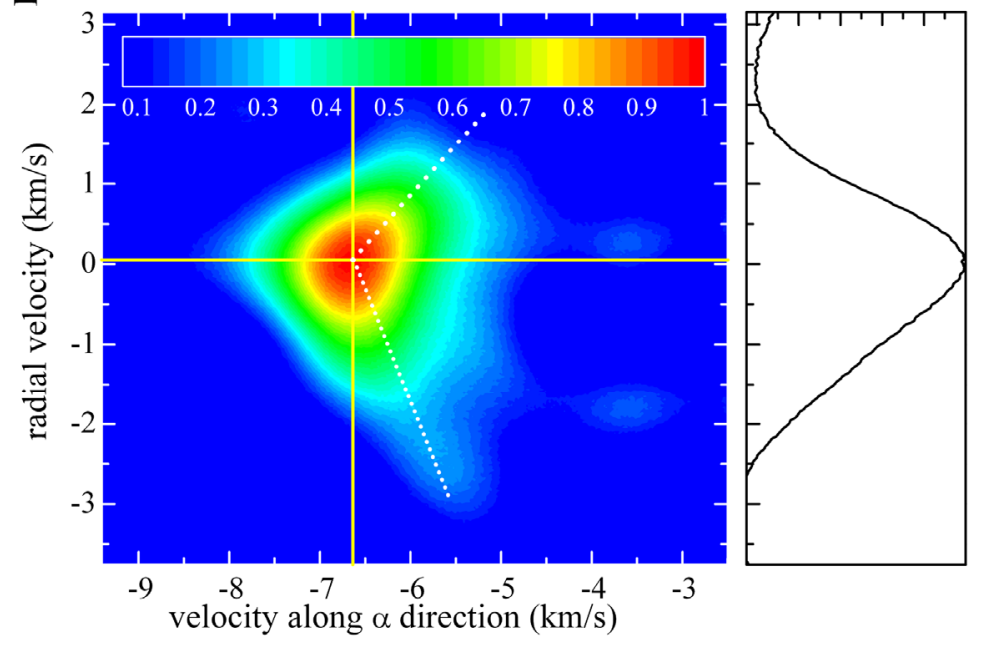

Figure 7.5. Expanded views of sections of tomographic image shown in Figure 7.4 for the (a) slow ivdf and the (b) fast ivdf. The color bar in both plots is the normalized LIF signal intensity. The top and side graphs are horizontal and vertical slices (along yellow lines) through the 2D ivdf at the center of the ion distribution. 
Chapter 7: Oblique and Tomographic LIF measurements of the EDL in Argon Plasma

The bulk radial velocities obtained from the tomographic analysis are approximately $-450 \mathrm{~m} / \mathrm{s}$ and $+150 \mathrm{~m} / \mathrm{s}$ for the slow and fast populations, respectively. This small but statistically significant difference could indicate a slight misalignment of the axes of the HELIX and LEIA systems or a bend in the probe shaft relative to the chamber axis that was not detected in the 1D ivdf measurements.

An important difference between these measurements and 2D LIF tomographs of an ion beam obtained in an electrostatic presheath [8], is that in these measurements, only a faint tail of ions is seen extending from the beam velocity to slower speeds. In the presheath measurements, a continuous population of ions extending from the highest velocity of $\sim 6 \mathrm{~km} / \mathrm{s}$ all the way down to zero velocity was observed (see Figures $2 \mathrm{~d}$ and $2 e$, in Ref. [8]). Since LIF detects ions in the $3 \mathrm{~d}^{2} \mathrm{G}_{9 / 2}$ metastable state, only a small fraction of the fast ion population travelling from HELIX into LEIA is detectable by LIF. Given a quenching cross-section for collisions of the $3 d^{2} \mathrm{G}_{9 / 2}$ state with ground state neutral argon of $1 \times 10^{-14} \mathrm{~cm}^{2}{ }^{14}$ the quenching mean free path $(\mathrm{mfp})$ is $17 \mathrm{~cm}$; less than half of the charge-exchange mfp of $36 \mathrm{~cm}$ assuming $\sigma_{\mathrm{CX}}=4.7 \times 10^{-15} \mathrm{~cm}^{2}$ for the measured ion energies. ${ }^{15}$ Thus, an ion in the metastable state will be depopulated by quenching long before significant velocity changes result from charge exchangecollisions. That metastable quenching dominates over charge-exchange losses is demonstrated by the two tomographic images of an ion beam shown in Figure 7.6. Both were obtained under identical plasma conditions and at two axial locations separated by 9 $\mathrm{cm}$. There is no significant change in the axial velocity of the fast population $\left(\mathrm{V}_{\mathrm{za}} \cong \mathrm{V}_{\mathrm{zb}} \cong\right.$ $10.3 \mathrm{~km} / \mathrm{s}$ ) but there is a factor of 1.5 decrease in LIF intensity.

Thus, the principle difference between these measurements and those reported in Ref. [8] is that in this case, metastable quenching prevents the slower ions from being detected by LIF after traveling from the upstream acceleration region. In conclusion, 1D LIF investigations at oblique incidendece relative to the ion flow direction can provide a comphrensive picture of the ivdf in an argon plasma. Even in the case of a bimodal distribution, the radial and axial velocities and temperatures for both populations of ions can be determined. 
Chapter 7: Oblique and Tomographic LIF measurements of the EDL in Argon Plasma

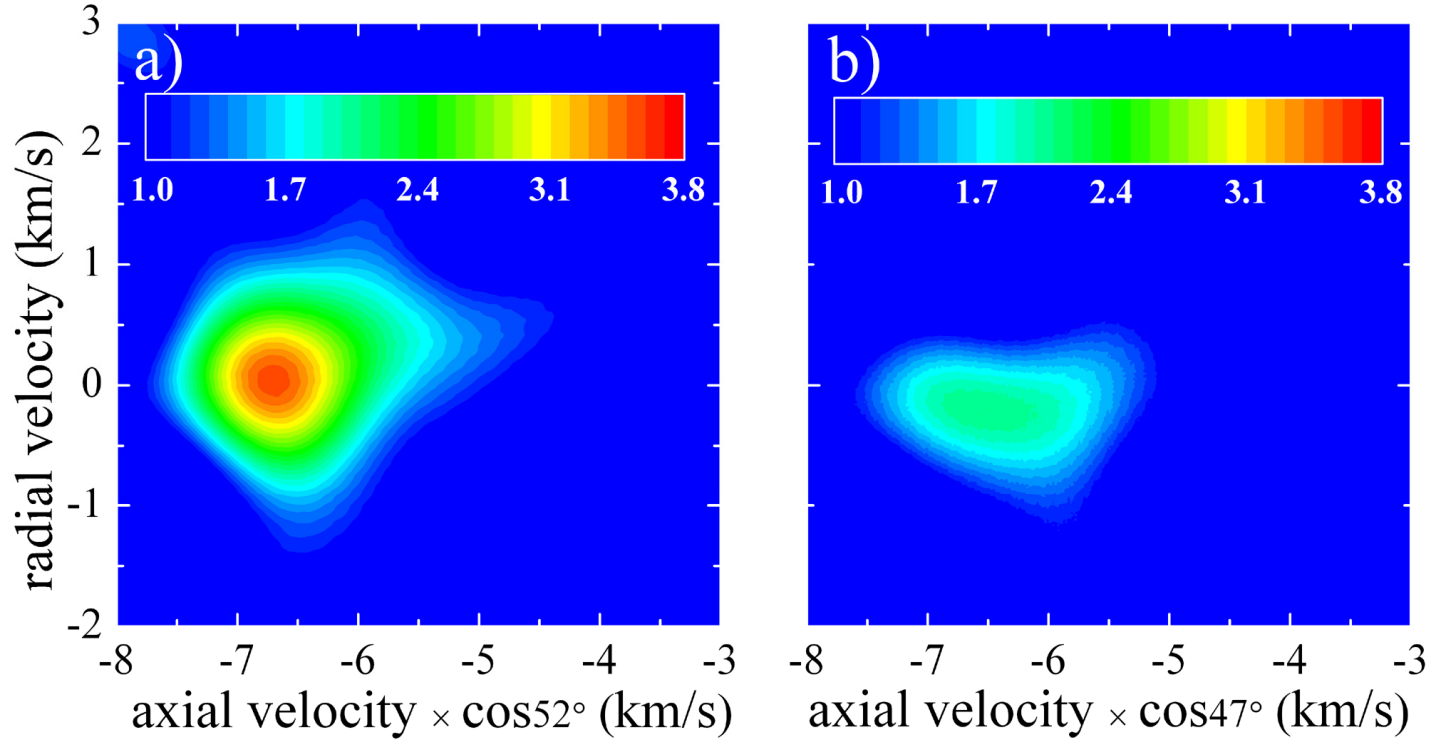

Figure 7.6. Tomographic images of the fast ion population $19 \mathrm{~cm}$ a) and $28 \mathrm{~cm} \mathrm{~b}$ ) downstream from HELIX-LEIA junction for identical plasma operating conditions. To emphasize the difference in LIF amplitudes, the same color bar is used for both graphs.

The LIF measurements are consistent with a locally generated, nearly-stationary, background ion population and fast population created at an EDL upstream of the measurement location. Tomographic LIF measurements, also at oblique incidence, demostrate the significant anistropy of the fast ion population, confirm that metastable quenching is the dominant mechanism for reducing the LIF signal amplitude of the fast ion population in expanding helicon plasmas, and may provide evidence of pitch-angle scattering for the most energetic ions. 
Chapter 7: Oblique and Tomographic LIF measurements of the EDL in Argon Plasma

\section{Chapter 7 References}

${ }^{1}$ W. M. Ruyten and D. Keefer, AIAA Jour. 31, 2083 (1993)

${ }^{2}$ N. Sadeghi, T. Nakano, D. J. Trevor, and R. A. Gottscho, J. Appl. Phys. 70, 2552 (1991)

${ }^{3}$ E. Scime, R. Hardin, C. Biloiu, A. M. Keesee and X. Sun, Phys. Plasmas 14, 043505 (2007)

${ }^{4}$ E. W. Reynolds, T. Kaneko, M. Koepke, and R. Hatakeyama, Phys. Plasmas 12, 072103 (2005)

${ }^{5}$ C. Charles, Plasma Sources Sci. Technol. 16, R1 (2007)

${ }^{6}$ C. Biloiu, X. Sun, E. Choueiri, F. Doss, E. Scime, J. Heard, R. Spektor, and D. Ventura, Plasma Sources Sci. Technol. 14, 359 (2005)

${ }^{7}$ N. Claire, G. Bachet, U. Stroth and F. Doveil, Phys. Plasmas 13, 062103 (2006)

${ }^{8}$ D. C. Zimmerman, R. McWilliams, and D. A. Edrich, Plasma Sources Sci. Technol. 14, 581 (2005)

${ }^{9}$ I. A. Biloiu, E. E. Scime, C. Biloiu and S. A. Cohen, IEEE Trans. Plasma Sci. 36, 1216 (2008)

${ }^{10}$ J. E. Borovsky, J. Geophys. Res. 89, 2251 (1984)

${ }^{11}$ T. Chang, G. B. Crew, J. M. Retlerer, and J. R. Jasperse, IEEE Trans. Plasma Sci. 17, 186 (1989)

${ }^{12}$ M. Zintl, R. McWilliams, and N. Wolf, Phys. Plasmas 2, 4432 (1995)

${ }^{13}$ I. A. Biloiu, E. E. Scime and C. Biloiu, Appl. Phys. Lett. 92, 191502 (2008)

${ }^{14}$ F. Skiff, G. Bachet, and F. J. Doveil, Phys. Plasmas 8, 3139 (2001)

${ }^{15}$ A. V. Phelps, J. Chem. Ref. Data 20, 557 (1991) 


\section{Chapter 8: Summary and Conclusions}

Electron energy distribution functions and ion velocity distribution functions in the HELIX-LEIA system presented in this work confirm the existence of an electric double layer in the magnetic field divergent region of a freely expanding single- and multi-component helicon plasma.

Langmuir probe measurements in mixed Ar-Xe plasma revealed that eedfs exhibit a transition from a Maxwellian type electron distribution in pure argon plasma to a Druyvesteyn type electron distribution for xenon fractions of a few percent. Another striking change of the eedf shape is the depletion of the high energy tail of the distribution with increasing xenon fraction. The maximum electron energies in the tail decrease by $\sim 15 \mathrm{eV}$ for pure xenon compared to pure argon. Both in the helicon source and in the expansion region, due to lower ionization potential and larger ionization crosssections, increasing xenon fraction leads to an exponential decrease of the electron temperature. Although the term "electron temperature" has no physical meaning for distributions other than Maxwellian, in the sense of mean electron energy, in HELIX the effective electron temperature decreases from $6.5 \mathrm{eV}$ in pure argon to $3.8 \mathrm{eV}$ in pure xenon. Similar variation is observed in LEIA, the electron temperature decreases from $7.2 \mathrm{eV}$ to $4.7 \mathrm{eV}$. Electron densities calculated from eedfs integrations show a linear increase with increasing xenon fraction: in HELIX the density increases from $1.07 \times 10^{11}$ $\mathrm{cm}^{-3}$ up to $1.25 \times 10^{11} \mathrm{~cm}^{-3}$ whereas in LEIA the density increases from $2 \times 10^{9} \mathrm{~cm}^{-3}$ up to $5 \times 10^{9} \mathrm{~cm}^{-3}$. Due to the limited number of ports, probe diagnosis is limited in HELIX. Future experiments could benefit from an axially scanning Langmuir probe. ${ }^{1}$ Although challenging to construct because of the small dimensions necessary to avoid perturbing the plasma, such a tool would allow determination of the location of the EDL with high precision $^{2}$ and might even provide direct evidence of the expected high energetic electron population streaming through the EDL into HELIX.

Optical emission spectroscopy investigation of argon and xenon neutrals enabled estimation of neutral gas relative densities and correlation with individual gas flow rates. From the Ar $811.75 \mathrm{~nm}$ and Xe $823.39 \mathrm{~nm}$ emission line intensities, the computed excitation rate coefficients from experimentally determined eedfs and excitation cross- 
sections available in literature, and a Corona model, the Ar and Xe neutral densities were determined as a function of Xe fraction. Similarly, from the $\mathrm{Ar}^{+} 434.93 \mathrm{~nm}$ and $\mathrm{Xe}^{+}$ $529.37 \mathrm{~nm}$ emission lines and the computed ionization rate coefficients, an OES analysis of ionic/neutral emission lines intensity ratios enabled the calculation of partial $\mathrm{Ar}^{+}$and $\mathrm{Xe}^{+}$densities. Although the sum of computed densities did not match the electron density value determined from probe measurements, the analysis provided a qualitative indication of species ionization as a function of xenon fraction. For future research, it would be useful to have a vertically elongated window mounted in the vicinity of $z=$ $140-150 \mathrm{~cm}$ for OES investigations. Through Abel inversion, ${ }^{3}$ the ion and neutral radial density profiles could then be determined and related to possible EDL spatial structures. ${ }^{4}$

For pure argon plasma, laser induced fluorescence revealed bimodal ion velocity distribution functions in the magnetic expansion region of the HELIX-LEIA device. The ivdfs were comprised of a slow moving ion group and a fast, highly supersonic $\left(\sim 2.9 c_{\mathrm{Ar}+}\right)$ ion group. By decreasing the magnetic field in the diffusion chamber and holding constant the magnetic field in the source, i.e., by increasing the divergence of the magnetic field in the expansion region, the ratio of the heights of the fast and slow ion distributions increased exponentially from $\sim 0.4$ to $\sim 1.7$. The parallel speed of the fast component of the velocity distribution also increased, reaching a maximum of $\sim 10.8$ $\mathrm{km} / \mathrm{s}$ while the speed of the slower component was essentially unchanged. Similar bimodal ivdfs, but with the fast ion group flowing at much lower speed $(\sim 6 \mathrm{~km} / \mathrm{s})$ were detected by LIF close to the HELIX- LEIA junction, just $4 \mathrm{~cm}$ inside the source. These observations confirmed the different origin of the two ion groups: the slow ion group is a population created locally and the fast ion group is created in the source and then accelerated through the EDL. Because the ivdf just before magnetic expansion region is insensitive to variations in the magnetic field divergence and because the difference in parallel kinetic energy is too large $(\sim 15 \mathrm{eV})$ to be explained by conversion of the upstream radial and/or azimuthal kinetic energy, an acceleration mechanism or mechanisms in addition to the EDL must be present in these plasmas. To study the acceleration mechanisms individually, an interesting experiment would be to modify the cross-sectional area of the helicon source close to the HELIX-LEIA junction by inserting conductive cylinders of different opening diameters. Such an experiment would be 
similar to the aperture experiments of Sun et al. ${ }^{5}$ and would help elucidate the effect of the physical expansion ratio on the parallel flow speed. ${ }^{6}$

Ion velocity distribution functions in two positive ion species plasmas were studied for two binary gas mixtures: Ar-Xe and Ar-He. Due to the lack of a LIF scheme for $\mathrm{He}^{+}$, only $\mathrm{Ar}^{+}$and $\mathrm{Xe}^{+}$ivdfs were investigated. For $\mathrm{Ar}^{+}$we used the classical $3 \mathrm{~d}^{2} \mathrm{G}_{9 / 2}$ $\rightarrow 4 \mathrm{p}^{2} \mathrm{~F}_{7 / 2}^{0} \rightarrow 4 \mathrm{~s}^{2} \mathrm{D}_{5 / 2}$ three level LIF scheme for which the transition wavelengths, level energies, and Zeeman splitting corrections are known with high precision. However, the $5 \mathrm{~d}^{4} \mathrm{D}_{7 / 2} \rightarrow 6 \mathrm{p}{ }^{4} \mathrm{P}_{5 / 2} \rightarrow 6 \mathrm{~s}{ }^{4} \mathrm{P}_{5 / 2}$ LIF scheme used for $\mathrm{Xe}^{+}$was not so well understood.

First, significant differences in the precise rest frame $\mathrm{Xe}^{+}$line wavelength exist in the literature. A wave number difference of $\sim 0.08 \mathrm{~cm}^{-1}$ found between two reports that use this LIF scheme translates into a substantial error of $\sim 1.5 \mathrm{~km} / \mathrm{s}$ when converted to ion velocity. ${ }^{7,8}$ From our measured on-axis perpendicular LIF signal deep in LEIA, and assuming zero radial velocity, we determined the wave number of the $\mathrm{Xe}^{+}$transition to be $16521.332 \mathrm{~cm}^{-1}$. Second, because of isotopic spitting and non-zero nuclear spin of odd isotopes 129 and 131, the $\mathrm{Xe}^{+}$fluorescence line has a very complicated structure.

In the case of Ar-Xe plasma, we found that in the source both the $\mathrm{Ar}^{+}$and $\mathrm{Xe}^{+}$ ivdfs are unimodal. Their parallel speeds $\left(\sim 1.7 \mathrm{~km} / \mathrm{s}\right.$ for $\mathrm{Ar}^{+}$and $\sim 1 \mathrm{~km} / \mathrm{s}$ for $\left.\mathrm{Xe}^{+}\right)$are subsonic and unaffected by the change in the gas composition. The fact that their speed ratio scales inversely proportional with ion mass ratio indicates a slight axial potential gradient. Close to the HELIX-LEIA junction, at $z=146 \mathrm{~cm}$ and for a very narrow range of gas composition ( $0-4 \%$ xenon fraction), the argon ivdf shows a bimodal structure, indicative of an EDL upstream of the measurement location. The fast ion component has a parallel speed that increases linearly with the xenon fraction from $\sim 6.7 \mathrm{~km} / \mathrm{s}$ in pure argon to $\sim 8 \mathrm{~km} / \mathrm{s}$ for a $4 \%$ xenon fraction. These values are well above the argon ion or system sound speeds $(\sim 4 \mathrm{~km} / \mathrm{s})$. The slow ion component has a parallel speed of $\sim 2.5$ $\mathrm{km} / \mathrm{s}$ and is not affected by the small change in the gas composition. The LIF amplitudes of both ion groups decrease sharply with increasing xenon fraction. Above a xenon fraction of 4\%, the lock-in amplification could no longer discriminate the LIF signal from the noise. The simultaneous sharp drop in the measured electron temperature suggests a weak production rate of the $3 d^{2} \mathrm{G}_{9 / 2}$ metastable state rather than very efficient destruction. Additional ivdfs taken 1 and $2 \mathrm{~cm}$ downstream of the $z=146 \mathrm{~cm}$ location 
show a slight increase in the speed of the fast group, an indicative of a second acceleration mechanism. Deep in LEIA, the bimodal character of the $\mathrm{Ar}^{+}$ivdf is maintained. For the narrow 0 to $3 \%$ xenon fraction range for which the $\mathrm{Ar}^{+}$ivdf is detectable, both slow and fast ion population speeds are not affected by the change in the gas composition. As was found in pure argon, the parallel flow speed of the fast ion component $\left(\sim 10.5 \mathrm{~km} / \mathrm{s} \cong 2.6 c_{\mathrm{Ar}+}\right)$ is larger at $z=169 \mathrm{~cm}$ than at $z=146 \mathrm{~cm}$. The $\sim 8-13$ $\mathrm{eV}$ difference in parallel kinetic energy cannot be explained solely by radial or azimuthal kinetic energy conversion based on magnetic moment conservation. Therefore, the additional ion acceleration has to result from other mechanism(s) such as magnetic and/or geometric expansion. Although EDL presence is confirmed by the bimodal nature of the $\mathrm{Ar}^{+}$ivdf at $z=146 \mathrm{~cm}$, the $\mathrm{Xe}^{+}$ivdf is unimodal. Since the $\mathrm{Ar}^{+}$and $\mathrm{Xe}^{+} \mathrm{LIF}$ measurements do not encompass a common gas mixture range, it is possible that at moderate pressures the EDL does not form for xenon fractions above a threshold value. Previous experiments have found that in pure xenon plasma, EDL formation requires a much lower pressure ( $\sim 07$ mTorr) than in argon ( 1.5 mTorr) ${ }^{9}$

Because the investigated $5 \mathrm{~d}^{4} \mathrm{D}_{7 / 2} \mathrm{Xe}^{+}$metastable state is a relatively low energy state $(11.83 \mathrm{eV})$, the $\mathrm{Xe}^{+}$LIF signal is detectable down to a xenon fraction of $13 \%$. Over a xenon fraction range of 0 to $13 \%$, the parallel flow speed increased slightly from $\sim 1.3$ $\mathrm{km} / \mathrm{s}$ in pure xenon to $\sim 2.2 \mathrm{~km} / \mathrm{s}$ for a xenon fraction of $13 \% .2 .2 \mathrm{~km} / \mathrm{s}$ is slightly larger than the xenon ion sound speed, but smaller than the system sound speed. If an EDL forms at the end of the plasma source in a mixed gas plasma, the kinetic energy of both species should reflect the energy gain of passing through the EDL. Although uniquely capable of identifying the behavior of each ion species in the EDL (a RFEA is unable to distinguish between the two ion species), the lack of a fast ion component in the $\mathrm{Xe}^{+}$ivdf at $z=146 \mathrm{~cm}$ for xenon fractions for which fast ions were observed in the $\mathrm{Ar}^{+}$ivdf prevented us from confirming the EDL strength implied by $\mathrm{Ar}^{+}$ivdf measurements. Improved rf coupling is needed to transfer more power to the plasma; increasing the overall ion densities and thereby the ion metastable state densities as well. Only then will it be possible to perform simultaneous LIF measurements on both ion species over a range of gas compositions. Through such experiments, the sensitivity of EDL formation and strength to gas composition could be determined. 
As a result of the large differences in the ionization potentials and the ionization cross-section peak values of argon and helium, changing the gas composition in $\mathrm{Ar}-\mathrm{He}$ plasma produces large variations in both electron temperature and plasma density. Probe measurements found a two fold increase in the electron temperature with increasing helium fraction, from $\sim 7 \mathrm{eV}$ for pure argon plasma up to $\sim 14 \mathrm{eV}$ for a helium fraction of $80 \%$. Over the same helium fraction range, the electron density drops by more than three orders of magnitude, from $1.14 \times 10^{11} \mathrm{~cm}^{-3}$ down to $6.5 \times 10^{7} \mathrm{~cm}^{-3}$. Computed ion densities based on measured electron density and calculated ionization rate coefficients for each ion species suggest that even at a helium fraction of $80 \%$, argon ions significantly outnumber helium ions.

As observed in Ar-Xe plasma, the LIF measured $\mathrm{Ar}^{+}$ivdf in Ar-He plasma at the end of the helicon source exhibits a bimodal structure with fast and slow ion populations. However, in Ar-He plasma, the LIF signal is detectable up to helium fractions of $30 \%$. With increasing helium fraction, the parallel flow speed of the fast $\mathrm{Ar}^{+}$population increased from $\sim 5.2 \mathrm{~km} / \mathrm{s}$ at $0 \%$ helium fraction to $\sim 7.8 \mathrm{~km} / \mathrm{s}$ at a helium fraction of $30 \%$. As the helium fraction increased, the slow argon ion population changed from a single Gaussian to a wide distribution extending all the way from the speed of the fast population to $0 \mathrm{~m} / \mathrm{s}$ - a shape characteristic of a distribution of particles slowing down due to charge-exchange collisions. The larger $\mathrm{Ar}^{+}$flow speeds observed with increasing helium fraction might result from the same additional acceleration mechanism postulated to be at work in pure Ar and Ar-Xe plasmas. An effective $\mathrm{He}^{+}$LIF scheme would allow comparison of the $\mathrm{Ar}^{+}$and $\mathrm{He}^{+}$parallel flow speeds and provide critically needed additional information about the ion acceleration mechanisms. Although unfruitful until now, ${ }^{10}$ WVU helicon group research into the infrared $1012 \mathrm{~nm} \mathrm{He}^{+}$line might yet yield a breakthrough in $\mathrm{He}^{+}$LIF development.

Time resolved laser induced fluorescence measurements with $1 \mathrm{~ms}$ resolution provided important insight into the temporal evolution of the argon ion velocity distribution function in the expansion region of a pulsed helicon plasma. $19 \mathrm{~cm}$ downstream of the helicon source in pulsed discharges, the ivdf has a bimodal structure; indicative of an EDL upstream of the measurement location. The slow population forms as soon as the rf pulse is applied and persists for few ms after the rf pulse terminates. The 
fast population LIF signature appears few ms after the rf pulse begins. Varying the pulse duration by either modifying the pulse repetition frequency or the duty cycle did not affect the parallel velocities of the slow and fast ion populations. The time delay in the appearance of the fast ion population depends on the pulse repetition frequency and the rf duty cycle. For higher pulse repetition frequencies and/or longer duty cycles, the fast ion population appears more quickly. Simultaneous RFEA measurements would be very useful in identifying the origin of this time lag. If the same time lag appears in RFEA measurements, the RFEA measurements would confirm that the EDL forms later in the pulse.

Although laborious, the method described for determining perpendicular and parallel ivdfs from LIF measurements at oblique laser incidence (with respect to the magnetic field direction) can be useful for many plasma experiments where optical access is limited. This method eliminates the need to ensure that the laser light is injected either perfectly parallel or perfectly perpendicular to the ambient magnetic field. For simple plasma geometries and/or for LIF investigation locations for which the perpendicular component can be neglected, the parallel component is simply a projection of the 1D ivdf along the laser propagation direction. ${ }^{11}$

Tomographic LIF measurements, also at oblique incidence, enabled detection of subtle features otherwise impossible to detect in 1D ivdfs, e.g., the anistropy of the fast ion population, ${ }^{12}$ confirmation that metastable quenching is the dominant mechanism for reducing the LIF signal amplitude of the fast ion population in expanding helicon plasmas, and evidence of pitch-angle scattering for the most energetic ions. The shapes of the slow and fast 2D ivdfs were consistent with different origin locations for the two ion populations: the slow population is a locally created background population whereas the fast population is created in the source, undergoes acceleration through the potential drop of the EDL, and has its ivdf broadened by collisions experienced during transit to the measurement location. The symmetrical stretching towards lower absolute speeds of the 2D ivdf proved is inconsistent with upstream perpendicular heating followed by an adiabatic upwelling; the mechanism responsible for the "ion conics" observed in auroral EDLs and in laboratory experiments. 
In summary, this study identified some characteristics of ion acceleration mechanisms in single and multicomponent ion plasma in the expansion region of the helicon plasma. Further experimental investigations and theoretical approaches are needed to distinguish between the different possible ion acceleration mechanisms in the diverging magnetic field region. Among other works dedicated to this phenomenon, I hope this dissertation will be a starting point for such investigations. 


\section{Chapter 8 References}

${ }^{1}$ C. Biloiu, X. Sun, E. Scime, and B. McGeehan, Rev. Sci. Instrum. 75, 4296 (2004)

${ }^{2}$ C. Charles and R. Boswell, Appl. Phys. Lett. 82, 1356 (2003)

${ }^{3}$ A. M. Keesee, PhD Dissertation, West Virginia University (2006)

${ }^{4}$ K. Takahashi, C. Charles, R. W. Boswell, and R. Hatakeyama, Phys. Plasmas 15, 074505 (2008)

${ }^{5}$ X. Sun, S.A. Cohen, E. E. Scime, and M. Miah, Phys. Plasmas 12, 103509 (2005)

${ }^{6}$ C. S. Corr, J. Zenger, R. W. Boswell, and C. Charles, Appl. Phys. Lett. 91, 241501 (2007)

${ }^{7}$ N. Sadeghi, N. Dorval, J. Bonnet, C. Kadlec-Philippe, and A. Bouchoule, $35^{\text {th }}$ AIAA/ASM/SAE/ASEE/ Joint Propulsion Conference \& Exhibit, June 20-23, Los Angeles, CA (1999)

${ }^{8}$ R. J. Cedolin, W. A. Hargus Jr., P. V. Storm, R. K. Hanson, and M. A. Cappelli, Appl. Phys. B 65, 459 (1997)

${ }^{9}$ C. Charles, R. W. Boswell, and M. A. Lieberman, Appl. Phys. Lett. 89, 261503 (2006)

${ }^{10}$ R. Murphy, I. A. Biloiu, M. Spencer, R. Hardin, and E. E. Scime, $47^{\text {th }}$ American Physical Society Division of Plasma Physics Meeting, October 24-28, Denver, CO (2005)

${ }^{11}$ I. A. Biloiu, E.E. Scime, and C. Biloiu, Plasma Sources Sci. Technol., submitted

${ }^{12}$ I. A. Biloiu, E. E. Scime and C. Biloiu, Appl. Phys. Lett. 92, 191502 (2008) 
VITAE

\section{IOANA A. BILOIU}

\section{EDUCATION:}

Doctor of Philosophy in Physics

2009 West Virginia University Morgantown, WV

Master of Science in Optical Techniques and Plasma \& Lasers NonConventional Technologies

1998 University of Bucharest

Bucharest, Romania

Bachelor of Science in Physics

1997 University of Bucharest

Bucharest, Romania

\section{AWARDS \& HonORS:}

Best student presentation award, ICOPS 2008 - The $35^{\text {th }}$ IEEE International Conference on Plasma Science, June 15-19 (2008), Karlsruhe, Germany.

\section{Professional Membership:}

The American Physical Society - Division of Plasma Physics

The Institute of Electric \& Electronic Engineers - Division of Plasma Physics

\section{Publications \& Presentations: Refereed publications}

One- and two- dimensional laser induced fluorescence at oblique incidence

Ioana A. Biloiu, Earl E. Scime, and Costel Biloiu

Plasma Sources Science and Technology, 18 (2009) 025012

Increased upstream ionization due to formation of a double layer

S. Chakraborty Thakur, Z. Harvey, I. A. Biloiu, A. Hansen, R. A. Hardin, W. S. Przybysz, and E. E. Scime

Physical Review Letters, 102 (2009) 035004

Ion beam acceleration in a divergent magnetic field

Ioana A. Biloiu, Earl E. Scime, and Costel Biloiu

Applied Physics Letters, 92 (2008) 191502

Two-dimensional argon-ion velocity distributions in the expansion region of a helicon plasma source Ioana A. Biloiu, Earl E. Scime, Costel Biloiu and Samuel A. Cohen IEEE Transactions on Plasma Science, 36 (2008) 1216

Temporal evolution of bimodal argon-ion velocity distribution in an expanding helicon plasma Ioana A. Biloiu and Earl E. Scime

IEEE Transactions on Plasma Science, 36 (2008) 1376 
Nitrogen dissociation degree in the diffusion region of a helicon plasma source obtained by atomic lines to molecular band intensities ratio

Costel Biloiu, Earl E Scime, Ioana A. Biloiu and Xuan Sun

Journal of Applied Physics, 102 (2007) 053303

High time resolution laser induced fluorescence in pulsed argon plasma

Ioana A. Biloiu, X. Sun, and E. Scime

Review of Scientific Instruments, 77 (2006) 10F301

Amorphous flurocarbon polymer films ( $a-\mathrm{C}: \mathrm{F})$ obtained by plasma enhanced chemical vapor deposition from perfluoro-octane $\left(\mathrm{C}_{8} \mathrm{~F}_{18}\right)$ vapor II: dielectric and insulating properties

C. Biloiu, Ioana A. Biloiu, Y. Sakai, H. Sugawara, and A. Ohta

Journal of Vacuum Science and Technology A, 22 (2004) 1158

Amorphous flurocarbon polymer films ( $a-\mathrm{C}: \mathrm{F})$ obtained by plasma enhanced chemical vapor deposition from perfluoro-octane $\left(\mathrm{C}_{8} \mathrm{~F}_{18}\right)$ vapor I: Deposition, morphology, structural and chemical properties

C. Biloiu, Ioana A. Biloiu, Y. Sakai, Y. Suda, and A. Ohta

Journal of Vacuum Science and Technology A, 22 (2004) 13

Enhancement of nitrogen gas breakdown voltage between coated aluminum electrodes with fluorocarbon polymer film prepared in $\mathrm{C}_{8} \mathrm{~F}_{18}$ vapor plasma

C. Biloiu, Ioana A. Biloiu, Y. Sakai, Y. Suda, and M. Nakajima

Japanese Journal of Applied Physics, Part 2, 42 (2003) L201

The molecular excitations with change of shape

G. Stanescu and Ioana A. Biloiu

Proceedings of SPIE - The International Society for Optical Engineering, 4430 (2001) 697

The measurements of high values refractive index

St. Levai, M. Bazavan, O. Toma, Ioana A. Biloiu

Romanian Reports in Physics, 53 (2001) 79

New model concerning the hollow cathode electric discharge (HCED) as a spectral source in mixed noble gases and metal vapors

I. Iova, M. Bazavan, C. Biloiu, Gh. Ilie, and Ioana A. Biloiu

Proceedings of SPIE - The International Society for Optical Engineering, 4430 (2001) 77-81

On the electrical field dependence of glow discharge parameters

I. Mustata, Ioana A. Biloiu, C. Biloiu, and C. I. Pop

Romanian Journal of Physics, 45 (2000) 561

On the mechanism of the relaxation pulse glow discharge

I. Mustata, C. Biloiu, G. F. Leu, M. Cretu, Ioana A. Baragan, and A. Salabas

Romanian Reports in Physics, 52, (2000) 71

\section{Conference Proceedings}

Bimodal argon ion velocity distribution functions downstream of an expanding plasma helicon source Ioana A. Biloiu, E. E. Scime, C. Biloiu and S. A. Cohen

The XXVIII-th ICPIG, Int'l Conf. on Physics of Ionised Gases, July 15-20 (2007),Prague,Czech Republic

Published in Conference Proceedings, Eds. J. Schmidt, M. Simek, S. Pekarek and V. Prukner, pg. 1537 
Method for dissociation degree determination in nitrogen plasma from atomic lines to molecular band intensities ratio

C. Biloiu, E. E. Scime, I. A. Biloiu, and X. Sun

The XXVIII-th ICPIG, Int'l Conf. on Physics of Ionised Gases, July 15-20 (2007),Prague,Czech Republic

Published in Conference Proceedings, Editors M. Simek, S. Pekarek, and V. Prukner, on CD, pg. 1598

Amorphous Fluorocarbon Polymer (a-C:F) Films Prepared in Perfluorooctane $\left(\mathrm{C}_{8} \mathrm{~F}_{18}\right)$ Vapor RF Plasmas C. Biloiu, I. A. Biloiu, A. Ohta, Y. Sakai, and Y. Suda

The XXVI-th ICPIG, Int'l Conf. on Physics of Ionised Gases, July 15-20 (2003), Greifswald, Germany

Published in Conference Proceedings, Eds. J. Meichsner, D. Loffhagen, and H.-E. Wagner, vol. 3, postdeadline paper, pg. 59

Amorphous Fluorocarbon polymer films prepared in perfluorooctane $\left(\mathrm{C}_{8} \mathrm{~F}_{18}\right)$ vapor plasma CVD for an electrical insulation application

C. Biloiu, I. A. Biloiu, Y. Sakai, Y. Suda, and A. Ohta

ISPC 16, $16^{\text {th }}$ International Symposium on Plasma Chemistry, June 22-27 (2003), Taormina, Italy

Published in Symposium Proceedings, pg. 500

High Speed Deposition of Amorphous Fluorocarbon polymer (a-C:F) Films using perfluorocarbon Vapor RF plasma CVD

C. Biloiu, I. A. Biloiu, Y. Sakai, A. Ohta, and Y. Suda

SPP-20, The 20-th Symposium on Plasma Processing, January 29-31 (2003), Nagaoka, Japan

Published in Symposium Proceedings, Ed. Kouichi Ono-Kyoto University, pg. 285-286

Breakdown Voltage and Secondary Electron Emission Coefficient of Fluorocarbon Polymer Film coated Electrode Deposited in $\mathrm{C}_{8} \mathrm{~F}_{18}$ vapor RF Plasma in Nitrogen Gas

Y. Sakai, C. Biloiu, I. A. Biloiu, and Y. Suda

2002 Joint Conference of Asian Conference on Electrical Discharge \& Korea-Japan Symposium on High Voltage Engineering, November 17-20 (2002), Soongsil University, Seoul, Korea

Published in Conference Proceedings, vol. II, pg. 379-382

Effect of Fluorocarbon Polymer Film Deposition on Electrodes Breakdown Voltage in a $\mathrm{N}_{2}$ Gas

C. Biloiu, I. A. Biloiu, Y. Sakai, Y. Suda, H. Sugawara, M. A. Bratescu, and M. Nakajima

The XXV-th ICPIG, Int'l Conference on Physics of Ionised Gases, July 16-22 (2001), Nagoya, Japan

Published in Conference Proceedings, Editor T. Goto, vol. II, pg.129-130,

\section{Invited Talks}

Fast and slow ion populations in expanding helicon plasma observed by laser induced fluorescence tomography

I. A. Biloiu and E. E. Scime

The 35-th IEEE, International Conference on Plasma Science, June 15-19, 2008, Karlsruhe, Germany

\section{Selected poster presentations}

Effects of light mass doping on ion velocity distribution functions in an electric double layer

Ioana A. Biloiu and Earl E. Scime

The 50-th Ann. Meeting of the Division of Plasma Physics, November 17-21, 2008, Dallas, USA

Abstract in the Bulletin of the American Physical Society, 53, 14, pg. 311 (2008)

Time evolution of fast ions created in an expanding helicon plasma

Ioana A. Biloiu, Earl E. Scime and Costel Biloiu

The 35-th IEEE Intenational Conference on Plasma Science, June 15-19, 2008, Karlsruhe, Germany 
Ion flows in a two-component expanding plasma

Ioana A. Biloiu and Earl. E. Scime

The 49-th Ann. Meeting of the Division of Plasma Physics, November 12-16, 2007, Orlando, USA

Abstract in the Bulletin of the American Physical Society, 52, 16, pg. 344 (2007)

Effect of the magnetic field divergence on the ion velocity distribution in the expanding region of an argon helicon plasma

Ioana A. Biloiu, E. E. Scime, C. Biloiu and S. A. Cohen

The 60-th Gaseous Electronics Conference, October 2-5, 2007, Arlington, Virginia, USA

Abstract in the Bulletin of the American Physical Society, 52, 8, pg. 45 (2007)

High time resolution LIF in pulsed argon plasma

Ioana A. Biloiu and Earl E. Scime

The 48-th Ann. Meeting of the Division of Plasma Physics, Oct. 30-Nov. 03, 2006, Philadelphia, USA

Abstract in the Bulletin of the American Physical Society, 51, 7, pg. 49 (2006)

High time resolution laser induced fluorescence in pulsed argon plasma

Ioana A. Biloiu, X. Sun, and E. Scime

$16^{\text {th }}$ Topical Conference on High Temperature Plasma Diagnostics Conference, May 07-11, 2006, Williamsburg, Virginia, USA 